University Library

Loughborough

University

Author/Filing Title

DUMBUYA

Class Mark

Please note that fines are charged on ALL overdue items.

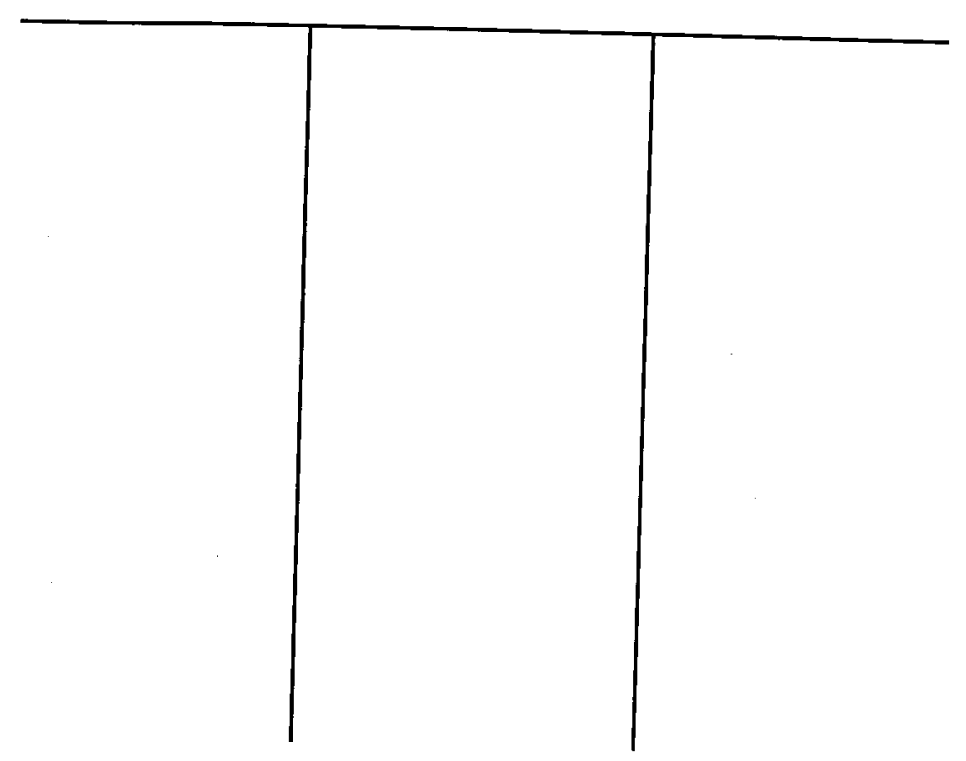





\title{
Visual Perception Modelling for Intelligent Virtual Driver Agents in Synthetic Driving Simulation
}

\author{
By
}

Abdulai Don Dumbuya

A doctoral thesis submitted in partial fulfilment of the requirements for the degree of Doctor of Philosophy

January 2003

Wolfson School of Mechanical and Manufacturing Engineering Loughborough University 


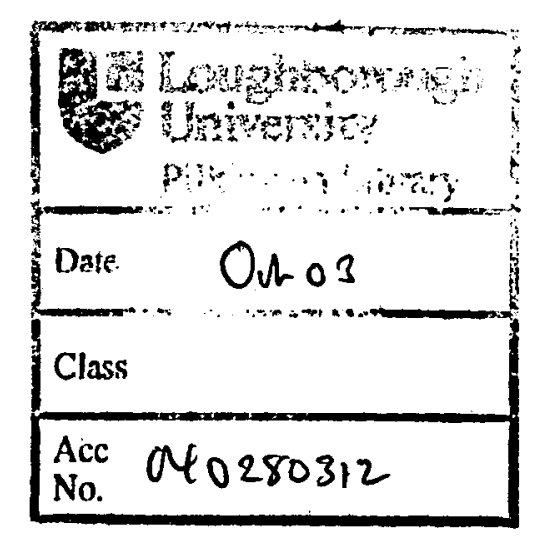




\section{ABSTRACT}

This thesis documents new research into the modelling of driver vision and the integration of a new vision model into a microscopic traffic simulation tool. It is proposed and demonstrated that modelling of driver vision enhances the realism of simulated driver decision-making and behaviour, in turn, leading to improved simulation of driver interactions and traffic flow.

Driving and traffic related research has traditionally fallen into the three distinct areas of driver psychology, traffic and highway engineering and vehicle dynamics, with modelling or experimentation in any of these areas supported by significant approximation in the others. In contrast to this, the vision research discussed here has been carried out in a context that aims to integrate all of these areas equally. This has been realised through the implementation of a new modelling environment, Synthetic Driving SIMulation, SD-SIM. Within SD-SIM, the driver vision model augments a previously developed model for driver decision-making through the visual detection of other vehicles and perception of their behaviour. This removes the gross approximation, found in other contemporary microscopic driving simulation frameworks, of 'perfect vision' where drivers simply share data concerning vehicle positions and speeds.

The novelty of the approach in SD-SIM is that it provides an opportunity to model intelligent virtual drivers agents that are capable of perceiving their own environment, making decisions based on what they 'see' and taking appropriate actions. This allows proactive and reactive interactions between driver agents, reducing the need to be prescriptive in modelling driving scenarios. Furthermore, SD-SIM contributes to the understanding of traffic interactions through the examination of alternative traffic scenarios or "what if" analyses to obtain quantitative estimates of driver behaviour and its consequences. SD-SIM has been validated through comparison with real-life driving experiments in addition to the development of a case study based on real-life accident data. The experimental evaluation measures the performance of virtual driver behaviour and the case study provides functional evaluation to assess scenario modelling and quantitative estimates of driver behaviour. 


\section{Keywords}

Visual perception modelling, Scene En-capturing and Evaluation (SEE), Intelligent Virtual Driver (IVD), driver decision making, Synthetic Driving SIMulation (SDSIM), vision mediated behaviour. 


\section{ACKNOWLEDGEMENT}

I would like to start by expressing my warmest thanks to my supervisor, Robert L. Wood, for sharing his expert and interdisciplinary knowledge, his friendship, patience, and for his continuing support. We have learned together through the numerous discussions we have had but most of all, I have really enjoyed working with and for him. I am grateful to Prof. Chris Backhouse, former head of the department, for supporting my departmental funding for this work, which helped top up my scholarship from my anonymous sponsor.

I would also like to thank all my friends for their friendship, encouragement and support and to Mr. C. Turner and Mr. G. Charles for their technical skills and helping with the testing in some of my experiments. Many thanks to the Leicestershire Constabulary, Traffic Division, especially Chief Inspector Diane Lowe, Inspector Michael Allen, Sergeant Mike McGuinness and PC Sharpe for supporting and providing equipment used in the performance of experiments and collection of driving data. In a similar vain, my acknowledgement goes to the On-The-Spot (OTS) team at Loughborough's Vehicle Safety Research Centre, in particular, Pete Thomas and Julian Hill, for their collaboration and allowing me access to accident data used in the case study. In addition, I would like to express my thanks to all the subjects who took part in the driving experiments.

Finally, I would like to express most gratitude to my parents and families for their unconditional love. Special thanks and loving memories goes to my friends who have adopted me as a member of their families including Mr. and Mrs. Alan and Margaret Atkins and their families, Mr. and Mrs. Brian and Mary Calvert, Mr. and Mrs. Andrew and Sara Brown and their families, Mr. and Mrs. Dietrich and Elke Stechemesser and their families and Mr. and Mrs. Stan and Hilary Cramer. Warmest thanks also go to all the staff of Leonard Cheshire International and Leonard Cheshire Home, Freetown especially Mrs. Lynette Learoyd, Mr. Mark O’Kelly, Mrs, Melrose Cotay, Mr. Rupert Ridge and, for their support and helping to instil a belief in me that there is a future beyond disability. Many thanks (and apologies) also to everybody I have unintentionally missed out of this acknowledgement. 


\section{DEDICATION}

This thesis is dedicated to my anonymous benefactor without whom none of this would have been possible.

To my beloved uncle, Mr. Bassie Bangura who is sadly not here to witness my achievements but I know he is watching over me everyday.

-- Catherall:

The three foundations of learning: Seeing much, Suffering much, and Studying much. To God be all the glory, the honour and thanks for this project! 


\title{
TABLE OF CONTENTS
}

\author{
DECLARATION
}

ABSTRACT

iv

ACKNOWLEDGEMENT

DEDICATION

vii

TABLE OF CONTENTS

viii

\section{CHAPTER 1}

INTRODUCTION

1.0 Introduction 1

1.1 The Research Problem 2

1.2 Research Aims and Objectives 2

1.3 Motivations 3

1.4 Scope of the Research 5

1.4.1 Context of the Research: Framework for Realistic Traffic Simulation 7

1.4.2 Review of Vehicular Traffic Modelling and Simulation 8

1.4.3 Review of Vision and Visual Perception Modelling 8

1.4.4 Image Capturing Processes 9

1.4.5 Computational Visual Information Processing Mechanisms 9

1.4.6 A Synthetic Driving Simulation Framework 9

1.4.7 Implementation of the Synthetic Driving Simulation Framework 10

$\begin{array}{ll}\text { 1.4.8 Validation and Analysis of Vision Mediated behaviour } & 10\end{array}$

$\begin{array}{lll}1.5 & \text { Research Contributions } & 10\end{array}$

$\begin{array}{ll}1.6 & \text { Outline of Thesis Structure } \\ \end{array}$ 
CHAPTER 2

REVIEW OF VEHICULAR TRAFFIC MODELLING AND SIMULATION

2.1 Introduction

2.2 Key Elements of Vehicular Traffic Flow

2.2.1 The Road Environment and Road Network Modelling

2.2.2 The Vehicle and Vehicle Modelling

2.2.3 The Driver and Driver Modelling

2.2.4 Interactions within Traffic Flow

2.3 Modelling Driver Behaviour

2.3.1 Elements of Driver Behaviour

2.3.1.1 Cognitive Models

2.3.1.2 Decision Making Models

2.3.1.3 Adaptive Control Models

2.3.2 Car Following

2.3.3 Lane Changing

2.3.4 Validation of Driver Behaviour Models

2.4 Traffic Generation for Driving Simulation 28

2.4.1 Macroscopic Traffic Simulation 29

2.4.2 Mesoscopic Traffic Simulation 29

2.4.3 Microscopic Traffic Simulation 29

2.4.4 Agent-Oriented Traffic Simulation 31

2.4.5 Synthetic Driving Environments $\quad 32$

2.4.6 Scenario Modelling $\quad 33$

2.5 Chapter Summary 34

\section{CHAPTER 3}

REVIEW OF VISION AND VISUAL PERCEPTION MODELLING 36

3.1 Introduction 36

3.2 A Basis for Biological Vision 36

3.3 Computational Visual Information Processing 39

3.3.1 Bottom-Up Perceptual Information Processing 40

3.3.2 Top-Down Perceptual Information Processing 41

3.4 Synthetic Vision for Autonomous Agents 42

3.5 Visual Perception in Virtual Environments 43

$\begin{array}{lll}3.5 .1 & \text { Boids } & 44\end{array}$

3.5.2 Polyworld 44

$\begin{array}{lll}3.5 .3 & \text { Creatures } & 45\end{array}$ 
3.5.4 Silas T. Dog 46

3.5.5 Artificial Fish 46

3.5.6 Virtual Humans $\quad 47$

3.6 Visual Perception Factors in Driving Simulations 48

3.6.1 Driver Vision Models 49

3.6.2 Distance and Depth Perception $\quad 50$

3.6.3 Speed and Velocity Perception $\quad 51$

3.6.4 Motion Perception $\quad 51$

3.7 Relevant Computational Vision Techniques 53

3.7.1 Computer Graphics $\quad 53$

3.7.2 Machine Vision _... . . 56

$\begin{array}{lll}3.8 & \text { Chapter Summary } & 58\end{array}$

\section{CHAPTER 4}

FUNCTIONAL DESIGN OF SYNTHETIC DRIVING SIMULATION 60

$\begin{array}{lll}4.1 & \text { Introduction } & 60\end{array}$

$\begin{array}{lll}4.2 & \text { Modelling Requirements and Specifications } & 60\end{array}$

4.3 Architecture of the Synthetic Driving Simulation Framework 61

4.3.1 The Intelligent Virtual Driver 63

4.3.1.1 The Visual Perception Model (VPM) 63

4.3.1.2 The Decision Making Model (DMM) 64

4.3.1.3 The Execute Action Model (EAM) 67

4.3.2 The Vehicle Dynamics Model (VDM) 67

4.3.3 The Synthetic Traffic Environment (STE) 67

4.4 Visual Perception Modelling For Virtual Driver Agents 68

4.4.1 Geometric Properties of the Visual Perception Model 69

4.4.2 The Image Capturing Process 71

4.4.3 Computational Visual Information Processing Mechanisms $\quad 76$

4.4.3.1 Image Representation Heuristics 78

4.4.3.2 Image Interpretation Heuristics 84

4.5 An Integrated Simulation Framework 90

4.5.1 System Interactions $\quad 92$

$\begin{array}{lll}\text { 4.5.2 Scenario Modelling } & 96\end{array}$

$\begin{array}{ll}\text { 4.5.3 Animated Visualisation } & 99\end{array}$

$\begin{array}{ll}\text { 4.6 Chapter Summary } & 100\end{array}$ 


\section{CHAPTER 5}

IMPLEMENTATION OF SYNTHETIC DRIVING SIMULATION 102

$\begin{array}{lll}5.1 & \text { Introduction } & 102\end{array}$

5.2 The Graphical User Interface 102

5.2.1 Configuration of People 104

5.2.2 Vehicle Configuration 106

$\begin{array}{ll}\text { 5.2.3 Road Network Configuration } & 107\end{array}$

5.2.4 Qualitative Versus Quantitative Visualisation 107

5.3 Modelling and Visualisation of Intelligent Driver Behaviour $\quad 110$

5.3.1 Binary Image Capture 111

5.3.2 Visual Information Processing 113

5.3.3 Influence of Vision on Modelled Driver Decisions and Traffic Flow 115

5.3.3.1 Example Scenarios $\quad 116$

5.3.3.2 Distance and Speed Perception 119

5.3.3.3 Motion and Looming Detection $\quad 123$

5.3.3.4 Car Following 123

5.3.3.5 Lane Changing $\quad 125$

$\begin{array}{ll}\text { 5.3.4 Execute Action } & 127\end{array}$

$\begin{array}{lll}5.4 & 129\end{array}$

5.4.1 Hardware and Software Considerations 131

$\begin{array}{ll}\text { 5.4.2 System Performance } & 131\end{array}$

$\begin{array}{lll}5.5 & \text { Chapter Summary } & 132\end{array}$

\section{CHAPTER 6}

ALGORITHM PERFORMANCE MEASURES

$\begin{array}{lll}6.1 & \text { Introduction } & 133\end{array}$

6.2 Comparative Studies of Perceptual Factors 134

6.2.1 Optical Flow Information 134

$\begin{array}{ll}\text { 6.2.2 Time To Collision } & 140\end{array}$

6.2.3 Driving Headways 142

6.2.4 Discussion of Comparative Studies 147

6.3 Comparison with Real World Scenarios 148

$\begin{array}{lll}\text { 6.3.1 Data Collection } & 148\end{array}$

6.3.1.1 General Method $\quad 148$

6.3.1.2 Data Sets 153

6.3.2 Validation of Scene En-capturing Evaluation (SEE) 154

6.3.2.1 Calibration of Scene En-capturing Evaluation (SEE) 154 
6.3.2.2 Distance Estimation $\quad 155$

6.3.2.3 Speed Estimation $\quad 158$

6.3.2.4 Eye Resolution and Computational Considerations $\quad 161$

6.3.3 Validation of Intelligent Virtual Driver Behaviour 165

6.3.3.1 Lane Changing 166

6.3.4 Discussion of Real World Scenarios 168

6.4 Case Study: Real-Life Scenario Modelling 170

$\begin{array}{ll}\text { 6.4.1 The Accident Scenario } & 170\end{array}$

6.4.2 Parametric Modelling Procedure 172

6.4.3 Analysis of Road Accident Data 174

$\begin{array}{lll}\text { 6.5 Chapter Summary } & 176\end{array}$

\section{CHAPTER 7}

CONCLUSIONS AND FUTURE RESEARCH 177

$\begin{array}{lll}7.1 & \text { Introduction } & 177\end{array}$

$\begin{array}{ll}7.2 & \text { Driver Vision and Visual Perception } \\ & 177\end{array}$

7.3 Autonomous Driver Agents and Emergent Traffic Flow 177

7.4 Conclusions from the Research 178

$\begin{array}{lll}7.5 & \text { Recommendations for Further Work } & 179\end{array}$

7.5.1 Advanced Vehicle Dynamics Model and Driver Actions 179

7.5.2 Realistic Road Networks and Traffic Environment 181

7.5.3 Enhancement of Vision Model using Animat Vision 181

7.5.4 Improving Driver Decision Making 182

7.5.5 A Brain Model for Virtual Driver Agents 183

7.5.6 Further Validation Using Motorway Driving Experiments 183

$\begin{array}{lll}7.6 & \text { Potential Applications of SD-SIM } & 184\end{array}$

$\begin{array}{ll}\text { REFERENCES } & 186\end{array}$

\section{APPENDIX A}

$\begin{array}{ll}\text { LIST OF PUBLICATIONS } & 204\end{array}$

\section{APPENDIX B}

SELECTED UNIFIED MODELLING LANGUAGE (UML) DIAGRAMS 206

B.1 Attributes and Methods of Key Classes of Synthetic Driving Simulation (SD-SIM) 206

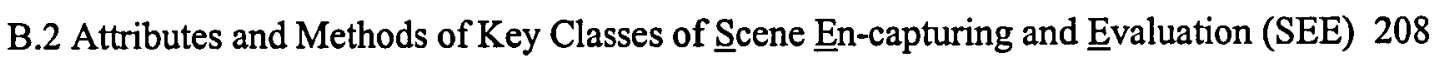


APPENDIX C

DATA COLLECTION TOOLS

210

C.1 A Questionnaire to Asses Subjects Driving Behaviour 210

C.2 General Instructions 211

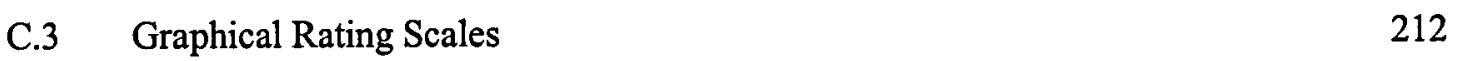




\section{CHAPTER 1}

\section{INTRODUCTION}

\subsection{Introduction}

The behaviour of traffic participants strongly depends on what they see and what they choose to do with the information gathered through seeing. Therefore, visual perception modelling is an important aspect of the virtual driver agents within traffic simulation frameworks. Although vision and visual information processing is complex, at the same time, various models of visual perception have been developed and applied in the areas of Artificial Life (ALife), Artificial Intelligence (AI) and Agent Based Simulation (ABS) to help facilitate complex autonomous behaviour. In these systems, vision is an important part of the interaction between agents, for example, Boids (Reynolds 1987), Polyworld (Yaeger 1994), Virtual Actors (Thalmann 1995), Silas T. Dog (Blumberg 1997), Creatures (Grand et al 1997) and Fish (Tu 1999). Many of these virtual environments have been developed to explore biology and evolution type behaviour, typically using simple sets of logical rules or other computational techniques to generate wide ranging emergent phenomena. It is therefore possible to adopt some of the vision paradigms reported in ALife, AI and ABS systems to enhance autonomous virtual driver agent behaviour within traffic/driving simulations.

The use of driving simulators and traffic simulation models ${ }^{1}$ is becoming increasingly popular. These simulation models provide safe, controlled and cost effective prediction tools in many applications, such as the analysis of traffic flow, highway design, driver psychology, human driver performance within virtual environments and road accident investigations. There are however, different approaches to modelling different levels of detail within these models. Microscopic simulation models offer significant opportunities for representing the complexity of traffic flow that emerges from individual driver behaviour and vehicle interactions. At the same time, the application of Artificial Intelligent (AI), Artificial Life (Alife) and Agent concepts

\footnotetext{
${ }^{1}$ Traffic simulation models tend to refer to the software architecture of the driving simulator whilst driving simulators generally refer to the combination of a traffic generation module and fixed or motion base vehicle cabin.
} 
within microscopic traffic simulation offer the opportunity to simulate realistic behaviour through efficient model abstraction whilst minimising software complexity. Furthermore, visual perception modelling for an ALife system is relatively simple, and is used to support the emergence of complex behaviour within the overall system being modelled. Therefore, the same can be expected through the introduction of vision modelling in driving simulation, providing new opportunities to investigate and mimic vision mediated cognitive processes such as object detection and recognition, collision avoidance, speed and distance perception within virtual driver agents.

\subsection{The Research Problem}

Explicit access to the actual positions and velocities of other driver agents in the simulation is the most unrealistic approximation of driver vision in many contemporary driving simulation frameworks. This amounts to each driver having 'perfect global vision'. Clearly, this is not realistic since human estimation of other vehicles' position, speed and direction depends on subjective interpretation of changes in the driver's local visual environment. As a consequence, a visual perception model is required to remove unrealistic perfect global vision, intending to enhance the realism of simulated driver decision making and behaviour.

\subsection{Research Aims and Objectives}

The primary aim of the research presented in this thesis is to investigate driver vision and visual information processing leading to the modelling of these capabilities for virtual driver agents in synthetic driving simulations. By introducing the new vision model a further aim is to make a significant contribution to the realism of driver decision making and behaviour. Consequently, the research has the following objectives:

(i) To study simple physiological capabilities of biological vision systems such as humans, with a view to identifying distinct functional aspects that can support the structure and mechanisms of the vision model. 
(ii) To critically review, contemporary driving/traffic simulation and vision/visual perception modelling. This will identify the need for visual perception modelling to support visual navigation and vision driven behaviour within autonomous driver agents operating in virtual environments.

(iii) To develop a novel method of synthetic vision that is capable of detecting the presence of objects as well as enabling the creation of cognitive models for visual information processing.

(iv) To develop a realistic driving simulation framework in which intelligent driver behaviour depends on perception-decision-action processes. This framework should exploit concepts used in Artificial Life, Artificial Intelligence and Agent technology to represent the inherent unpredictability and autonomous behaviour of drivers within traffic simulation models. Furthermore, this framework should explicitly integrate the three components of driving i.e. the driver, the vehicle and the traffic environment.

(v) To realise the framework using object oriented techniques and existing programming paradigms e.g. $\mathrm{C} / \mathrm{C}++$ language as well as provide validation of the results against real life driving experiments. In addition to comparison of the results, real-life scenario modelling should be demonstrated.

\subsection{Motivations}

The research reported here has been motivated by the following factors: Firstly to remove the unrealistic availability of 'perfect knowledge' concerning the positions and velocities of vehicles in driving simulations through the introduction of a filtering process, based on the essential elements of vision used by drivers, which is inherently uncertain. Secondly, to do this using sound engineering principles so that the vision model can be rigorously validated and compared with real world experience. Thirdly, to generate essential quantitative vision data that can be built upon either quantitatively or qualitatively through subsequent use of various cognitive models. Fourthly, to develop a realistic traffic simulation environment in which driver behaviour, traffic generation and vehicle dynamics can have equal focus. Finally the 
research reported here has been motivated by work in the four main areas; Artificial Life, Artificial Intelligence, Computer Graphics and Agent Based Simulation. These concepts offer the opportunity to simulate realistic behaviour.

Artificial Life (ALife), the focus in ALife is on the synthesis of lifelike systems that exhibit behaviour characteristic of natural living systems (Langton 1989). The underlying principle of ALife is the simulation of simple interactions that produce complex emergent behaviour. These elements provide a suitable context in which to describe intelligent behaviour. For example, a range of concepts and metaphors from Artificial Life (ALife) have been adopted in this thesis within the formulation of a visual perception model called SEE (Scene En-capturing and Evaluation). This is based on geometric properties of vision, and visual information processing using simple cognitive assumptions of biological vision systems.

Artificial Intelligence (AI) the definition of AI covers a broad spectrum, however, in (Albus 1991) the author suggests that at a minimum level, intelligence requires the ability to sense the environment, to make decisions and to control action. In this thesis AI supports the concepts of perception-decision-action as well as many of the algorithms developed for the intelligent virtual driver agents.

Computer Graphics (CG) provides techniques suitable for the simulation of vision and behavioural related problems. Computer graphics techniques such as ray tracing enable autonomous agents in virtual environments to render the scene from their view point. For example in (Renault et al 1990, Noser et al 1995, Thalmann 1995, Blumberg 1997, and Bordeux et al 1999) computer graphics provides the ability to build synthetic vision models to acquire information from the environment and support realistic behaviour among the agents. The field of computer graphics and other related fields such as machine vision have motivated the adoption of techniques used in the development of SEE.

Agent Based Simulation (ABS), refers to the representation of 'simulated entities' as agents. In $\mathrm{ABS}$, individual characteristic and behaviour of the entities are explicitly modelled. The agents are referred to as 'intelligent agents' if they are capable of perceiving their environment, learning from the environment, planning to achieve 
their goals and take appropriate actions (Hayes-Roth 1995). In expanding this definition, several attributes can be associated with an intelligent agent e.g. autonomy, reactivity, pro-activeness, mobility, etc. (Wooldridge and Jennings 1995).

There are different types of agents and according to (Franklin and Graesser 1997), they can be categorised into biological, robotics, computational. Computational agents can be further decomposed into software and artificial life agents. Software agents include task specific, entertainment and viruses. This thesis mainly deals with Artificial Life (ALife) Agents, which the thesis author has referred to as 'Virtual Driver Agents'. Furthermore, the relevance of intelligent agent concepts in this thesis is that within the context of software engineering in general, agent-based abstractions are often very similar to traditional object-oriented design methodologies, providing suitable abstraction mechanisms.

\subsection{Scope of the Research}

The scope of the research discussed in this thesis outlines the context of the research undertaken as well as the definition of the specific requirements or tasks to meet the previously stated aims and objectives. These tasks are outlined in the individual chapters of the thesis structure given in figure 1.1 and briefly described in the subsections of 1.4. The roman numerals in figure 1.1 are mapped to these sub-sections. A brief description of each chapter is given in section 1.6.

With regard to figure 1.1, the Synthetic Driving Simulation (SD-SIM) and the incorporated vision model, Scene En-capturing and Evaluation (SEE) described in chapters 4 and 5 (figure 1.1, III and IV) are both outputs from this research. SD-SIM also incorporates a previously developed driver decision making model (Wood and Arnold 1997). 


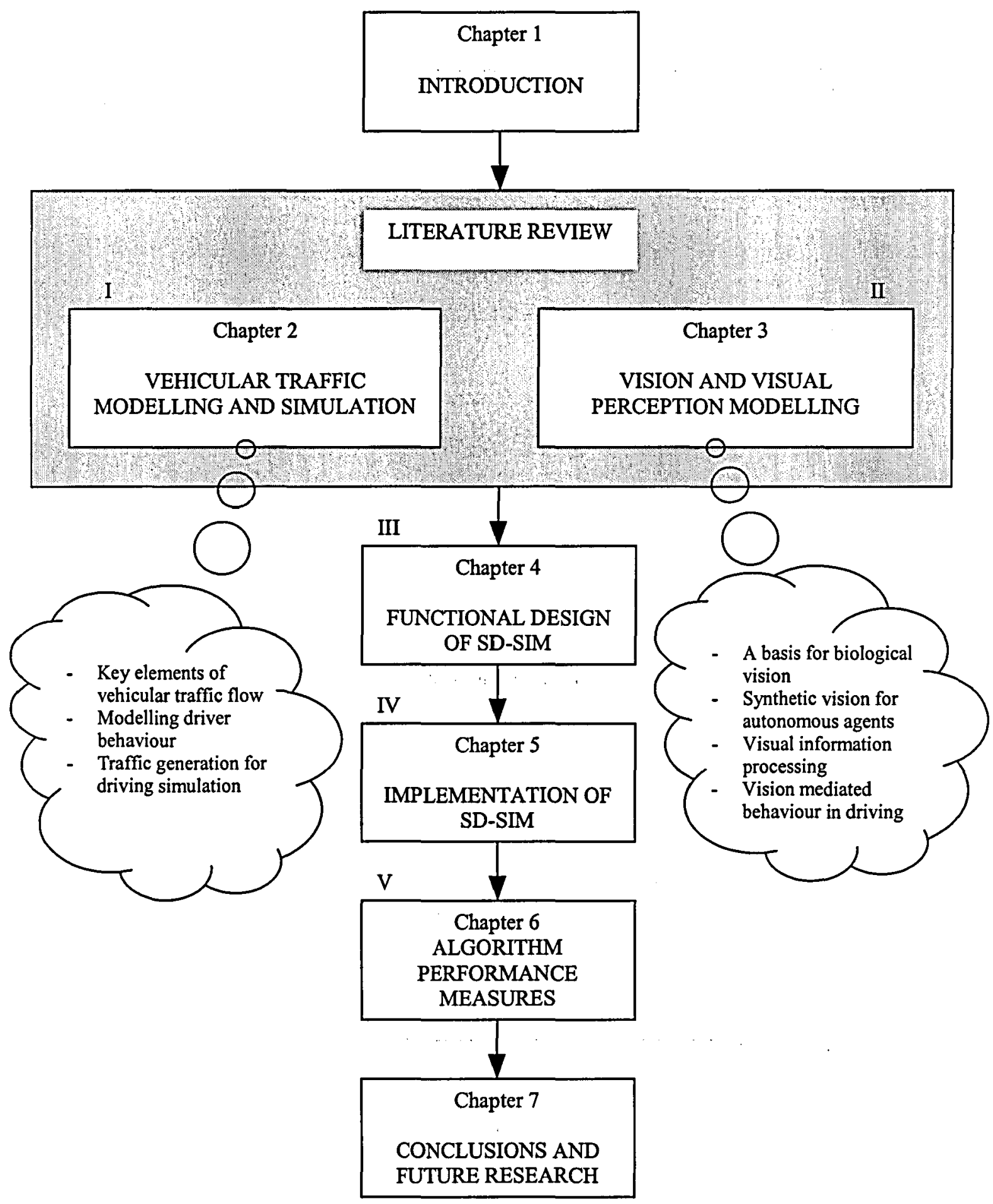

Figure 1.1: The structure of the thesis outline 


\subsubsection{Context of the Research: Framework for Realistic Traffic Simulation}

The requirements for realistic traffic simulation generally depend on the purpose or application of the simulator. Microscopic driving simulation software is generally used in combination with a fixed-base vehicle cabin for safe and controlled experimentation. If the results obtained from such simulators are to be believed, then it is imperative that the different components (driver behaviour, vehicle models and virtual environment) produce realistic and convincing driver and traffic behaviour. Wright $(2000 \mathrm{~b})$ identified three requirements for 'realistic traffic simulation' as intelligence, unpredictability and virtual personality. Intelligence provides the ability for individual vehicles to autonomously respond in 'naturalistic behaviours' with virtual drivers exhibiting real driving styles. This could include vehicle dynamics models, accounting for suspension, braking and cornering forces, etc., and the transfer of these forces to the driver. Unpredictability concerns the addition of uncertainty in the simulation, meaning every driver behaves in a different way. This research proposes that such unpredictability can be modelled through the integration of processes representing driver visual perception capabilities, decision-making, and execution of actions. Virtual personality should address driver characteristics in the form of aggressiveness, gender, age, fatigue etc. and the effect of these on their driving behaviour. This thesis proposes that such personalities can either be prescribed or can be emergent results of the lower level modelling of brain function and other bio-chemical factors, for example (Grand et al 1997).

The interactions of the three main components of driving i.e. the driver, vehicle and the environment can be viewed as a typical man-machine system, figure.1.2. Human interaction activities involve perception of the road environment, decision making based on the traffic information and execution of actions through vehicle control. This perception-decision-action sequence is important and should be modelled realistically. 


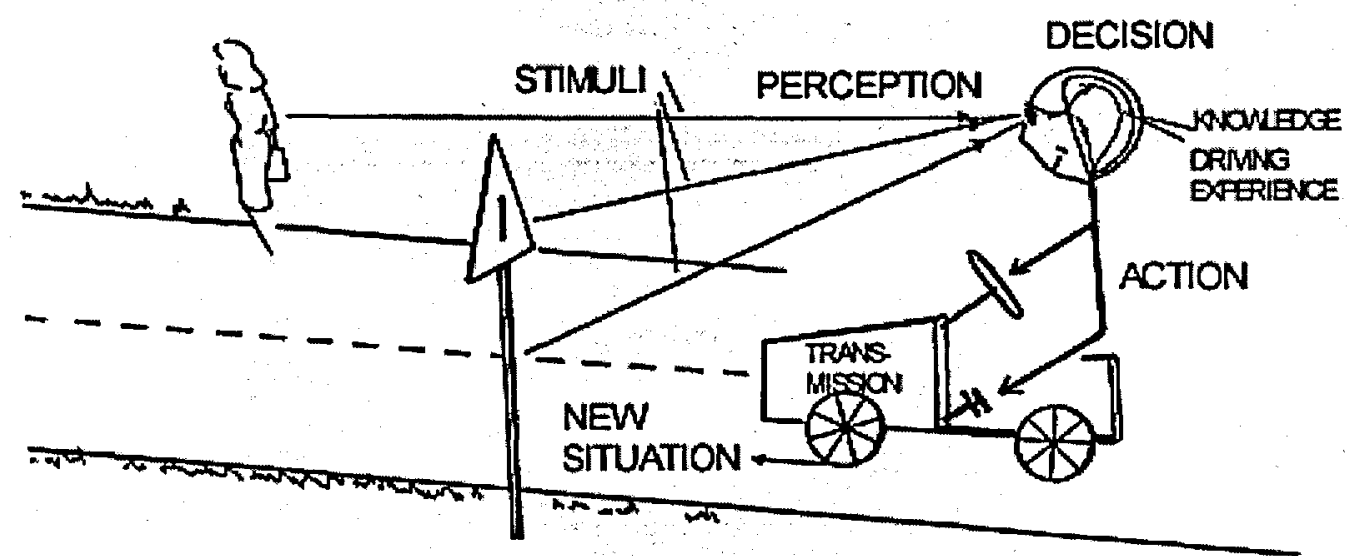

Figure 1.2 Basic components of driving (Cited from Pursula 1999)

\subsubsection{Review of Vehicular Traffic Modelling and Simulation}

The aim of the literature survey is to provide a comprehensive and critical review of the literature covering the two main areas of interest in this thesis. This will be split into two chapters to reflect their broad context. Knowledge of traffic flow characteristics and related techniques is essential in the design, implementation and discussion of traffic simulation models. Vehicular traffic modelling and simulation review (figure 1.1, $\mathrm{l}$ ) focuses on three main themes (1) the key elements of vehicular traffic flow in terms of fundamental traffic flow theory, (2) driver behaviour modelling and (3) traffic generation for driving simulations.

\subsubsection{Review of Vision and Visual Perception Modelling}

The review of vision and visual perception modelling (figure 1.1, II) will revisit various aspects of driver behaviour introduced in section 1.4.2, but from a driver vision perspective. Secondly, the chapter will present an overview of existing vision models within software environments, additionally considering approaches, techniques and tools used to support the development of vision models. In so doing, it is intended to highlight the gap between synthetic vision models and driver visual capabilities. 


\subsubsection{Image Capturing Processes}

The study of image representation in biological system from the literature review will provide basic understanding of the vision process (figure 1.1, II). Based on this understanding, simple assumptions concerning biological vision are used to construct an image capturing process via two artificial eyes. The development of a Scene Encapturing and Evaluation - SEE model will be proposed as part of the Visual Perception Model (VPM) of SD-SIM. The development of the VPM is based on the abstraction of key visual processes into a logical vision model. The construction of SEE is based on the geometrical properties of vision and will provide both image capture and visual information processing. Using the vision model structure, image capturing uses ray tracing techniques so that images of objects within the synthetic driving environment are captured from each driver agent's viewpoint.

\subsubsection{Computational Visual Information Processing Mechanisms}

This work will focus on the development of a computational framework for visual information processing (this also draws from the output of the literature review in figure 1.1, II). Binary images captured at successive times, from the image capturing process are mapped onto a 16-plane 'visual memory' for further processing. Visual information processing heuristics for image representation and interpretation will be formulated. The development of these heuristics will be influenced by cognitive modelling. These heuristics will be responsible for image identification, stereo/mono processing, image tracking, perception of distance and speed, and motion perception through processing binary images obtained from the image capturing process.

\subsubsection{A Synthetic Driving Simulation Framework}

The aim is to develop a conceptual architecture of a synthetic driving simulation framework (figure 1.1, III). The framework will consist of three main components: Synthetic Traffic Environment (STE), Vehicle Dynamics Model (VDM) and Intelligent Virtual Driver (IVD). In this thesis, the STE and VDM are developed only to a minimum level of functionality, with the IVD being the main focus of this work. The IVD module itself will be described in terms of its three main subsystems, the Visual Perception Model (VPM), the Decision Making Model (DMM), and the 
Execute Action Model (EAM). The framework also attempts to replace some of the prescriptive nature of driving simulation models by allowing behaviour to emerge as a consequence of individual agent interactions.

\subsubsection{Implementation of the Synthetic Driving Simulation Framework}

This work will involve the realisation of the synthetic driving simulation framework (figure 1.1, IV). This will provide a new microscopic simulation tool which will allow the user to define scenarios and provides a suite of facilities to support qualitative and quantitative visualisation of traffic flow interactions. Also, implementation will consider the integration of a Virtual Reality Modelling Language (VRML) tool for visualising calculated behavioural data (e.g. distance and speed perception, traffic flow etc.).

\subsubsection{Validation and Analysis of Vision Mediated behaviour}

The aim is to validate the current functionality of some of the features of the developed framework (figure 1.1, V). One aspect that requires validation is the vision model both in its image capturing and visual information processing. Experiments have been conducted with the help of Leicestershire Constabulary, Traffic Division in the UK. In these experiments, subjects' visual perception of positions and speeds of moving vehicles will be measured and compared with estimations from SD-SIM. The quantitative performance analysis of SD-SIM should demonstrate the feasibility of modelling driver vision and its implication to vision mediated behaviour in driving.

\subsection{Research Contributions}

This thesis contributes to the area of Artificial Life, Artificial Intelligence, Agent Based Simulation and Driver Psychology. It combines the underlying principle of ALife in the simulation of simple interactions that produce complex emergent behaviour; endows intelligence to virtual driver agents by allowing them to perceive, make decision and act. It also applies agent-based abstractions in the form of object oriented design methodologies and demonstrates the use of a new computational tool to study driving psychology related issues such as vision mediated behaviour. 
Specifically, the main contributions of the research undertaken and reported in this thesis are:

- The introduction of a new synthetic vision model to remove the unrealistic availability of perfect global vision of virtual driver agents in driving/traffic simulation. This is novel compared to many contemporary driving/traffic simulation frameworks and as such the work reported here has been published in the relevant literature e.g. (Dumbuya and Wood 2003, Dumbuya and Wood (in press)).

- The development of a computational framework for visual information processing that enabled the introduction of deeper cognitive models of driver vision and visual perception. This work was presented in the European Conference on Mathematical Psychology as well as at an invited Seminar at Oxford University (Dumbuya et al 2002a, Dumbuya and Wood 2002c).

- The development of an agent-based traffic simulation framework that integrates driver, vehicle and traffic environment and used to model inherent unpredictability and autonomous behaviour of drivers through scenario modelling. This work has been published in the relevant driving simulation literature (Dumbuya et al 2002b).

- The design and conduction of driving experiments to assess human drivers' ability to estimate distance and speed and the implication of this on road safety was presented in the Association of Industrial Road Safety Officers Meeting (Dumbuya and Wood 2001).

- The structure and functionality of the developed integrated driving simulation framework has been validated against real-life driving data and in particular the vision validation results have demonstrated driver vision modelling within an agent based traffic simulation. A case study application of SD-SIM ability to model accident data was demonstrated in (Wood et al 2003). 
This research also makes a number of technical contributions. These include (i) the adaptation of machine vision algorithms for region labelling and object tracking (Jain et al 1995) and the formulation of new algorithms for image formation and visual information processing integrated into the synthetic vision model. (ii) The development of a new microscopic simulation tool that enables the analysis of psychological factors that influence driving and traffic flow. (iii) The development of a graphical user interface that allows easy specification of driving scenarios, control of visual parameters and visual observations/animations of the effect of specified parameters.

\subsection{Outline of Thesis Structure}

The overall structure of this thesis was shown in figure 1.1. The thesis is organised in seven chapters. Since the work that has been carried out covers broad areas of driving and vision, chapters 2 and 3 provide a critical review on vehicular traffic modelling and simulation as well as vision and visual perception modelling.

Chapter 4 describes the functional design of the Synthetic Driving Simulation SDSIM, discussing the three major components and their subsystems. The main theme of this chapter is the design and development of the vision model functionality which forms a central part of the visual perception model of the intelligent virtual driver.

Chapter 5 discusses the implementation of the functional design of chapter 4 , with particular emphasis on the graphical user interface. This chapter also discusses quantitative and qualitative visualisation of simulation results and demonstrates the process of configuring different driving scenarios within the tool. Some example results from SD-SIM are also presented.

Chapter 6 provides a validation of the current functionality of SD-SIM through comparison of results with real-life driving data. Performance of the tool is discussed in terms of measured data and observational inferences. Both comparative studies and experimental data are used to validate the work. Furthermore, the potential applicability of the tool is demonstrated in this chapter with an accident case study. 
Chapter 7 presents the conclusions drawn from the work along with further research work that could be undertaken. 


\section{CHAPTER 2}

\section{REVIEW OF VEHICULAR TRAFFIC MODELLING AND SIMULATION}

\section{$2.1 \quad$ Introduction}

This chapter presents a broad overview of relevant work in order to provide a clear context for understanding vision research in driving. Knowledge of traffic flow characteristics and related techniques is essential in the design, implementation and discussion of traffic simulation models. The review focuses on three main themes (1) the key elements of vehicular traffic flow and fundamental traffic flow theory, (2) driver behaviour modelling and (3) traffic generation for driving simulations. The chapter is concluded with a summary of the main issues raised to identify the relevant concepts and techniques that form the theory of this thesis.

\subsection{Key Elements of Vehicular Traffic Flow}

The field of traffic flow is diverse and in the last 40 years, traffic flow theories have given significant insights into traffic behaviour and its characteristics. These theories are concerned with understanding interactions between traffic variables and processes through their mathematical representation (Hall 1997). Examples of these variables and processes include flow, speed, traffic density, distance headway, car following, shock wave phenomena, intersection gap acceptance and hydrodynamic analogies. However, the three main elements of vehicular traffic flows are the driver, the vehicle and the road environment (Taylor et al 1996). According to Taylor, each of these elements can be seen as an independent entity with the processes describing the flow as random. This randomness originates from individual driver decisions and actions.

Interactions between the different traffic elements occur on two levels - interrupted and uninterrupted traffic flow (Taylor et al 1996). Interrupted traffic flow concerns the influence of external factors on the traffic stream such as pedestrians, buses and signalled crossings. On the other hand, uninterrupted traffic flow describes traffic streams that are not influenced by external factors but instead by interactions between 
individual vehicles, and between vehicles and the road. Typical examples of uninterrupted traffic flow are flows on highways and motorways. This distinction between the two types of traffic interactions provides a framework in which to identify appropriate models of traffic.

The current focus of the work reported here is based on minimal external influence (e.g. pedestrian and traffic lights) on the traffic flow system and therefore fits between the two descriptions. The fundamental variables in uninterrupted traffic flow are flow rate, traffic density and average vehicle speed, related by equation 2.1 :

$$
q=k \times v
$$

Where, $q$ is the flow rate (volume), defined as the number of vehicles passing a fixed point in a given time period. Flow rate is typically expressed in terms of vehicles per day (veh/day), vehicles per hour (veh/h) or vehicles per second (veh/s).

$k$ is the traffic density (concentration), defined as the number of vehicles occupying a unit length of lane or roadway at a given instant of time. Typical density unit is veh/ $/ \mathrm{km}$.

$v$ is the average speed (velocity) defined as the distance travelled per unit time. Units include $\mathrm{km} / \mathrm{h}$ and $\mathrm{m} / \mathrm{s}$.

\subsubsection{The Road Environment and Road Network Modelling}

The analysis of the road environment is primarily concerned with functional, geometric and structural designs (Drew 1968). Functional design addresses traffic demands and travel, which have an impact on environmental, social and human factors. Geometric road design considers the physical features of the roadway such as lanes, gradients, curvatures, junctions and road furniture. The geometrical design of the roadway affects efficient traffic operations based on driver behaviour and vehicle capabilities within the road environment. In terms of safe vehicle operation, it is intuitive that roads with a variety of bends, corners and junctions often present major hazards. Finally, in structural design the effect of traffic loading is critical to the 
performance conditions on the road. For example road deformation and width of the lane is directly influenced by vehicle weight and width respectively. Thus structural design provides a framework for the assessment of structural integrity and performance predictions in terms of changes in the road and vehicle variables.

Various road and network analysis models exist. In traffic engineering and transportation management systems, road network based traffic flow modelling plays an important part in evaluating traffic congestion, network optimisation, travel demand forecasting and improvements in environment and public transport systems (Smith et al 1996, Maher 1998, Wright and Roberg-Orenstein 1998). Traffic network models are widely applied in the four-stage systems i.e. trip generation, trip distribution, mode split and route assignment. For example, route assignment methodologies such as dynamic traffic assignment (DTA) and stochastic user equilibrium (SUE) describe traffic flow through network links and are essential for assessing network performance. Several computational network models in the fourstage systems have been proposed (see Chatterjee et al 1999, Pursula 1999 for a detailed review). These models are used in analysing travellers' responses, estimating origin-destination (O-D) flows and evaluation of general network and traffic problems. Also, advances in technology such as Driver Information Systems (DIS), e.g. guidance systems, route planning systems and telematics are providing improved network models and analytical tools (Chatterjee et al 1999).

On the other hand, road network modelling for driving simulation tends to focus on the features of the road that can be represented within a database (e.g. Artz 1995, Sabeur and Rommelfanger 2000, Charles and Espié 2000). The construction of these models is usually influenced by highway design and other civil engineering principles and involves the definition of geometric and logical information. Geometric information includes computer imagery produced from an image generator as part of the visual database. Logical information (or scenario data) contains information about lanes, lane type, direction of travel and traffic rules. The construction of road networks based on geometry information is demonstrated in (Artz 1995, Charles and Espié 2000). In (Artz 1995), the road network is modelled as connected segments with geometrical features such as straight, curve, transition spiral and intersection. This network model and other information derived from a visual database form part of 
the computer generated terrain in the Ford Motor Company driving simulator. In (Charles and Espié 2000), the authors adopted a multi-level descriptive elements approach to model geometric road profiles and different road components (e.g. hedges, trees, road signs). The approach describes geometric primitives based on the information they contain. For example, a road or road component has a face, material and texture details. This information is extracted as photometric data to perform rendering and hence visualisation.

In addition to the geometry of the road, logical information can also be specified as part of the road network database. For example, a Logical Road Network (LRN) design is implemented as part of a suite of software in the Leeds Driving Simulator (Bailey 2000). The approach uses rules to decompose road networks into junctions and connecting roads. The LRN utilises logical connectivity information to create road networks and support traffic flow. In (Papelis and Bahauddin 1995), a Virtual Roadway Environment Database (VRED) is described. This database provides logical road information in the form of client/server architecture so that the representation of roads is separated from the behaviour modelling or scenario control. AutoRoad ${ }^{\circledR}$ (Sabeur and Rommelfanger 2000) and EVARISTE RESEAU ${ }^{\circledR}$ (Charles and Espié 2000) are commercial road generation applications capable of producing realistic road features as well as logical road information.

\subsubsection{The Vehicle and Vehicle Modelling}

There is considerable research in automotive (vehicle) engineering (Wong 2001). However this section takes a fundamental view. The mechanics/physics of the vehicle element are often characterised by its combined static, kinematic and dynamic properties. Examples of static characteristics include mass, length, width, height and type. Vehicle kinematics deals with describing the motions of the vehicle in terms of its position, velocity and acceleration/deceleration. A vehicle's kinematic motion is usually described using standard Newtonian equations of motion.

In addition, kinematic analysis is a prerequisite to the development of dynamics analysis. Vehicle dynamics addresses the forces acting on the vehicle that give rise to its motion. Typical forces include friction, rolling and grade resistances. These forces 
affect the kinematic vehicle properties in terms of acceleration, braking, turning (steering), control and stability performance. In addition to these forces, dynamic vehicle behaviour is also influenced by variables such as suspension settings, road geometry and powertrain.

In traffic simulation, vehicle modelling is typically rudimentary, requiring a limited number of parameters. Vehicle models are often represented by simple mathematical or physical properties e.g. kinematic motion and dimension. The reason for such simplistic representation is because these models are usually required to perform relatively simply operations such as planar two-dimensional motions, since much of the emphasis is on driver behaviour (e.g. Druitt 1998). In more advanced vehicle simulation models, vehicle dynamics is incorporated as part of an integrated simulation environment, for example in computer games (Gordon et al 2002). An example of a PC-based advanced vehicle dynamics model VDANL (Vehicle Dynamics Analysis, Non-Linear) was developed through collaboration between Systems Technology Incorporated (STI) and the National Highway Traffic Safety Administration (NHTSA) (Allen et al 1998). This model was developed for the analysis of passenger cars, trucks and articulated vehicles. Its recent improvements, discussed in (Allen et al 2000), include for example steering/speed control, tyres/wheels, brakes, powertrain and driver feedback features. The model architecture has been extensively validated and adopted in many advanced driving simulation environments, e.g. National Advanced Driving Simulator (NADS) (Chrstos and Heydinger 1997), Interactive Highway Safety Design Model (IHSDM) (Paniati and True 1996), CarSim (Sayers 1998) and STISIM (Allen et al 1998).

\subsubsection{The Driver and Driver Modelling}

The driver component of the traffic system is the most complex. This is because the human drivers are generally characterised by higher-level processes such as cognitive functions (learning, motivation and attitude), control functions (psychomotor factors) and perceptual capabilities (vision, hearing and sound). Drew (1968) described in detail some of these processes. Learning concerns the ability of the driver to improve their understanding of the driving task through repetition, whilst feedback allows error adjustment to be made to improve situational responses. Motivational factors affect 
decision-making. For example lower motivation through lack of sleep and fatigue can lead to poor judgement and thus result in fatal accidents. Control functions address the execution of actions, typically those involved with stabilising the vehicle's path and speed e.g. steering, braking and acceleration.

Of the human's many perceptual capabilities vision is the most important of all the senses for driving. Drew commented that, "Many traffic operational and design problems require a knowledge of the general characteristics of human vision" (Drew 1968, p. 28). He further suggested that the most important factors of vision required in driving include object detection, visual acuity, peripheral vision, glare recovery, colour perception and depth (motion) perception. As vision is part of the main theme of this thesis, a thorough review of vision and visual perception modelling is presented in chapter 3 .

Driver behaviour modelling is an important aspect of understanding the driver within the traffic system as well as analysing traffic situations. However, there is a recognised lack of driver behaviour models in the literature. Instead, many aspects of driver behaviour modelling tend to focus on assumptions, theories and techniques required to describe behaviour (Michon 1985). Perhaps this limitation, as already mentioned could be largely due to the fact that the description and representation of driver behaviour is inherently complex. Some authors however have attempted to model this complexity by using a combination of techniques and assumptions such as probability distributions and rule-based systems (Wright 2000a); situational factors and driver characteristics (Sukthankaar 1997); mental state description of cognitive behaviour (Rensink and Boer 1996). A comprehensive review of the methods and techniques employed in driver behaviour modelling is presented in sections 2.3 and 2.4 .

\subsubsection{Interactions within Traffic Flow}

The interactions between the key components of vehicular traffic with respect to time and space can be viewed as a typical human-machine system i.e. driver (human), car (machine) and environment. The driver-vehicle interaction tasks are summarised in (Allen et al 1996, p. 27-28), including: “Control concerns psychomotor functions that 
stabilise vehicle path and speed against various aerodynamic and road disturbances. Guidance involves perceptual and psychomotor functions coordinated to follow delineated pathways, adhere to implied speed profiles, interact with traffic and avoid hazards. Navigation involves higher level cognitive functions applied to path and route selection and decisions regarding higher-level traffic interactions (e.g. avoiding congestion)".

From a control system point of view, the fundamental interaction activities can be described as either open loop or closed loop. The open loop operation is represented as a perception-decision-action sequence. Figure 2.1 illustrates the open loop sequence. This loop involves the human driver receiving information from the road environment, making decisions based on the traffic information and then executing actions to respond accordingly. Closed loop operations on the other hand involve a feedback mechanism that allows actions to be compared to the system input (for example the driver performing mental evaluation of the traffic situation) to measure deviations from the desired goals and institute corrective actions. The open and closed loop sequences are important functionalities in traffic flow interactions and as such should be modelled with some degree of accuracy and realism.

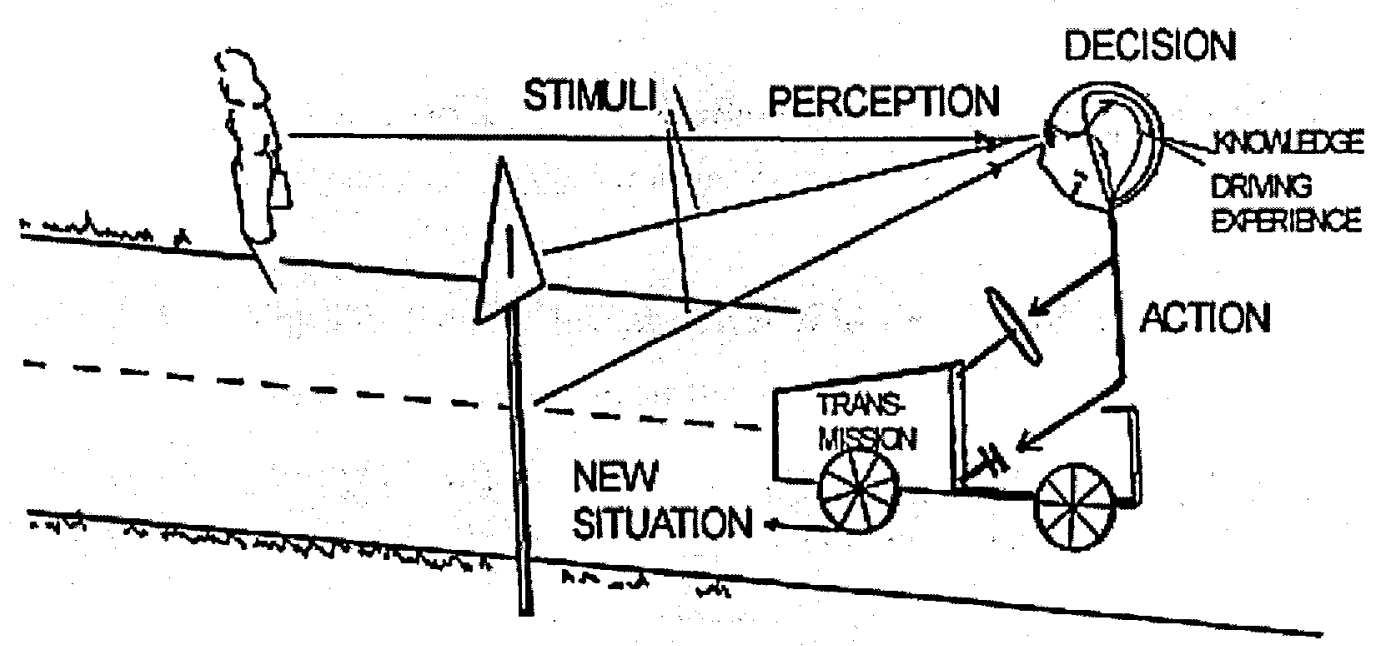

Figure 2.1 Basic components of driving (Cited from Pursula 1999) 


\subsection{Modelling Driver Behaviour}

In addition to describing the driver as a key part of the vehicular traffic in section 2.2.3, this section focuses on various elements of driver behaviour. Different types of driver behaviour models are critically summarised in (Michon 1985). This section also looks at cognitive, decision and mechanistic models as important aspects of driver behaviour. This section additionally explores models representing behaviour such as car following, lane changing, gap acceptance and overtaking within the general context of driving. Driver behaviour models draw from a diverse range of fields including, psychology, human factors research, behavioural sciences, statistics, control theories, amongst others.

\subsubsection{Elements of Driver Behaviour}

Michon noted in 1985 the extensive lack of research in driver behaviour modelling. $\mathrm{He}$ observes that such lack of research in this area can be attributed to a number of issues, including, failure to improve on the driver behaviour models of the sixties (e.g. car following) and a lack of interesting research ideas on driver behaviour. Concerning the future direction of driver behaviour modelling, he suggests that "we are heading for an intelligent, knowledge and rule based model of the driver that will be capable of dealing with a wide variety of realistic, complex situations..."

\subsubsection{Cognitive Models}

Michon is one of the proponents of using cognitive models in modelling driver behaviour. He has identified and described in detail, various types of driver behaviour models (Michon 1985). He proposes a hierarchical structure of driving behaviour divided into strategic (planning), tactical (manoeuvring) and operational (control). In this structure, planning and determination of goals is achieved at the strategic level, whilst selection of these goals is performed at the tactical level. At the operational level, actions are executed to achieve the planned goals and objectives. Tactical level decisions are critical in the support of operational goals execution. However, tactical level decisions are influenced by a number of factors including perception, driver behaviour, traffic situation, road geometry etc. To resolve this complexity in robotic 
navigation, separate modules supporting key decisions such as obstacle detection (Langer et al 1994), road following (Pomerleau 1993) are usually implemented. A major problem with this approach however is conflict resolution between modules. Since each independent module works on an individual task, different modules do not always have a common understanding of the overall situation. Despite this limitation, cognitive modelling using Michon's hierarchical structure is widely accepted to offer a sound basis for modelling driving tasks.

Drivers' cognitive processes such as reasoning can be represented using a production system framework (Michon 1985). This technique is particularly useful for modelling cognitive processes that can be expressed using three main components (1) A Rule Base (2) Working Memory (3) An Inference Engine or Interpreter. Within the context of AI and behavioural animation, (Funge 1999) has applied cognitive models to imbue characters/agents with knowledge and reasoning abilities that enable them to behave both autonomously and intelligently. In either driving simulation or computer animation, the development of cognitive models should involve modelling of perception, allowing individual driver agents to maintain a distinct representation of their environment.

Various other methods have been proposed in modelling driver behaviour. Some of these include rule-based systems, fuzzy logic, probabilistic models, hidden markov driver models and finite state machines. Rule-based systems provide a convenient and efficient way of representing human knowledge as part of the decision making process, in simple if-then cases. The cases require preconditions, which are often prescriptive, and these preconditions must provide an exact match in order for the rule to fire. This poses two problems in representing human driver knowledge into models. Firstly, a threshold relating to driver preferences such as preferred speed, preferred distance, etc. have to be defined. Often this definition is an ad hoc process in which the execution of the rules is greatly influenced by the unreliability in the thresholds. Secondly, rule-based systems suffer from inflexibility in adapting to global changes. Since rules are predefined locally, any global changes that were not considered in the predefinition, will require the formulation of new sets of rules to handle this new situation. The approach adopted in this thesis to remedy this problem involves 
allowing driver agents to become reactive/autonomous through perception and selfmodification of their local rules based on the perceived environmental situation.

The limitations of rule-based systems can also be improved by the incorporation of fuzzy logic systems (e.g. Seif El-Nasr and Skubic 1998). Here, imprecise information can be represented as linguistic variables allowing for an approximate match between the input and the rules. For example, an overtaking manoeuvre could emerge by combining rules with fuzzy information such as: if the current_forward_distance is less than the preferred_forward_distance and the target lane is free of vehicles, then execute the overtaking manoeuvre. In this example how do we define "less than" and "free of vehicles"? Less than is pre-condition defined in the rule system and free of vehicles is not very clear but we can define new semantics to handle this imprecision. Such representation within driver models is a very natural way of extracting decisionmaking heuristics from human experts. However, one of the main disadvantages of fuzzy systems is that they require hand tuning to choose suitable membership functions, leading to a time consuming and subjective process.

Probabilistic driver models aim to represent the inherent uncertainty involved in driving. This uncertainty derives mainly from lack of other drivers' intentions, lack of perception information and vehicle dynamics behaviour. Probability distribution approaches offer opportunities to model uncertainty in the perception and decisionmaking tasks. Such approaches have been successfully employed as part of driving simulation modules (e.g. Niehaus and Stengel 1994, Wright 2000a). The approach lends itself easily to quantifying traffic situations accurately, because of the availability of accident and traffic survey data. A probability sample of a given behaviour is obtained often from accident data and initialised for this behaviour. The probabilities are then fined tuned (using Bayesian technique) to calculate an output function based on an acceptable threshold. Probability approaches are often criticised for the mechanistic manner in which driver intentions are represented since the capturing of probability data is prone to calibration errors.

Hidden Markov Models (HMM) provide a better solution to modelling different driver states and attributes such as caution, aggression, experience etc. These attributes can then be used to measure driving intentions and the assessment of the 
associated risks. HMMs are easily transformed into supervised learning systems. Such systems provide the opportunity for autonomous behaviour, as individual drivers are capable of reacting and adapting to different situations. Hidden Markov Models increase the complexity in modelling driver behaviour since the behaviour of each driver in the simulation is modelled at micro-level. Driver behaviour models have also been developed using finite state automata (Booth et al 1993, Cremer and Kearney 1994, Cremer et al 1994). Problems associated with these models usually include difficulty in representing driving knowledge without resorting to its hard coding.

\subsubsection{Decision Making Models}

Driver behaviour models are influenced by decision-making and perception characteristics. Section 2.2.3 has already provided a brief discussion of the perceptual capabilities of the human drivers, with the intention of extending this discussion in chapter 3. Therefore this section will concentrate on decision-making. The representation of driver decision processes in traffic is challenging for a number of reasons. Firstly, decisions are influenced by the constantly changing traffic conditions, for example the behaviour of other drivers, vehicle performance. Secondly, driver decisions are affected by the driver's mood and inherent aggressiveness, e.g. tired drivers are likely to make poor or wrong decisions. Thirdly, the accuracy of decision-making is improved through accumulated experience, so more experienced drivers tend to make better judgements. Finally, it has been suggested that drivers' decisions are based on heuristics rather than optimised decision rules (Richard 1999).

The difficulty in modelling driver decisions has led to methods such as rule-based approaches (Moray 1986) to address driver risk taking behaviour; tactical driver behaviour (Sukthankaar 1997).

\subsubsection{Adaptive Control Models}

Adaptive control models describe driver behaviour in two contexts i.e. servo-control models and information flow control models. The use of servo-control models in describing driving is particularly useful in modelling the operational mechanisms of 
driver/vehicle interaction. The driver usually receives input signals of the vehicle and then steers the vehicle in response to these signals. An example of an enduring servocontrol model that has been extensively applied in driver steering behaviour is that of (McRuer et al 1977). However, these models have a distinct lack of integration between perception and vehicle control. In contrast, information flow based control attempts to model the driver by representing the various behavioural tasks as control structures or flow charts which are implemented as computer simulation (Kidd and Laughery 1964). Such models provide a framework to incorporate many types of behaviour, such as collision avoidance by continuously checking the visual information from the environment, reaction parameters, etc. as part of the driving task. Although growth in computer simulation has brought about increasing application of adaptive control models, such models still lack intelligence and learning capabilities because of their data driven approach.

\subsubsection{Car Following}

Vehicle following or what is commonly known as car following is one of the oldest and most fundamental driving tasks. Car following plays a very important role in understanding traffic flow (Rothery 1997). For example capacity estimates of single lane roadway are determined by the speed-spacing relationship of one vehicle following another. Such a relation assumes that the following and the followed vehicle are maintaining constant speed and constant spacing between them. This behaviour also considers many factors including perception, decision making, vehicle characteristics and control actions. Perception involves the processing of relevant visual information pertaining vehicle speeds, relative speeds, acceleration, looming optical flow, etc. On the other hand, decision making addresses interpretation of the information gathered in the perception stage using the driver's knowledge and driving experience. Perception and decision making lead to vehicle control with feedback from the environment guiding appropriate level of vehicle control. These processes are usually described in terms of perceptual-motor skills, with a particular focus on the understanding of information used by the driver in vehicle following. For a thorough review of this subject see (Brackstone and McDonald 2000). 
In the early stages of overtaking, unlike steady state vehicle following, the speeds of the following and lead vehicles are different. In this case the driver of the following vehicle infers from an increase in the visual angle of the lead vehicle, that the separation distance between the two vehicles is closing. In addition, the driver also uses motion perception (perception of relative velocities) as a function of time headway such that at short separation distance or short time headways, the driver becomes very sensitive to small changes in relative velocities. The driver will execute vehicle control using visual cues and speed control to maintain vehicle position within the roadway.

\subsubsection{Lane Changing}

The previous comments concerning overtaking are relatively simplistic because overtaking can be divided into a number of lower level tasks involving lane changing. Lane changing behaviour is a complex task compared to car following. This is because, in lane changing there are many considerations to be taken into account, for example, the behaviour (or motives of other drivers) in the lanes where the current driver is intending to move to must be carefully assessed. Also the desire to change lane is influenced by the driving conditions of the adjacent lane in which the current driver is intended to move into. Lane changing may have to be suspended if the traffic conditions do not permit it. It is therefore the case that lane changing behaviour can sometimes take longer from the intention to actual execution and completion of the task. An example of detailed lane changing behaviour is implemented in the PELOPS driver model (Ludmann et al 1997). The decision to change lane in this model is assigned a 'contentedness' factor, which considers the current vehicle's desire to change lane, along with a measure of its speed and the speeds of the other vehicles in the neighbouring lanes. In (Ahmed et al 1996) a lane changing model is developed as a sequence of three steps: 'decision to consider a lane change, choice of left or right lane and search for an acceptable gap to execute the decision'. These decisions are modelled within a discrete choice framework, but the mandatory lane changing model is not easily applicable in heavily congested traffic. Parameters for the discretionary lane changing model have also failed to be estimated. 
An inherent part of lane changing behaviour is gap acceptance. For example in lane changing a driver must assess the critical gap length of the target lane to which he intends to move to. Gap acceptance also occurs at intersections where the driver must decide when it is safe to enter the intersection. This decision is influenced by factors such as conflicting traffic movement in the neighbouring lanes as well as the priority and perceived behaviour of other drivers.

\subsubsection{Validation of Driver Behaviour Models}

The validation of driver behaviour models take many forms ranging from obtaining data in controlled environments such as a driving simulator (Groeger et al 1999), gathering field data with an instrumented vehicle (Brackstone et al 1999), video analysis of recorded traffic flow (Masroor et al 1997) and comparative studies based on empirical data extracted from related research literature (Wright 2000b). Particular driving tasks maybe validated by defining a similar task in a driving simulator, asking driver subjects to participate in the experiment and then comparing their performance with model prediction. For example in (Groeger et al 1999), the validation of speed and distance perception in the Leeds Driving Simulator was performed by recreating a recorded real-life scene of a roadway in the simulator and recruiting 100 driver subjects to estimate distances and speeds of target objects whilst in the simulator cabin. The studies showed a degree of underestimating and overestimating of both distance and speeds by the subjects under various experimental conditions. This approach suffers from the fact that simulated environments such as a driving simulator tend to encourage extreme behaviours since the subject knows there is no consequence associated with crashing. Hence these results are sometimes questionable.

In (Brackstone et al 1999) an instrumented vehicle equipped with forward pointing radar and driven on real roads by driver subjects was used to collect distance and speed data relating to the vehicle directly in front of the instrumented vehicle. At the same time each of the 7 subjects were asked questions relating to their perception of the distance and speed of a target. This data was used to validate the perception of closing and car following behaviour in a fuzzy logic based microsimulation framework. This approach is relatively more accurate than the simulated environment 
since the drivers are exposed to a real-life driving environment. However, like many real-life experiments it poses the risks of accidents (although the authors commented that every effort was taken to minimise task overload) and more importantly, it is difficult to make any rigorous statistical conclusion due to the small sample size.

Data extracted from video analysis provides a compromise between the previous two approaches to validating driver behaviour. In (Masroor et al 1997), traffic on a section of a roadway was recorded and then analysed with a traffic image processing tool (Maurer 1999) to extract speed distributions and vehicle trajectories. As the authors themselves acknowledged, the accuracy of the data is dependent on the calibration process of the tool and also the fact that the results cannot be applied generally to other road sections.

Sometimes it is unsafe or impractical to collect data using any of the previous techniques. Therefore, the review of relevant literature provides the opportunity to extract useful data to calibrate and compare results of driver behaviour models. This approach was adopted in (Wright 2000b) in which the author conducted an extensive study of the relevant literature to obtain empirical data for the configuration and evaluation of the different aspects of a driver behaviour model. It is important to bear in mind however that data obtained from comparative studies are influenced by issues such as lack of knowledge of the original setup, assumptions made, analysis techniques employed, etc.

\subsection{Traffic Generation for Driving Simulation}

Traffic generation for driving simulation is an important requirement, especially for fixed base or motion based driving simulators ${ }^{1}$. In general the complexity of the synthetic driving environment is dependent on the intended fidelity of the simulator or simulation. In driving simulators, realistic representation of the traffic environment and entities is essential to produce believable interactions with the human driver subject and thus improve driver training and experimental investigations relating to

${ }^{1}$ Traffic simulation models tend to refer to the software architecture of the driving simulator whilst driving simulators generally refer to the combination of a traffic generation module and fixed or motion base vehicle cabin. 
$\mathrm{road} /$ traffic safety. As a result, the development of traffic generation for driving simulators should consider the level of details, the database system that generates the synthetic or virtual environment and scenario modelling capabilities.

\subsubsection{Macroscopic Traffic Simulation}

A macroscopic traffic simulation model "describes the entities and their activities at a low level of detail" (Lieberman and Rathi 1997 p. 6) requiring less computational time. Also, macroscopic traffic simulation allows for higher level of abstraction and the ability to model large scale networks because of its aggregate description. However, macroscopic simulation models are low fidelity and often based on the deterministic relationships between the traffic entities. For example, network level traffic conditions, route choices, roadway environment, traffic flow etc. Typical examples of macroscopic traffic simulation models include NETFLO 2 and FREFLO (Lieberman and Rathi 1997).

\subsubsection{Mesoscopic Traffic Simulation}

Mesoscopic traffic simulation models are mixed fidelity types. These models describe some elements of the traffic system at a high level of detail but represent the interactions at a relatively lower level of detail than microscopic traffic simulation models, below. For example, road network and traffic dynamics are represented at an aggregate level, whilst individual vehicle movements and interactions are represented in greater detail (Ben-Akiva et al 1998). In this way, mesoscopic traffic simulation allows flexibility in the design of traffic systems because it supports trade-offs between the accuracy of the model and computational efficiency. Examples of these types of models include, NETFLO 1, DYNASMART, and TRANSIMS (Lieberman and Rathi 1997).

\subsubsection{Microscopic Traffic Simulation}

Microscopic simulation offers the most general method for realistically describing and analysing the nature of vehicular traffic. Microscopic simulations are high fidelity models capable of representing the individual characteristics of the traffic elements 
such as driver, vehicle and road network at a high level of detail. Representation of such characteristics within microscopic simulations produces realistic interactions, provided these characteristics have been accurately described. In (Druitt 1998), the author suggests that microsimulation representation of the apparent randomness of road traffic can be described using simple rules of car following, gap acceptance and vehicle kinematics. This approach allows for situations or events that are not explicitly modelled to occur as a consequence of the modelling process, for example, queuing at traffic signals or junctions, lane changing, shock waves, etc.

In addition, sub-microsimulation approaches based on methods such as Cellular Automata (CA), nanosimulation, fuzzy logic and prescribed rules are allowing better individual driver behavioural characteristics and complex interactions to be obtained. In Cellular automata (Brilon and Wu 1998, Barlovic et al 1999, Neubert et al 1999), the microscopic description of vehicular motion is achieved by updating a set of local rules within a spatial lattice or cell. This allows properties and behaviour of complex traffic such as flow-density relationships and emergent traffic jams to be reproduced. The use of fuzzy logic microscopic simulation of traffic offers the opportunity to introduce uncertainties in modelling behavioural processes (McDonald et al 1997, Wu et al 1998). The authors have demonstrated a fuzzy logic description of car following behaviour using real driving data obtained to validate distance, speed and acceleration perception within their microscopic simulation model. Finally, in rule-based microsimulation, driver behaviour is described by simple rules representing for example car following and lane changing behaviour (Lehmuskoski and Niittymäki 1999). These rules typically describe the decision processes of drivers in the form of if, then statements and ensure that rules only fire if their conditions evaluate to true.

Microscopic traffic simulation offers a number of benefits. As already discussed, it allows for detail of the system to be modelled, thereby producing interactions consistent with real life observations of traffic system. However, because of the need to represent traffic entities at this high level of detail, these models require a relatively large amount of computational time and memory. Coding such systems also poses significant challenges, as accurate models of network, driver and vehicle are required. Finally, the validation of the individual components of microscopic simulations can 
be difficult, since the validation process must rely on those points made in section 2.3.4.

There are various types of microscopic traffic simulation models or tools. The SMARTEST ${ }^{2}$ (Simulation Modelling Applied to Road Transport European Scheme Tests) project identified 58 tools available worldwide. Typical examples being PARAMICS, HUTSIM, MITSIM, INTEGRATION, SIDRA, NETSIM, FRESIM and CORSIM, TRAF-NETSIM etc.

\subsubsection{Agent-Oriented Traffic Simulation}

The definition of agent means different things to different people. In (Wooldridge and Jennings 1995), the notion of agent is distinguished in two ways - 'a weak notion of agency' describes a hardware or software-based computer system and includes properties such as autonomy, social ability, reactivity and pro-activeness. The second definition describes 'a stronger notion of agency' which is more AI oriented and usually represents a computer system that can exhibit human like properties such as knowledge, intentions, emotions as well as mobility. Such properties can prove useful in facilitating the development of adaptive intelligent agents where 'adaptive' is exemplified by human-like abilities (ALife), 'intelligent' denotes behaviour based on $\mathrm{AI}$ and 'agent', itself, indicating the software paradigm. Within such a context agents typically exhibit properties such as reactivity through perception of the environments; goal-orientedness identifying of complex tasks to achieve a goal plan; social ability achieved through interaction with other agents within their environment and autonomy demonstrated by agents operating independently in executing certain tasks. The behavioural rules that model these characteristics are defined for individual agents, allowing them to interact on an individual basis, with complex group behaviour emerging as a consequence.

In recent years, a growing number of agent-based paradigms have been applied to traffic simulation (Erol et al 1998, Fernandes and Oliverira 1999, Dia and Purchase 1999, Champion et al 1999). These approaches, like microscopic simulation, attempt

\footnotetext{
${ }^{2}$ SMARTEST Project Deliverable D3, August 1997, European Commission, $4^{\text {th }}$ Framework Programme, Transport RTD Programme, Contract No: RO-97-SC.1059
} 
to model detailed individual behaviour e.g. network, driver, vehicle and their interactions over a specific period of time but, unlike their microsimulation counterparts, agent-based simulation supports intelligent autonomous behaviour. Also, agent-based traffic simulation readily captures the concept of traffic flow as a complex emergent behaviour resulting from the local behaviour and interactions of individual traffic participants within a dynamic environment. Furthermore some agent-oriented traffic simulations are capable of representing detailed individual and group behaviour as part of traffic signalisation and control in distributed infrastructures (Manikonda et al 2000). By using agent technology, these applications offer several benefits including flexibility and intuitive software design and reuse, improvements in realism by describing properties of the system similar to real-life characteristics. For example, local interaction based on individual agent's perception, decision and action, leading to emergent global behaviour.

\subsubsection{Synthetic Driving Environments}

The synthetic driving environment (also commonly referred to virtual driving environment) usually consisted of a database responsible for the creation of road geometry and its associated logical connections. Such a database is in turn supported by various $3 \mathrm{D}$ visualisation graphics and terrain information which is required to drive the movement of the traffic entities in the environment. In (Papelis, and Bahauddin, 1995) the database for traffic simulation in the Iowa Driving Simulator is described. This provides the logical road network information of the virtual driving environment. In many cases the synthetic driving environment is generated by various means including commercial 3D modelling applications such as Multi-Gen, (as used in the Leeds Advanced Driving Simulator), Open-Flight, script-based definition (van Wolffelaar et al 1999) and graphical user interfaces - GUI (Bayarri et al 1996). Script definition and GUIs are discussed in the next section.

Although realistic representation of surrounding traffic in many driving simulations is also important, it is argued later in this thesis that a reduced level of detail in terms of simple animation can often be sufficient to achieve realism in visualisation. This provides beneficial tradeoffs between computational time, realism and efficiency in the visualisation of the synthetic environment. 


\subsubsection{Scenario Modelling}

Scenario modelling is a fundamental element in driving simulation. It involves definition of various features within the synthetic environment, including initialised parameters and possible interaction of the traffic entities. Papelis (1996) identifies three possible levels of scenario authoring that attempt to address some of these issues. Examples of these levels include hand-tuned scenario authoring where direct access and modification of the source code is required to create various scenarios; parameterized scenario authoring allows some flexibility by allowing the specification of initialised parameters such as preferred speed, preferred distance, preferred lane position etc. for the different scenarios, each defined at the start of the simulation. Finally, the authoring with dynamic coordinators level essentially assigns simple instructions to modules (BMO - Behaviour Modification Options) responsible for high level behaviour instead of these high level tasks being scripted by the scenario creator.

An example of hand-tuned scenario authoring is described in (van Wolffelaar et al 1999). Driving simulation scenarios, for example, are based on a Scenario Specification Language (SSL) which is used to define road network parameters and behaviour data of the traffic entity. The SSL defines the syntax and semantics of the scenario by allowing the user to specify and modify scenario scripts at the programming level. An example of parameterized scenario authoring using a range of interactive facilities to configure different scenarios is demonstrated in (Sukthankar 1996). Since the entities (referred to reasoning objects) in the simulation are capable of intelligent and autonomous behaviour, the scenario generation process allows for the customisation and evaluation of scenarios at various points of the simulation. A hybrid of the parameterized and $B M O$ scenario modelling is introduced as an attempt to provide the autonomous reactive behaviour associated with intelligent agent-based simulations. Cremer et al (1994) described a scenario control infrastructure employed in the Iowa Driving Simulator that realistically creates and controls the autonomous and complex reactive behaviour of objects in the simulation using state machines. These state machines direct on-line scenarios as they are executed, by switching the states of individual entities and allowing them to react naturally to events without user intervention. 


\subsection{Chapter Summary}

The review carried out in this chapter has identified a variety of concepts and approaches for vehicular traffic modelling. In this context, basic traffic flow theories have provided the author with better understanding of procedures of traffic flow, knowledge of developing accurate and realistic traffic models and an informed scepticism in interpreting computer model results. Also behind the apparent complexity of vehicular traffic modelling lies an ordered approach. This is succinctly put by Druitt in (Druitt 1998, p 482) as: “...behind the apparent randomness of road traffic, lies a complex order based on simple rules of car following, gap acceptance and vehicle kinematics. These can produce complex behaviour over a wide area when traffic densities are high. One reason for this complexity is that high density traffic is prone to chaotic processes which are sensitive to small disturbances.". This behaviour is better described by agent-based or microscopic simulation because these approaches offer opportunities to describe the detail of individual traffic components.

In traffic simulation, the review has demonstrated the effectiveness of simulation to a wide range of complex problems. In particular, traffic simulation offers a suitable computational framework to represent drivers, vehicles and road networks as well as capture their varied interactions. In addition, traffic simulation provides an opportunity to estimate quantitative data and allow the analysis of these data statistically. When such analysis is augmented with animation capabilities, it provides credible information to the decision maker in terms of understanding the behaviour of the system being modelled and implementation of any design improvements.

The discussion on driver behaviour modelling is essential for formulating an intelligent cognitive driver model. Such a model would have to draw from the various positive achievements of those reviewed in section 2.3. Also, as noted by Michon, driver behaviour modelling based on a rule-based system such as (Newell and Simon 1972) or production rules (Anderson 1996) seems to offer the most general representation of intelligent human activity pertaining to driving.

The validation of driver behaviour models within traffic or driving simulations is critical but at the same time challenging. It is critical in the sense that if virtual drivers 
are to exhibit realistic human behaviour then those characteristics that define human behaviour must be incorporated and proved to be believable. The challenge of this is reflected in the difficulty of designing experiments to obtain objective measurements of human driver behaviour. The four methods identified in section 2.3.4 have each got their advantages and disadvantages but they offer a framework in which to perform validation work.

What is not clear however, is the extent to which vision has played an important role in driver behaviour modelling. Although vision is recognised to contribute $90 \%$ to the driving task, it is typically only investigated in experimentation analyse and few attempts have been made to explicitly model the features of human vision for traffic/driving simulations. The next chapter devotes time in discussing the key features of visual perception modelling techniques, in particular identifying vision mediated behaviour in vehicular traffic. 


\section{CHAPTER 3}

\section{REVIEW OF VISION AND VISUAL PERCEPTION MODELLING}

\subsection{Introduction}

This chapter revisits various aspects of driver behaviour introduced in chapter 2 , but from a driver vision perspective. Firstly, the chapter discusses biological vision to establish a basis on which artificial or non-biological vision models can be developed. Secondly, the chapter presents an overview of existing vision models within software environments, additionally considering approaches, techniques and tools used to support the development of vision models.

\subsection{A Basis for Biological Vision}

A study in vision should start with a basic understanding of biological vision. This section is concerned with human vision. Human vision is a fundamental sense and dominates the other senses - smell, touch, taste and sound. However, biological vision and the way the brain manages visual information is immensely complex and as yet not fully understood. It is therefore not the intention in this section to discuss in detail the anatomical function of biological vision systems. Therefore, taking a simplistic view of the physiology of the human visual system, a cross section of the human eye is shown in figure 3.1. The eye plays a significant function in encoding initial visual information and is therefore described in the following sub-sections. The intention of the discussion in these sections is to establish a knowledge framework on which to develop a realistic vision model that utilises this knowledge.

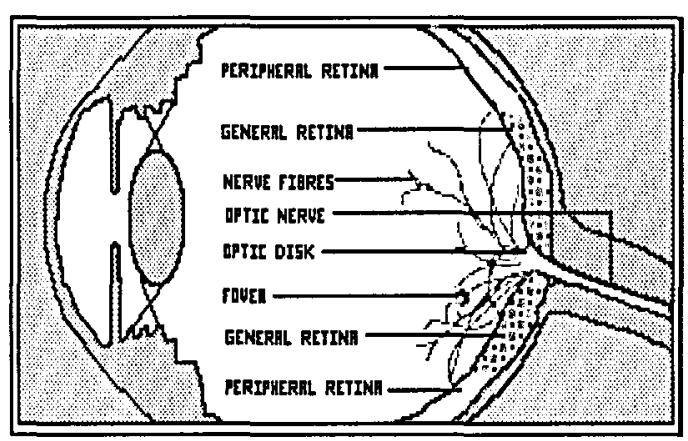

Figure 3.1: A cross sectional structure of the human eye and optics (reproduced with permission from Mather 2001) 


\section{CHAPTER 3}

\section{REVIEW OF VISION AND VISUAL PERCEPTION MODELLING}

\subsection{Introduction}

This chapter revisits various aspects of driver behaviour introduced in chapter 2 , but from a driver vision perspective. Firstly, the chapter discusses biological vision to establish a basis on which artificial or non-biological vision models can be developed. Secondly, the chapter presents an overview of existing vision models within software environments, additionally considering approaches, techniques and tools used to support the development of vision models.

\subsection{A Basis for Biological Vision}

A study in vision should start with a basic understanding of biological vision. This section is concerned with human vision. Human vision is a fundamental sense and dominates the other senses - smell, touch, taste and sound. However, biological vision and the way the brain manages visual information is immensely complex and as yet not fully understood. It is therefore not the intention in this section to discuss in detail the anatomical function of biological vision systems. Therefore, taking a simplistic view of the physiology of the human visual system, a cross section of the human eye is shown in figure 3.1. The eye plays a significant function in encoding initial visual information and is therefore described in the following sub-sections. The intention of the discussion in these sections is to establish a knowledge framework on which to develop a realistic vision model that utilises this knowledge.

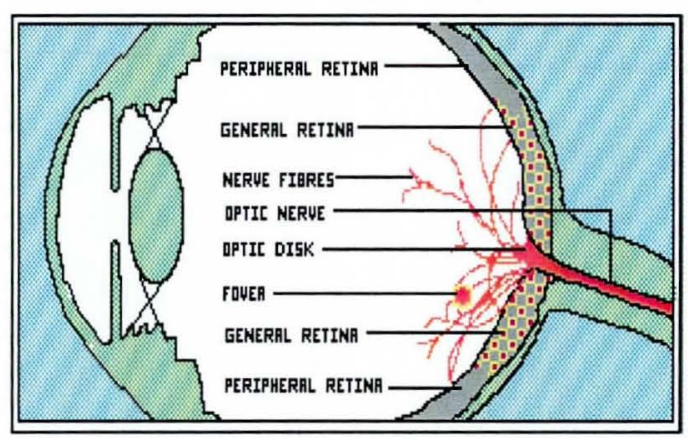

Figure 3.1: A cross sectional structure of the human eye and optics (reproduced with permission from Mather 2001) 
In order to understand the basic physiology and mechanisms of biological vision, Wandell (1995) has provided a framework in which to study vision by decomposing it into three key processes of image formation, image representation and image interpretation. This framework provides a structure for integrating elements and concepts of biological vision into non-biological vision models.

\section{Image Formation}

In image formation, light from a source is focussed at the retina by the cornea and lens to form an inverted retinal image. This is a linear process and is simplistically analogous to image formation in a photographic camera. Image formation is the first level of visual information acquisition which is performed wholly by the eye. The light sensitive elements of the eye transform the retinal image into neural signals which are transmitted through several visual pathways. These initial stages of image encoding influences the way other visual processes infer about objects. Therefore, understanding the process of image formation is critical in establishing the capabilities and essential limitations of human vision.

\section{Image Representation}

The second component of Wandell's framework is image representation. Image representation in human vision is performed between the retina and visual cortex and occurs within several visual pathways, with each pathway responsible for specific analyses of the retinal image. For example, tracking of object movement, image contrast, multi-resolution representations, image discrimination, all have specialised pathways. Within the retina itself there are five layers; photoreceptors, horizontal cells, bipolar cells, amacrine cells and ganglion cells. The photoreceptors layer in the human eye contains 100 million rods and 5 million cones in each eye (Wandell 1995). The rods and cones are spatially arranged in mosaic layout and this spacing is important to achieve visual acuity. For instance, widely spaced photoreceptors will lead to a significant variation in the retina image with reduced visual acuity. The rods and cones are mainly responsible for converting visual images into different intensity signals, transmitting copies of the signals to several other specialised neurons after the initial visual information has been encoded. The horizontal cells are connected to the rods and cones as well as with each other and are responsible for sending processes laterally between adjacent receptors. In order words, the horizontal cells along with 
the photoreceptors send signals to the bipolar cells. The bipolar cells then combine these signals which are then propagated through the amacrine cells and optic nerve, into the visual cortex.

Between the retina and visual cortex, the optic nerve fibres from each eye terminate at two separate parts of the brain in the thalamus called the Lateral Geniculate Nuclei (LGN). Figure 3.2 shows the projections to the LGN of the left and right visual fields. The optic nerve fibres from each eye's retina arrive at the LGN on the same side of the head but each projection of the visual field occurs on opposite sides of the LGN. This complex organisation allows the left LGN to receive visual information about the right visual field and the right LGN to receive visual information from the left visual field.

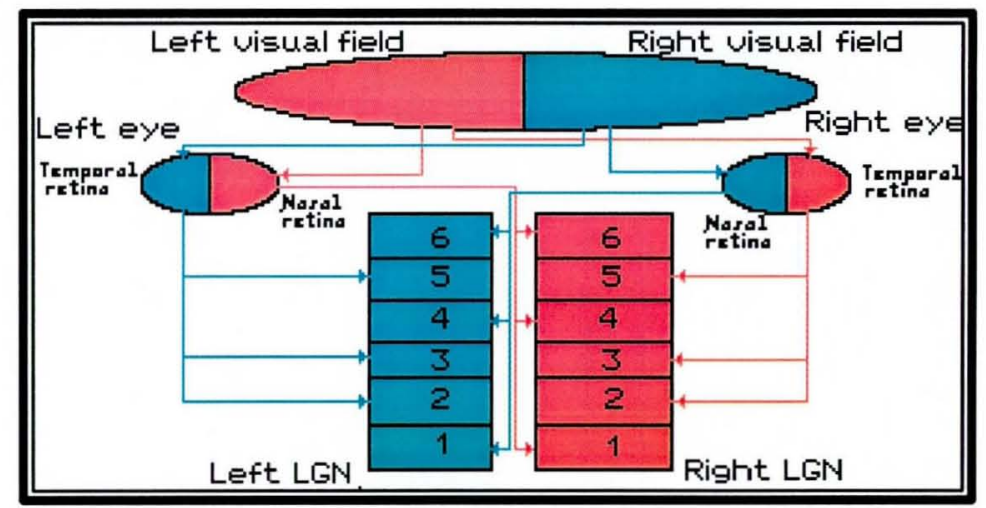

Figure 3.2: Projections of visual field in LGN (reproduced with permission from Mather 2001)

The visual signals from the retina and LGN arrives in the visual cortex which is made up of five areas labelled V1, V2, V3, V4 and MT (or V5) as shown in figure 3.3a. Of these five, V1 is the largest and the first region where all visual information enters. The V1 area consists of cells that are specialised in dealing with different orientations and directions of different stimuli. Figure 3.3b shows the architecture of the V1 cells. Noting the orderly arrangement of cells in the V1 region of a primate's visual cortex, Hubel and Wiesel (1962) observed 'orientation columns' in which individual cells' response is based on orientation turning or preference i.e. cells respond to stimuli of different orientation. In addition, they found 'ocular dominance' columns in which cells responded to dominant stimuli presented to either eye. That is, cells response in 
the left or right eye show no change for vertical movement but alternating dominance in horizontal movement. Although, the organisation of the cells and specialisation of the visual pathways provide an excellent framework for developing a computational model of visual perception, these complex pathways are still the source of much current experiment and speculation.

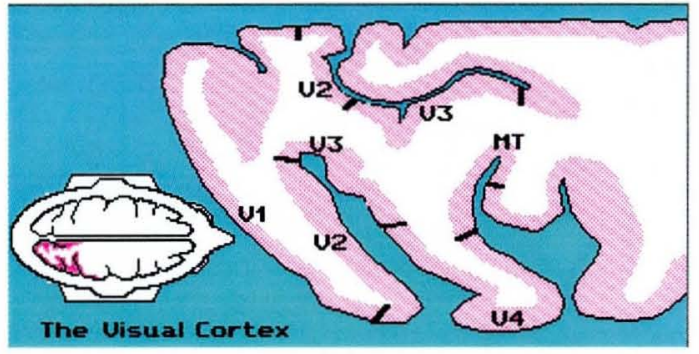

(a)

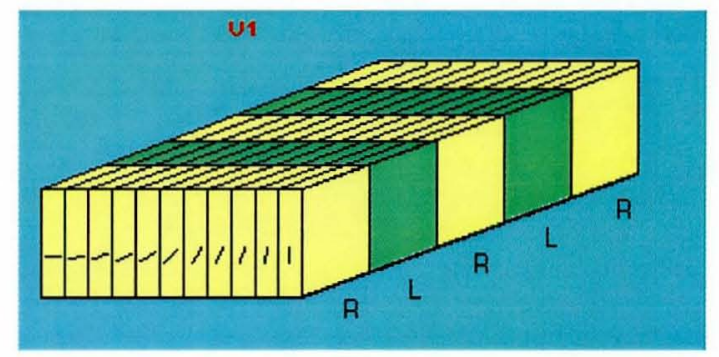

(b)

Figure 3.3: (a) The visual cortex and (b) V1 architecture of the visual cortex (reproduced with permission from Mather 2001)

\section{Image Interpretation}

The final component in the decomposition of biological vision is image interpretation. In terms of human vision image interpretation concerns visual inferences to describe object properties. This process occurs predominantly within the visual cortex and deals with visual perception such as colour, stereopsis, visual attention, motion analysis, depth perception, learning, knowledge and behaviour. Due to the immense complexity of the mechanism of the visual cortex in representing and interpreting images, the work reported in this thesis will be limited to the fundamental understanding of retinal image processing and the formulation of algorithms that capture some of the key features of visual perception.

\subsection{Computational Visual Information Processing}

By using a computational approach, the three processes of image formation, image representation and image interpretation can be combined to analyse and understand how visual information is processed for visual perception. Perceptual information flow has often been described using both bottom-up and top-down approaches. Although this classification is rather arbitrary in the sense that in real-life, there is crossover between the two, it nevertheless provides a framework in which information 
flow can be described qualitatively. For example, the image formation stage of perception can be formulated using a bottom up process by analysing how response properties of visual neurons and the connectivity between them are shaped by the visual inputs.

\subsubsection{Bottom-Up Perceptual Information Processing}

In the bottom-up approach, visual information processing is described as a hierarchy of representations. Providing a caricature description of the bottom-up or pure vision approach, Churchland et al (1994) lists the three core principles: (1) the visual world describes vision as a scene recovery process, the eye captures a complete image, this is processed in the retina and the information is later used to perform visual tasks. Therefore, what we see is a complete representation of the visual scene. (2) The Hierarchical processing principle says information processing occurs in distinct stages, from retinal image processing to the LGN, and finally to the higher cortical stage. Each of these stages has a specific function and is responsible for extracting specific features of the visual scene. (3) Dependency relations address the information flow in the visual process. Here, information flows from bottom to top, with higher level processing depending on lower ones but not necessary the other way round. This perceptual organisation of vision is often linked to Marr's computational theory of vision. The theory describes the complexity of visual information processing as computational theory, representation and algorithms (algorithmic level) and hardware implementation (Marr 1982). Computational theory addresses the underlying problems of information processing which requires a precise definition of the goals (what is present in the scene, and why it is appropriate, the processes for extracting the information) and the constraints associated with these goals. Representation and algorithms concern the exact choice of computation in terms of information representations and algorithms that control them. Finally, implementation addresses the physical realisation of the algorithms either in hardware or software.

The main criticism of bottom up approaches is usually directed at these processes being data driven with intractable problems of processing the immensely complex human vision system. As such they require a myriad of special programs and huge computing power. However, the bottom-up view provides a logical and computational 
framework which can be used to develop approximate synthetic vision models. Also, the guiding principles of the bottom up approach enables processes to emerge as a result of modelling capabilities of individual elements within the vision system. Furthermore, current advances in computing technologies e.g. parallel processing are making it easier to manage computational complexity using the bottom up approach

\subsubsection{Top-Down Perceptual Information Processing}

The premise of top-down vision is that what we see is only a partial representation of the whole scene. More formally, the scanpath theory (Stark et al 2001) suggests that a top-down internal cognitive model of what we see controls not only our vision, but also drives the sequences of rapid eye movements and fixations, or glances, that so efficiently travel over a scene or picture of interest. This theory is supported by the Gibsonian view of visual perception (Stark et al 2001). Gibson's theory studied ecological optics aiming to determine how the brain processes information about the environment. He suggests that the function of our perception process is to extract from the external world those properties that are required to perform a particular task (Gibson 1950, 1966, 1979). His computational paradigm considers higher order variables such as ambient energy, proportions, ratios, etc. In addition, Gibson's theory reduces computational costs because only necessary properties of the world are extracted. This differs from Marr's proposal in which reconstruction of the 3-D world from 2-D images has proved nontrivial and computationally infeasible.

Although a top down approach of visual information processing reduces computational costs through the application of internal cognitive maps or the use of precisely defined rules, this has a number of drawbacks. Firstly, the behaviour of humans or robots using a top down approach is limited to the information contained in their cognitive map. Encountering situations outside their parameters leads to unpredictable behaviour. Secondly, the complexity in information processing requires a large number of rules or detailed representation of the cognitive map to be built. This will often lead to conflicting operation of the parameters within the rules. In addition development of a cognitive map can be time consuming. 
The mechanisms of biological vision have several implications on the formulation of artificial or non biological vision systems. Firstly, vision is a diverse and multidisciplinary field using ideas from biology, psychology, mathematics, physics and engineering. Bringing these fields together require computational and experimental methods to analyse the various visual processes. For example, computational methods provide a means of characterising input and output relationships by calculating the transformation of any input image into the retina. Secondly, image representation draws from behavioural studies involving experimental methods to understand processes such as object detection, simple recognition and motion perception. Therefore, applying the knowledge gained from the study of biological vision offers the possibility of building realistic computer vision algorithms. Sections 3.4 and 3.5 discuss the implementation of non biological vision systems that benefit from computational and behavioural methods.

\subsection{Synthetic Vision for Autonomous Agents}

A working definition of synthetic vision adopted in this section refers to non biological vision developed to support autonomy of agents. 'Autonomous agent' is used here to refer to an entity capable of operating in its world without direct external intervention. Examples of autonomous agents include robots, unmanned ground vehicles and virtual drivers. A further distinction is worth noting between synthetic vision for the real world (real/active vision) and synthetic vision for artificial life applications (perception in virtual worlds). The application of synthetic vision to the real world is concerned mainly with building computational models of visual functionality in humans as well as machines that emulate them (Cantoni et al 1997). Research work in this area falls under the broad category of machine vision or computer vision, drawing from diverse contributions from robotics, image processing, artificial intelligence, pattern recognition, visual knowledge representation and cognitive processes such as reasoning and learning. The general problem with real world applications of synthetic vision, e.g. robotic vision is that its allied techniques and mechanisms are often complex, computationally intensive and not easily transferred to modelling vision within synthetic environments. 
Synthetic vision for virtual environments was first introduced in (Renault et al 1990) offering the opportunity to enhance the realism of simulated behaviour by modelling simple and limited perceptual capabilities such as agents' field of view, object detection and obstacle avoidance. The essential principle of synthetic vision is to allow the agent to render the scene from its viewpoint and use processed retinal images to guide local navigation. This principle is inspired from biological vision systems in which the vision system acts as a filter to limit perceptual processing by eliminating unnecessary information. For example, visual input information is captured by the eye in a narrow depth of field and eye saccades ensure specific visual objects or regions of the environment are sampled sequentially. Both the capabilities and limitations of the human vision system should be taken into account when developing vision models to enhance the behavioural realism of the agents.

\subsection{Visual Perception in Virtual Environments}

Before discussing the applications of synthetic vision in virtual environments, it is worth mentioning the importance of Artificial Life (ALife). In general, 'virtual environment' is defined as a three dimensional computer generated world in which both static and non-static objects exist, for example, buildings, sky, humans, vehicles, etc. The creation of Artificial Life in virtual worlds enhances the visual fidelity of the environment by invoking feeling of realism and intrigue, since agents can exhibit behaviour characteristic of natural living systems (Langton 1989) such as autonomy, adaptivity, etc. Thalmann-Magnenat and Thalmann (1994) provide a comprehensive list of virtual human characteristics in virtual environments. Furthermore, ALife in virtual environments also contributes immensely in the fields of entertainment, animation, multimedia, human factors analysis, virtual prototyping and education.

Various models of visual perception have been developed and applied in the area of ALife to help facilitate complex autonomous behaviour within virtual environment. In these systems, vision is an important part of the interaction between agents, for example, Boids (Reynolds 1987), Polyworld (Yaeger 1994), Virtual Humans (Thalmann 1995), Artificial Fish (Tu and Terzopoulos 1994, Terzopoulos et al 1996), Silas T. Dog (Blumberg 1997) and Creatures (Grand et al 1997). Many of these virtual environments have been developed to explore biology and evolution type 
behaviour, typically using simple sets of logical rules or other computational techniques to generate wide ranging emergent phenomena, e.g. self-creating, selfevolving, self-controlling that are useful in behavioural animation and virtual reality. The following sections provide a summary of some of the relevant synthetic vision models that support autonomous navigation within virtual environments.

\subsubsection{Boids}

In the earlier and groundbreaking work of Reynolds' "Boids" (Reynolds 1987), he demonstrated the flocking of birds or 'boids' by allowing individual simulated boids to sense their local environment and avoid collision with "nearby flockmates". Within their environment, boids have direct access to the geometric database that describes the exact position, orientation, and velocity of all the objects in the environment. Although the direct access or direct sensing method provides a fast way of extracting information from the environment its main criticism is that it essentially amounts to 'perfect global vision'. Obstacle avoidance behaviour in boids is based on a 'steer-toavoid' mechanism. This engages a behavioural controller to compute the closest obstacles directly in front of the boid that pose a collision threat. This enables the boid to re-orient itself to avoid crowding local flockmates, whilst steering towards their average heading.

\subsubsection{Polyworld}

The organisms in PolyWorld (Yaeger 1994), use a horizontal vector of pixels to generate a rendered view of their three-dimensional environment. Pixel outputs are connected to a neural network, with neurons specialised in vision of different colours and several vision related navigation routines such as, forward motion, turning and focusing. The idea of using neural networks i.e. an interconnected assembly of processing elements, units or nodes, whose functionality is loosely based on the mammalian neuron is a very powerful one. Forward motion and turning are achieved by activation of the organisms moving and turning neurons respectively. Focussing allows the organism to control its horizontal forward field of view to look at the world in front of it, whilst navigating. The focusing mechanism is implemented by mapping the focussing neuron to a number of possible angles. Although, this approach 
demonstrates an advanced combination of synthetic vision and internal representation (map) of the environment to plan navigational paths, it is limited by the fact that the organisms have only a one-dimensional retina and therefore much of the threedimensional information from the environment is lost during vision.

\subsubsection{Creatures}

In the commercially successful Creatures game (Grand et al 1997), vision is given to the creatures by activating a neuron representing particular types of objects present in the creatures' field of view. This approach borrows heavily from "semi-symbolic approximation techniques" used in neural network processing (Pierzchala and Rodanski 1993) e.g. vision neurons are activated when an object is present within the creature's line of sight. The technique offers Creatures the ability to learn from their environment and adapt to different situations. Learning in vision based neural networks is achieved through training, which could be either supervised or unsupervised. The training process is iterative and involves comparing the input signals with desired output to determine weights representing various connections of the network. The error between input and desired output is fedback until the network matches the ideal performance of the Creatures' learning system.

However, vision based neural networks have a number of problems. Salomon (1996) provides a good discussion on the general limitations and requirements in using neural networks techniques for autonomous agent behaviour. For example, training an agent with pre-selected data will lead to errors when the agent encounters unfamiliar data or in some cases will require further retraining. In addition, since neural network based vision models are implemented as layers (input layer, hidden layer and output layer), they are limited by communications problems between layers as well as increasing complexity with increasing numbers of layers. Furthermore, learning in neural network based vision models needs to be incremental, as the agent interacts within the environment. 


\subsubsection{Silas T. Dog}

Blumberg also demonstrated the application of synthetic vision approach in Silas T. Dog, an autonomous animated dog situated in the ALIVE system - Artificial Life Interactive Video Environment (Blumberg 1997). A "perspective camera" renders a scene and the acquired image is used within a "motion energy" strategy to aid both obstacle avoidance and low-level navigation within the environment. There are several drawbacks with this approach highlighted by the author, including assumptions that exclude complexity of the virtual environment and optimisation of the navigation behaviour.

\subsubsection{Artificial Fish}

The perceptual capabilities of the Artificial Fish in (Tu and Terzopoulos 1994) consist of 'cyclopean vision sensor' and temperature sensor. The cyclopean vision sensor extracts visual information from the point of view of the individual fish using ray tracing. The field of view of the fish is limited to a spherical angle of 300 degrees with a sensitivity region defined around the fish by a bounding box. The temperature sensor provides measure of the water temperature. Both the vision and temperature sensors have direct access to the geometric database (e.g. geometry, material property) and details of the physical simulation (e.g. position and velocities of nearby objects). The fishes are able to avoid collision by testing for threat of collision with other obstacles within their sensitivity region. This region defines the range of safety and therefore violation of this by another fish or the current fish approaching a static obstacle will trigger certain navigation behaviours such as turn left/right, move up/down and pursue moving targets.

To resolve some of the problems associated with direct access to the database and physical simulation data used in Fish, the authors introduced animat vision techniques in (Terzopoulos and Rabie 1997). The principle of animat vision in their work is to model the visual capabilities of an artificial fish using photo realistic computer rendering techniques. For example, the latest version of their vision model allows the artificial fish to have two eyes where each is implemented as a coaxial virtual camera with projective geometry techniques providing the facility to transform $3 \mathrm{D}$ world information into a $2 \mathrm{D}$ retina image. Several active computer vision algorithms (e.g. 
colour indexing, image stabilisation using optical flow, etc.) are employed to support retinal image processing including realistic saccades, foveation, colour image processing, object tracking and binocular vision. This is a marked improvement to the direct sensing approach.

\subsubsection{Virtual Humans}

The virtual humans in (Musse and Thalmann 1997) avoid collision using two vision strategies which are based on line intersections (or ray tracing). Collision in the first strategy is anticipated by calculating the future positions of the virtual humans from their current positions. Impending collisions are prevented by priority rule assignment. The second strategy employs the same line intersections but the virtual human now avoids collision using direction changes. In (Thalmann 1995) virtual humans can move around their virtual environment by walking, jumping or climbing and can even recognise and track a tennis ball. Both locomotion and tennis playing behaviour are based on visual perception capabilities, figure 3.4. In the same way, navigation in (Noser et al 1995) occurs in two levels. The first level involves agents or actors directly employing synthetic vision based on ray tracing techniques to move locally. The second level utilises the concept of visual memory implemented as a '3D occupancy octree grid' to represent the structure of the environment. In this method, rendered pixels from the agents' point of view are encoded to occupy a voxel and these voxels are then compared at each simulation update to determine perceived changes within the environment. Therefore the actors rely on internal representation of the $3 \mathrm{D}$ environment to memorise changes in the environment rather than obtaining precise information.
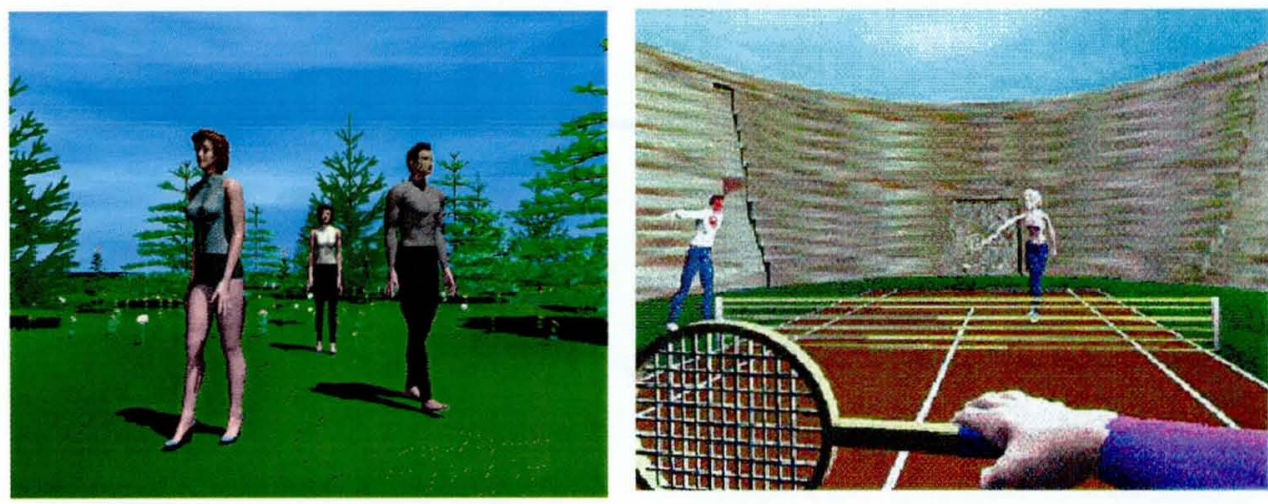

Figure 3.4 Autonomous virtual humans (reproduce with permission from VRlab, EPFL, Lausanne) 
However, although the use of octree structures in visual memory representation allows for efficient data reduction, one drawback with this method is that, it can prove time consuming in building the structure. In addition, when objects in the environment are complex it becomes difficult to achieve their detailed representation, leading to a rapid increase in required storage space.

Another realistic representation of vision capabilities in virtual humans is demonstrated in (Rabie and Terzopoulos 2000). Virtual human soldiers navigate within their environment through a number of control mechanisms which are based on animat vision. The virtual soldiers can perform head gazes, saccade, fixate on objects and track targets. Although the vision algorithms in the virtual human soldiers are based on robust image processing however, since the soldiers exist in rather simple environment, it is not clear how they can cope with for example, estimating speed or varying distance to target objects.

The vision approaches used in virtual humans markedly enhances the realism of their visual perception capability, since agents no longer have direct access to the virtual environment database. Furthermore, combining ray tracing and internal representation of the environment using visual memory is very appealing since it approximates real life image formation/registration and representation of visual information. It is therefore possible to consider adoption of some of the vision paradigms reported in these virtual environment to enhance autonomous virtual driver agents behaviour within traffic/driving simulations.

\subsection{Visual Perception Factors in Driving Simulations}

The characteristics of driver behaviour models were introduced in chapter 2, section 2.3. Since the behaviour of drivers is strongly influenced by their vision capability, significant new understanding may be obtained by incorporating vision models in driving simulations. Also in the following subsections, experimental studies of human driver perception of distance, depth, speed, velocity and motion are also examined. These are key concepts of virtual driver agents' perception capabilities because they introduce more realism and believability in their behaviour. 


\subsubsection{Driver Vision Models}

Section 3.5 reviewed the applications of various synthetic vision models for autonomous agents in virtual environments. These models, as already noted, enhance the behavioural realism of agents within these environments. However, within the area of driving simulation, visual perception is either usually ignored or addressed at an abstract level such that each driver agent has perfect knowledge' concerning the positions and velocities of other vehicles in the simulation. Although in (Wright 1998) the need for visual perception is recognised as a key component of the driver behaviour model, the lack of perception is nevertheless justified by the use of a probability distribution to effect overall behavioural uncertainty. Van Wolffelaar and van Winsum adopted a different approach in (van Wolffelaar and van Winsum 1995) by generating virtual traffic as part of a fixed-base driving simulator that allows users to interact with virtual driver agents in a synthetic environment. However, visual capabilities for the driver agents are assigned to the vehicles instead of the driver such that each vehicle is equipped with "sensors" that enable it to have perfect knowledge of distance, velocity etc. of all other vehicles.

The focus in (Cameron et al 1994) is on the implementation of robust traffic networks and traffic flow based on queuing theories. Vehicles move along the network by mapping themselves to slots on a queue. Each vehicle in the slot has direct access to other vehicles positions and speeds in that queue. Cremer et al (1996) introduced a database approach whereby vehicles can interrogate the database for traffic information such as road paths, traffic conditions etc. Although this method is simple and fast, vehicles still have direct access to global information, which is unrealistic. Another attempt to endow virtual agents with visual perception capabilities is given by (Tran 1995). This approach gives virtual driver agents the ability to avoid collision through a combination of trajectory interpolation and quadtree based calculation of "intervisibility".

The development and testing of visual routines in a driving simulator is described in (Salgian and Ballard 1998a, Salgian and Ballard 1998b). The routines are based on active image processing techniques e.g. image retrieval using shape and colour properties. These routines are embodied in a conceptual 'robot driver' or a scheduler 
which activates task specific visual behaviour such as detecting and stopping at traffic lights, detecting and stopping at signs, looming detection and car following. Since the intention of testing these routines in the simulator is to verify their performance to control an intelligent autonomous vehicle in the real world, the approach required far more powerful hardware resources and processing, compared to simple synthetic vision routines.

Whilst all of the above provide useful functionality within their own applications, none provide a well-structured framework for modelling vision mediated decisionmaking and other psychological issues within traffic simulation. Such a framework is explained in the following sections.

\subsubsection{Distance and Depth Perception}

The distinction between distance and depth according to Marr is that, distance is the "objective physical distance from the viewer to the object, usually measured from one of two eyes... and depth...the subjective distance to the object as perceived by the viewer" (Marr 1982, p.113). Furthermore, depth information, such as stereopsis and parallax, are useful cues to estimate relative distance to a target object. These factors are important in designing a vision system that requires binocular vision.

Various experiments have been conducted to study driver perception of distance (Groeger et al 1999, Recarte and Nunes 1998). For example, Groeger et al (1999) assessed driver perception of speed and distance in the Leeds fixed based driving simulator. In the distance study, subjects had to estimate the position of target objects that were presented to them on the simulator screen. The authors found that the accuracy of distance estimation improved when the subject is familiar with the objects and when they were nearer. The influence of speed and distance on estimated time to arrival is described in (Recarte and Nunes 1998). In conducting both field and laboratory experiments using human subjects, to analyse estimated arrival time as a function of speed and distance, one of the authors conclusions was that drivers' tend to underestimate distance, which in turn has influences on time to collision estimations. It was also recognised that drivers' performance was better in the laboratory experiment than in the field experiment. 


\subsubsection{Speed and Velocity Perception}

The common distinction of speed and velocity is that speed is a scalar quantity, whilst velocity is a vector. In (Brown 1955), the author argues that the perception of speed depends on the observer's travelling velocity. Therefore, faster speeds are perceived from environmental information whilst slower speeds are based on perception of consecutive sampling of changes in position.

The estimation of speed is important in many traffic situations. For example the perception of speed, especially that of other drivers usually influences the choice of travelling speed (Haglund and Åberg 2000). In other words, drivers tend to drive faster if others are perceived to be driving just as fast. In addition, perception of travelling speed is often a subjective task rather than a quantitative measure. In the same experiment carried out for the distance perception, Groeger et al (1999) observed that there were differences in the estimation of speed with sound and vision information. When only sound information was available, there was a significant overestimation of speed. They also noticed that when both sound and vision information are provided, vision information accounted for most of the higher speeds estimation and sound accounted for lower estimations. These observations have significant implication on driver behaviour and safety. For instance, many road accidents have been attributed to driver speeding and drivers' inability to accurately estimate travelling speed has also accounted for many rear end collisions.

\subsubsection{Motion Perception}

Motion perception is important in many aspects of vision, especially functions involving navigation, object tracking, control of orientation and movement. Referring to section 3.2, perception of motion is performed by motion detecting neurons, where specialised cells are responsible for detecting different types of motion. The significance of this is that a structure can be formulated to enable specialised (simulated) cells to analysis motion information.

Land (2000) identified two common cues required to guide vehicular navigation i.e. features displacement and optical flow patterns. Optical flow describes in general, the apparent flow (velocity field) of intensity in a sequence of images. It can be used to 
extract useful information from the environment including depth perception, direction of motion and also to perform motion detection, focus of expansion, object segmentation and time to collision. Features refer to points on the road, which induce direction of gaze. Land suggest that in many instances drivers tend to use tangent points (features) on the road to steer a vehicle especially on roads with bends but also noted that optical flow processing especially for distant regions plays an important role in directing gaze and hence vehicular control.

The effect of self-motion on the detection of object motion is studied in (Santos 1998) using an empirical approach to motion analysis. The experiments involved subjects detecting a looming and receding simulated target vehicle presented on a computer monitor. The subjects sat in a darkened room in front of a monitor and were shown real time simulation of the target vehicle. Since real-time changes in the environment created an immersion experience, perception of self-motion and object motion detection can be evaluated. The subjects had to respond to changes in their visual field by pressing the left and right mouse button at the point they perceived looming and receding of the target vehicle. The author found that visual motion (optical flow) played a significant role in the detection of the object motion. In other words the detection of the vehicle motion is higher with an increased in self-motion in the looming case. In addition, Boer (1999) described a statistical model based on looming information which also incorporates perceptual noise and 'forgetting' capabilities to predict object motion detection. The analysis of motion in Santos and Boer shows that describing direction of motion using looming and receding information is important for controlling vehicle headway and assessing time to collision judgements.

To complete this chapter, section 3.7 examines key vision techniques that are relevant to the development of the vision model in this thesis. The section combines various vision techniques for virtual driver agents situated in a synthetic driving simulation. As such only relevant computational vision techniques for both Computer Graphics and Machine Vision are reviewed in section 3.7. 


\subsection{Relevant Computational Vision Techniques}

This section examines a range of vision techniques employed in Computer graphics and Machine vision. In Computer graphics, ray tracing is a common approach used to render the scene from the agent's view point (e.g. Renault et al 1990, Thalmann 1995, $\mathrm{Tu}$ 1999). Machine vision techniques such as image processing provide robust algorithms for processing visual information (Jain et al 1995). Computer graphics and Machine vision cover very broad subjects and consequently only an overview of the relevant techniques to this project is provided.

\subsubsection{Computer Graphics}

Computer graphics provides the ability to build synthetic vision models to acquire information from the virtual environment by generating images from geometric primitives e.g. surfaces, cylinders, rectangles, and lines. The first stage of acquiring information from the environment can be achieved by using ray tracing techniques.

\section{Ray Tracing}

Ray tracing is a process by which an object or scene in 3D space can be reconstructed in the computer. This mechanism has several advantages over other methods like zbuffered rendering or scanline algorithm. Firstly, ray tracing performs well in generating computed images from three-dimensional perspective than $z$-buffered rendering techniques. Secondly, it can create realistic images of the scene through the ability to easily create reflections, refraction and shadows. However, incorporating this functionality can be practically complex and computationally expensive so the choice of the ray tracing method is crucial.

The underlying concept of ray tracing is to mimic the optics and object detection in the human vision system. This is illustrated in figure 3.5 with the view plane consisting of a grid of pixels. The imaginary light rays, $\mathbf{r}_{\mathbf{i}}$ will travel to infinity unless they hit an object. When this happens the light can be absorbed, reflected, refracted and/or transmitted. However, instead of light rays being reflected from the objects onto the eyes, a simplifying assumption in ray tracing is that, the light rays are traced from the view plane to the objects or into the scene (reverse ray tracing). This enables 
only those rays that contributed to 'seeing' or the formation of the image to be considered. Images are captured on the camera plane by ray-object intersection. The ray tracing process produces images that can be registered as either shaded colour (grey level or intensities) or binary pixel values.

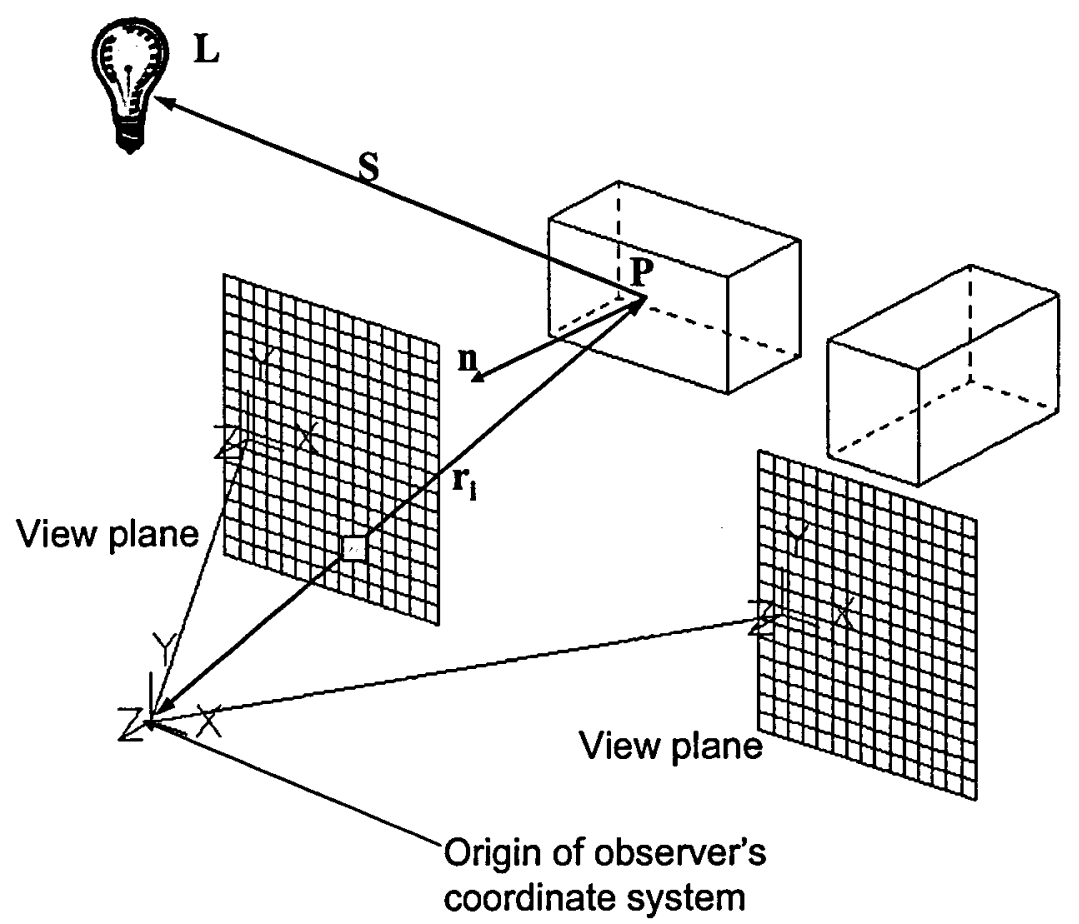

Figure 3.5: Vector notation. $S$ is directly pointing to the light source and hence has equation $S=L-P$ where $L$ is the light source, $P$ is the point of intersection and $n$ is the normal vector at this point. $r_{i}$ is the opposite direction vector of the ray.

\section{Colour Rendering}

The registered image corresponding to the $3 \mathrm{D}$ object in space contains a number of features that can be analysed. In the shading colour process, the image pixel corresponding to the hit point is set an image intensity or unique colour. In producing images of illuminated objects, the equation representing light intensity is given according to (Hill 1990) as:

$$
I=I_{a m b}+I_{d i f f}+I_{s p e c}
$$


Where: $I=$ is the light intensity as registered on the view plane and seen by the observer and $I_{a m b}, I_{d i f f}, I_{\text {spec }}$ are the ambient, diffuse and specular light reflections, respectively.

The ambient component is produced as a result of multiple reflections of light from more than one source and object. Diffuse reflection refers to some light being absorbed by the surface and some re-emitted uniformly in different directions. Specular reflection concerns the properties of surface e.g. shiny surfaces reflecting the entire incident light. Colour shading of the formed image is achieved by adding to each term in the equation rendering RGB values.

\section{Visibility Computation}

One of the problems in processing ray traced images is concerned with occlusion. When rays hit an object, all ray-object intersections will have to be tested to determine which object is visible. If several objects exist in the scene, some might occlude others and therefore, to compute visibility, occlusion will have to be removed. One common approach to solving this problem is to have access to the objects list stored in the geometry database and then compare the ray information with the stored information, to determine which object is visible. This is often referred to as direct sensing or direct access and is a fast and efficient way of improving computational time (Blumberg 1997). At the same time, direct sensing techniques suffer from the fact that as the complexity of the environment in terms of number of objects and geometrical properties increases, it becomes difficult and computationally challenging to build a realistic pre-stored data set of the scene.

An alternative to direct accessing is to apply mathematical morphology to remove occlusion. The operators in this area include erosion and dilation, and are typically applied to binary image analysis. A suitable erosion operator is the opening technique, which essentially involves taking (eroding) away the edges of images to split up connected images. Van Droogenbroeck (2002) provides two efficient opening algorithms, but only the first one is described here. This involves creating two matrices from scanning the input image. The first matrix denoted as $H O R[i, j]$ is scanned horizontally and vertically, whilst filling it with the distance values of the 
pixels corresponding to the right border of the object. The second matrix $V E R[i, j]$ is then formed by comparing the column values of the first matrix and the result is used to compute an opening in the form of rectangles.

\subsubsection{Machine Vision}

The underlying concept of Machine vision is to recover useful information about the scene based on its two dimensional projections. Scene or image information is usually acquired by a real camera. Machine vision techniques have a strong similarity to Computer graphics algorithms. For example, many surface and curve representation techniques are borrowed from Computer graphics. However, the major benefit of using machine vision algorithms is that they provide robust image processing, e.g. extracting and estimating features in images.

\section{Binary Image Processing}

Producing images that can be registered as shaded colour pose huge computational overheads since different colour intensities would have to be processed. An alternative approach is to record only the binary value of the pixels, thereby forming a binary image pattern. This approach is very appealing because it is relatively simple, its mechanisms are well established, it is faster than colour processing and it can handle logical operations better than intensity image processing can. The binary image pattern can then be processed using a number of techniques to determine features such as image size, position, orientation and projections (equations that follow are taken from Jain et al 1995). For example, the size of a binary image can be derived from its area using the zeroth-order moment, given by:

$$
A=\sum_{i=1}^{n} \sum_{j=1}^{m} B[i, j]
$$

Where, $B$ is a point on the camera plane and $n$ and $m$ refers to number of rows and columns respectively. $i, j$ represent the row and column indices, with $i$ counting downwards and $j$ left to right. Using the binary image of an object it is possible to determine its position (Jain et al 1995): 


$$
\bar{x}=\frac{\sum_{i=1}^{n} \sum_{j=1}^{m} j B[i, j]}{A}, \bar{y}=\frac{\sum_{i=1}^{n} \sum_{j=1}^{m} i B[i, j]}{A}
$$

This representation assumes a world coordinate system where the axes of the viewing plane are aligned with the coordinate system. The image is captured in the view plane (see figure 3.5 and figure 4.7). The coordinates of the centre of the image region is given by $\bar{x}, \bar{y}$.

\section{Segmentation}

Segmentation performs the extraction of important regions from images. The main techniques include region-labelling algorithm, statistical classification and boundarybased segmentation. Only the region labelling by connectivity method is described here. Region labelling provides a useful technique of extracting information from connected regions in images. The basic assumption of pixel connectivity in an image is that a pixel is spatially adjacent to other pixels and may share a common boundary. This spatial adjacency defines two types of neighbourhood notations, the 4neighbours and 8-neighbours. According to (Jain et al 1995), a pixel, $p$ with coordinates $[i, j]$ has neighbours as follows (modified from Jain et al 1995):

$$
\begin{aligned}
& N_{4}(p)=\{[i+1, j][i-1, j][i, j+1][i, j-1]\} \\
& \left.N_{8}(p)=N_{4} \cup\{i+1, j+1][i+1, j-1][i-1, j+1][i-1, j-1]\right\}
\end{aligned}
$$

Where $N_{4}$ and $N_{8}$ denotes 4-neighbour and 8-neighbour respectively. Therefore as defined by Jain et al (1995), given the binary image set $S$, a pixel $p \in S$ is connected to $q \in S$ if there is a consistent path of pixels between $p$ and $q$ consisting entirely of pixels of S. Jain et al (1995) noted that for any three pixels $p, q$, and $r$ in $S$, then connectivity properties exist such that:

1. Pixel $p$ is connected to $q$ (reflexivity).

2. If $p$ is connected to $q$, then $q$ is connected to $p$ (commutativity).

3. If $p$ is connected to $q$ and $q$ is connected to $r$, then $p$ is connected to $r$ (transitivity) 
Since objects have surfaces and points belonging to a surface are "spatially close", this technique can be used to label different regions of an image in order to identify the corresponding object of that image. One drawback with this method is that it can lead to bottlenecks in identifying image regions when there are many objects in the scene.

\subsection{Chapter Summary}

Vision research covers a broad domain with two principal areas, biological and synthetic vision research. Biological vision research deals with two main problems i.e. definition of the tasks involved in vision and formulating mechanisms that solves these tasks. This dual process of vision requires a basic understanding of both the physiological and mechanical characteristics of biological vision. The mechanisms relating to biological vision can be decomposed according to (Wandell 1995) into three key processes involving image formation, image representation and image interpretation. In this thesis, the decomposition of biological vision into its three components has provided a useful framework on which to discuss the structure of the vision model and its mechanisms. Synthetic vision research is concerned with developing computational models to represent these key processes and as such, several paradigms and algorithms have been developed.

It is also vital to define an approach for visual information processing mechanisms. These mechanisms are typically described as either top-down or bottom-up. Although in real life, both processes occur, each has its own advantages and disadvantages. A bottom-up view provides a hierarchical structure in which to segment visual information processing into task specific modules. On the other hand, a top-down view offers a more efficient approach to information processing since vision is directed to area of interest, only useful information pertaining to the specific task is processed. An understanding of visual information processing in humans provides a computational framework for developing perception mechanisms.

One of the key findings of the literature review is that although synthetic vision has been applied in many systems and virtual environments, within the area of driving simulation, visual perception is often either ignored or addressed at an abstract level. 
As such, each driver agent has perfect knowledge' concerning the positions and velocities of other vehicles in the simulation. Furthermore, vision is a complex process and visual perception modelling for autonomous virtual driver agents poses significant problems due to the dynamic nature of the traffic environment. However, it is possible to adapt some of the vision paradigms reported in ALife and AI systems to enhance autonomous driver agent behaviour within traffic/driving simulations. As pointed out by Thalmann (1995), synthetic vision "can be a powerful tool in modelling virtual autonomous actors in virtual worlds". This approach has many advantages and also introduces a contrast to traditional computer vision and digital image processing paradigms. For example, visual perception modelling for an ALife system is relatively fast and simple, and is used to support the emergence of complex behaviour within the overall system being modelled. The same can be expected through the introduction of vision modelling in driving simulation, providing new opportunities to investigate and mimic vision mediated cognitive processes such as object detection and recognition, collision avoidance, speed and distance perception within virtual driver agents.

Furthermore, the three main themes of this chapter have been biological vision, computer vision and ALife. Computer vision is a mechanistic approximation of biological vision. In the same way, ALife is also an approximation to biological vision, but is founded on principles that are more appropriate than many aspects of computer vision for simulation tasks. ALife offers a bridge between the performance of biological vision and the convenient mechanistic detail of computer vision.

In the context of driver simulation, the vision model in this thesis aims to deliver useful features of biological vision by using computer vision to support ALife. 


\section{CHAPTER 4}

\section{FUNCTIONAL DESIGN OF SYNTHETIC DRIVING SIMULATION}

\subsection{Introduction}

This chapter describes the conceptual framework of the Synthetic Driving SIMulation (SD-SIM). SD-SIM is a computer simulation environment for configuring traffic related parameters, simulation of these parameters and analysis of the simulation results. SD-SIM has three main components - synthetic traffic environment, vehicle dynamics and intelligent virtual driver. In this respect, SD-SIM makes a significant contribution to the current limitations of traffic simulation models by explicitly integrating the three main components in a well-structured and realistic manner. In addition to the overall discussion of the framework, this section focuses on the development of a vision model called SEE Scene En-capturing and Evaluation (Dumbuya and Wood (in press)) used within the virtual driver model (Dumbuya et al 2002b, Wood and Arnold 1997). In this respect, SEE supports intelligent decisionmaking, autonomous interaction between virtual drive agents, emergent traffic flow and overall behavioural realism within the simulation framework. Use of the vision model removes current limitations of virtual drivers having explicit access to the actual positions and velocities of other virtual drivers in traffic simulation (Dumbuya and Wood 2003). Instead, the vision model defines visual capabilities from individual virtual driver's point of view, allowing them to perceive their environment. Therefore, the functional characteristics of the vision model are similar to the synthetic vision paradigm proposed in (Noser et al 1995).

\subsection{Modelling Requirements and Specifications}

In addition to the requirements for realistic traffic simulation discussed in section 1.4.1, this section discusses the modelling requirements and specifications of SDSIM. These are summarised as follows: 
- Define, describe and develop mechanisms for driving simulation through extension of an existing driver decision-making model (Wood and Arnold 1997) and a vision model (Dumbuya 1999) using proven concepts and techniques.

- The framework should exploit concepts used in Artificial Life (ALife), Artificial Intelligence (AI) and Agent Based Simulation to model the inherent unpredictability and autonomous behaviour of drivers within traffic simulation models.

- Design an integrated framework in which driving simulation modules are represented in object-oriented techniques with opportunity for modularity, extensibility and maintainability of the different modules of SD-SIM.

- The framework functionality should demonstrate intelligent vision-mediated driver behaviour. Such behaviour should include (1) object detection and recognition (2) distance and speed perception (3) object tracking (4) collision avoidance (5) car following and (6) lane changing.

- Enable reconstruction and simulation of real-life driving scenarios based on gathered data.

- Conduct driving experiments to calibrate and validate the framework.

- The overall framework should contribute to the understanding of accident causation factors through evaluation of traffic scenarios, analyses of pre-crash influences and quantitative estimation of accident risk.

\subsection{Architecture of the Synthetic Driving Simulation Framework}

The diagram in figure 4.1 illustrates the conceptual architecture of the Synthetic Driving Simulation (SD-SIM) framework. The SD-SIM modelling framework and the incorporated visual perception model are both outputs from this research. SD-SIM also incorporates a previously developed driver decision making model (Wood and Arnold 1997). The framework consists of three main components: the Synthetic 
Traffic Environment (STE), the Vehicle Dynamics Model (VDM) and the Intelligent Virtual Driver (IVD). From a control system point of view the fundamental interactions within the framework can be described as either open-loop or closedloop. The open-loop operation is represented as a perception-decision-action sequence whilst closed-loop operation involves updating the driver/vehicle current state based on feedback from the synthetic traffic environment. Such feedback allows virtual driver agents to, for example, perform mental evaluation of the traffic situation so that any deviations from the desired goals could trigger corrective actions such as steering to avoid collision. The framework also attempts to replace some of the prescriptive nature of driving simulation models by adopting an agent-based approach that allows traffic to emerge as result of individual agent interactions.

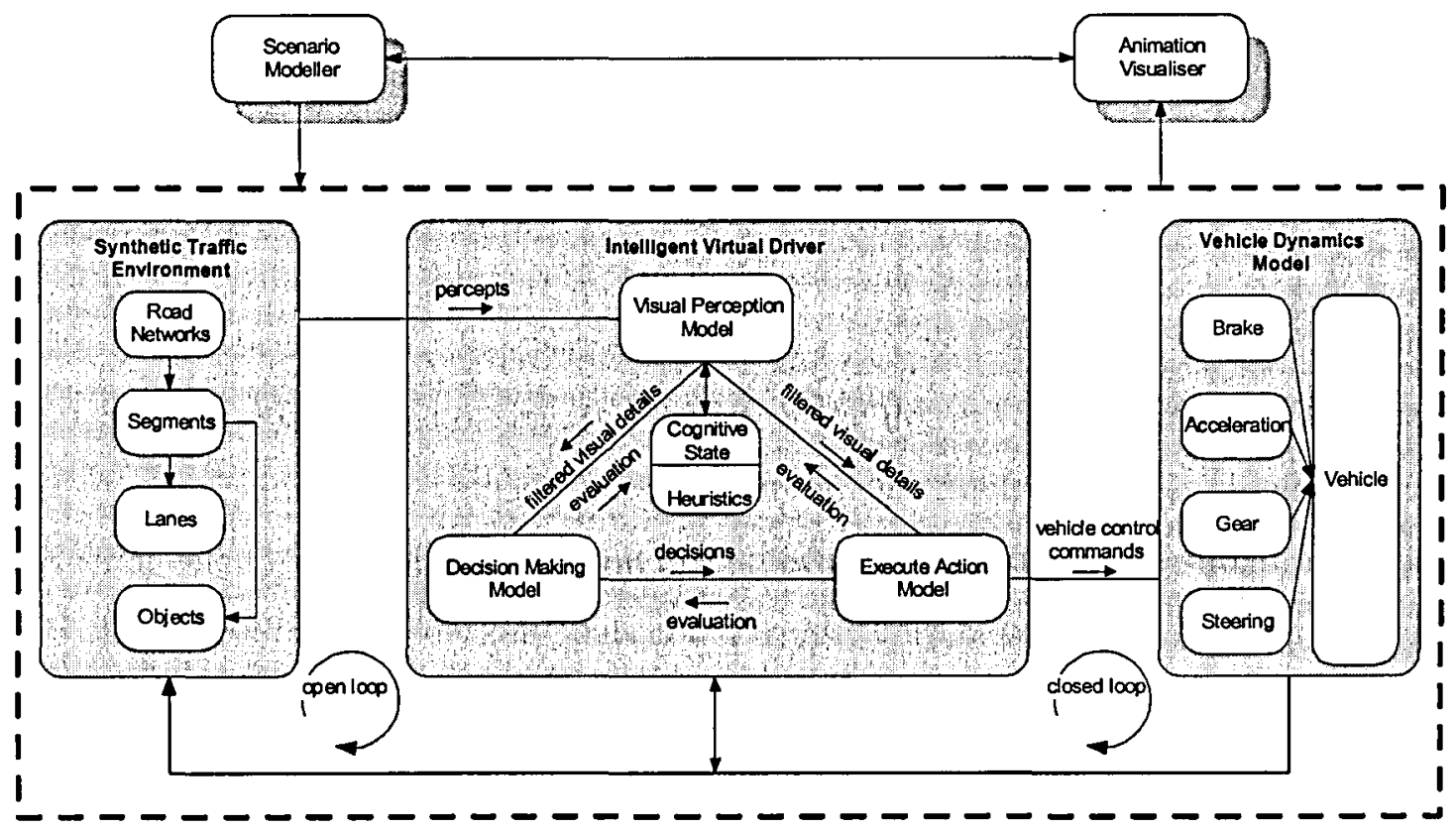

Figure 4.1: An agent-based approach to microscopic traffic modelling and simulation

A detailed description of the minimum inputs and outputs parameters that are handled in the framework are provided in Table 4.1. In configuring the IVD, the user needs to specify for example, position coordinates, plan coordinates, target coordinates, current speed, preferred speed, rules and vision parameters for each virtual driver agent. Typical output from the IVD include, time series data, identification of types if driver, current position coordinates, current lane number, direction, speed, distance and perception variables such as detected object, direction of motion, etc. 


\subsubsection{The Intelligent Virtual Driver}

The Intelligent Virtual Driver (IVD) module is the core of the SD-SIM and consists of three main subsystems; The Visual Perception Model (VPM), the Decision Making Model (DMM), and the Execute Action Model (EAM). These activities are performed by each driver agent, allowing individual driver agents to behave autonomously. The IVD module incorporates concepts from Artificial Life, Artificial Intelligence and Driver Psychology. Virtual drivers can be described as reactive (autonomous) agents because of their individual capability to perceive their environment, make decisions based on what they 'see' and take appropriate actions. This autonomous driver behaviour leads to the emergence of potentially complex and apparently unpredictable traffic flow due to interactions between individual agents. Figure 4.2 demonstrates the logical sequence of processes performed by a virtual driver agent within SD-SIM. More details of these processes are discussed in sections 4.3.1.1, 4.3.1.2 and 4.3.1.3 with section 4.4 describing the mechanisms of the visual perception model.

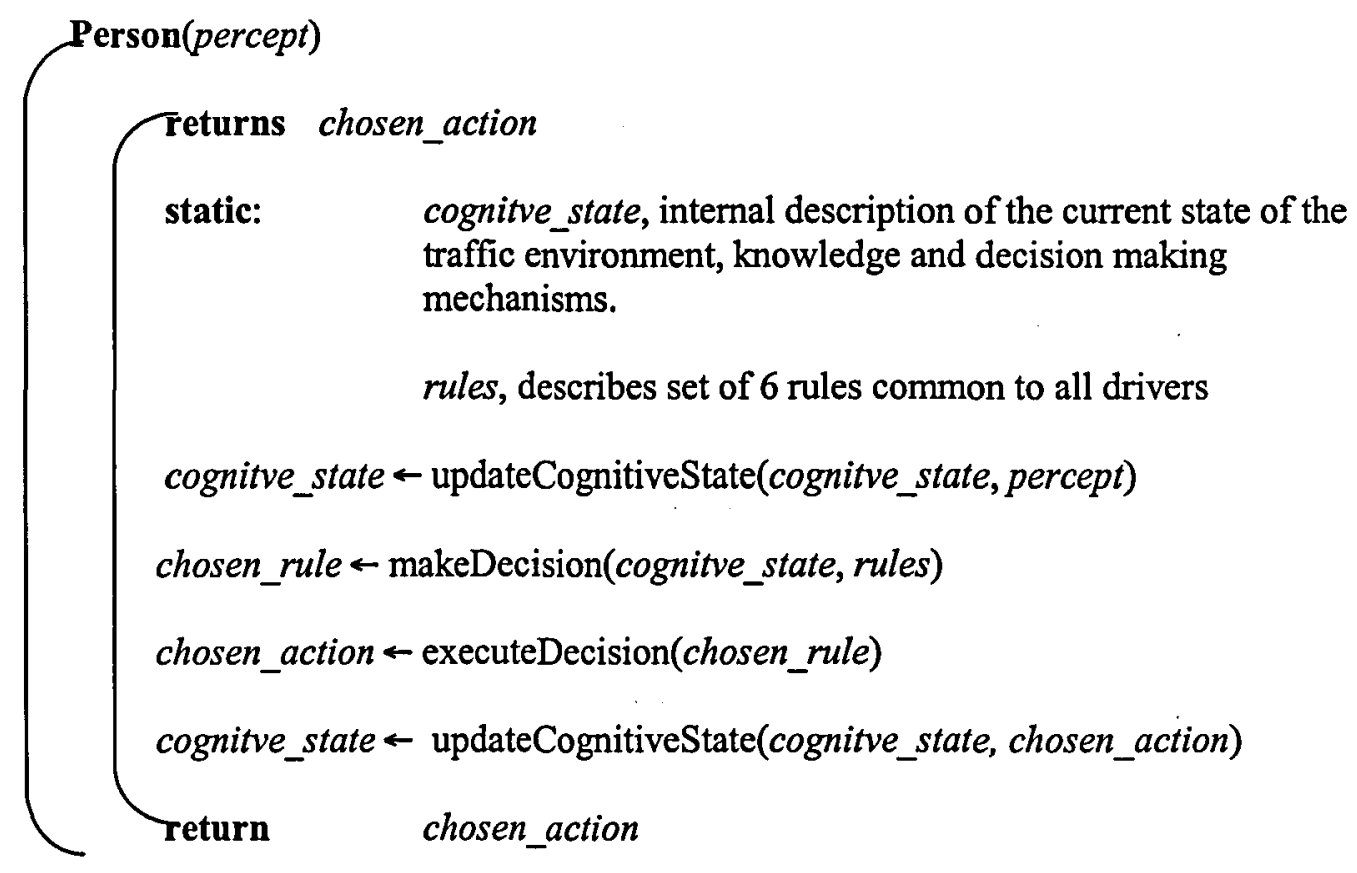

Figure 4.2: An agent-based implementation structure

\subsubsection{The Visual Perception Model (VPM)}

The VPM is based on the abstraction of key visual processes into a logical vision model. The agents' percepts are currently only associated with its vision capability 
but future implementations could include sound and body forces. Allied to this, a driver's cognitive state holds their knowledge of the current traffic environment. This knowledge representation is achieved in terms of visual information processing heuristics for object detection and recognition, estimation of apparent distance and speed, estimation of direction of motion, object tracking and collision detection. In this regard, SEE performs two important tasks: (1) Acting as a filter to exclude objects that are not within the visual field i.e. unsee-able objects and (2) introducing an element of uncertainty within driver decision making, since each agent must now perceive position and speed through estimation rather than using accurate data.

\subsubsection{The Decision Making Model (DMM)}

The $D M M$ describes the cognitive state of each driver agent and consists of driver preferences along with a set of rules common to all drivers. A detailed description of the decision making process is given in (Wood and Arnold 1997). Figure 4.3 illustrates the current six rules of the decision making process. Decisions are executed based on individual driver agents being able to use visual information received from the virtual traffic environment, determine the relevance of their rules and assess how important the relevant rules are. Although the rules are common to all drivers, each driver has a set of rule weightings, which are used to order the rules into descending importance of use. Driver agents' decision making also relies on each driver having a set of preferred parameters, which they aim to attain given opportunity within a simulation. These parameters include speed, distance headways (bumper to bumper distance between the lead vehicle and following vehicle) and lane number. The main factors preventing the preferred parameters from being achieved or forcing a driver to deviate from them is local traffic condition arising from other vehicles on the road. 


\begin{tabular}{|c|c|}
\hline Rule number & Rule description \\
\hline 1 & Slow down \\
\hline 2 & Speed up \\
\hline 3 & Maintain minimum headway \\
\hline 4 & Maintain minimum rear distance \\
\hline 5 & Maintain position within lane \\
\hline 6 & Change to preferred lane \\
\hline Visual Info \\
\hline
\end{tabular}

Figure 4.3 Co-operative decision making (Adapted from Wood \& Arnold 1997)

\section{Calculating Rule Relevance}

Each rule has to be relevant in order to be utilised and this relevance is calculated for each specific situation. Figure 4.4 for example demonstrates rule relevance calculations. The objective is to slow down. Therefore, if the perceived speed of the detected vehicle is equal to the driver's preferred speed, then the relevance calculation outputs 1 (yes) and the driver slows down. Otherwise, relevance calculation outputs 0 (no) and the relevance of the next rule is calculated. Similarly, the objective in rule 3 is to maintain minimum headway, so if the perceived forward distance of the detected vehicle is equal to or less than the drivers preferred forward distance, the relevance calculation outputs 1 (yes) and the driver continues to maintain a safe headway whilst following. Otherwise relevance calculation outputs 0 (no).
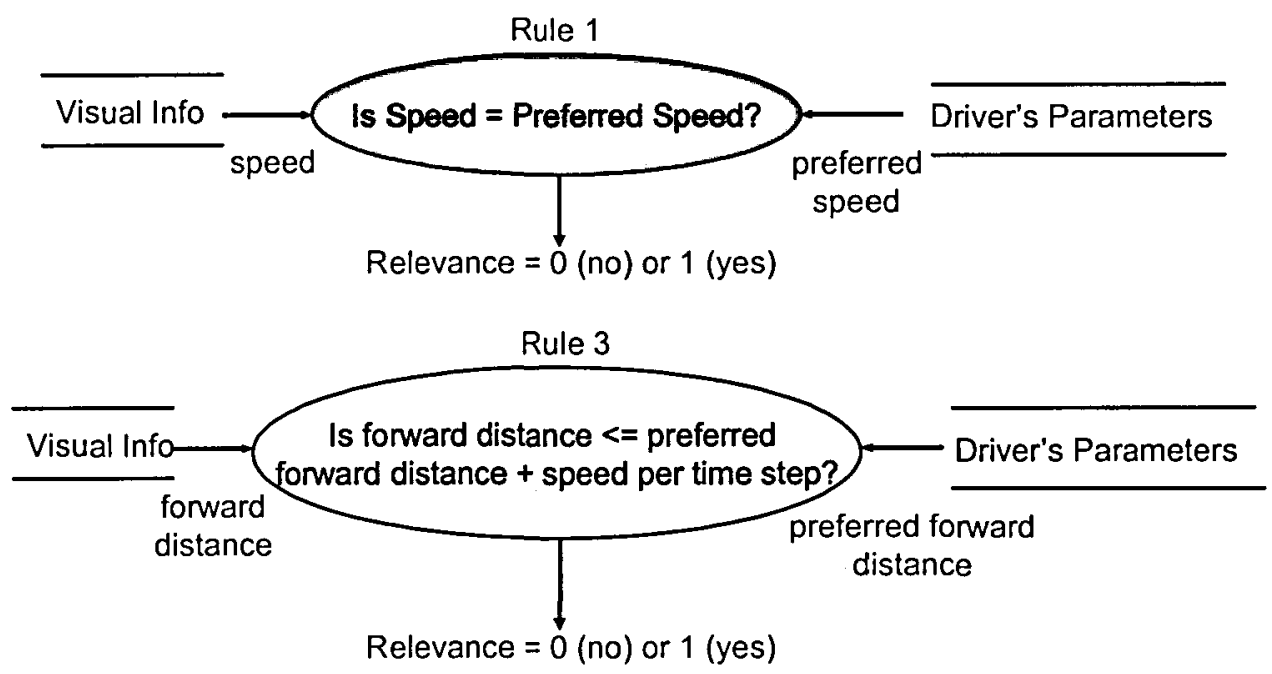

Figure 4.4 Example of rule relevance calculations 


\section{Determination of Rule Importance}

Rule importance, illustrated in figure 4.5 relies on each driver applying a weight value, which is between 0 and 1 to each of the 6 rules, where higher weight values imply greater importance. Such weights are specified prior to commencing the simulation, allowing each driver to be prescribed a rudimentary personality or driving style. For example, a relatively dangerous driver could be described by using a high weight for rule 2 (speed up), coupled with a low weight for rule 5 (maintain position within lane). The outcome of the decisions made by each driver is that the relevant rules are executed in order of decreasing importance. This results in each rule gaining a 'rule usage' value, calculated as the product of rule relevance, driver specific rule weighting and a random number factor. The random number factor allows for small degree of random behaviour and can be used (crudely) to reflect various levels of experience or expertise. The rule usage is calculated for all of a drivers rules and the most relevant value used. If it is not feasible that the rule with the highest rule usage value is executed first, then the second is used. This is known as the chosen rule and it leads to a course of action which involves either changing speed or steering.

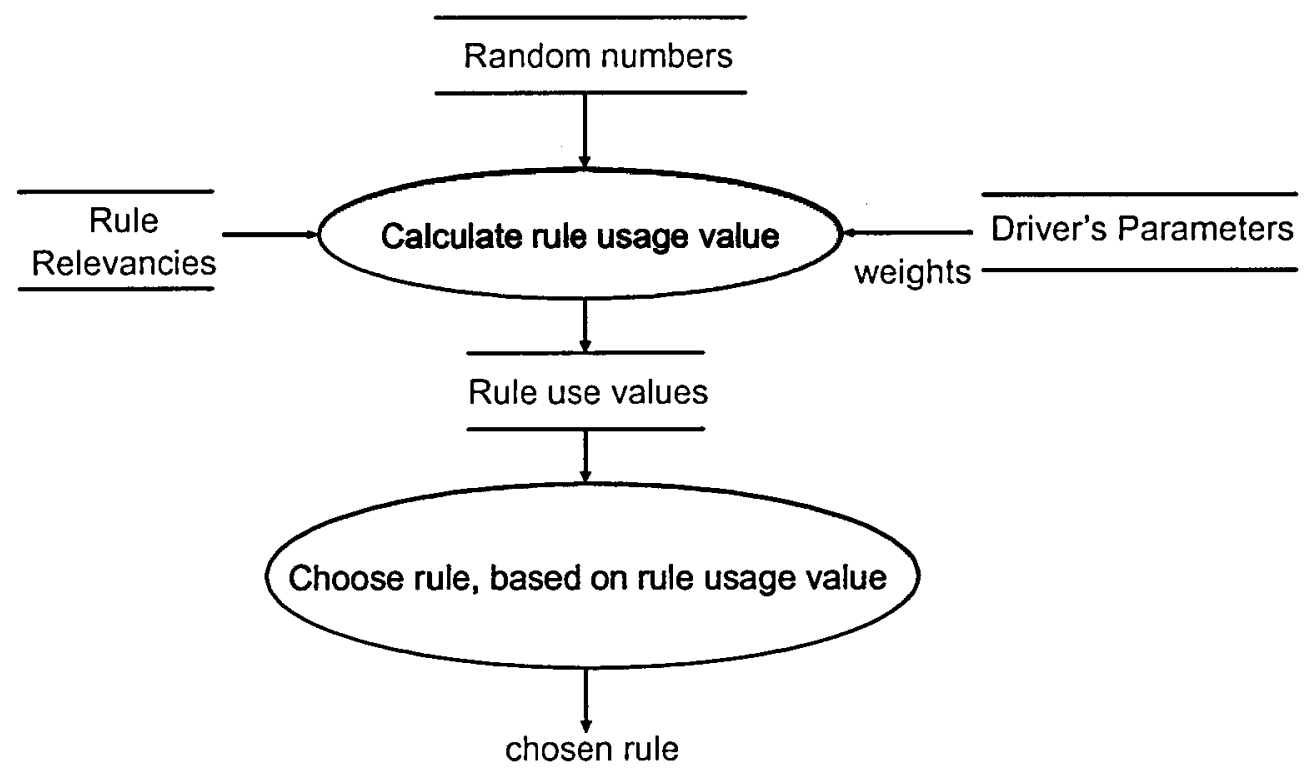

Figure 4.5 Example of rule relevance calculations 


\subsubsection{The Execute Action Model (EAM)}

The EAM component, part of the biomechanical system of the driver agent, performs control functions such as head gaze (e.g. scan within a wider field of view) and other driving related tasks. A complete 'man-model' derived from anthropometrics data is required to allow functionality of legs, hands and body of the driver agents. In this case, the man modelling approach in (Case et al 1990) or Thalmann's virtual humans (Musse and Thalmann 1997) will prove useful in representing and evaluating driver agents' responses to external forces. The execution of actions in terms of acceleration, braking and steering to effect vehicle control within the virtual environment is currently achieved by abstract processes. In other words the EAM currently represents the operational level of Michon's driver cognitive hierarchy (Michon 1985).

\subsubsection{The Vehicle Dynamics Model (VDM)}

The current implementation of the VDM module is primarily a container for the properties of a vehicle, e.g. dimensions, weight, selected gear and power available, with minimal functionality. Current parameters such as roll (centre of gravity to front and rear axles, height of mass, height of front and rear roll centres); suspension (suspension damping rate, front and rear stabiliser bar); gear-tyre model (gear ratio, coefficient of friction, cornering stiffness) can be defined within the framework. However, it is anticipated that as part of the On-The-Spot (OTS) project (Hill et al 2001), a more advanced vehicle dynamics model will be adopted. The features of such a model are described by Gordon in (Dumbuya et al 2002b).

\subsubsection{The Synthetic Traffic Environment (STE)}

In driving simulators, the visualisation of the virtual environment and believability of the generated traffic in terms of higher resolution, etc. is crucial. However, in traffic simulation, the focus is on the internal representation of the key components such as road network, vehicle, driver and their interaction, animation being a demonstration of these. The STE module - used interchangeably with Virtual Environment (VE), is responsible for creating the artificial environment for traffic. The STE consists of basic road geometry in terms of segments, lanes, road type, number of lanes, road 
gradients and simple logical information such as vehicles moving on the network and movement within and between lanes. The synthetic traffic environment structure can be described as non-deterministic because from the agents' point of view, they do not have direct access to the complete state of the traffic environment hence the agents' next state and actions are independent of the current state. At the same time the environment is discrete since the activities of the environment and the agent's actions are computed at fixed time increments. Furthermore, the driver/vehicle entity is connected to the road network by each driver/vehicle calculating its next target coordinates using the direction of the road, the vehicle current position and the direction the driver wishes to take.

\subsection{Visual Perception Modelling For Virtual Driver Agents}

The behaviour of traffic participants strongly depends on what they see and what they choose to do with the information gathered through seeing. Therefore, visual perception modelling is an important aspect of the virtual driver agents within SDSIM. However, vision is a complex process and visual perception modelling for virtual driver agents poses particular problems. The most simple yet most unrealistic approximation of driver vision in a microscopic simulation is to give each driver explicit access to the actual positions and velocities of other vehicles (Cameron et al 1994, van Wolffelaar and van Winsum 1995, Cremer et al 1996, Wright 1998). This amounts to each driver having 'perfect global vision'. Clearly, this is not realistic since human estimation of other vehicles' position, speed and direction depends on subjective interpretation of changes in the driver's local visual environment. The development of SEE is not intended to represent the complexity of human vision and as such, its underlying assumption is the abstraction of key visual processes into a simple logical model. This model is capable of low-level vision, through the use of simple visual information processing heuristics for object detection and recognition and estimation of objects position and speed. Therefore, in terms of concept the model is not too dissimilar to those formulated by (Noser et al 1995, Thalmann 1995, Tu 1999, Rabie and Terzopolous 2000). However, unlike these and other previous models (Yaeger 1994, Reynolds 1987, Blumberg 1997, Grand et al 1997), SEE is adapted to support intelligent decision making in driving with similarity to the traffic generation framework described in (Tran 1995), where interaction between individual 
agents is based on a perception-decision-action mechanism. This work differs from Tran's work in the way that individual virtual driver agents combine vision and decision-making capabilities within an ALife concept.

\subsubsection{Geometric Properties of the Visual Perception Model}

Figure 4.6a and $\mathrm{b}$ illustrates the concepts behind the Visual Perception Model. The visual capability of each virtual driver agent is constructed using simple vector analysis and ray tracing techniques. The vision model consists of two 'artificial eyes' each represented as a two-dimensional matrix consisting of $n$ rows and $m$ columns. Each eye is located on the front of a notional 'head'. A facet or cell figure 4.6a defines a single square or rectangle in the eye matrix so that for both $m$ and $n$ odd, each eye produces an outward normal at its centre. Imaginary visual rays are cast through facet centre points or pixels (figure 4.6a and $b$ ) into the virtual environment inhabited by vehicles which are currently represented as block primitives. For each eye all of the rays emanate from a focal point $\mathbf{R}$ referred to as the 'retina'. The word 'retina' is used here loosely to imply the analogue of light being focussed onto this point upon entering the eye. It also refers to the area of short term 'visual memory' for each eye, figure $4.6 \mathrm{~b}$, which contains several memory planes used for processing information in successive images. Individual memory planes specialise on specific visual functions or can be used to store differences between images. This acts as the driver's 'cognitive centre' where comparison is made between 'retinal' images and decisions. The point $\mathbf{H}$ in figure $4.6 \mathrm{~b}$ represents the centre of the head about which it can rotate and $\mathbf{a}_{1}, \mathbf{a}_{\mathrm{r}}$ are the respective left and right position vectors of the retinas. $\mathbf{R R}^{\prime}$ represents the base distance between the eyes measured from the outward normal of each eye. 


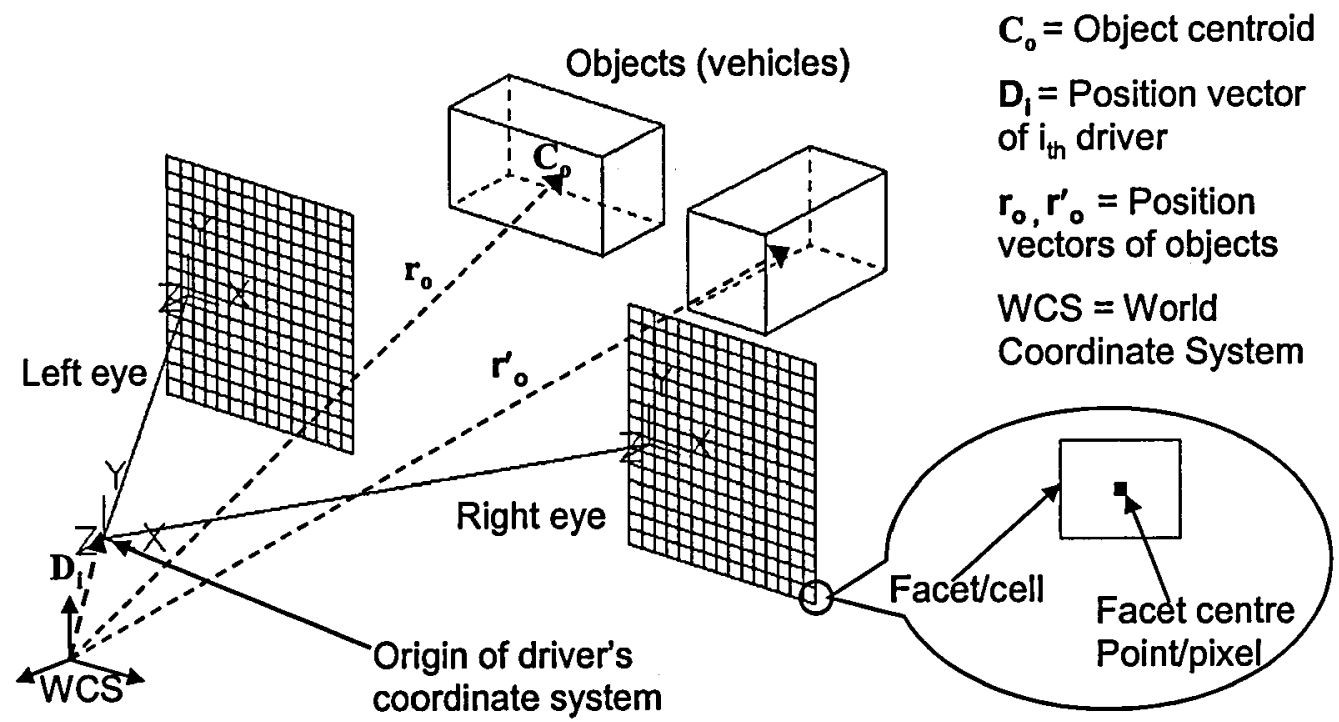

Figure 4.6a: Eye structure

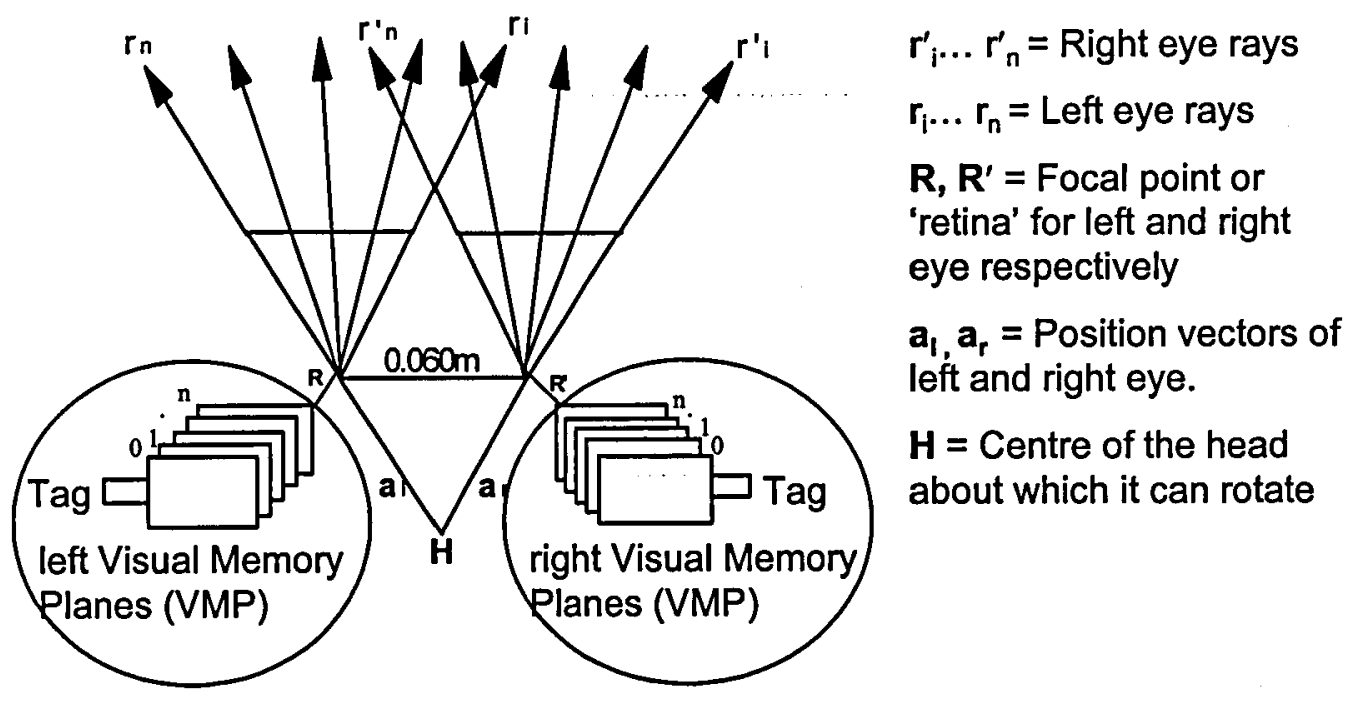

Figure 4.6b: Relationships between eyes, rays and memory planes

Most of the dimensional properties of the eye constructs are derived from human vision and the use of two eyes in the model help to achieve the estimation of simple stereo depths. It must be emphasised however that it is not the intention to build a vision model that exhibits the complexities of a biological vision system. The essential parameters and assumptions made during the construction of the vision model are: 
i. The maximum horizontal and vertical field of view (FOV) of each eye is $\theta=120^{\circ}$ and $\beta=150^{\circ}$ respectively.

ii. The focal point of the rays on the retina is $0.012 \mathrm{~m}$ behind each eye matrix.

iii. The horizontal and vertical dimension of each eye matrix is $0.024 \mathrm{~m}$.

iv. The $n m$ size of the eye matrix is an odd dimension i.e. both $n$ and $m$ are odd.

v. The base distance between each eye normal is $0.060 \mathrm{~m}$.

vi. The objects currently inhabiting the STE represent vehicles with dimensions defined by length, breadth and height and these values are supplied by the user.

vii. The vehicles are initially placed at state positions within the synthetic environment as part of the scenario definition.

viii. Eyes see or capture images at discrete times, time increments specified by the user.

ix. The right-hand convention is used throughout in defining the various co-ordinate systems.

The parameter values of $i, i i, i i$, and $v$ are typical of human vision system.

\subsubsection{The Image Capturing Process}

The image capturing process is relatively simple due to the geometric faceted approach, since the vision process does not rely on digital image processing techniques, which are computationally expensive. Using the vision model structure in figure 4.6, the images of objects within the synthetic driving environment are captured discretely from each driver agent's viewpoint as illustrated in figure 4.7, i.e. driver, $D_{i}$ in car, $C_{i}$ detects the presence of driver $D_{j}$ in car $C_{j}$. The drivers are located within the vehicle by the seat and head positions given respectively as $r_{\text {seat }}$ and $r_{\text {head }}$. The eyes, 
$r_{\text {eye }[i]}$ are relative to the origin of the driver's coordinate system, which is the centroid of the head, see figure $4.6 \mathrm{a}$. The height of the head relative to the body can be obtained from anthropometrics data and seat height can be measured directly.

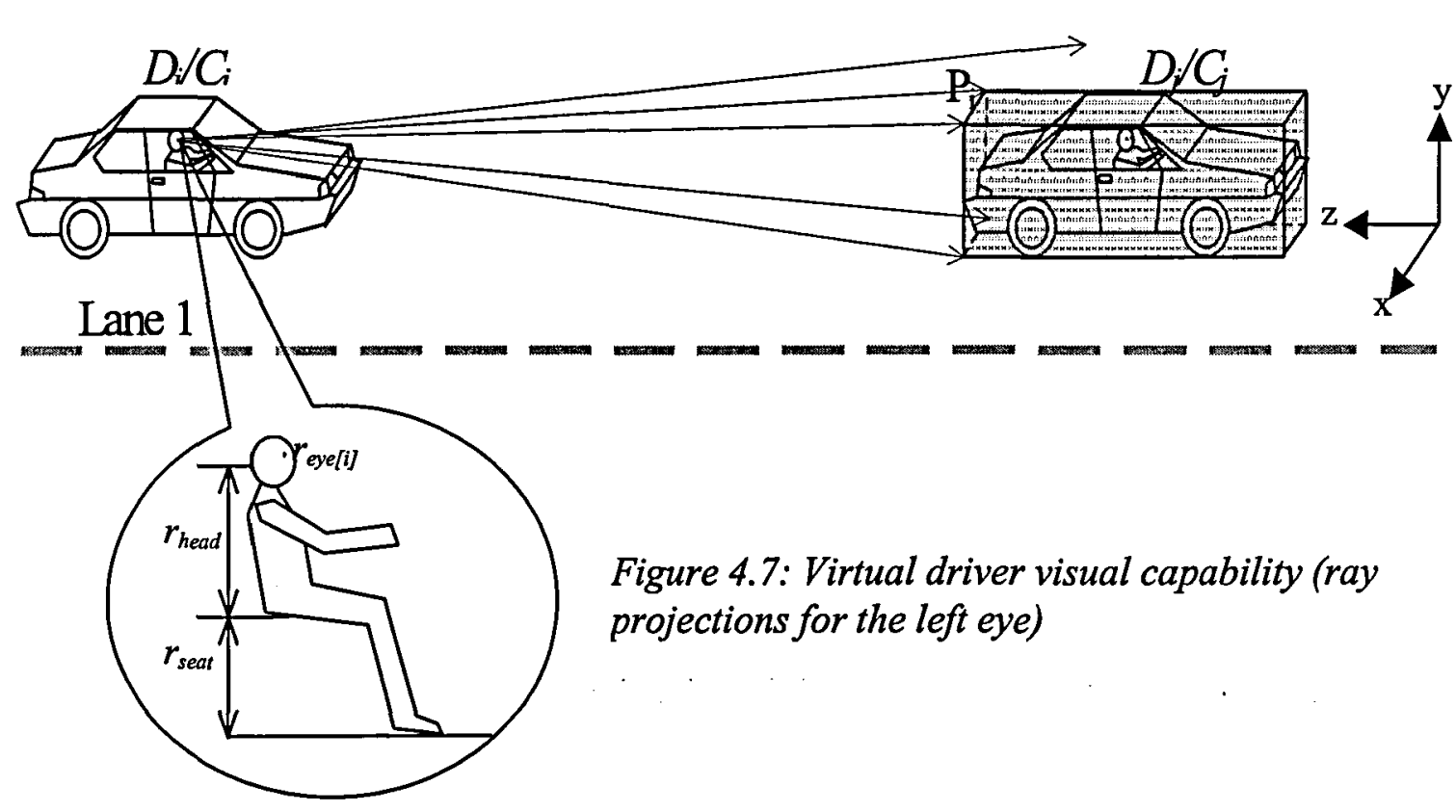

Each eye produces an array of rays cast through the centre points or pixels: $E_{a}^{\prime}=\left[r_{0}^{\prime}, r_{1}^{\prime} \ldots . . . r_{1+n}^{\prime} \ldots r_{n-1}^{i}\right]$ and each ray is defined by the general ray equation, $r^{i}=\mathbf{a}+\mathbf{b} t$. Where, $E_{a}{ }^{\prime}$ is the 'eye array' and the superscripts denoting either the left or right eye. Using the intersection of rays from the eyes and the object, the intersection parameter is determined according to equation 4.1 :

$$
t=\frac{D-n \bullet a}{n \bullet b}
$$

Where, $\mathrm{D}=\mathbf{n} \bullet \mathrm{P}_{\mathfrak{i}}$, is the plane data describing an object face. $\mathbf{n}$ is the face outward normal vector with components $\left(n_{1}, n_{2}, n_{3}\right) . \mathrm{P}_{\mathrm{i}}$ is a point with coordinates $\left(x_{i}, y_{i}, z_{i}\right)$ along each ray, measured from the 'retina' of each eye and $t$ is the intersection parameter of each ray on the object face. $\mathbf{a}$ and $\mathbf{b}$ are the position and direction vectors of each ray respectively. Therefore, each virtual driver agent has visual capability defined by the function, $D_{v}=V\left(x, y, Q_{u, d, l, r}, T\right)$ where $x$ and $y$ are the coordinates of the 
image captured in the driver's retina, $Q_{u, d, l, r}$ is the gaze angle describing gaze direction as up, down, left and right. $T$ is the time dimension at which images are captured or the frame rate. This time dimension is discretely sampled.

Intersection points on object faces are identified if the sum of angles between the intersection point on the object plane and the vertices on that plane following a clockwise sequence is approximately $360^{\circ}$. The intersection point is outside if the sum of these angles is approximately $0^{\circ}$. Currently, the eye facet through which the ray passes is assigned a binary value of 1 if the intersection lies inside the face boundary. Otherwise, it is assigned a binary 0 . This produces different 2D binary patterns of visible objects as a result of object movements and changes in orientation. This approach of image capture is similar to tactile sensing employed in robotics sensing technologies (Russell 1990, Cliff et al 1993) and also employed by many insects and animals using antenna and whiskers to navigate. It should also be noted that, with appropriate changes to the visual memory for each eye, colour perception will also be possible.

As in humans and insects, the ability to discriminate spatial patterns at higher resolution depends on the density of photoreceptors. Therefore an important parameter considered in the model is the 'eye resolution', by which the author means the ability to vary $n$ and $m$ for the eye matrices. This both increases the number of rays cast by each eye and reduces the inter-facet spacing so that greater detail can be detected. For example, a 9 by 9 eye matrix produces more rays that are closer together than a 3 by 3 eye matrix. The inter-facet spacing is defined as the distance between facet centre points (or pixels) and, hence, between rays emanating from the eyes. This concept is analogous to the mosaic effect used to improve visual acuity in faceted cornea of the compound eye of arthropodal organisms (Horridge 1975). However, the choice of eye resolution for the vision model should depend on the type of experiment and acceptable computational expense/time. The mathematical description of resolution starts with locating the outward normal ray as shown in equation 4.2.

For a given odd number of $n$ rows and $m$ columns, the outward normal ray produced in the centre of each eye is determined as shown in equation 4.2. 


$$
\begin{aligned}
& N_{t \rightarrow b}^{r}=N_{b \rightarrow t}^{r} \Rightarrow \frac{1}{2}(n-1) \\
& N_{l \rightarrow r}^{c}=N_{r \rightarrow b}^{c} \Rightarrow \frac{1}{2}(m-1)
\end{aligned}
$$

The terms $N_{t \rightarrow b}^{r}$ and $N_{b \rightarrow t}^{r}$ refers to the number of rows moved from top-to-bottom and bottom-to-top respectively to locate the outward normal in each eye and similarly, $N_{l \rightarrow r}^{c}$ and $N_{r \rightarrow b}^{c}$ is the number of columns moved from left-to-right and right-to-left respectively to locate outward normal in each eye.

Figure 4.8 summarises the details of the vision model in a generalised contents diagram. The figure shows the three major levels including image capture, visual information processing heuristics and vision mediated behaviour. Object detection performs image capture, tests for visibility of objects within the driver agent's field of view and determines the z-buffer. Image processing applies heuristics to analyse object motion and object recognition. These heuristics are implemented as algorithms for region labelling, calculations of centroid, bounding box, area, perimeter etc. The final level implements vision mediated behaviour such as distance and speed perception, etc. Some of the $\mathrm{C}+$ methods that implement these tasks are identified in the brackets.

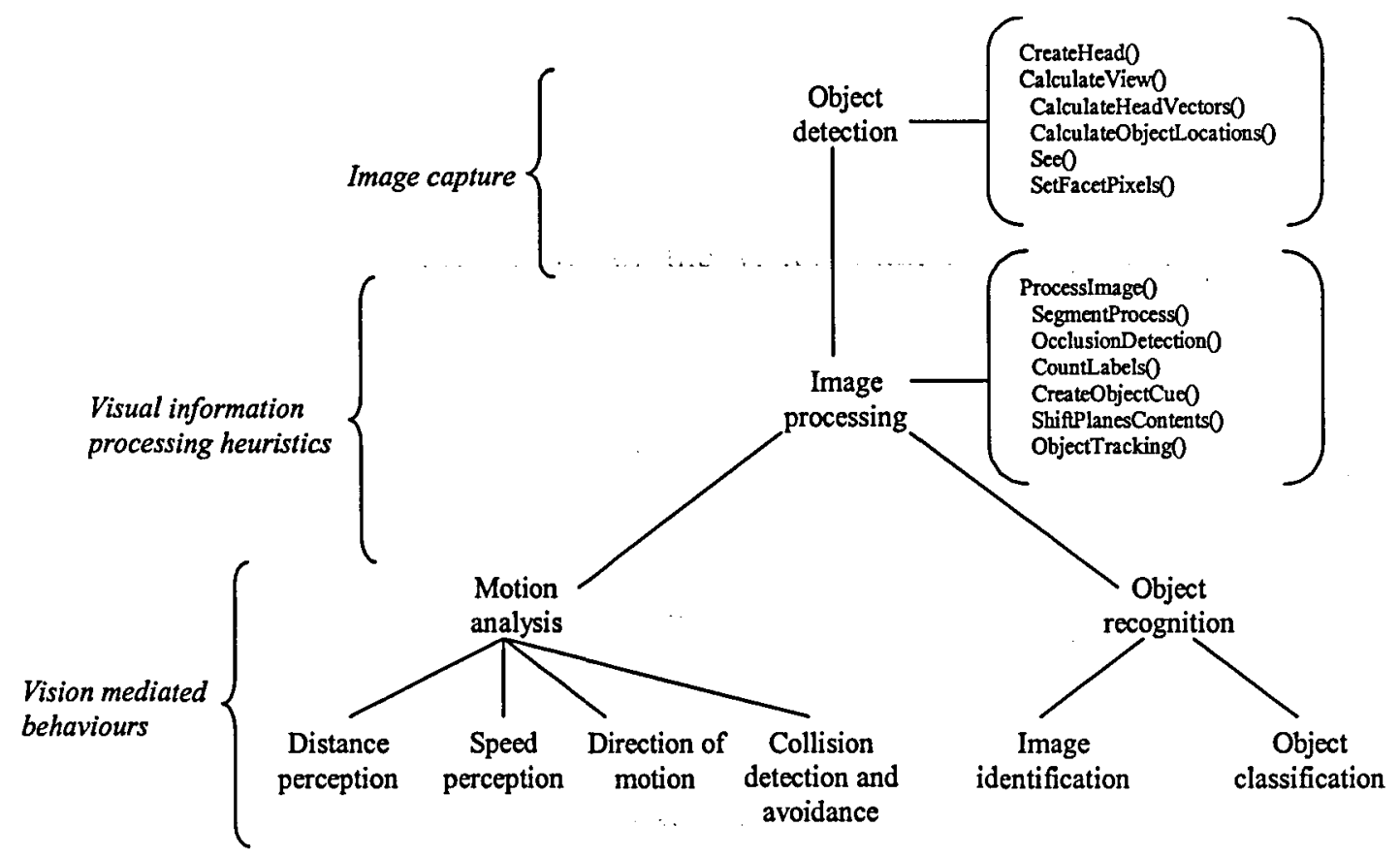


The remainder of this section considers the key mechanisms of image capturing and visual information processing within the model. The most important mechanism for image capture is the CalculateView 0 method. This method calls other methods such as CalculateHeadVectors $($ to determine the location of the eyes in relation to the head, CalculateObjectLocations 0 to compute the object normals, position and orientation of objects and See() to perform ray tracing to determine intersections between ray and object within the field of view. Details of the CalculateView() method are as follows:

\section{Initialise eye pixels to 0}

2. Call CalculateHeadVectors 0 method to compute position and direction vectors of the left and right eye relative to the head.

3. Call CalculateObjectLocations 0 method to calculate object normals, position and orientation

4. Call See0 method to acquire all objects within the defined field of view.

a. Loop for each eye, each facet row, each facet column, each object and each face of that object.

i. Calculate intersection point between the ray and object using equation 4.1

ii. Calculate the angle and sum of angles between the intersection point and points $P^{\prime}$ in the object plane.

iii. If the intersection between ray and angle $<120$ and $<150$ and sum of angles $\leq 360$ then,

- Object is visible within the field of view.

- Intersection is within boundaries of the object face.

- Set facet pixel value to 1 for the ray that intersects with the object face.

- Calculate intersection distance using Pythagoras Theorem.

iv. If intersection distance $>-0.05196 m$ (test for the location/position of the object in relation to the eyes i.e. distance must be in front of the eyes since negative direction of $z$-coordinate is in front and where $-0.05196 \mathrm{~m}$ is a fixed distance value calculated between retina and the origin of the driver's coordinate system) then,

- Object is in front of eyes.

- Calculate the smallest z-coordinates by applying z-buffer. 
v. If the calculated distance is less than the z-buffer then,

- Intersection point is nearer than last intersection point for facet.

- Update the facet information and only store nearer distance.

vi. Else, assign the z-buffer distance to the calculated distance and set relevant facet pixel value for each eye to 1 .

vii. If $b$ (iii) is false, then set relevant facet pixel value to 0.

b. Repeat calculations for all objects

5. Call SetFacetPixels method to build $2 D$ image map of each eye and pixel value at the specified location within each eye matrix.

\subsubsection{Computational Visual Information Processing Mechanisms}

This section describes the visual perception process in terms of image representation and interpretation. This is distinguished from the geometric image capture process using a cognitive description of perception that allows individual driver agents to maintain a distinct representation of their environment. Visual information processing by the virtual driver agents occurs in a notional 'retina' of the Functional Brain Model (FBM) shown in figure 4.9. The FBM integrates visual perception capabilities and actions of long-term memory. The model is currently biased towards visual perception and shows the three distinct processes described in (Wandell 1995), i.e. image formation, image representation (as part of retinal processing) and image interpretation. Image formation concerns the discrete capturing of images from the traffic environment into the left and right eyes of the virtual driver agents and was described in section 4.4.2. Image representation describes the pre-processing of images in the retina. This currently occurs in the Visual Memory Planes (VPM). In the VPM, some planes can alternatively be used to store differences between images. Retinal processing also supports driver behaviour by invoking actions such as head movements for driver agents to focus in a specific region of interest. For example lane-changing manoeuvre will require eye/head gaze to confirm adjacent lane is free and that any approaching vehicle from behind is at a sufficiently safe distance. 


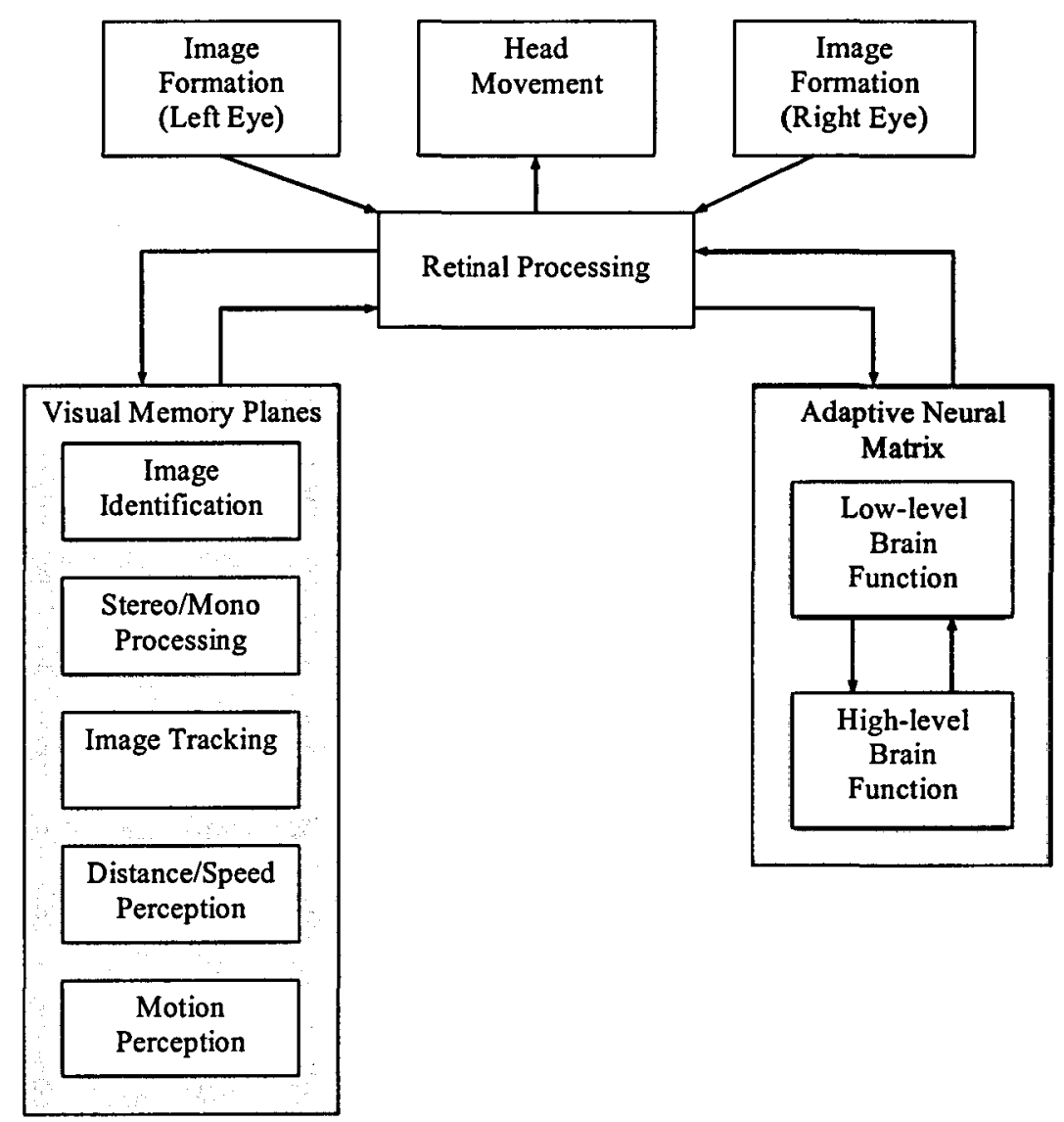

Figure 4.9: Functional components of visual information processing

The representation and interpretation of visual information in humans is very complex and involves many processes. The cognitive state of the FBM describes the internal operations of the driver agents in terms of visual information processing and decision making mechanisms. Both of these processes have been represented as heuristics to enable the virtual driver agents to respond to their environment. These heuristics are essentially AI algorithms intended to help simplify driver agent's analysis of complex traffic information. Algorithms have been adopted in line with (Crowley 1987), "most low-level perception and navigation tasks are algorithmic in nature; at the highest levels, decisions regarding which actions to perform are based on knowledge relevant to each situation". In addition, this method for representing visual information to support appropriate driving tasks and behaviour shares some similarity with Anderson's (Anderson 1996) production rules used to represent procedural knowledge for complex cognitive tasks. A discussion of the computational framework 
for visual information processing was presented in (Dumbuya and Wood 2002a) and a description of the driver's decision-making mechanisms also appeared in (Wood and Arnold 1997). The visual information processing heuristics are responsible for image identification, stereo/mono processing, image tracking, perception of distance and speed, and motion perception through processing binary images obtained from the image capturing process.

\subsubsection{Image Representation Heuristics}

The first task in analysing the images for representation purposes involves a regionlabelling algorithm to test for connectivity in the images so that unique image regions or patterns can be identified and described. Features (e.g. area, perimeter, centroid etc.) of each identified image region provide visual cues as to the objects. There are several region-labelling algorithms but a 4-connectivity region-labelling scheme for binary images similar to that in (Jain et al 1995) is implemented with modifications. This involves a two-pass strategy. The first pass scans left and right eye images labelling the binary values of the facets and building equivalence tables (to keep track of equivalent labels) at the same time. The second pass reprocesses the images updating the labels in the equivalence tables. From the identified image regions, the area, centroid and a bounding box of each region are determined. Details of this algorithm implemented in the Segment Process $[$ method is as follows:

1. Extract dimension information for the eyes/planes from the head.

2. Loop for each eye.

a. Scan image and label pixels, building equivalence tables.

b. Extract the cell values treating $x=0$ and $y=0$ as special cases for setting pixels to labels.

c. If only one of the pixel's upper and left neighbours has a label, then copy the label.

d. If both pixels have the same label, then copy the label.

e. If both pixels have different labels, then copy the upper label and enter the labels in the equivalence table as equivalent labels.

f. Otherwise assign a new label to this pixel and enter this label in the equivalence table.

3. If there are more pixels to consider, then go to step 2.

4. Find the lowest magnitude label for each equivalent set in the equivalence table. 
5. Reprocess image updating labels by replacing each label with the lowest label in its equivalent set.

Further processing to detect and remove occluded objects is required. Occlusion affects both object tracking and object recognition (discussed later). In object tracking, the presence of multiple objects poses difficulty in tracking them over a sequence of frames as the appearance and disappearance of objects in the frames could lead to some objects occluding others or being occluded. In detecting and removing occlusion, a standard computer graphics visibility test using scan line coherence and opening erosion techniques (Plastock and Kalley 1986; van Droogenbroeck 2002) are modified and applied in the OcclusionDetection ( method. The occlusion method tests the line of sight of other objects and for connectivity in the image regions. Partially occluded images are split by opening techniques and fully occluded objects are assumed to be single objects and therefore the algorithm does nothing.

1. Call ProcessImage0 method to determine visibility of objects and their depth

2. Determine if occlusion is likely i.e. which edges or surfaces are visible

a. Check whether the bounding boxes of the objects intersect (this uses the spatial coherence to assert that object whose bounding box don't intersect do not obscure each other. To do this first (1) determine the bounding box of the objects (2) check whether their bounding boxes intersect by comparing the corner points of the objects.

b. Using the vertices of the bounding box of object 1 and object 2 say, perform the following tests:

- Test 0: the $z$ extent of object 2 and object 1 do not overlap if zmax of object 2 is smaller than zmin of object 1

- Test 1: the y extent of object 2 and object 1 do not overlap

- Test 2: the x extent of object 2 and object 1 do not overlap

- Test 3: all the vertices of object 2 lie on that side of the plane containing object 2 which is farthest from the viewpoint

- Test 4: all the vertices of object 1 lie on that side of the plane containing object 2 which is closet to the viewpoint. 
c. Check if there are no discontinuities in image (pixel) connectivity (this uses the scan-line coherence to assert that if a pixel on a scan line lies within the polygon, pixels near it will most likely lie within the polygon). Hence if there are no discontinuities occlusion is likely. Check for the smallest $z$ value (depth) of the objects and determine whether the intersection depth $z_{1}$ of the first object is less than the intersection depth $z_{2}$ of the second object. If this is true, then once again occlusion between the two objects is likely.

d. For partially occluded objects remove by applying opening erosion algorithm (a fundamental mathematical morphology operation) to split the images. Alternatively, an edge detection algorithm could be employed. But an opening algorithm is demonstrated here.

- Loop through the first memory plane of each eye for all pixels $P(x, y)$.

- Take each binary image pixel (with value 1) with 4-connectivity

i. If $P(x, y)=0$, then do nothing

ii. If $P(x, y)=1$, and counter(label $(x, y),)<S$, then set $P(x, y)=0$. Where $S$ is a threshold using a binary structure element which is a matrix of ones. Note that the structuring matrix is hardwired in the implementation.

- Remove all regions smaller than S pixels.

e. For fully occluded objects

- Do nothing

A simple label and object association mechanism is employed in CountLabels() method, using the region labelling results to count the number of objects present in each eye's field of view. The details of this method are as follows:

1. Initialise the number of labels counter for all labels to 0

2. Loop through the first memory plane of each eye.

- Extract the $x$-and $y$-dimension information for the eyes/planes from the head.

- If $P(x, y)=0$, then do nothing

- If $P(x, y)=1$, then object found and increment the counter(label( $(x, y)$

- Measure the area of image region i.e. $A=\sum_{i=1}^{n} \sum_{j=1}^{m} B[i, j]$

- Calculate the mean distance of the region 
- Determine the bounding box of the image region

- Calculate the 2D image centroid $P(x, y)$ of the bounding box

The general principle of image capture is the same for stereo and mono vision in the model. However, the problem of stereo matching is inherently challenging. Although the vision model can estimate the distance at any intersection point from equation 4.1, the image capturing process does not extract intensities or edge information. Instead, each facet stores only two properties: a binary value of 1 or 0 indicating a hit or miss. In traditional computer/machine vision approaches, intensities are the main features extracted from digital images by active sensors. Here, the problem of stereo matching in its simplest form requires identification of a point in the left image that matches to a similar (generally in intensity) point in the right image. By estimating the disparities between corresponding pairs of points, the depth of the object projecting the two images can be determined. Many different approaches for solving this problem have been proposed over the years (e.g. Marr 1982). In the vision model however, the problem is one of matching image-to-object obtained from both eyes to ascertain whether both eyes are 'seeing' the same object. In other words, whether the left and right images are projections of the same object, but this is made more difficult by the fact that the images captured by the vision model do not exhibit intensity properties. It is necessary to match the correct pair of binary images to the correct object so only features relevant to that object are processed.

Figure 4.10 illustrates the vector analysis for the stereo process. First, calculate the mean distance of an image region due to all intersections inside an object face. Note the use of the word distance instead of depth. In his distinction of the two terms (Marr 1982) refers to distance as the 'objective physical distance from the viewer to the object measured from one of two eyes... and depth as the subjective distance to the object as perceived by the viewer'. This definition aptly applies to the vision model presented in this thesis. A unit vector is formed from the centroid of each image region in the left eye and then a 3D-centroid vector $3 \mathrm{D}-\mathrm{C}_{\mathrm{v}}$ (i.e. perceived object centroid) is projected into space by multiplying the unit vector by the mean distance. The 3D-centroid vectors are extrapolated by adding the co-ordinates of the position vector a. This is repeated for each image in each eye. Next, transform the position of 
the $3 \mathrm{D}-\mathrm{C}_{\mathrm{v}}$ (from the world co-ordinates) of the image region in the left eye to a $2 \mathrm{D}$ vector in the right eye called the $2 \mathrm{D}$ returned vector.

Similarly, a $3 \mathrm{D}-\mathrm{C}_{\mathrm{v}}$ is projected from the image region in the right eye to a $2 \mathrm{D}$ vector of the image region in left eye. If corresponding $2 \mathrm{D}$ vectors (i.e. their $\mathrm{x}$-and $\mathrm{y}$ - values) lie within the bounding box of the left and right eye image regions, then, it is conclusive that corresponding pair of image regions in the left and right eye are due to the projection of the same object in space. The apparent depth due to the effect of both eyes 'seeing' the same object is then calculated for all matched image-to-object pairs. The algorithm for matching image-to-object is formulated using basic principles of stereo geometry and follows simple vector analysis.

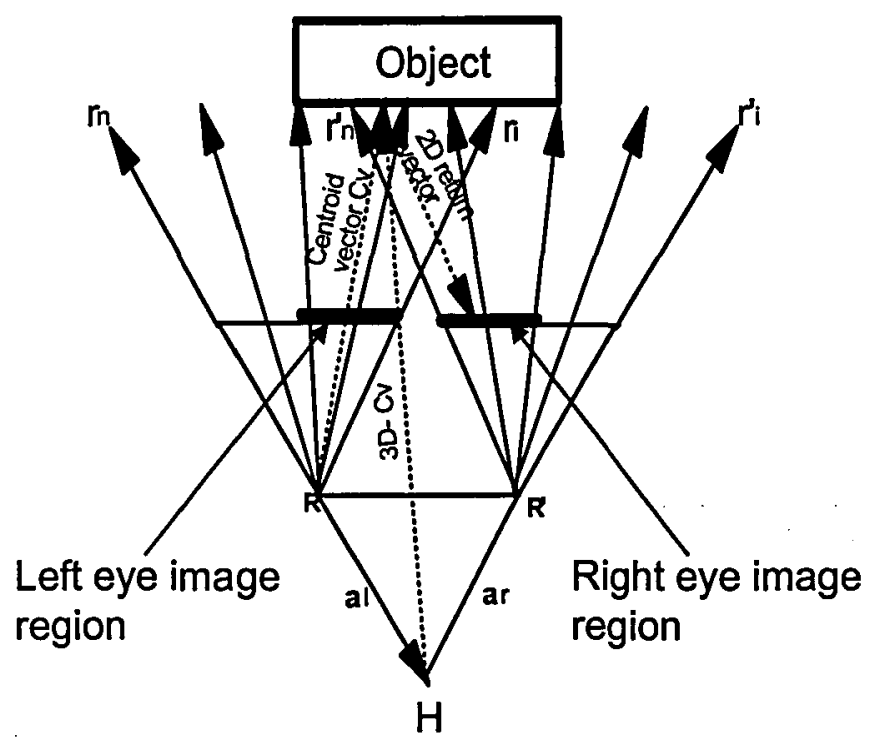

$r_{i}^{\prime} \ldots r_{n}^{\prime}=$ Right eye rays

$r_{i} \ldots r_{n}=$ Left eye rays

$\mathbf{R}, \mathbf{R}^{\prime}=$ Focal point or 'retina' for left and right eye respectively

$a_{1}, a_{r}=$ Position vectors of left and right eye.

$3 D-C_{v}=$ Perceived object centroid

$H=$ Centre of the head about which it can rotate

Figure 4.10: Vector analysis for the stereo process

Details of this algorithm implemented in the CreatObjectCue() method are as follows:

\section{Loop for each eye and loop for all identified image regions}

2. Check if $x$-object position is within stereo region of the field of view (assumption that eye matrices and hence image formed are coplanar, thus ignore vertical disparity i.e. $y=0$. Also stereo region is defined as half the base distance between each eye normal, which is $0.03 \mathrm{~m}$ and -0.03 for left and right eye respectively) If $(-0.03 m<=x<0.03 m)$, then object in stereo region 
a. Calculate mean distance, $\bar{D}$ of image region i.e. $\bar{D}=\frac{\sum_{i=1}^{1-n} P[i, j]}{A}$, where $P$ is a pixel at position $(i, j)$ and $A$ is the area of the image region.

b. Determine centroid, $(\bar{x}, \bar{y})$ of image region i.e. $\bar{x}=\frac{\sum_{i=1}^{n} \sum_{j=1}^{m} j P[i, j]}{A}, \bar{y}=\frac{\sum_{i=1}^{n} \sum_{j=1}^{m} i P[i, j]}{A}$

c. Construct a unit vector from the centroid of all $2 D$ images found in the left in the direction of the intersecting ray i.e. unit vector $=\frac{b i+b j+b k}{\sqrt{b i^{2}+b j^{2}+b k^{2}}}, b$ is the direction vector

d. Calculate and project a centroid vector of the $2 D$ image regions found in the left eye i.e. centroid vector, $C_{\nu}=\frac{b i+b j+b k}{\sqrt{b i^{2}+b j^{2}+b k^{2}}} \times \bar{D}$

e. Convert centroid vector into $3 D$ centroid vector of the image regions found in the left eye i.e. $3 D-C_{v}=\frac{b i+b j+b k}{\sqrt{b i^{2}+b j^{2}+b k^{2}}} \times \vec{D}+a$, where $a$ is the position vector

f. Transform $3 D-C_{\nu}$ object vector into $2 D$ returned vector in the right eye.

g. Repeat steps (a) to (f) for the right eye.

h. Match image found in the left and right eye

$i$. If the returned $2 D$ vector $x$-and $y$-coordinate values lies within the corresponding image region of each eye, then it is a stereo image.

ii. Associate stereo images to objects

iii. Calculate disparity map $\left(x_{l}-x_{r}\right)$ for each matched objects due the two eyes. Where $x_{l}, x_{r}=x$-displacement for the left and right eye at the point of intersection assuming head/eye are coplanar. Calculate the apparent depth $z$ due to this displacement, using $z=\frac{f d_{b}}{x_{1}-x_{r}}+a_{z k}$ where $f=$ focal length with value $0.012 m, d_{b}=$ base distance between the two eyes assigned value $0.060 \mathrm{~m}$ and $a_{z k}$ is the z-component of the position vector of the eye from retina to the origin of the driver's coordinate system with value 0.05196 (refer back to section 4.4.1). 
iv. Alternatively calculate location of the object in $3 D$ i.e. the midpoint of the disparity map relative to the head position due to all matched image-to-objects using $L_{\text {deph }}=\frac{\text { Centroid } 3 D[0]+\text { Centroid } 3 D[1]+\text { Centroid } 3 D[2]}{2}$

i. Else object is in peripheral field of view

i. If step (h. i) is false, then it is a mono image

ii. Associate mono images to objects found in the peripheral field

iii. Calculate the apparent distance of the mono object i.e. A $\alpha 1 / d$ using the zeroth order moment.

\subsubsection{Image Interpretation Heuristics}

Due to the dynamic nature of the vision process, a crude image tracking strategy is employed to decide whether an image region in each eye in the current frame is the same object represented by the same image region in subsequent frames. This task is particularly difficult when there are several objects moving independently in the environment and also when objects enter or leave the field of view. The strategy makes a common assumption, path coherence - (Jain et al 1995) that taking two consecutive frames at a time, there is only a small change in object movement between these frames, given a small enough frame time step. As illustrated in figure 4.11, the image tracking scheme is implemented using frame $n-l$ as a reference frame in which the default object data (displacement, labels associated with the objects, previous objects and object type) is initialised. The next step is to find an image in frame $n$ that is nearest to the current object in frame $n-1$ and calculate the smallest deviation $d$ between the frames. 


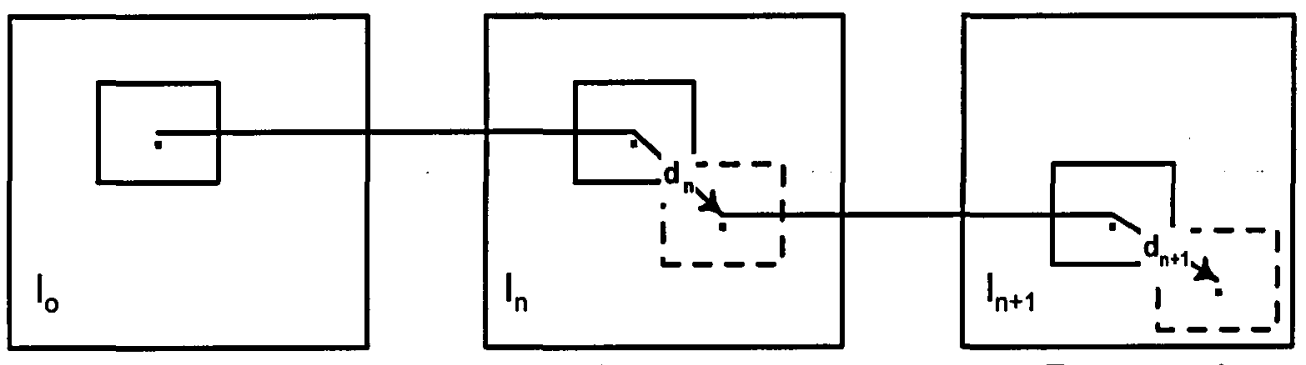

Frame $n-1$

Frame $n$

Frame $n+1$

$\mathrm{I}_{0}=$ Original image

$I_{n}=$ New image

$d_{n}, d_{n+1}=$ Smallest deviation between frames

Figure 4.11: Image tracking between frames

Applying the path coherence constraint, if the new image $I_{n}$ in frame $n$ has a small deviation $d$ relative to the old image $\mathrm{I}_{0}$, then the two images are the same. The constraint test is repeated for all subsequent frames e.g. frame $n+1$. The movement information for every matched object found in subsequent frames is updated and the frame sequences are used to compute object displacement. Details of this algorithm is as follows:

1. Take first two consecutive frames

2. Initialise frame n-1 as reference frame and set default object data

3. Loop for all objects in frame $n-1$

a. Check for occluded images, call OcclusionDetection 0 and remove.

b. Find object in frame $n$ closet to current object in frame $n-1$

c. Calculate object deviation between frames $n$ and $n-1$ using

$$
d_{n}=\sqrt{\left(x_{n}-x_{n-1}\right)^{2}+\left(y_{n}-y_{n-1}\right)^{2}+\left(z_{n}-z_{n-1}\right)^{2}}
$$

d. If calculated deviation, $d_{n}$ is less than a threshold value (1.e-30), or no previous object was found and matched, then
i. Object in current frame $n$ is the same as object in previous frame $n-1$
ii. Repeat steps $a, b, c$ for all objects in subsequent frames. 


\section{For all tracked objects within the frames, update movement information}

a. Calculate displacement of each tracked image/object

A further decomposition of the image processing mechanisms leads to vision mediated behaviour involving motion analysis and object recognition. Motion analysis is critical since human perception in many situations is more sensitive to moving objects than stationary ones. The various tasks involved in motion analysis are demonstrated in figure 4.8. Currently the process of estimating apparent speed and distance is achieved through the use of a 16-plane linked list structure, with visual memory planes in each eye responsible for storing consecutive image sequences, figure 4.6b. Each memory plane is associated with a tag used to extract encoded information from the planes so that specific visual functions can be performed. This introduces realistic perceptual uncertainty by forcing individual driver agents to make a subjective interpretation of changes in their field of view, rather than rely on global information. For example, as part of the image tracking algorithm, apparent speed is estimated in the visual memory planes by simply dividing the displacement length between frames over the frame time step.

Evaluation of direction of motion is illustrated in figure 4.12. Since the area cast on the eye retina by a detected object is inversely proportional to the distance of the object i.e. $A \propto 1 / d$, apparent direction of motion can be inferred from either looming (L) or receding (R) effect by testing the sign of the z-coefficients of the displacement vector (see figure 4.6) i.e. the distance. For example, if the area or shadow cast is growing and the distance is reducing, then it is possible that the detected object is looming or driver agent is approaching a stationary object. If on the other hand, the area cast is growing or shrinking and the distance is constant, the detected object maybe stationary with a different orientation. This way of describing motion is useful in providing visual cues of the state and position of objects. Various other cognitive processes e.g. focus of attention, anticipation or collision avoidance, associated with driving can also be achieved by comparing image patterns between consecutive memory planes. For example, any sudden enlargement of a pattern within an image could trigger appropriate anticipation measures including for example, focus of 
attention to warn the driver of the impending rear collision with a leading vehicle due to approaching closer than the safe driving distance.
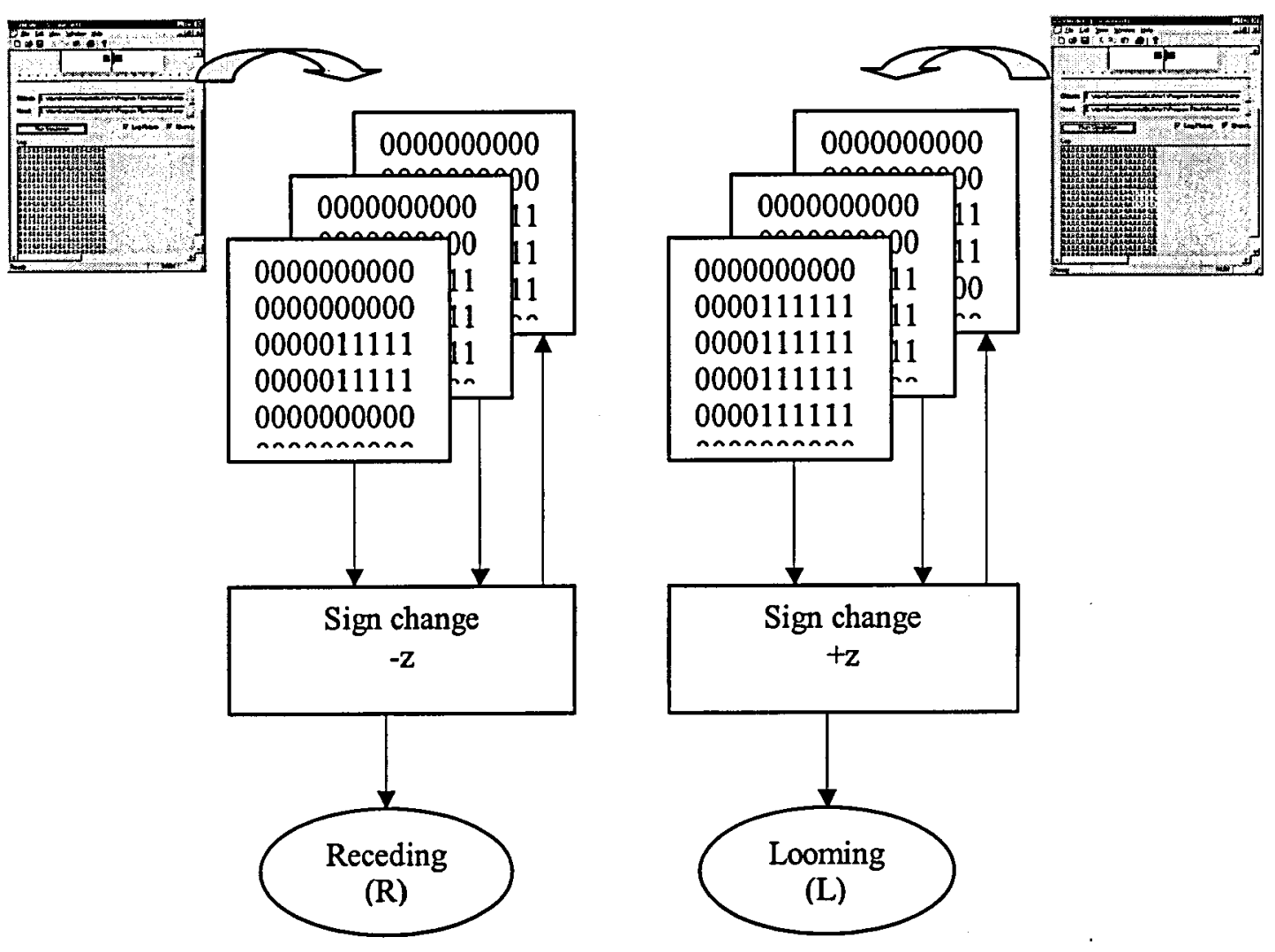

Figure 4.12: Motion analysis for looming and receding heuristics

In algorithmic form, the motion perception heuristic is

1. Loop for each eye

2. Compute area and depth of tracked images in frame $n$ and frame $n-1$

a. Take the first two consecutive frames (frame $n$ and frame $n-1)$ of the visual memory planes

b. If area, $A$, cast on the eye is expanding and depth $L_{\text {depth }}$ is reducing (i.e. change in sign of the z-coordinate of the depth vector due to a horizontal image shift from frame $n$ to frame $n$ 1), then the object is looming

c. If area cast on the eye is expanding or decreasing and depth is unchanged, then object is stationary with change in orientation 
d. Else if $b$ and $c$ are false, then object is receding.

3. Save direction of motion information in another visual memory plane

4. Repeat for all objects detected

As objects move within the synthetic environment, there is continuous potential for collision with each other. Looming and receding effects can provide some information as to any impending collisions, but driver agents must also be able to predict their nearest approach with other driver agents to evaluate threats of collision. Details of the algorithm evaluating collision detection and avoidance is given next. The algorithm applies rules from (Wood and Arnold 1997), listed in section 4.3.1.2

\section{Check if looming object poses a collision threat}

a. Calculate interspacing distance, $z$ between current driver and looming objects (this is essentially the z-buffer distance obtained from rendering the image using the centroid of the bounding boxes of both current driver and detected or looming object).

b. If looming object and interspacing distance $z$ are within an unsafe threshold value (or the proximity of the nearest object), then collision is eminent.

c. Else, no threat of collision.

2. If 1.6 evaluates to true, then apply corrective action (or collision avoidance) using decision mechanisms.

a. If change speed, then apply rules 1, 2, 3, 4, driver's preferred parameters and check lane change.

b. If check lane change, then apply rules 6, 3, 4 and driver's preferred parameters.

c. If steer, then apply rule 5 and lane change.

d. If move, then apply speed change and direction change.

3. If $1 b$ evaluates to false or $1 c$ is true, then continue to track object at each time step until object is no longer within field of view.

Finally, object recognition requires image identification (in the segmentation process of SegmentProcess() method, section 4.4.3.1) and classification. The classification model involves comparing the features of an unknown image against known 
characteristics of vehicles. The relative size of vehicles is used to distinguish the various types of vehicles by measuring the area of each distinct region and comparing it to known characteristics of vehicles.in terms of their sizes. For example cars and lorries are distinguished simply by their relative dimensions. Therefore the driver agents utilise cognitive models - internal representation of the knowledge of vehicle dimensions to determine the type of vehicle detected in each time step. The classification scheme currently employs the aspect ratio of objects by comparing similar properties such as length, width and height against internal representation of vehicle properties. The cognitive and computational processes involved are simple but sufficient to categorise the type of vehicles detected into cars and lorries. However, this approach is limited in that the selection of attributes for comparison is rigid and also in real driving situations human driver's judgments are very complex. As a result new research is being identified to develop a more realistic low-level brain function called Adaptive Neural Matrix (ANM) model (see figure 4.9) to emulate the neuronal mechanisms of vision and perception. Details of the current object recognition algorithm are as follows:

\section{Determine aspect ratio of car and lorry}

2. For all detected object

a. Record image dimensions

b. Calculate image aspect ratio

c. Compare aspect ratios

i. if(abs((int) (Lorry_Ratio-Object_Ratio) $<$ abs((int) $($ Car_Ratio - Object_Ratio))), then image represents a lorry

ii. Else, image is classified as car

Figure 4.13 summaries the different activities of the visual perception algorithm, showing the flow of control between activities. The process starts as part of the user's scenario modelling by the specification of head parameters (e.g. eye resolution, frame rate etc.) for each virtual driver agent. As the simulation is instantiated, the scene (containing traffic participants) is loaded and the view from each agent is interpolated between the frames. During the interpolation, each driver's view is calculated and the 2D image patterns are shifted to the visual memory planes where further cognitive 
processing is carried out to determine object detection and recognition, distance and speed estimation, direction of motion etc. In addition, the frame rate of the vision model i.e. the rate at which driver agents perceive their environment is currently the same as the overall simulation update rate.

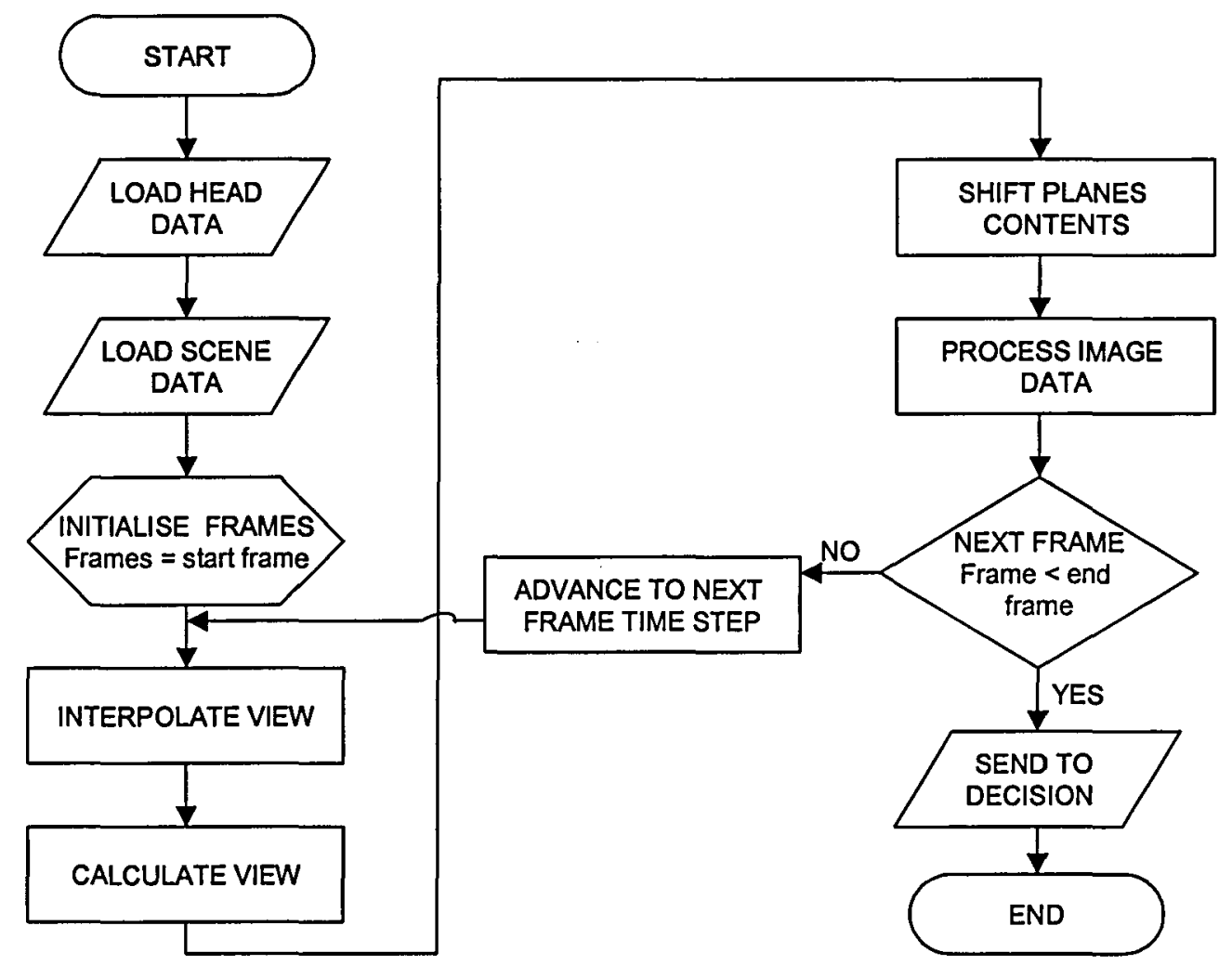

Figure 4.13: Activity of the visual perception model

\subsection{An Integrated Simulation Framework}

This section describes the design of the integrated simulation framework of SD-SIM. The intention of the design methodology is to reflect both the requirements and specification objectives set out in section 4.2. The formal approach adopted involves the standard Unified Modelling Language (UML) notation, version 1.3 (The Object Management Group). This approach is consistent with the principles of agent abstractions, which are based on traditional object-oriented design methodologies (Shoham 1993, Bergenti and Poggi 2000) rather than any specific agent-based software implementation paradigm. As such, it offers an effective approach to representing structure, behaviour and associated complexities in both real world and software systems. In addition, the main design considerations of the integrated 
framework are summarised in table 4.1. The input and output parameters defined in this table are not exhaustive but illustrate the type of data that is handled in the framework. Section 4.5.1 identifies the system domain and interactions with the user of the system.

\begin{tabular}{|c|c|}
\hline \multicolumn{2}{|c|}{ Intelligent Virtual Driver $\quad$ Functional Description } \\
\hline Model type & Person, currently defined as driver \\
\hline Level of detail & Microscopic \\
\hline Perceptual processes & $\begin{array}{l}\text { Vision model used to support object detection and } \\
\text { recognition, distance, speed and motion perception }\end{array}$ \\
\hline Cognitive processes & Knowledge, Decision making capabilities \\
\hline Control functions & $\begin{array}{l}\text { Abstracted with rule combinations to define driver } \\
\text { personalities and behaviour }\end{array}$ \\
\hline Driver behaviour models & $\begin{array}{l}\text { Car following, overtaking, are not explicitly modelled } \\
\text { but emerged as a consequence of interactions }\end{array}$ \\
\hline Input (initial parameters) & $\begin{array}{l}\text { Position coordinates, plan coordinates, target } \\
\text { coordinates (heading), current speed, preferred speed, } \\
\text { rules, vision parameters }\end{array}$ \\
\hline Output & $\begin{array}{l}\text { Time, driver identification, current position } \\
\text { coordinates, current lane number, direction, speed, } \\
\text { distance, perception variables. }\end{array}$ \\
\hline \multicolumn{2}{|c|}{ Vehicle Dynamics Models $\quad$ Functional Description } \\
\hline Model type & Vehicle currently defined as car \\
\hline Level of details & Rudimentary and minimal functionality. \\
\hline Static properties & Vehicle dimensions, mass are considered \\
\hline Kinematic properties & $\begin{array}{l}\text { Position, motion, velocity, acceleration, steering, } \\
\text { braking and planar 2D motion are available }\end{array}$ \\
\hline Dynamic properties & $\begin{array}{l}\text { Basic forces, like friction, rolling, suspension are } \\
\text { defined but doesn't affect vehicle behaviour }\end{array}$ \\
\hline Input (initial parameters) & $\begin{array}{l}\text { Position coordinates, dimensions, weight, maximum } \\
\text { power, maximum speed and maximum acceleration etc. }\end{array}$ \\
\hline Output & $\begin{array}{l}\text { Vehicle identification, current position coordinates, } \\
\text { speed, acceleration, distance covered, }\end{array}$ \\
\hline \multicolumn{2}{|c|}{$\begin{array}{l}\text { Synthetic Traffic Environment } \\
\text { Functional Description }\end{array}$} \\
\hline Model type & Road network defined as a collection of segments \\
\hline Level of details & Low-level computer generated rendered scenery \\
\hline Functional design & Traffic flow \& network performance not considered \\
\hline Geometric design & $\begin{array}{l}\text { Simply road geometry represented as motorway. } \\
\text { Features include straight segments, lanes. }\end{array}$ \\
\hline Structural design & Not considered \\
\hline Logical information & Scenario data containing lane width, traffic rules etc. \\
\hline Input (initial parameters) & $\begin{array}{l}\text { Segment type, number of segments, number of lanes, } \\
\text { lane width, road direction, total length of road, street } \\
\text { furniture added from the database. }\end{array}$ \\
\hline Output & Rendered view \\
\hline
\end{tabular}




\subsubsection{System Interactions}

To foster reusability and maintainability within the several interacting components of SD-SIM, a higher-level design view of the integrated system has been taken. In this respect, three packages containing the VPM classes, IVD classes and Graphical User Interface (GUI) classes are identified in figure 4.14. It is important to note that although the vision model is conceptually an integral part of the driver model, for the purpose of design it is beneficial to separate the two. Furthermore, since the two models were developed separately, it is also vital to maintain this modularity in order to be able to add functionality into either model in future developments.

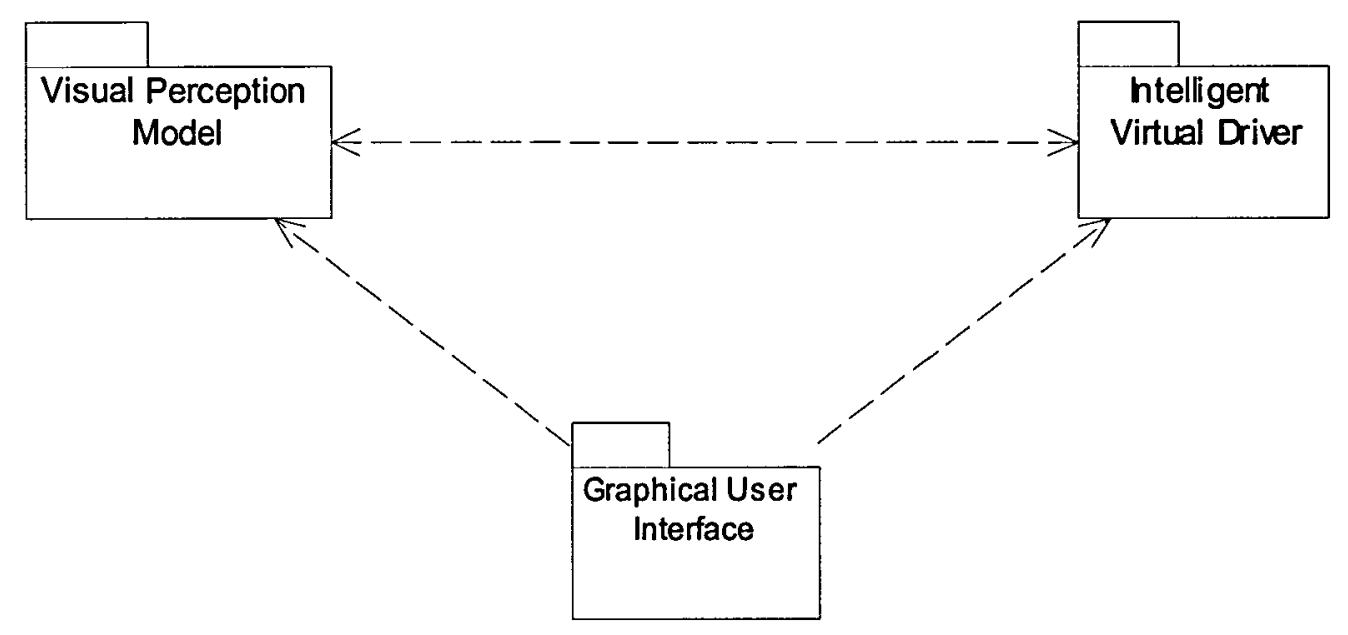

Figure 4.14: SD-SIM System packages

Each package in figure 4.14 depends on the other, with a bi-directional association or information flow between the VPM and IVD. Typical information flow between these models includes head information containing percepts, detected objects, depth and motion information, etc. The GUI requires information from both the VPM and IVD models to assemble the data for presentation to the user. Taking a lower-level view of figure 4.14, i.e. looking at the contents of the packages, figures 4.15 summarises the generic conceptualisation of the integrated simulation framework in terms of the class interaction within the packages. The implementation of SD-SIM based on instances of these classes supports the creation and manipulation of traffic simulation entities. Appendix B.1 provides a more detailed description of the system showing the attributes and operations of the various classes within the system. 
The main features depicted in figure 4.15 are inheritance, aggregations and unambiguous associations. For example, Driver and Car classes inherit from the Person, and Vehicle classes respectively. This implies that the attributes and functionalities of Person and Vehicle classes are also available to Driver and Car, with the possibility of further definition of attributes and functionalities within these subclasses. Aggregation is demonstrated for example, between RoadNetwork, Segment and Intersections, in which a RoadNetwork class is made up of Segment and Intersections. Furthermore, unambiguous associations are denoted by semantic relations such as needs, makes, activates, used_in, part_of and drive_on.

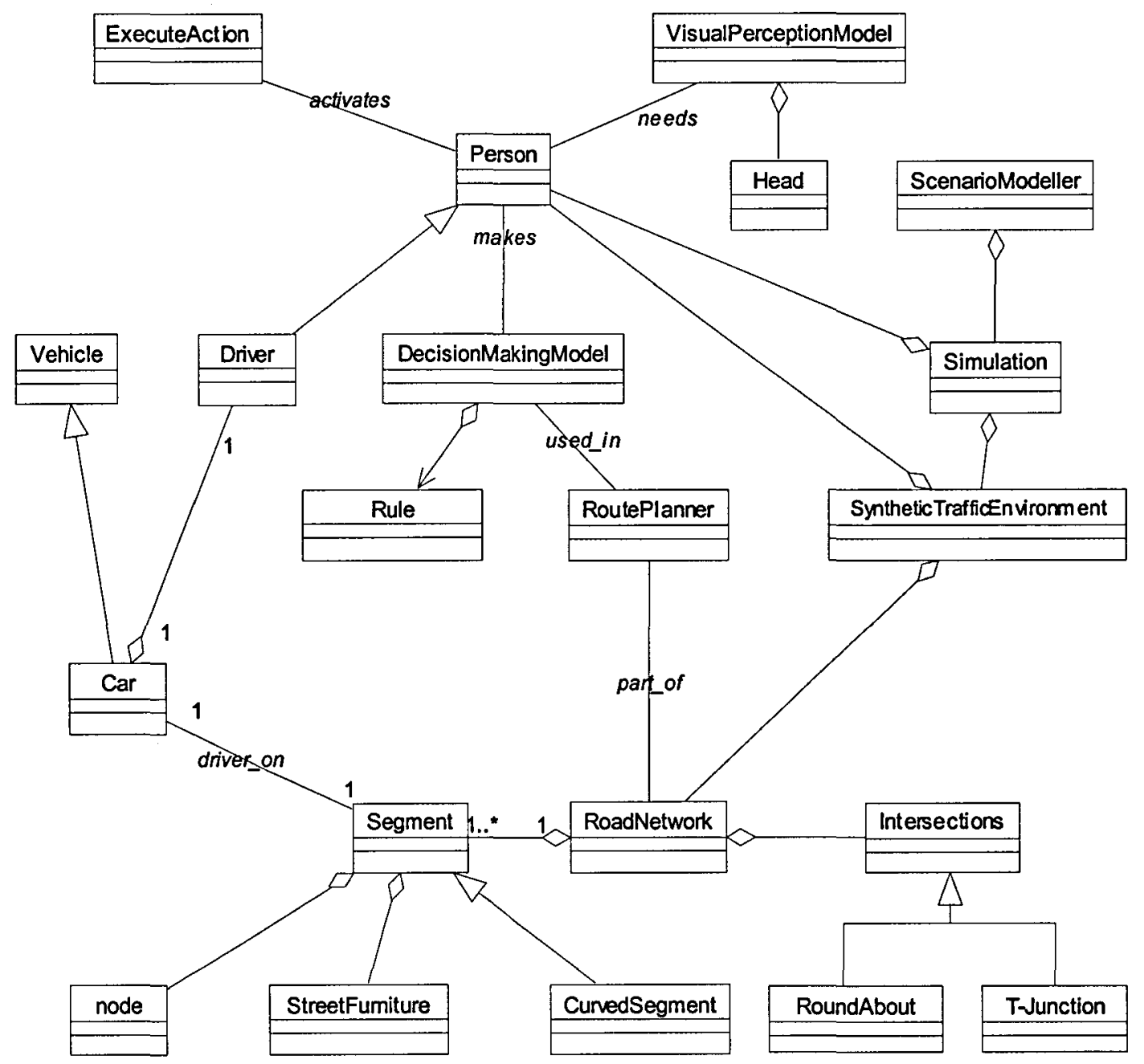

Figure 4.15: Class interactions within SD-SIM, using UML notation 
The Person class describes the categories of agents in the simulation in terms of driver, pedestrian, riders, etc. An instance of a Person is represented such that each person needs vision, makes decisions and takes actions. The VisualPerceptionModel class is responsible for acquiring visual information and then filters irrelevant information. This provides an input image to the driver and therefore has a one-to-one relationship between an instance of a driver and an instance of a Head. The VisualPerceptionModel is described by SEE as discussed in section 4.4. The DecisionMakingModel allows an instance of a Person to identify those Rules that are relevant and to assess the importance of each relevant rule. Those rules that emerge as relevant can be used_in the RoutPlanner class which forms part of the RoadNetwork. The RoutPlanner class is introduced here for completeness and for future development of Origin-Destination capabilities. It is currently not implemented as the author believes that route planning has a strong element of being prescriptive and interaction within the current version of SD-SIM should allow agents to take their own responsibilities. The ExecuteAction class models the basic control methods based on what decisions to execute.

Currently, the only type of Person that can be created is a Driver, although other types of people are defined as examples. This is because the focus in this thesis is on driver behaviour. However, because the basic attributes and behaviour are assigned to Person, it is easy to create other types of participating agents, inheriting the Person characteristics. Each Driver is associated with a Car. In other words, a car contains only a Driver at the moment. The Car class is derived from the base Vehicle class such that the Car class also inherits the attributes and functionalities of the Vehicle class. The Driver/Car unit is important in studying traffic interactions within SD-SIM, although examples of other vehicles such as buses, lorries, etc. are available.

The Car class is associated with a Segment class with the semantic relation drive_on. A Segment is defined by a start and end node and can also contain items of StreetFurniture e.g. sign posts, bollards, parked vehicles etc. A collection of logically connected Segments are used to define a RoadNetwork. The RoadNetwork class is made up of a collection of data that describe different road geometries e.g. Intersections such as Roundabout and T-Junction. The RoadNetwork and its relationship with the Segment are contained within the SyntheticTrafficEnvironment 
class. This class represents the general database class (e.g. the commercial graphics engine), which is responsible for the visualisation of the traffic environment. More details of this class are given in section 4.5.3.

Figure 4.16 illustrates the behaviour of the integrated framework, from the point of view of the Simulation class, using a state diagram. This diagram captures the different states of the Simulation object due to the various events that are processed by this class. Before the start of the simulation, initialisation is performed by specifying initialisation parameters within the ScenarioModeller. Based on the specified driving scenario (defined through the scenario modelling window), the simulation updates the synthetic traffic environment, drivers and cars. At the same time, updatePerson 0 calls three nested operations (VisualPerception, MakeDecision and ExecuteAction) to firstly acquire visual percepts from the environment, secondly to filter global visual information to eliminate irrelevant information (e.g. objects outside the driver agents' field of view) and support decision-making. Finally, based on the driving rules and the current visual information, appropriate actions are executed.

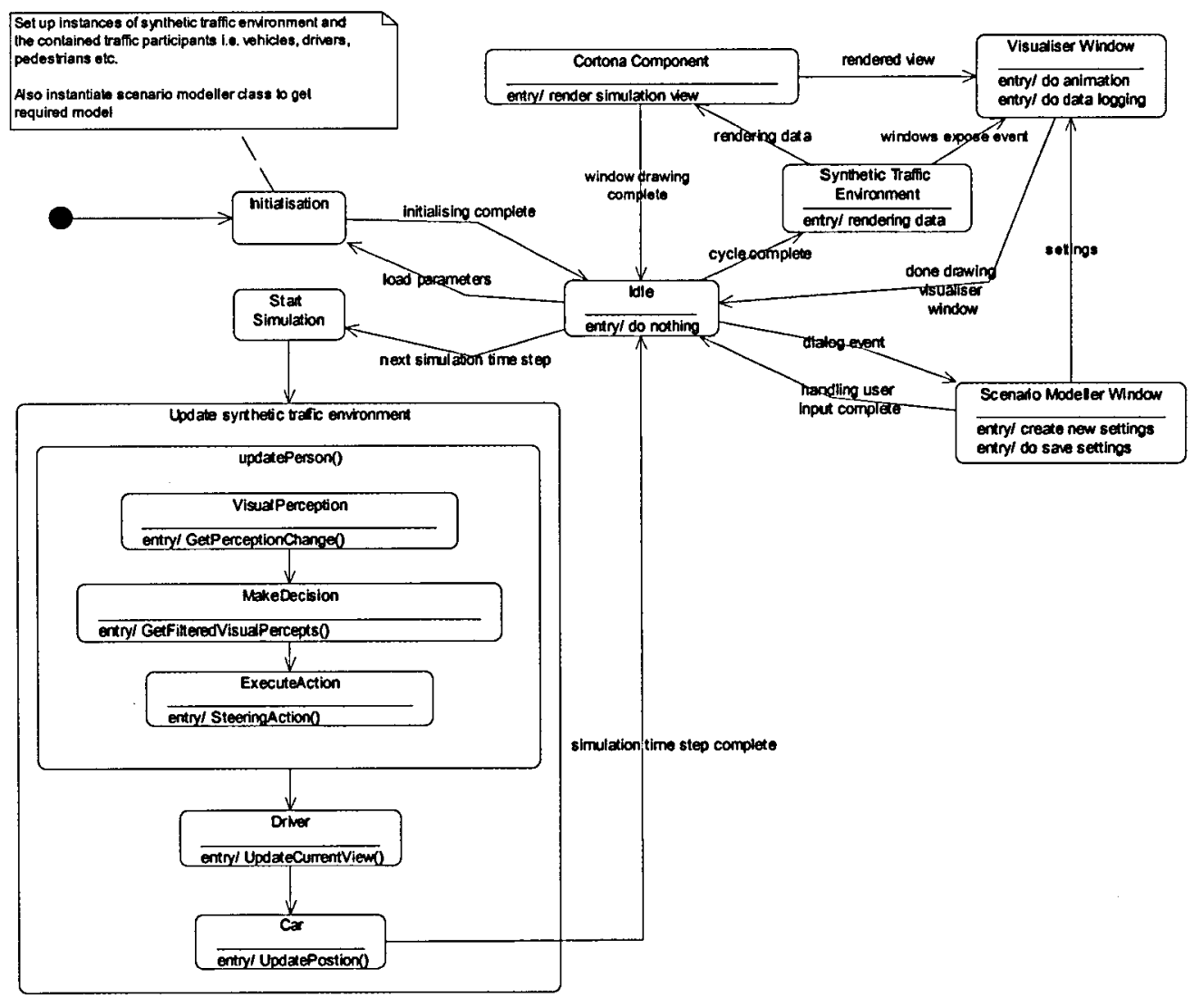

Figure 4.16: State diagram describing the simulation class behaviour 


\subsubsection{Scenario Modelling}

The ScenarioModeller class, which is part of the Simulation class, figure 4.15 , is responsible for configuring various parameters within the integrated simulation application. Scenario configuration in SD-SIM is based on a parameterised scenario authoring approach according to (Papelis 1996). This approach is consistent with the use of a Graphical User Interface (GUI). Therefore, by providing such an interface with the internal logic hidden away, there is flexibility in modelling different scenarios without the user needing to have programming skills. Furthermore, SD-SIM provides mechanisms for both interactive creation and management of scenarios and autonomous traffic. The sequence of interactions between the user and SD-SIM is performed in three main processes as demonstrated in figure 4.17. Each of these processes is explored further in the subsequent sections.

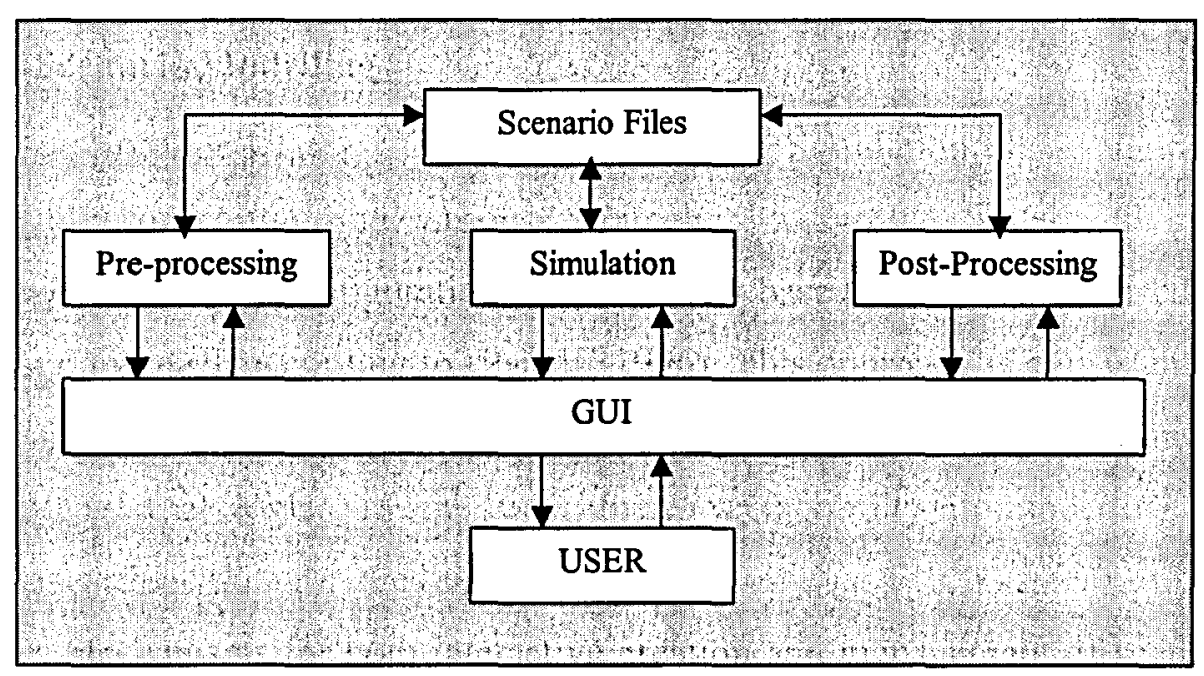

Figure 4.17: The main processes of modelling

\section{Creating Scenarios}

The first process, pre-processing involves the specification by the user, of initial and state parameters as part of scenario creation. Scenario for the purpose of this work is defined as a set of initial conditions along with calculated behaviour, in which simulation is the means of calculating the behaviour. Initial conditions refer to the initial conditions of each autonomous agent (e.g. driver, pedestrian and vehicle). A scenario can be created (for an entirely new scenario), opened (for a previously created scenario), saved (the scenario currently being worked on) and run. The procedure in creating scenarios is illustrated in figure 4.18. This is a 'Use Case' 
diagram from UML, which is an appropriate and useful tool for expressing scenarios from the point of view of the user's goals. In this kind of diagram, the actor or user of the system is shown as a 'stick figure' and the different scenarios are captured as use cases represented in oval shapes. The diagram also allows for relationships between the actor and the use case to be shown, including exceptions or alternatives.

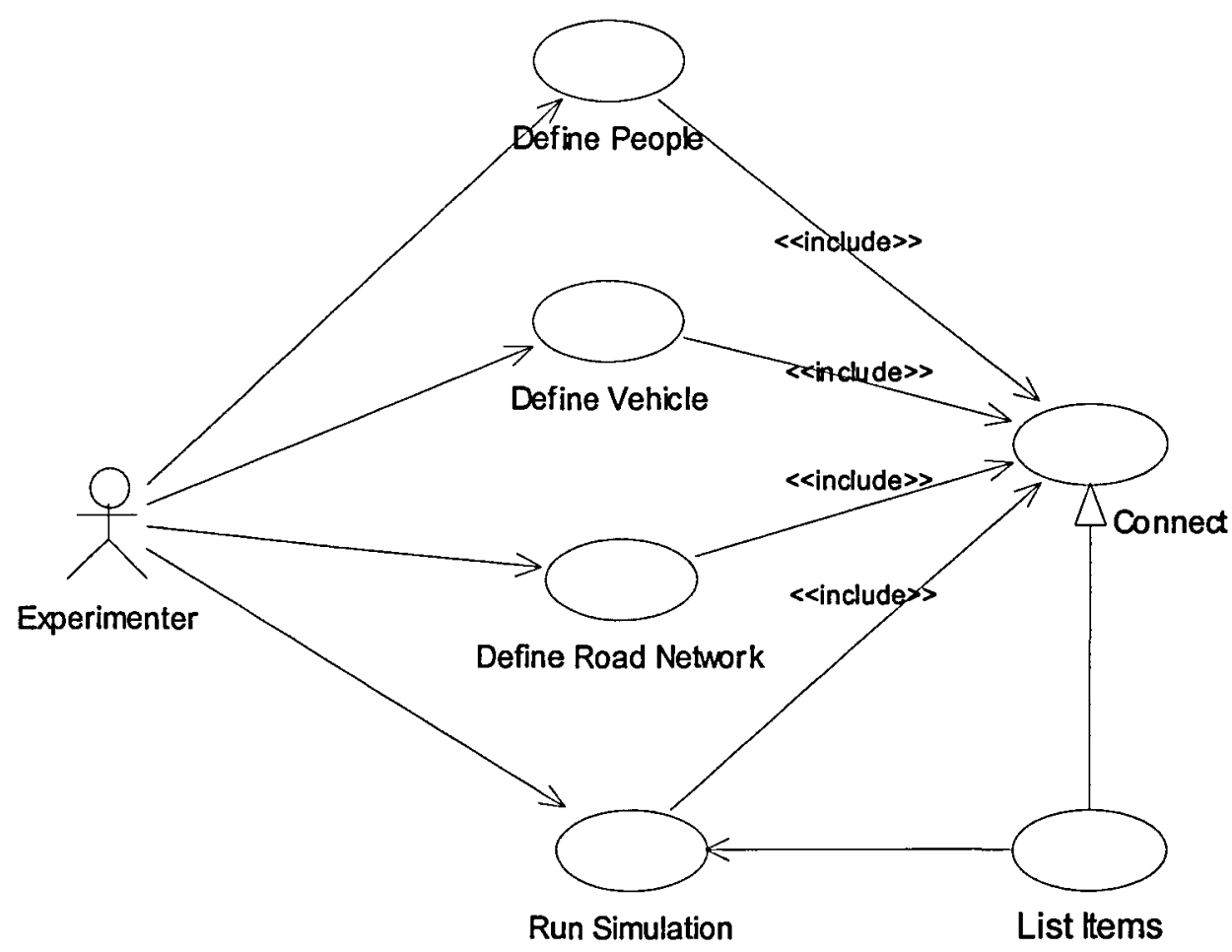

Figure 4.18: Defining scenarios through UML Use Cases

The main use cases in creating scenarios are Define People, Define Vehicle, Define Road Network and Run Simulation. In addition, different users e.g. traffic engineers, accident investigators, etc., of the system play a common role, for example to define a variety of scenarios and investigate the resulting interactions. Therefore, a generic name for a typical user is an experimenter. In Define People, the experimenter specifies parameters such as initial position, preferred speed, preferred forward and rear distances, preferred lane position, etc. for each driver agent. For Define Vehicle, initial geometry, kinematic and dynamics characteristics such as dimensions, maximum power, acceleration and brake fraction, are also specified. Road Network parameters include for example, road type, start and end coordinates, number of lanes, lane width etc. 
The next step is to specify the road and traffic events by linking Driver and Vehicle, Vehicle and Road Network, and Pedestrians and Road Network. This task is described within the Connect use case which is introduced to avoid describing the repeated process involved in linking the road/traffic events. Hence, its relationship with the other use cases is denoted by the semantic relationship $<<$ include $>>$. For example, to connect a vehicle to a driver, a unique identifier that associates the current driver to the corresponding vehicle is assigned. So if the current driver is number three, then a corresponding number three vehicle is selected. If either the driver or vehicle does not exist and an attempt is made to connect them, then a warning is given, prompting the user to create one. A similar process and check is carried out for connecting vehicles and the road network.

Before the simulation is run, the items that have been defined are collected within the List Item use case. This use case is similar to the Connect use case but provides additional functionality to add items such as vehicles, drivers and segments to the list, each time they are created and after they have been connected. As such, this is a generalised use case of Connect, inheriting some of the behaviour of the Connect use case. In this respect, Connect also represents an alternative scenario.

\section{Scenario Management}

Scenario management within SD-SIM is achieved through a number of facilities that allow the customisation and evaluation of scenarios. These include for example, copying output results into different applications like Excel, or switching drivers' viewpoints or displaying viewing data to determine what each traffic agent is seeing. An important aspect of SD-SIM's design is the way it handles and manages data within its different modules.

The internal data management of the scenarios is implemented in a hierarchical file structure containing vehicle properties, driver properties and road geometry descriptions as illustrated in figure 4.19. In this structure, the Project directory manages the way that data is handled within the system and contains one or more scenarios i.e. Scenario 1 to Scenario $n$ with each scenario having its own file extension. When a new Project is created, the GUI creates its own file path directory, saving scenarios within this directory. The Person, Vehicle and Network in each 
scenario are libraries containing a number of people, and vehicles. It is important to note however, that there is only one road network for the same equal number of people and vehicles since a road network can be made up of several segments. In addition, if people, vehicles or network in the current scenario are the same as those in a previous scenario, the network items must be copied from the previous to the current.

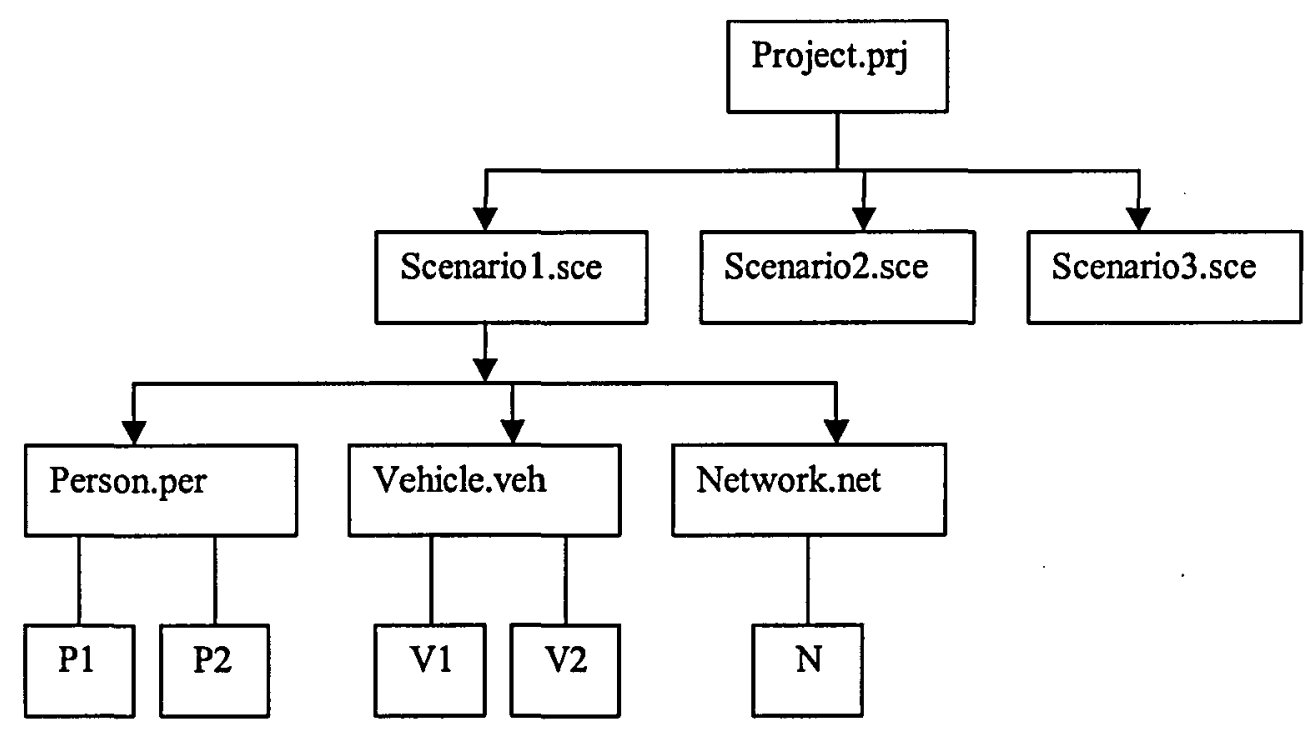

Figure 4.19: Hierarchical structure of a scenario data management.

The second part of scenario modelling in figure 4.17 is the simulation process, described in section 4.5.1, figure 4.16. After initialisation of parameters values as part of the scenario creation and management, the experimenter is then able to run the simulation. The List Item use case in figure 4.18 assembles the connected items to allow the simulation to run. The simulation process is also capable of flagging error warnings if the user failed to setup the data correctly and attempts to run the simulation. The functional design of the final process in figure 4.17 , i.e. postprocessing is now described in section 4.5.3.

\subsubsection{Animated Visualisation}

Post-processing is responsible for visualisation of simulation runs as well as displaying results to the user. As a compromise between reality and efficiency, the visualisation of STE is currently achieved by a Virtual Reality Modelling Language (VRML) based tool called ISA (Internet Scene Assembler) from (ParallelGraphics). 
This tool is loosely integrated within the common GUI through the definition of an interface between ISA and SD-SIM as illustrated in figure 4.20. This type of integration enables the two applications to coexist independently thereby providing the facility to plug in other more advanced visualisation tools in the future. The visualisation of the STE is a state in the simulation process figure 4.16 , which currently implements methods and properties of Windows Application Programming Interface - WinAPI to exchange information between a VRML scene and the GUI. A three dimensional scene is created in ISA including various buildings, trees, etc. and stored in a visual VRML database. Parameters for the simulated scenario are obtained from the simulation modules as part of the initialisation within the GUI. The methods and properties of WinAPI requests the VRML scene from the visual database and upon running the simulation, the calculated results can be visualised by launching the VRML file containing the scenario from the GUI. The WinAPI module uses this file and invokes the 3D Cortona (VRML browser) visualisation window.

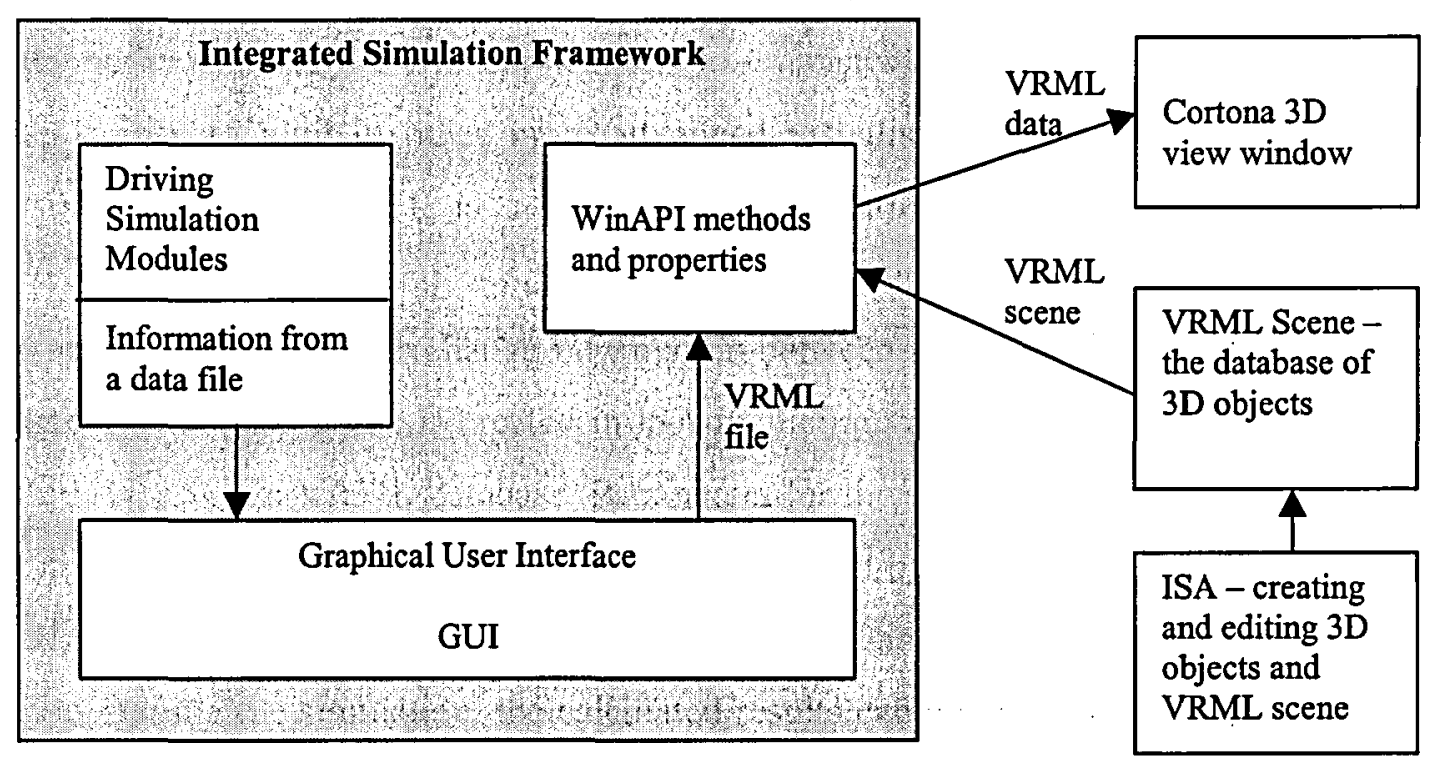

Figure 4.20: Visualisation components interactions

\subsection{Chapter Summary}

The functional design of a computational computer-based simulation tool called SDSIM has been described in this chapter. The design of this tool makes several contributions in fulfilling the modelling requirements and specifications set out in section 4.2. For example, the structure of SD-SIM and in particular, the IVD module 
is based on an agent abstraction, allowing virtual driver agents to have vision, decision and control functionalities. This enables individual agents to become autonomous and reactive to surrounding traffic. In addition, as a result of individual interactions, overall traffic flow emerges, thereby removing the need to explicitly prescribe the behaviour of the agents.

Visual perception for virtual agents is an important capability to support intelligent decision making within SD-SIM. As such, a detailed description of the functionality and mechanisms of the vision model has been given in this chapter. The overall design of the integrated simulation framework has been described using UML notation, consistent with traditional object oriented design methodologies. This provides an effective approach to foster software reusability and maintainability.

The ability to model different scenarios is important in the investigation of traffic flow interactions. Within SD-SIM, scenario configuration is achieved by a parameterised approach. The visualisation of calculated simulation parameters is achieved by simple VRML-based animations. Building on the design discussed here, chapter 5 describes the implementation of SD-SIM, demonstrating its capabilities. 


\section{CHAPTER 5}

\section{IMPLEMENTATION OF SYNTHETIC DRIVING SIMULATION}

\subsection{Introduction}

This chapter discusses implementation of the SD-SIM framework presented in chapter 4 , illustrated via a range of examples. SD-SIM's overall environment is demonstrated by its Graphical User Interface (GUI) which identifies the three major components discussed in section 4.3. As discussed in section 5.2, SD-SIM's user interface allows the user to define scenarios and provides a suite of facilities to support qualitative and quantitative visualisation of traffic flow interactions. Section 5.3 discusses the implementation of several key mechanisms for intelligent driver agent behaviour as well as demonstrating examples of SD-SIM's visualisation capabilities. The performance of SD-SIM in terms of its calibration and assessment, including real life scenario modelling is discussed in chapter 6 .

\subsection{The Graphical User Interface}

The graphical environment of SD-SIM is illustrated in figure 5.1. User interaction with SD-SIM is achieved mainly through this GUI by specifying parameters as discussed in section 4.5.2. There are six pull-down menu options available to the user: (1) File (2) Edit (3) Options (4) Visualisation (5) Windows and (6) Help. Each of these contains several items, some of which are not fully implemented. For example import and export facilities in the visualisation menu. This is because of the author's belief that the focus in the implementation of SD-SIM must be to provide key functionality that fulfils the requirements set out in chapter 1 and section 4.2. In addition, because an object oriented design and implementation has been adopted, it is possible to extend SD-SIM's features and functionality by simply adding more objects or classes in the future. 


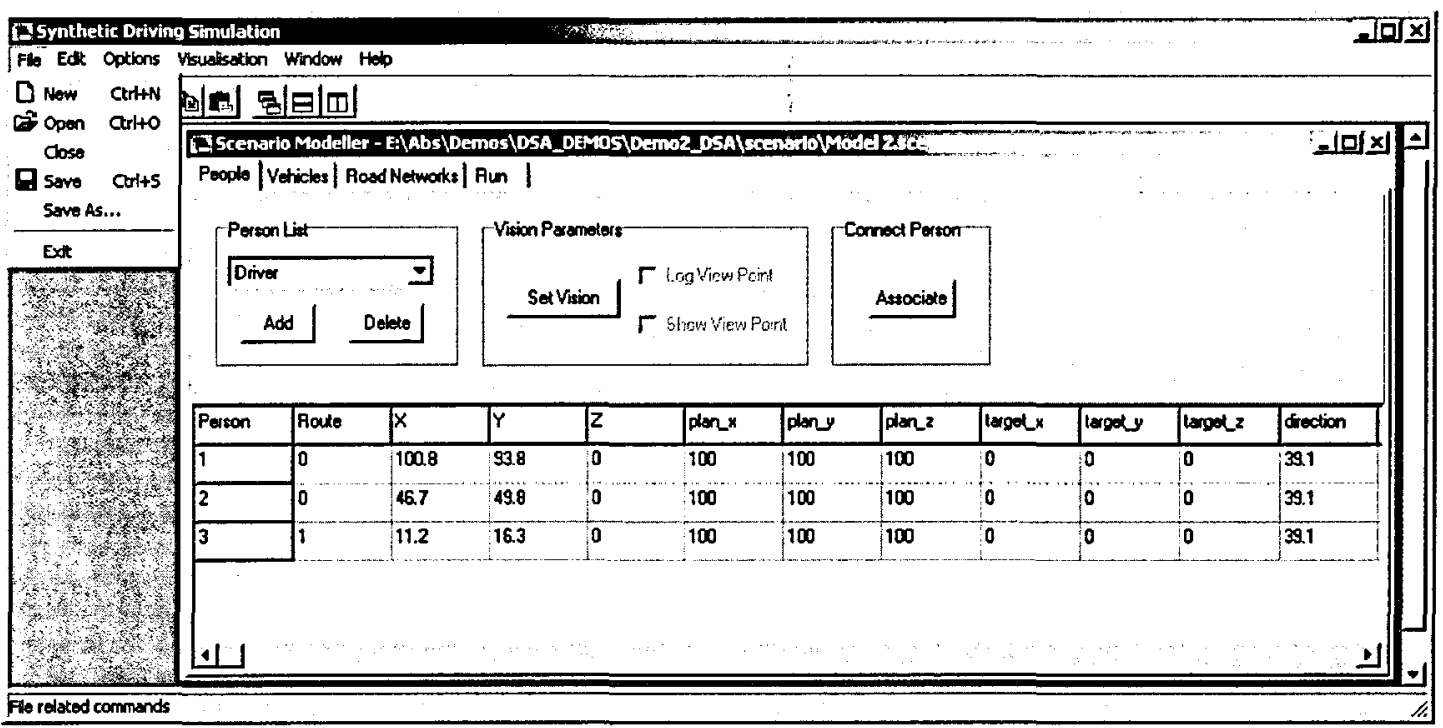

Figure 5.1: Main graphical user interface of SD-SIM

Currently, the File option is the main menu of the simulation GUI and contains items New, Open, Close, Save, Save As and Exit. The New item loads the Scenario Modeller window as shown in figure 5.1. This is a child window and contains a default simulation scenario which the user can run, modify and save. The title of this window is intended to show the current model e.g. Scenario Modeller - Model 2 where Model 2 is the name of the current model. More details of the Scenario modeller are given in sections 5.2.1, 5.2.2 and 5.2.3. The Open item in the File menu opens a previous simulation model if one exists. The Save option allows changes to the current model to be saved thereby overwriting the previous parameters and settings. When the Save As option is selected, a standard Save As window (shown in figure 5.2) appears so that the user can save the new model. Within this window, there is a directory to put the files in. This directory contains a library of files as explained in the Scenario Modelling section of 4.5.2. However, since this a standard window, the user can change directory with the drop-menu to select any new directory of their choice. The types of files supported in this window are SD-SIM's own scenario generation extension, e.g. .sce. When a model is saved, the software creates a new scenario extension file and a model directory corresponding to that scenario. 


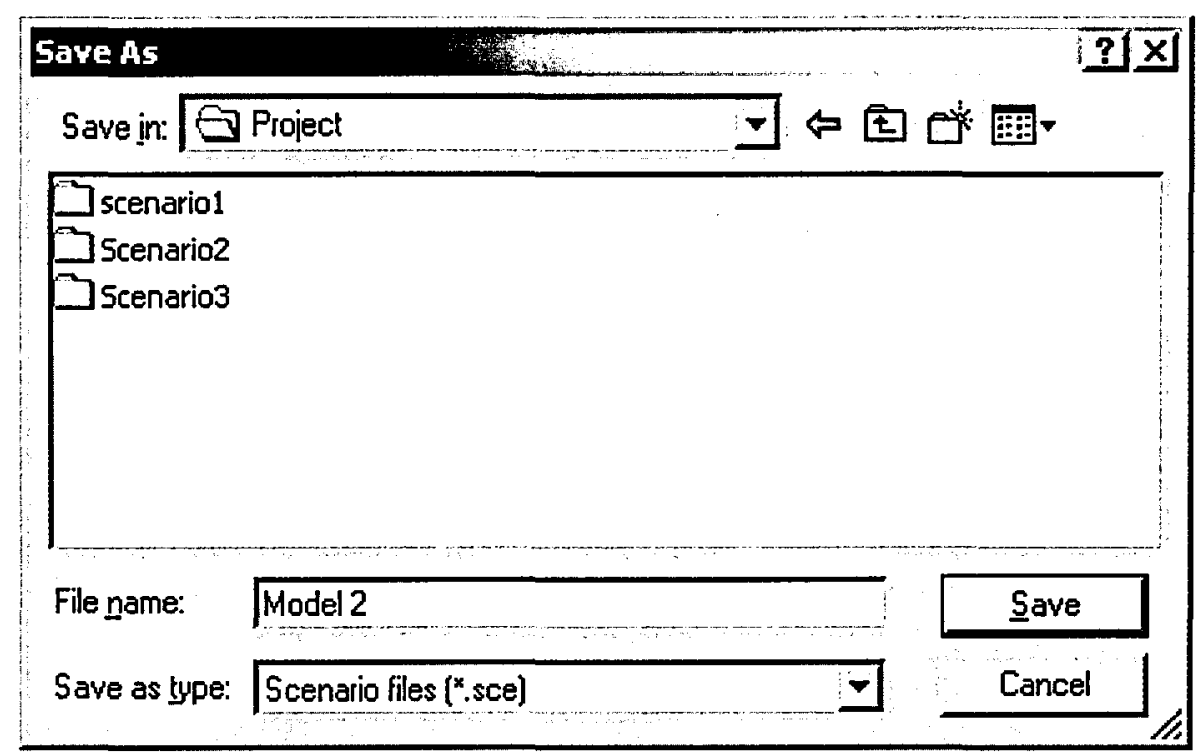

Figure 5.2: Implemented hierarchical saving functionality

\subsubsection{Configuration of People}

In the process of creating a new scenario, the Scenario Modeller window contains four tabs, figure 5.1. The tab implementation provides flexibility to the user to define People, Vehicles and Road Networks in any order. The first tab provides the facility to configure the people in the simulation. In the People window, a Person List box shows the category of people that can be defined in the simulation e.g. Drivers, Pedestrians and Motorcyclists. Since, items in different tabs can be defined in any order, there needs to be a facility to connect these items, hence the Associate button enables a driver to be connected to a vehicle through the Connect Driver window. In this window the user connects the different items by specifying an identification number (e.g. driver "1" is associated to car " 1 ") and confirms this by pressing the OK button or cancels by pressing the Cancel button. However, if cancel is selected and no connection exists at run time, an error message is flagged to warn the user to first perform the connection. Vision parameters can be defined for each category of person through the Set Vision button. The button opens a Vision Parameter window (figure 5.3) in which various parameters for Angular Head Movements, Eye Dimensions, Eye Resolution and Field of View for a particular selected driver to be defined. 
The People tab is the default window when the Scenario Modeller is activated and this tab also corresponds to a table in the Scenario Modeller window, figure 5.1. This table has the number of people (Person) as rows and the different parameters that characterise the individual person as columns. People can be added to or deleted from the table with the relevant buttons. The Add button creates a new person with parameters on a row which the user can edit. Although a Person actually refers to a driver in this current version of SD-SIM, it is the author's opinion that a generic name which refers to Person instead of Driver is in fact more appropriate. This is because, a Driver is a type of Person but also when a Pedestrian is selected from the Person List, the table corresponding to a Pedestrian Person should be loaded. However this is not yet implemented as the author believes the current focus of the work must be on the IVD (section 4.3.1). An example of some of the parameters that can be defined for a driver person in this table are $\mathrm{X}, \mathrm{Y}, \mathrm{Z}$ position, driver's plan i.e. his/her target position, current and target speeds, rules and weights, etc.

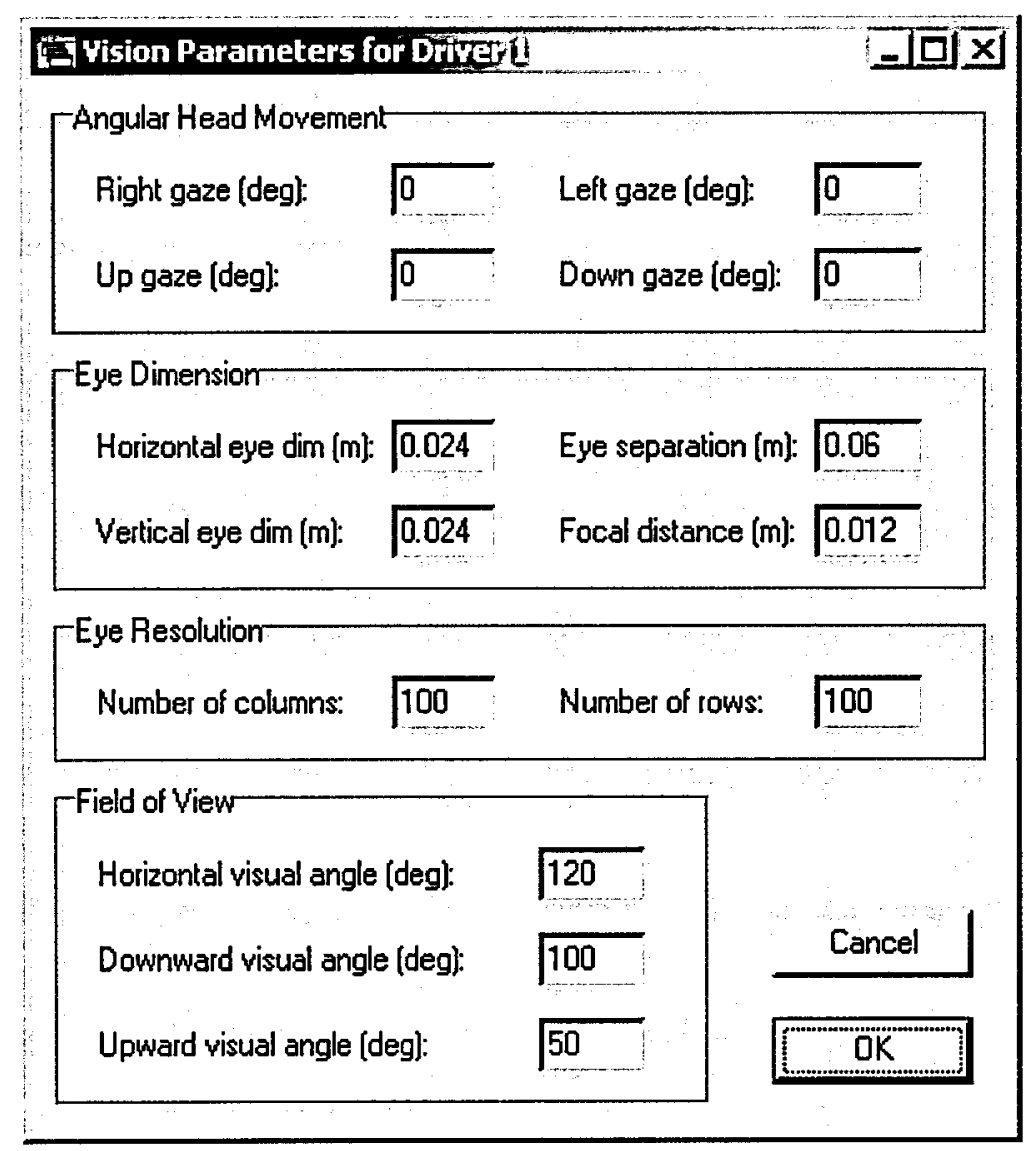

Figure 5.3: Vision parameter window 


\subsubsection{Vehicle Configuration}

In the process of creating a new scenario, the user must also create and configure vehicle parameters. This follows a similar process to the configuration of people using the Scenario Modeller. The process starts with the user clicking the Vehicles tab which loads a similar table window as the Person window, as shown in figure 5.4. In this window, a type of vehicle is selected from the Vehicle List. Once again, although examples of vehicles such as Bus, Heavy Goods Vehicle, Bicycle, Motorcycle, are defined in this list, only Car is currently available. A number of vehicles can be created through the Add button or deleted using the Delete button. The different initialised parameters of a Car Vehicle type are listed. Some of these include, the X, $\mathrm{Y}, \mathrm{Z}$ initial position of the vehicle, which corresponds to the Driver position since a driver is located in a vehicle.

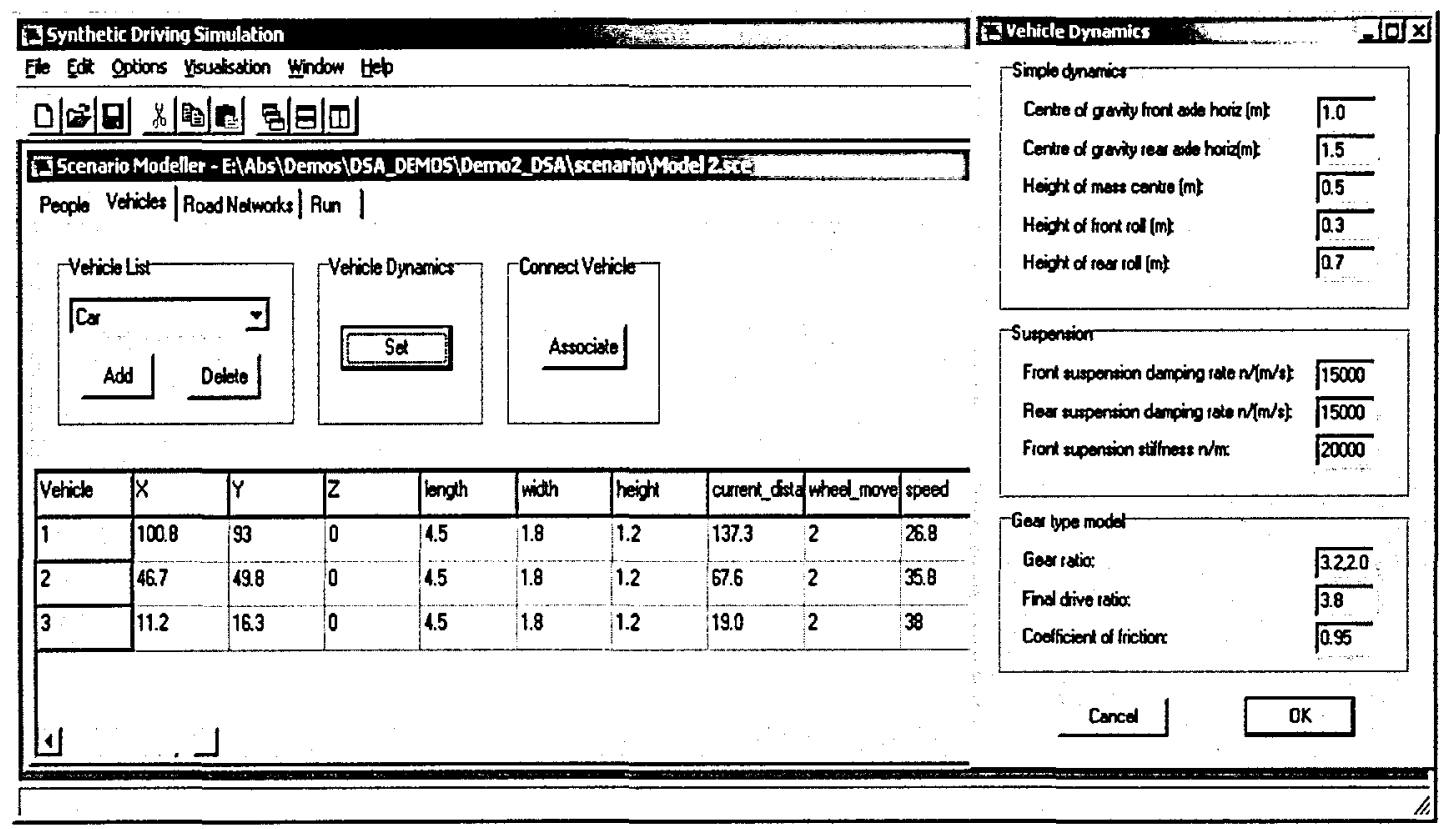

Figure 5.4: Vehicle configuration

SD-SIM currently implements simple vehicle characteristics such as braking, acceleration, gear change, steering and maximum power. The characteristics for advanced vehicle dynamics within SD-SIM have been added to take into account the functional requirements of the new On-The-Spot (OTS) research project discussed in section 4.3.2. 
The defined or configured vehicle must be associated with a driver and connected on the road network. This is achieved by the Associate button of the Connect Vehicle section of the Vehicle tab. A Connect Vehicle window is opened in which the user specifies identification for the vehicle item to be connected. As a check, if either a driver or a network had not been previously created, a warning message is flagged when the user attempts to confirm the connection through the OK button. This allows the user to go back and create the necessary associations for driver and network. The third property to be configured within SD-SIM is the components of the synthetic environment part of which is represented by the road network.

\subsubsection{Road Network Configuration}

The road network configuration involves the user clicking on the Road Networks tab to define properties for a single segment component. This loads a segment table window from which a network component can be selected from the list. Currently, only a straight segment can be defined. This segment is made up of a number of lanes, lane width, start and end coordinates and total length of segments. The number of lanes and lane width are representative of a typical British motorway.

People and Vehicles are connected onto the network from the Associate People and Associate Vehicles buttons respectively. This is achieved by specifying corresponding identification numbers for both vehicles and people. After setting up the geometrical properties of the road network and logical connection information within SD-SIM, an integrated VRML tool described in section 4.5.3, is used to enrich the synthetic traffic environment by adding environmental objects such as trees, buildings, background or skyline colour, road texture, etc.

\subsubsection{Qualitative Versus Quantitative Visualisation}

SD-SIM aims to reflect reality as closely as possible. Consequently, visualisation is essential in evaluating, analysing and understanding the realism of simulated driver interactions and traffic flow within SD-SIM. In this respect, understanding is enhanced through interactive techniques as well as qualitative and quantitative visualisation. SD-SIM's user interface is implemented to support interactive creation 
and manipulation of scenarios as well as provide a suite of facilities for qualitative and quantitative visualisation. These techniques allow various driver interactions and the algorithms that support driver behaviour to be investigated. Some of the interactive configuration facilities of SD-SIM have been discussed in sections 4.5.2 and 5.2. Figure 5.5 illustrates qualitative and quantitative visualisation of calculated behavioural data produced in SD-SIM. A brief discussion of the qualitative and quantitative visualisation criteria that underpins SD-SIM's implementation now follows, with the end of this section providing detailed description of figure 5.5.

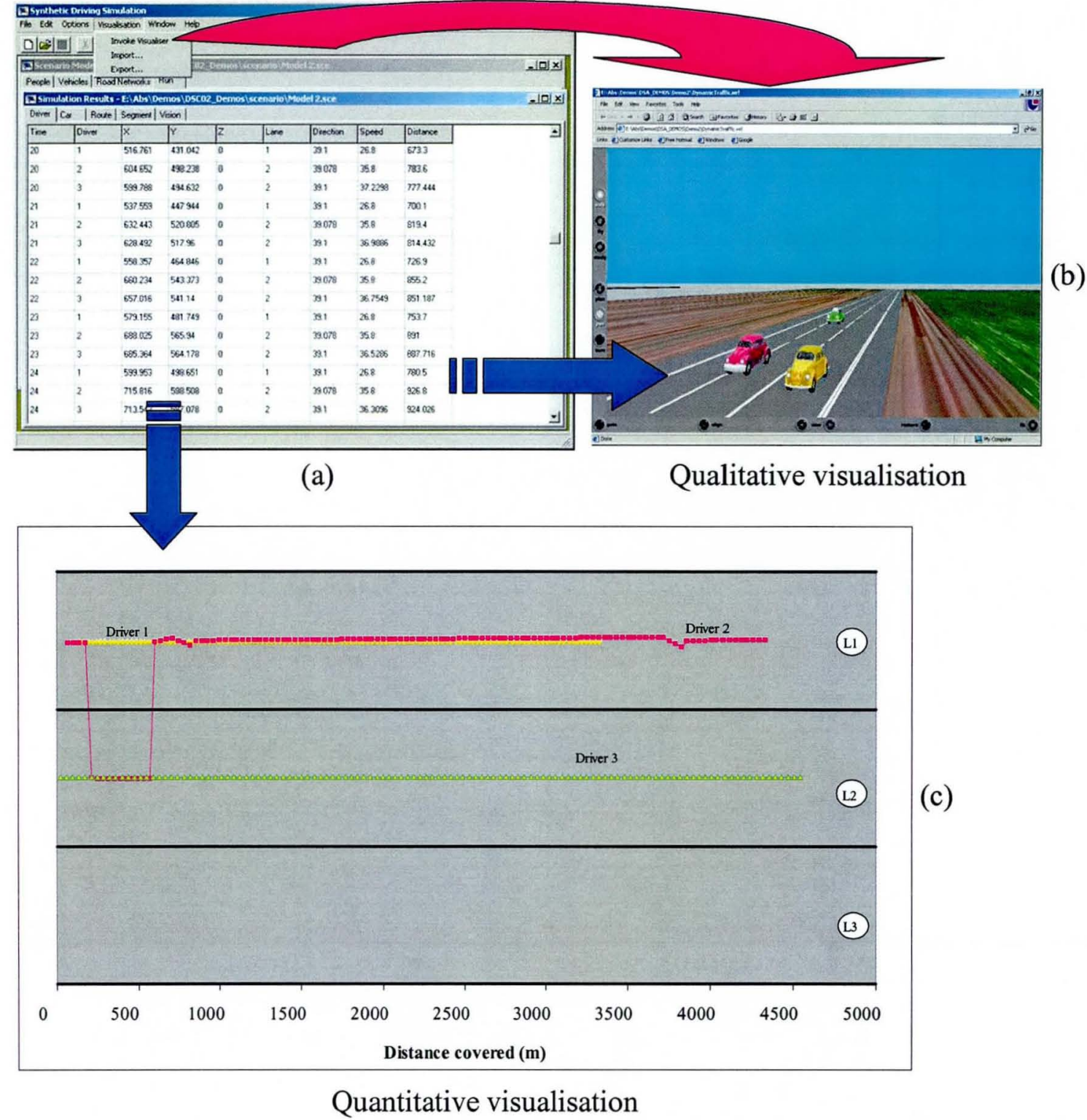

Figure 5.5 Qualitative and quantitative visualisation within SD-SIM 
Qualitative visualisation describes situations in a non numerical way, by comparing simulated behaviour with reality and expectation. This is particularly important in evaluating complex dynamic systems such as driving. The visualisation development environment (e.g. VRML animation) is often a good technique used to convey qualitative information. In SD-SIM, qualitative visualisation is demonstrated through graphical presentation and animation techniques to verify whether the behaviour of the virtual driver agents is reasonable and in accordance with the designed algorithms. Visualisation is achieved by the user invoking the Visualiser from SD-SIM's pulldown menu. This loads an animation of the current simulation into the integrated web-based VRML visualisation tool. The user can choose to investigate what individual drivers are 'seeing' in each time step by using a pull down menu and then use a slider to select the different driver's view from the user interface. Alternatively, different and multiple camera views including each driver's view or a bird's eye view can be created and each view examined (compare figures 5.5 and 5.12).

However, the realistic impression of qualitative visualisation is influenced by a number of factors including the graphics engine and visualisation database. For instance, high fidelity visualisation tends to require high end commercial tools such as MultiGen, or the Open Inventor graphics library. These tools are, however, expensive and are also limited in the way 3D graphical information has to be manipulated. Factors that influenced the implementation of SD-SIM's visualisation are based on the following:

- Availability and cost of the visualisation tool. The availability of the tool should be balanced with the cost of the tool. Also, the tool should support basic features such as overview windows, synchronisation and multiple views.

- Compromise between reality and efficiency. As well as cheap and efficient visualisation capability, the tool should be able to support realistic animated visualisation of behavioural data calculated by SD-SIM.

- Interactive nature of the tool. The visualisation tool should provide facilities (e.g. menus, buttons, etc.) to manipulate various views or provide navigation within the visualisation environment to support the understanding of qualitative evaluations.

- Data handling and output of the visualisation. The tool should be able to handle data exchange in an integrated manner. For example, file exchange between 
different file formats, in this case, loading SD-SIM's data files into the Virtual Reality Modelling Language (VRML) file format.

In addition to essential qualitative visualisation, quantitative visualisation enables the extraction and analyses of parameterised values to explain complex interactions between the driver agents. Quantitative visualisation tends to use statistical techniques or analysis of charts and graphs in spreadsheet applications to calculate the numerical difference between reality and simulation. In general, simulation within SD-SIM utilises verifiable calculations. The main Simulation Result window shows the calculated behavioural data generated by SD-SIM. Figure 5.5(a) illustrates typical quantitative visualisation of SD-SIM behavioural data; a tabular display of calculated data showing simulation time steps, driver type, $\mathrm{X}, \mathrm{Y}, \mathrm{Z}$ positions of the driver/vehicle unit, lane position, direction, speed and distances covered (absolute). Figure $5.5 \mathrm{~b}$ depicts qualitative visualisation of the same results (in the form of simple virtual reality animations). SD-SIM supports quantitative visualisation by allowing the user to copy and past simulation data into an Excel spreadsheet. This data can then be analysed using Excel's graph facilities to reveal the circumstances that have led to the drivers' interactions. For example figure 5.5(c) shows an alternative quantitative visualisation of the same results in figure 5.5(a), as Excel plots of each driver's distance and lane position profile. Also, the calculated vision parameters can be displaced in a text field or written to a log file when the user specifies this on the check boxes. Visualisation of vision parameters is illustrated in figure 5.12.

\subsection{Modelling and Visualisation of Intelligent Driver Behaviour}

This section discusses implementation of key mechanisms of intelligent virtual driver behaviour in SD-SIM. Such intelligent behaviour is defined here according to (Albus 1991), who suggest that at a minimum level, intelligence requires the ability to sense the environment, to make decisions and to control action. These elements provide a suitable context in which to demonstrate through qualitative and quantitative visualisation the key features of intelligent behaviour. 


\subsubsection{Binary Image Capture}

As described in section 4.3, each driver agent is capable of perceiving its environment through the VPM. The VPM includes SEE which is responsible for both explicit object detection and visual information processing. Object detection is of critical importance for subsequent information processing, decisions making and execution of actions. Object detection is implemented in the CalculateView() algorithm. The method CalculateView() is passed head and object information when it calls the See() method. The See() method is responsible for performing ray tracing calculations to determine the visibility of objects within the driver agent's field of view. As such, this method introduces uncertainty through limitations (e.g. objects outside the range and field of view are not detected) imposed by the agent's range and field of view. A binary 2D image map of all detected objects is constructed by the SetFacetPixels() method. The rendered image is then mapped into a 16-plane 'visual memory' in successive time steps for further information processing.

In the implementation of SEE, several sets of test data have been devised to demonstrate object detection. Figure 5.6 show typical example of head and object (vehicle) input data used for the test runs. In these test runs, the observing driver is driver 2 and the head input data for driver 2 specifies a 50 × 5 matrix for each eye (figure 5.6a). Driver 2 was initially $50 \mathrm{~m}$ (figure 5.6b) away from the target vehicle. The target vehicle has dimensions of length $3 \mathrm{~m}$, width $1.8 \mathrm{~m}$ and height $1.5 \mathrm{~m}$. The observing driver is approaching the moving target driving.

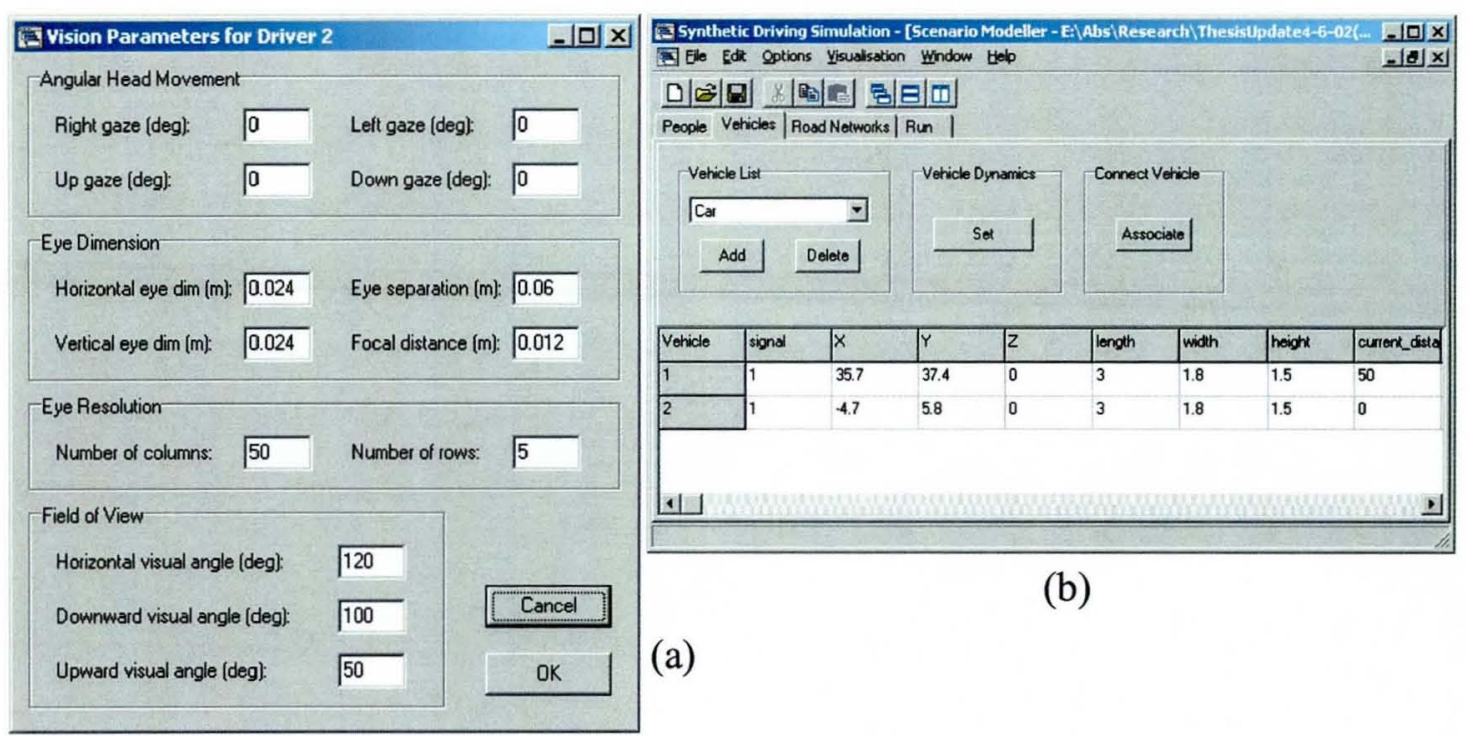

Figure 5.6 Object and head definition files 
The simulation was run for 60 time steps and the resulting outputs to these inputs for the first time step is given in figure 5.7. The detection of the image of the target vehicle is depicted in minimal form by a simple square or rectangle added in binary 1. Since the target vehicle was initially at a long distance away, only a few rays are hitting it. Each eye registers an image of the object and the ProcessImage() method combines the images from each eye to determine the total number of objects detected.

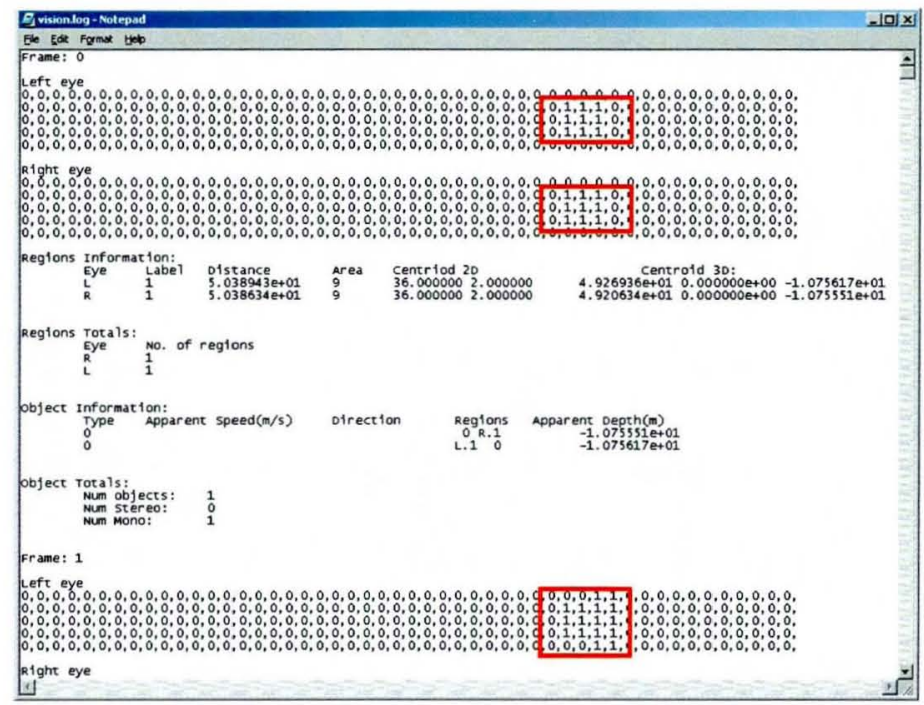

Figure 5.7 Objects detection and counting

Keeping the head definition of figure 5.6 the same, more objects are added to the object definition file as shown in figure 5.8 to further demonstrate object detection and counting. A maximum number of five objects were presented to driver 2 .

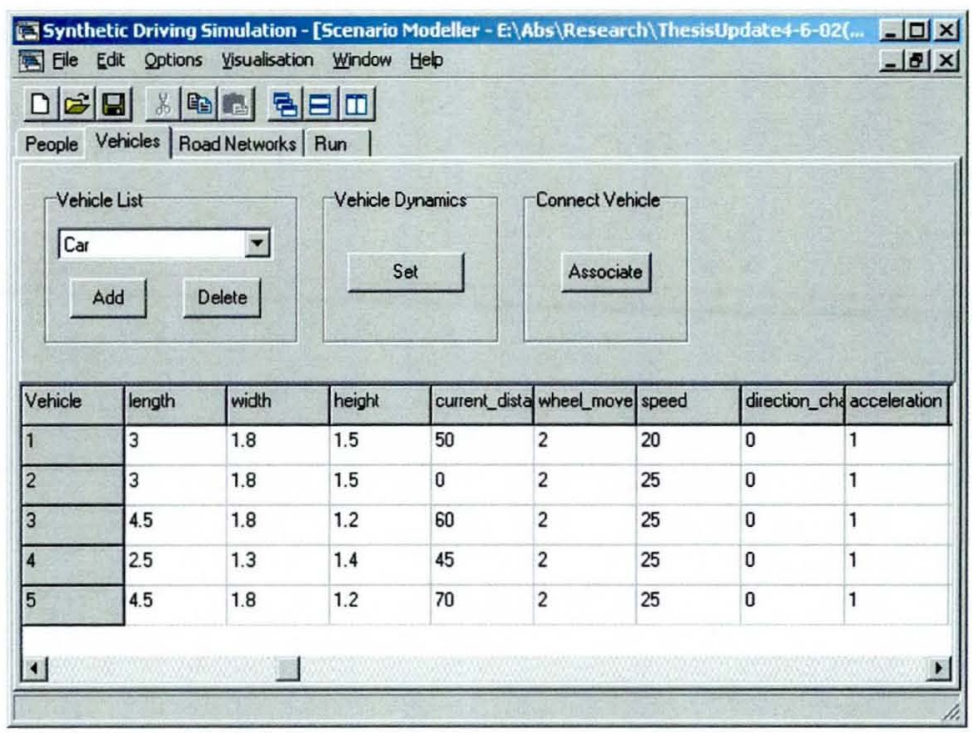

Figure 5.8 Object definition files 
Figure 5.9 shows the performance of driver 2 using SEE to count the number of objects presented to it at each run. From the graph, desired output refers to the number of defined objects and actual output refers to the vision model's output. Figure 5.9 indicates very important aspects of the vision model. The test runs are carried out using different object input data to highlight the limitations of the SEE. So in the $3^{\text {rd }}$ test run, the graph shows that although 3 objects were presented to the eyes, SEE actually detects 5 objects. This is because the current counting algorithm relies on a 4connectivity scheme (see section 4.4.3.1) and does not work as well as an 8connectivity labelling scheme especially when additional object features or 'noise' is present. This often leads to a miscount. Although the graph demonstrates object counting, in driving simulation, virtual driver agents rely less on object counting than other forms of visual perception. Object detection and classification provide more useful information about the traffic environment to support decision making.

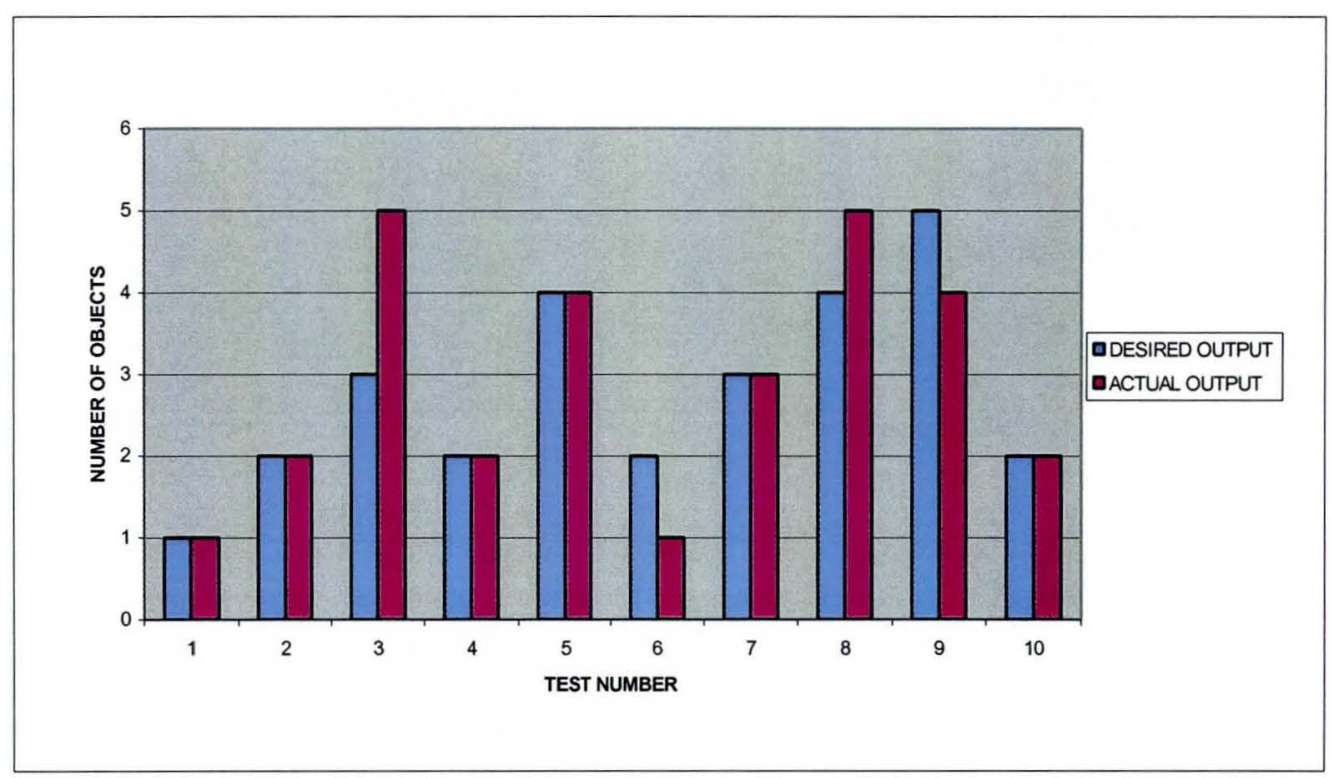

Figure 5.9 Determine numbers of objects detected in both eyes

\subsubsection{Visual Information Processing}

Visual information processing is distinguished from the object detection process. The implementation of visual information processing ensures that individual driver agents maintain a distinct representation of their environment through the execution of several algorithms. The key tasks of the visual information processing performed by each driver agent are; image identification, stereo/mono processing, image tracking, distance and speed perception and motion perception. Image identification is 
implemented in the SegmentProcess() method and ensures that connected image regions are identified and labelled. Within the image identification process, some images maybe occluded by others which, as a consequence may affect lower level processes. Therefore an OcclusionDetection() method is implemented to detect and remove image occlusion.

Since each driver agent has two eyes, it is necessary that they are capable of processing stereo images that have been captured and segmented in the eyes. Therefore, a CreateObjectCue() method is implemented to perform stereo analysis. The processed stereo images provide information for apparent depth due to the effect of both eyes seeing the same object. Sometimes only one eye can see an object. This is usually the case where an object happens to be in the peripheral view or in other words outside the defined stereo region. When this happens, the stereo algorithm is automatically modified to produce mono information such as actual distance to the detected object instead of its apparent depth.

Image tracking is implemented using the ObjectTracking() method which is responsible for virtual driver agents maintaining a distinct observation of the objects in their environment, taking into account the dynamic nature of the situation. Virtual driver agents are able to track images from frame to frame to extract useful information e.g. object displacement that is passed to the distance, speed and motion perception tasks. These tasks are based on subjective interpretation of visual information, for example, distance perception or depth is determined by analysing consecutive image patterns due to stereo analysis. In addition, apparent speed is inferred by calculating the displacement of each tracked image in the eyes over a given time step.

The capability of SegmentProcess(), CreateObjectCue() and ObjectTracking() implementations for the classification objects is illustrated in figure 5.10. SD-SIM was set up with five vehicles (objects), moving within the field of view of an observing stationary driver. The intention of this scenario was to test the classifying algorithm. The observing driver was given an eye resolution of 100 columns x 50 rows. The driver agents utilise cognitive models - internal representation of the knowledge of vehicle dimensions to determine the type of vehicle detected in each 
time step. The classification scheme currently employs the aspect ratio of objects by comparing similar properties such as length, width and height against internal representation of vehicle properties.

The graph in figure 5.10 shows the accuracy with which objects detected in both eyes are classified into three categories of Cars, Lorries and Unknown. The graph shows that $66 \%$ and $33 \%$ of objects are classified correctly as Cars and Lorries respectively. Only $1 \%$ of the objects presented to the eyes cannot be classified correctly. Also, when "noisy" inputs (those inputs with rotated objects) are used, the vision model has a $60 \%$ reliability of classifying these objects. The classification reliability is much higher for inputs with no "noise".

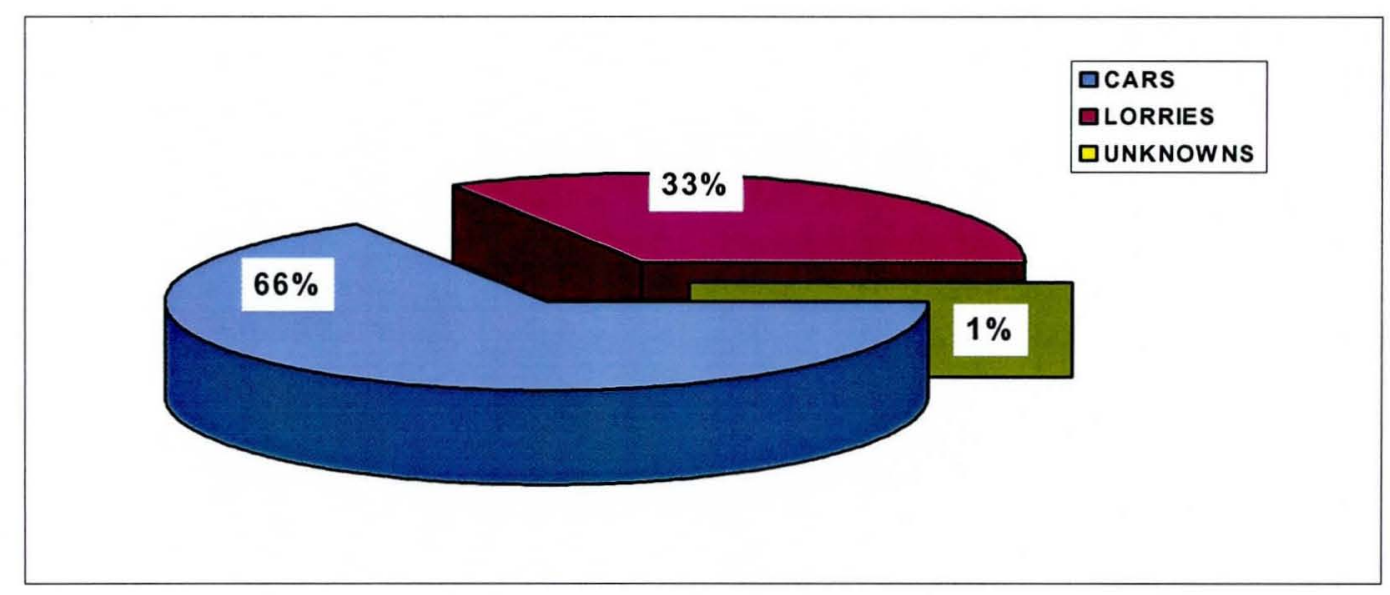

Figure 5.10 Classifying objects

\subsubsection{Influence of Vision on Modelled Driver Decisions and Traffic Flow}

The current information perceived from the environment is passed to the decision making module. The implementation of driver decision making is based on a number of internal parameters (e.g. rule use values, random numbers) and external parameters (e.g. rule weights), section 4.3.1.2. As a result, the current implementation suffers from an inherent problem in evaluating the combination of rules that has led to the current outcome. Also, physically selecting appropriate parameters to produce desired outcome is often a time consuming process. 


\subsubsection{Example Scenarios}

The aim in this section is to demonstrate implementation examples that show the influence of vision on driver decisions, in turn leading to improved simulation of driver interaction and traffic flow. Two scenarios have been designed to exemplify distance and speed perception, motion and looming detection, car following and lane changing.

\section{Scenario 1}

The first scenario involved two virtual driver agents. As part of the initial scenario configuration, driver 2 was initially behind driver 1 and assigned a preferred front distance of $40.0 \mathrm{~m}$, preferred speed of $35.8 \mathrm{~m} / \mathrm{s}$. Driver 1 was assigned a preferred speed of $26.8 \mathrm{~m} / \mathrm{s}$. Both drivers initially occupy the same lane i.e. lane 1 . Driver 1 had a preferred lane of lane 1 whilst driver 2 preference to change lane was weighted higher i.e. 1.0. The simulation was run for unsighted (without vision model capability) and sighted (vision model) driver 2. Each driver in scenario 1 was given a horizontal versus vertical eye resolution of 50 by 5 . The choice of eye resolution was based on the need to balance out computational efficiency and adequate accuracy. The first ten time steps provided opportunity to analysis sighted and unsighted driver 2 .

\section{Scenario 2}

The second example scenario was set up for three drivers as shown in figure 5.11. Drivers 1 and 3 were placed in lanes L2 and L3, respectively, both 50m in front of driver 2 (lane L1). The preferred front distance for each driver is $20 \mathrm{~m}$ and their speeds are: driver $1-20.0 \mathrm{~m} / \mathrm{s}$, drivers 2 and $3-25.0 \mathrm{~m} / \mathrm{s}$. Furthermore, each driver was given a horizontal versus vertical eye resolution of 100 by 10 . Drivers 1 and 3 have similar rule weightings that are different from driver 2. For example, the need to maintain minimum headway is weighted higher at 0.9 for drivers 1 and 3 and 0.8 for driver 2 . 


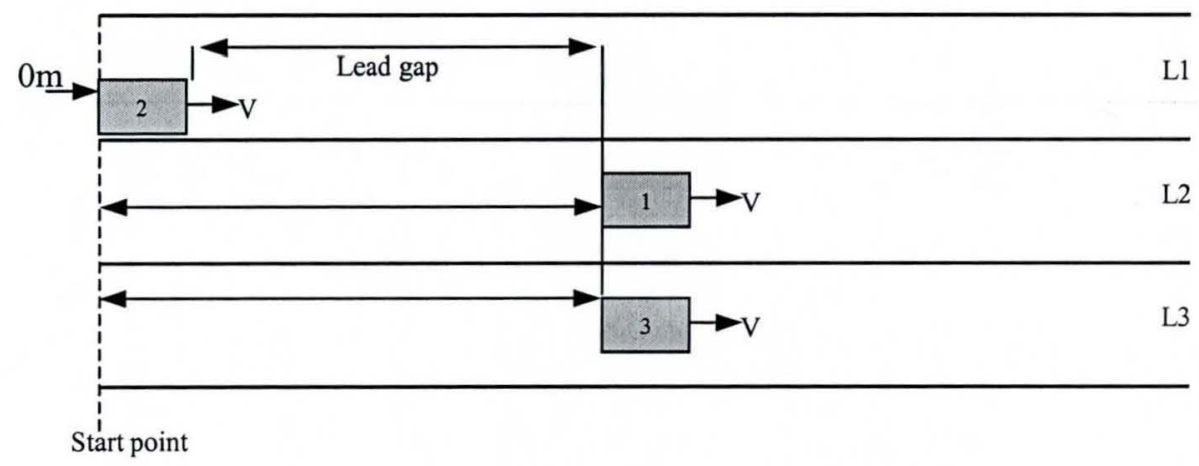

Figure 5.11 Three driver interaction scenario

Figure 5.12 illustrates the use of qualitative and quantitative visualisation of driver vision and behaviour in scenario 1. For example driver 2 (magenta car) in figure 5.12, detects (shown as two blue bitmap images in the left and right eyes of the vision window, left picture) the presence of driver 1 (yellow car), using the detection algorithm described in chapter 4. Employing speed and distance perception heuristics described in chapter 4, driver 2 infers (estimates) the speed of driver 1 to be slower than his speed and the distance between them to be decreasing. Since driver 2 is approaching faster, at some later point in time he also experiences a looming effect (expansion of image area between figures (a) and (b)) such that the image area registered on the 'retina' of the eyes correlates to perceived distance. Driver 2 uses this information along with preferred parameters (e.g. to maintain minimum headway etc.) to investigate any impending collision (using collision detection and avoidance algorithms in section 4.4.3.2) with driver 1 or the infringement of driver 2's preferred front distance by driver 1 . If there is a threat of collision, driver 2 applies corrective actions using decision making mechanisms to identify those rules that are relevant to their situation, and to assess the importance of each relevant rule. Appropriate driving actions are applied, e.g. slow down, check lane change, steer and move applying speed change and direction change.

As shown in figure 5.12 (b), driver 2 decides to overtake because his preferred forward distance is infringed. In the case of no threat of collision, driver 2 will continue to track driver 1 at each time step until driver 1 is no longer within the field of view or out of driver 2's visual range. 
Table 5.1 illustrates the result for unsighted driver 2 in scenario 1 . In the $8^{\text {th }}$ time step (in the time column of the table 5.1) both drivers are occupying lane 1. However, driver 2 decides to move to lane 2 in the $10^{\text {th }}$ time step, whilst driver 1 continues to stay in lane 1 through out the simulation. More detail discussion of the distance and speed perception is given in section 5.3.3.2.

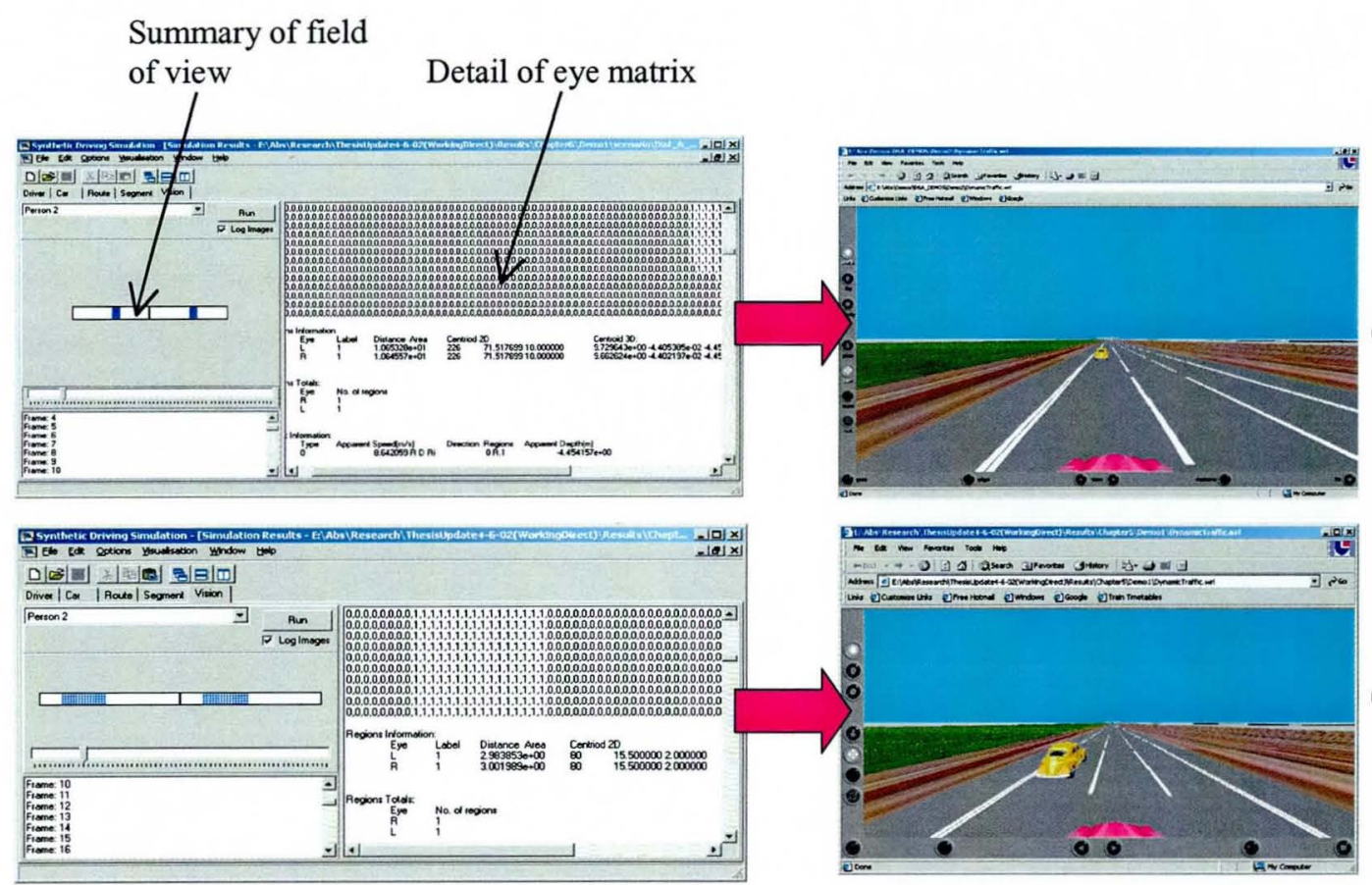

Figure 5.12: Seeing and making sense of visual information: (a) image capture at 8 seconds (b) image capture at 10 seconds

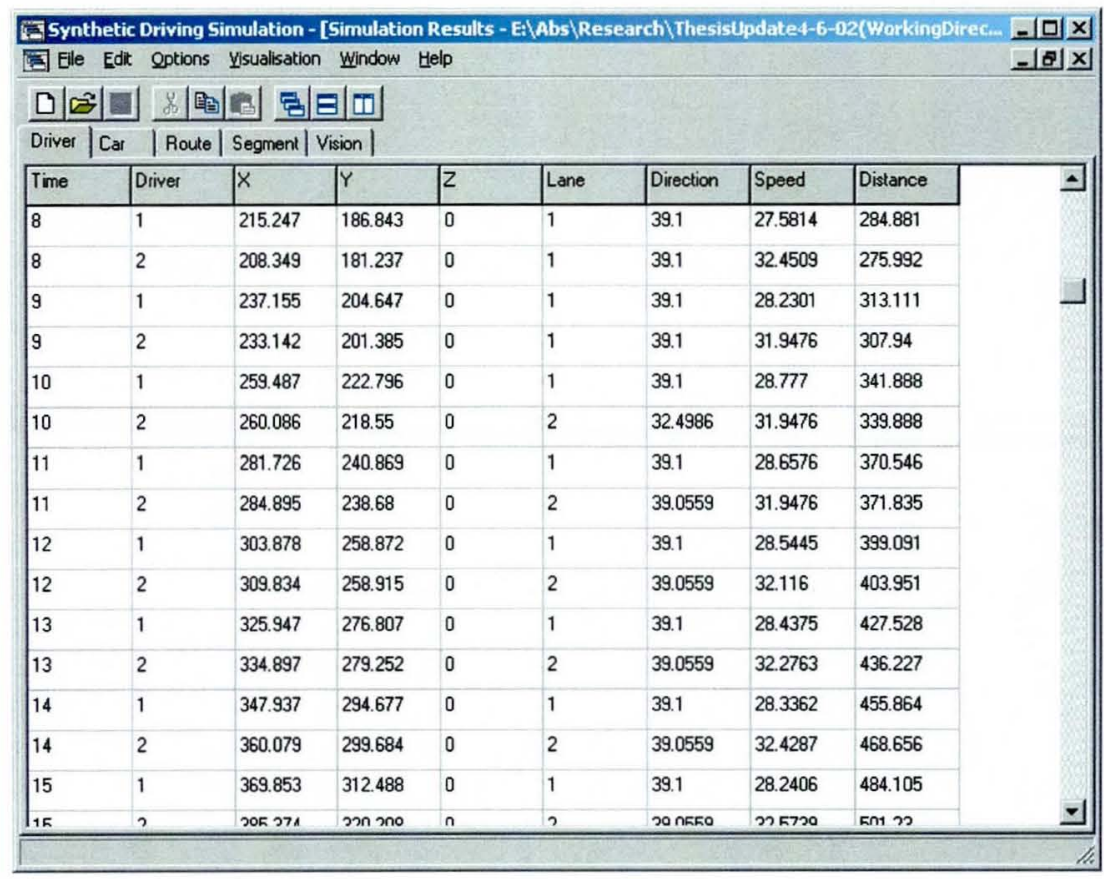




\subsubsection{Distance and Speed Perception}

Tables 5.2 and 5.3 illustrate distance and speed perception for unsighted and sighted driver 2 in the first scenario. Sighted driver agents refer to those with vision enabled capability and unsighted drivers employ perfect knowledge calculations.

\begin{tabular}{l|ccccc}
\hline $\begin{array}{l}\text { Type of } \\
\text { driver }\end{array}$ & $\begin{array}{l}\text { Time } \\
\text { steps (s) }\end{array}$ & $\begin{array}{l}\text { Preferred } \\
\text { front dist. } \\
(\mathrm{m})\end{array}$ & $\begin{array}{l}\text { Calculated } \\
\text { relative } \\
\text { front dist.(m) }\end{array}$ & $\begin{array}{l}\text { Calculated } \\
\text { relative } \\
\text { spd.(m/s) }\end{array}$ & $\begin{array}{l}\text { Image } \\
\text { area }\end{array}$ \\
\hline \multirow{5}{*}{ Unsighted } & 0 & 40 & 69.700 & 0.000 & -- \\
& 1 & 40 & 60.700 & 9.000 & -- \\
& 2 & 40 & 51.700 & 9.000 & -- \\
& 3 & 40 & 42.699 & 9.000 & -- \\
& 5 & 40 & 34.429 & 8.270 & -- \\
& 6 & 40 & 26.865 & 7.565 & -- \\
& 7 & 40 & 19.983 & 6.882 & -- \\
& 8 & 40 & 13.758 & 6.224 & -- \\
& 9 & 40 & 8.889 & 4.870 & -- \\
\hline \hline
\end{tabular}

Table 5.2: Unsighted (Perfect knowledge) calculations of distance and speed 


\begin{tabular}{|c|c|c|c|c|c|c|c|c|c|}
\hline \multirow{3}{*}{ Type of driver } & \multirow{3}{*}{ Time steps (s) } & \multirow{3}{*}{$\begin{array}{l}\text { Preferred front } \\
\text { dist. (m) }\end{array}$} & \multicolumn{7}{|c|}{ Vision model estimates of distance and speed } \\
\hline & & & \multicolumn{2}{|c|}{ Left eye } & \multicolumn{2}{|c|}{ Right eye } & \multirow{2}{*}{$\begin{array}{l}\text { Stereo depth } \\
\text { or } L_{\text {depth }}\end{array}$} & \multirow{2}{*}{$\begin{array}{l}\text { Total Area } \\
\text { occupied by } \\
\text { image in both } \\
\text { eyes }\end{array}$} & \multirow{2}{*}{$\begin{array}{l}\% \text { Occupied } \\
\text { by FOV for } \\
\text { both eyes }\end{array}$} \\
\hline & & & $\begin{array}{l}\text { Relative front } \\
\text { dist. (m) }\end{array}$ & $\begin{array}{l}\text { Relative front } \\
\mathrm{spd}(\mathrm{m} / \mathrm{s})\end{array}$ & $\begin{array}{l}\text { Relative front } \\
\text { dist. (m) }\end{array}$ & $\begin{array}{l}\text { Relative front } \\
\mathrm{spd}(\mathrm{m} / \mathrm{s})\end{array}$ & & & \\
\hline \multirow{10}{*}{ Sighted } & 0 & 40 & 68.634 & 0.000 & 68.632 & 0.000 & 68.581 & 20 & 8.0 \\
\hline & 1 & 40 & 59.716 & 8.860 & 59.714 & 8.983 & 59.663 & 24 & 9.6 \\
\hline & 2 & 40 & 50.761 & 8.894 & 50.759 & 9.017 & 50.708 & 36 & 14.4 \\
\hline & 3 & 40 & 41.704 & 9.015 & 41.713 & 9.125 & 41.657 & 54 & 21.6 \\
\hline & 4 & 40 & 33.556 & 8.100 & 33.561 & 8.205 & 33.506 & 92 & 36.8 \\
\hline & 5 & 40 & 25.953 & 7.558 & 25.960 & 7.664 & 25.904 & 130 & 52.0 \\
\hline & 6 & 40 & 18.984 & 6.920 & 18.982 & 7.033 & 18.931 & 170 & 68.0 \\
\hline & 7 & 40 & 12.821 & 6.102 & 12.819 & 6.225 & 12.768 & 180 & 72.0 \\
\hline & 8 & 40 & 8.028 & 4.733 & 8.024 & 4.857 & 7.974 & 200 & 80.0 \\
\hline & 9 & 40 & 4.320 & 3.645 & 4.316 & 3.771 & 4.266 & 210 & 84.0 \\
\hline
\end{tabular}

Table 5.3: Driver 2 perceives driver 1 - distance and speed estimates for sighted driver 
For unsighted driver 2, the calculated relative distances and speeds between driver 2 and 1 shown in table 5.2 are decreasing in each time step. This is due to the fact that driver 1 is travelling slower than driver 2. Driver 2's calculations of driver 1's distances and speeds takes into account explicit knowledge of driver 1's position and speed at each time step. The last column in table 5.2 indicates driver 2 has no access to image information since this is not available for unsighted drivers. However in table 5.3 driver 2 now sighted has to make subjective estimations of driver 1's distances and speeds. The distance and speed values for both the left and right eyes are again decreasing, consistent with those in table 5.2.

It is important to note that the distance and speed values estimated by both eyes are different. This is because as driver 1's vehicle moves within driver 2's field of view (FOV), there may be more rays in one eye hitting the vehicle than the other eye, so the calculations actually takes into account this motion. Although the vehicle doesn't seem to appear within driver 2's stereo region (driver 1's vehicle is always a long distance away at each time step and outside the stereo range, the depth of the vehicle can be determined as the midpoint of the disparity between the two eyes, using the relation in the CreateObjectCue() algorithm. The values in the stereo column actually combined the left and right eye estimates of distance relative to the eye planes rather than the centre of the head (see figure 4.6).

The total area occupied by the image in each eye at each time step refers to the total number of 1's counted. For example, at time step 1s, the binary image registered in driver 2's left and right eye is 12 and 12 respectively, giving a total of area of 24 pixels. In table 5.3 the percent of image area that occupies the FOV is obtained by dividing the total image area with the total eye resolution or pixels. For example, scenario one was run with an eye resolution of 250 (i.e. 50 columns and 5 rows), therefore, in time $1 \mathrm{~s}, 24 \%$ of the image area actually occupies the field of view. As the image area grows bigger, the perception of area that occupies the FOV increases accordingly. The importance of image area relating to the evaluation of direction of motion is investigated in section 5.3.3.3. The influence of endowing virtual driver agents with vision capabilities is observed in figure 5.13 which compares distance and speed perception for sighted and unsighted drivers. The figure shows that sighted drivers estimate of distance and speed is close to unsighted drivers or drivers with 
perfect global vision. In other words sighted driver's decisions are more realistic since they now have to make subjective estimate of other vehicles positions and speeds through SEE rather than rely on perfect global vision.

Comparing driver 2's perfect knowledge calculations (unsighted) and subjective estimates (sighted), it appears driver 2 is consistently underestimating both distance and speed at each time step. This is mainly due to the design of SEE. Since SEE's image capturing functionality is based on ray tracing, each ray is clipped when it intersect with an object face so that the total mean distance of all the rays represent the depth of the nearest face to the driver. For example, the following driver's estimate of the lead vehicle's distance is measured from the back face of the lead vehicle. In the unsighted case the vehicle of the lead vehicle is treated as a point mass.

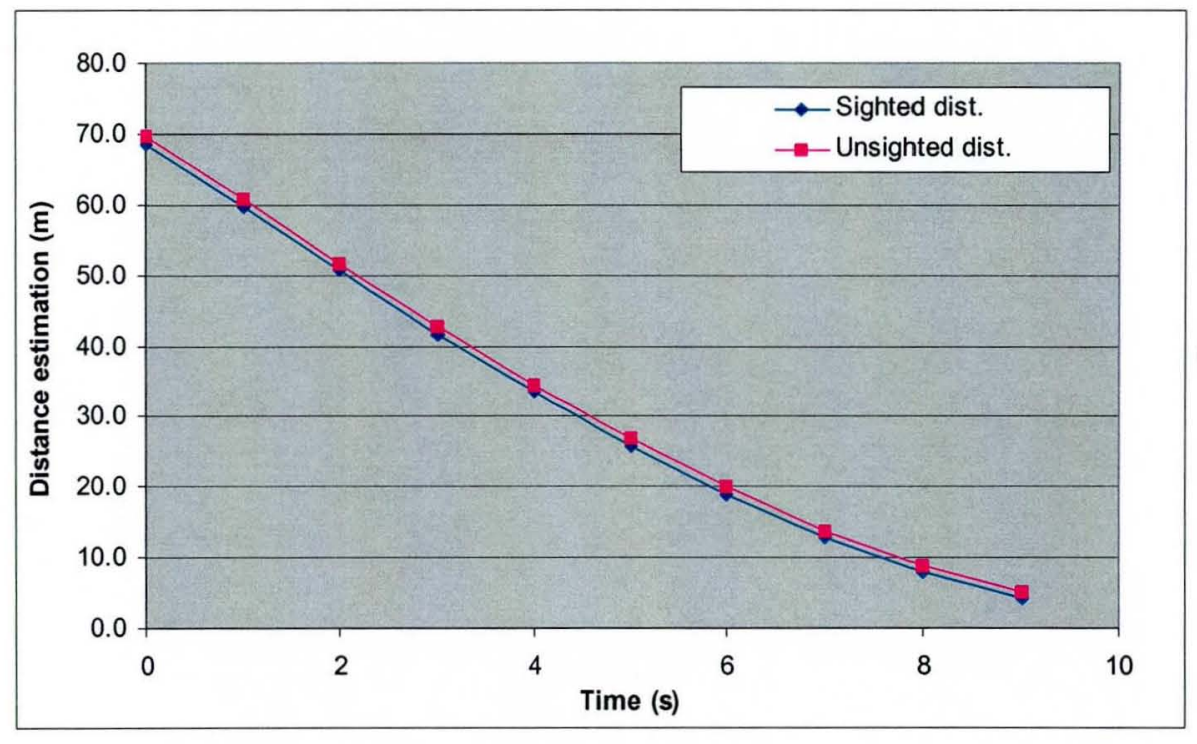

(a)

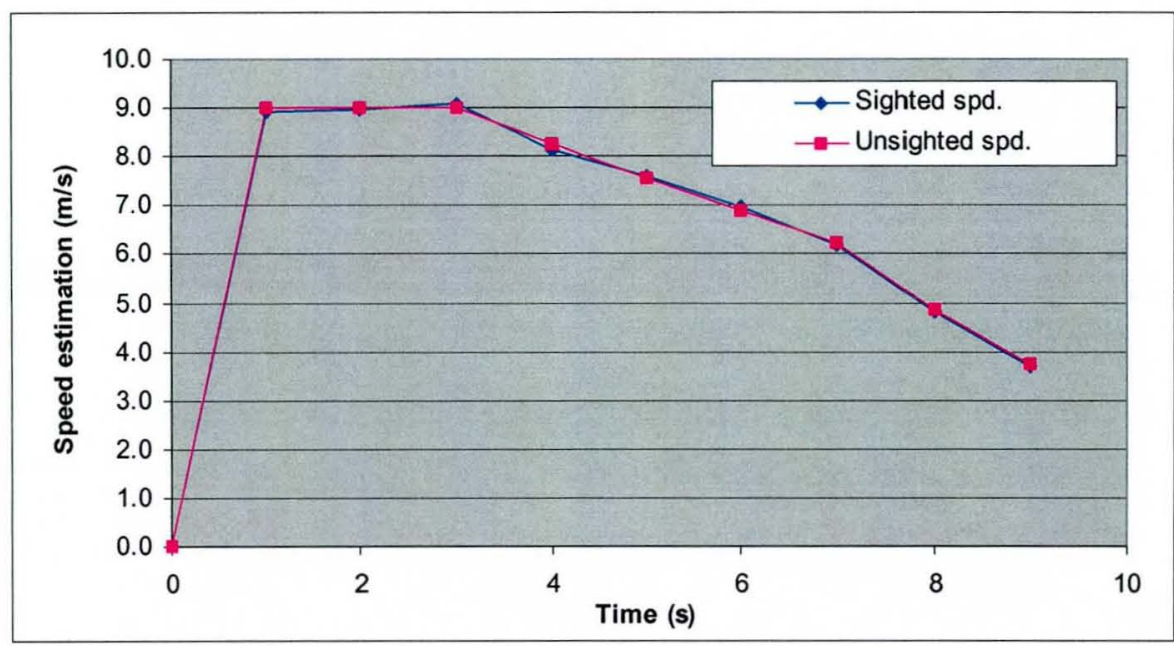

(b)

Figure 5.13 Comparison of (a) distance and (b) speed estimation for sighted and unsighted driver agent in scenario 1 


\subsubsection{Motion and Looming Detection}

As explained in chapter 4 the image area registered in the eyes is inversely proportional to the perceived distance, such that, as the image area expands, the distance between the two vehicles decreases. This expanding and contracting of image area due to looming and receding of objects can also provide direction of motion and time to collision information. Motion analysis in terms of looming vehicle from sighted driver 2's point of view is demonstrated for the first scenario, described in section 5.3.3.1. Figure 5.14 shows that as driver 2 increases his speed, he approaches closer to driver 1 who is travelling relatively slower. This triggers more rays in driver 2's eyes, leading him to start slowing down.

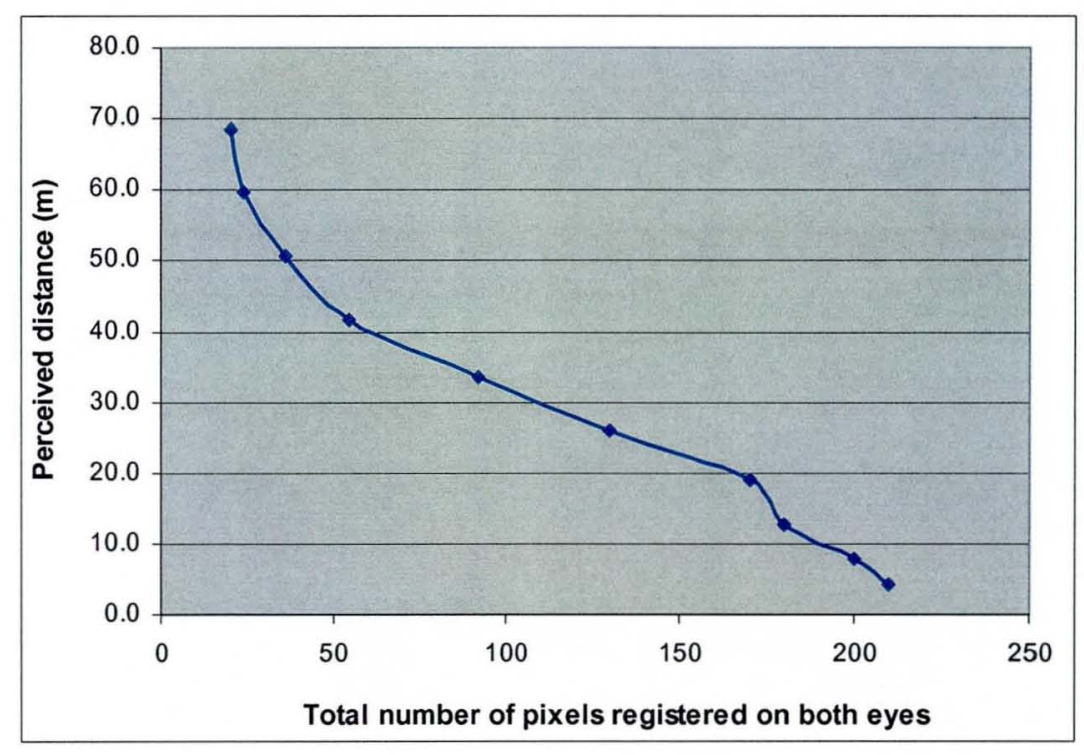

Figure 5.14 Image looming detection

\subsubsection{Car Following}

The current implementation of SD-SIM does not readily support visualisation of decision making relating to car following and lane changing. This is because decision making is an internal process performed by each driver agent. The outcome of decisions is a result of ranking all the rules in a decreasing order. As such it would require explicit representation of internal parameters for each decision taken in each time step. This poses several difficulties e.g. access to internal parameters during simulation to evaluate the combination of rules that have led to an outcome. Also, physically, selecting appropriate parameters to configure, debug and visualise decisions is time consuming. In short, virtual driver agents have a 'mind of their own' and it is not always easy to tell what they are 'thinking'. 
However, at the end of the simulation it is possible to visualise the consequences of all decisions made. For example, drivers have preferences such as the need to maintain minimum headway, maintain position within lane, etc. and react when these preferences are infringed. Therefore, one indication of driver decisions is to infer from their reaction or behaviour. In addition the internal processes of car following and lane changing can be demonstrated in pseudo code implementations. This is illustrated in figure 5.15 .

Virtual driver agents in SD-SIM use capabilities provided in the implementation of looming detection, along with perception of distance and speed to perform car following and lane changing. Car following occurs when a particular driver detects and starts to process visual information (such as relative distance, relative speed, distance headway) of the current lead vehicle that poses a threat of collision. The threat of collision or the need for safety is generated if the looming image area, A registered on the retina grows to provide time to collision information (see figure 5.14 and section 6.2.2) and the perceived relative distance and speed (of the detected vehicle) falls below the driver's preferred (or desired) distance and speed. This will lead to either a slowing down or lane change to avoid the collision. In the case where a lane changing manoeuvre is not possible due to other vehicles occupying the target lane, the following driver will adjust his speed to maintain a minimum constant preferred headway and follow the lead vehicle.

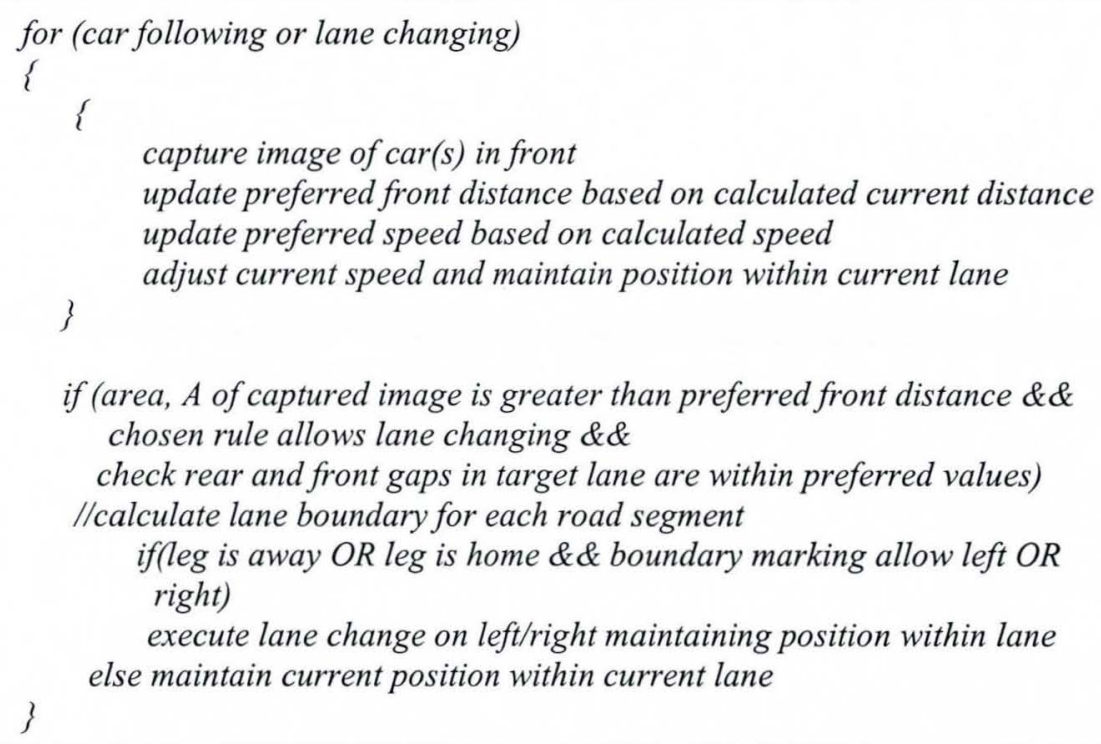

Figure 5 15: Qualitative visualisation of car following and lane changing decisions 


\subsubsection{Lane Changing}

As shown in figure 5.15, lane changing is triggered by three conditions (1) if the perceived separation distance (distance headway) and speed between the following driver and lead vehicle are below the following driver's preferred front distance and speed, (2) if the chosen rule (e.g. change lane) allows the following driver to overtake the lead vehicle and (3) if a suitable gap exists in the target lane, then the following driver will execute a lane changing behaviour. The decision to change lane due to chosen rule and gap acceptance conditions are very important and depend on the configuration of the rule weightings. At the same time, the rate of looming or receding image area is significant in assessing a suitable gap in the proposed lane. For example, an aggressive driver will decide to accept a shorter gap or minimum headway and choose to speed up in order to overtake.

Lane changing in SD-SIM is not explicitly programmed in the software but emerges as consequence of driver agent interactions. To analyse such interactions, the driver agents' lead gap (since drivers only have forward vision) acceptance behaviour is considered. This gap is measured as the distance headway between two vehicles. The setup for this scenario was described in section 5.3.3.1 as scenario two. Table 5.4 shows the lead gaps between drivers 2 and 1 relative to driver 2 (D2»D1), drivers 2 and 3 relative to driver $2(\mathrm{D} 2 » \mathrm{D} 3)$, and drivers 1 and 3 relative to driver $1(\mathrm{D} 1 » \mathrm{D} 3)$ during the first 11 time steps of the simulation. The table also compares the ability of sighted and unsighted drivers to accept suitable gaps.

Figure 5.16 shows lane occupancy and distance covered as implicit function of time. Note that the time domain is implicit in these graphs. From the graph (a) and table 5.3 , it can be seen that unsighted drivers 1 and 3 initiated a lane change to lanes 1 and 2 respectively in the second time step $(\mathrm{t}=1)$. This is because the lead gap between driver 1 and $3(\mathrm{D} 1 » \mathrm{D} 3)$ when driver 3 decided to change to lane 2 was below driver 1 's preferred front distance [i.e. at $\mathrm{t}=1,4.154<20$ ] so driver 1 had to move to lane 1 . However, the interaction between driver 2 and driver $1(\mathrm{D} 2 » \mathrm{D} 1)$ when driver 1 moved to lane 1 at this time step is not affected since the gap between them is greater than driver 2's preferred front distance [i.e. at $t=1,46.070>20$ ]. Driver 2 does eventually change to lane 2 but this does not have much effect on driver 3 since driver 3 was 
already in front of driver 2. They were also travelling at the same speeds and the gap between them was constant (D2»D3). The same behaviour is experienced by sighted drivers (figure 5.16b), although with slight underestimation of lead gaps.

\begin{tabular}{|c|c|c|c|c|c|c|}
\hline \multirow{2}{*}{$\begin{array}{c}\text { Time } \\
\text { step (s) }\end{array}$} & \multicolumn{5}{|c|}{ Lead Gap Acceptance - Distance (m) } \\
\cline { 2 - 7 } & \multicolumn{3}{|c|}{ Unsighted driver } & \multicolumn{3}{c|}{ Sighted driver } \\
\cline { 2 - 7 } & D2»D1 & D2»D3 & D1»D3 & D2»D1 & D2»D3 & D1»D3 \\
\hline \hline 0 & 51.282 & 50.423 & 0.859 & 50.325 & 49.844 & 3.013 \\
1 & 46.070 & 50.225 & 4.154 & 45.189 & 49.377 & 4.727 \\
2 & 41.070 & 50.225 & 9.155 & 40.204 & 49.398 & 8.956 \\
3 & 36.070 & 50.225 & 14.155 & 35.195 & 49.418 & 13.787 \\
4 & 31.070 & 50.224 & 19.155 & 30.143 & 49.438 & 18.618 \\
5 & 26.069 & 50.224 & 24.155 & 25.200 & 49.458 & 23.577 \\
6 & 21.143 & 50.297 & 29.155 & 20.585 & 49.370 & 28.492 \\
7 & 16.143 & 50.298 & 34.155 & 15.703 & 49.484 & 33.641 \\
8 & 11.143 & 50.298 & 39.155 & 10.858 & 49.500 & 38.439 \\
9 & 6.143 & 50.298 & 44.155 & 6.319 & 49.404 & 43.604 \\
10 & 1.143 & 50.298 & 49.154 & 2.818 & 49.311 & 48.456 \\
\hline
\end{tabular}

Table 5.4: Gap acceptance values during lane changing for unsighted and sighted driver 

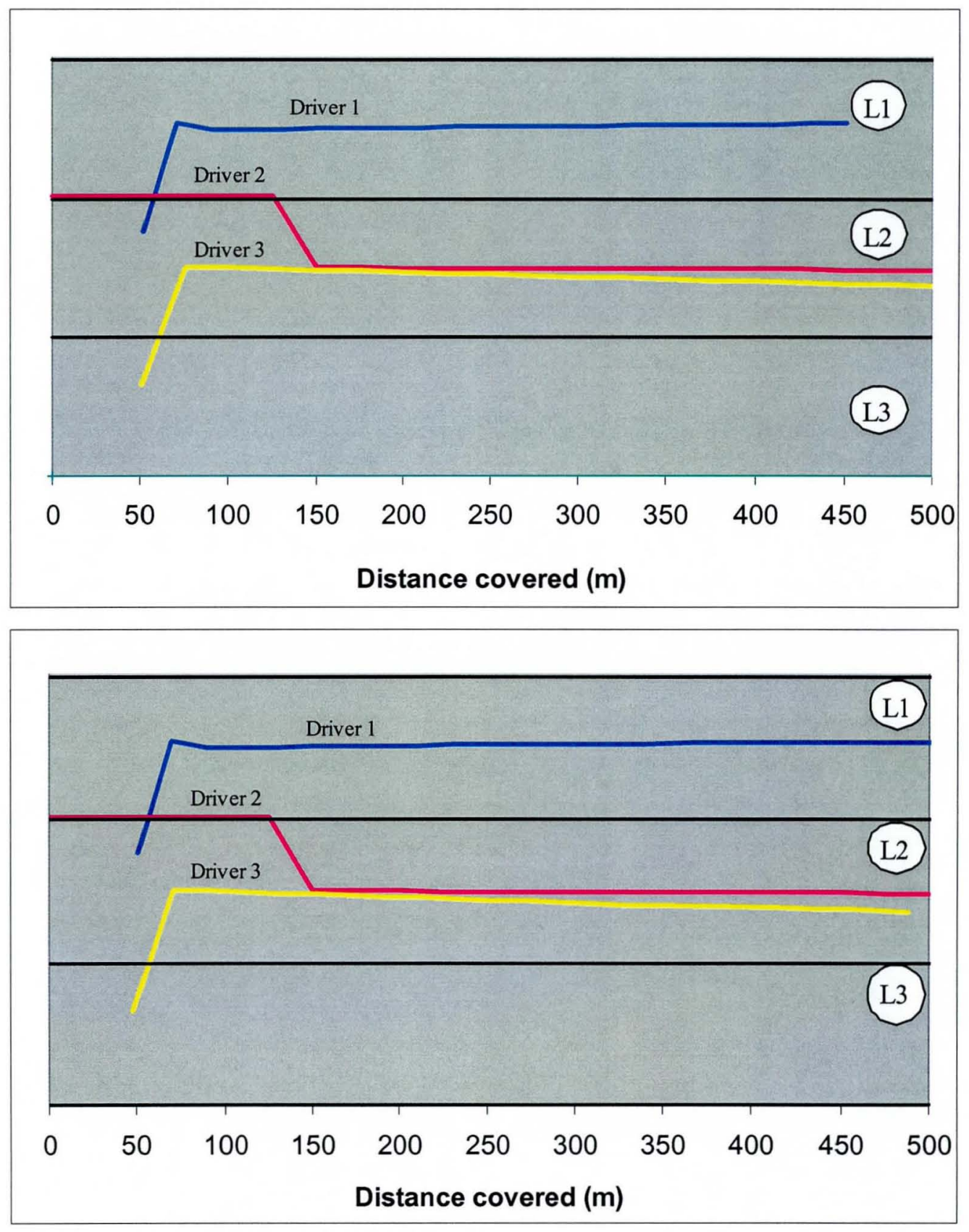

Figure 5.16 Lane position profiles during lane changing behaviour for (a) unsighted (b) sighted driver

\subsubsection{Execute Action}

The control of vehicle movement in SD-SIM is implemented as a direct response of the action executed by a virtual driver agent. The decision making mechanism calls the action module to take appropriate actions. The execute action algorithms are implemented for vehicle control commands such as to brake, accelerate, gear change 
and steer. The brake action is passed the calculated brake fraction, the current time step and the lane number to reduce the current driver's speed. In the case of acceleration, the calculated accelerator fraction along with the current time step and lane number is used to increase the current speed of the vehicle. The gear change action also limits the capacity of the vehicle to accelerate. The steering action receives parameters including time step, lane, a pointer to the current segment and current node of the road. The driver agent uses the steering and other signals (e.g. direction of the road, target position on the segment) to control the vehicle's orientation and heading. At each time step in the simulation, the status of the vehicle is then updated as a result of driver decision making.

Although SD-SIM does not provide explicit visualisation for execute action, the effect of actions taken by individual driver agents can be observed from the animation of calculated behaviour data. Scenario 1, described in section 5.3.3.1 is used here to illustrate execute action. Figure 5.17 shows qualitative visualisation of driver 2's steering action. In this scenario, driver 2 was programmed with a preferred lane that is lane 1 , so once driver 2 deems it safe to do so he moves back to his preferred lane, adjusting his speed and maintaining a safe rear distance. However it is noticeable that driver 2 over compensates his steering to the left and back to the right trying to correct his steering to position his vehicle's orientation. In fact such behaviour is common with human drivers who typically exhibit problems of vehicle control and orientation especially after lane changing manoeuvres (Wallis et al 2000).
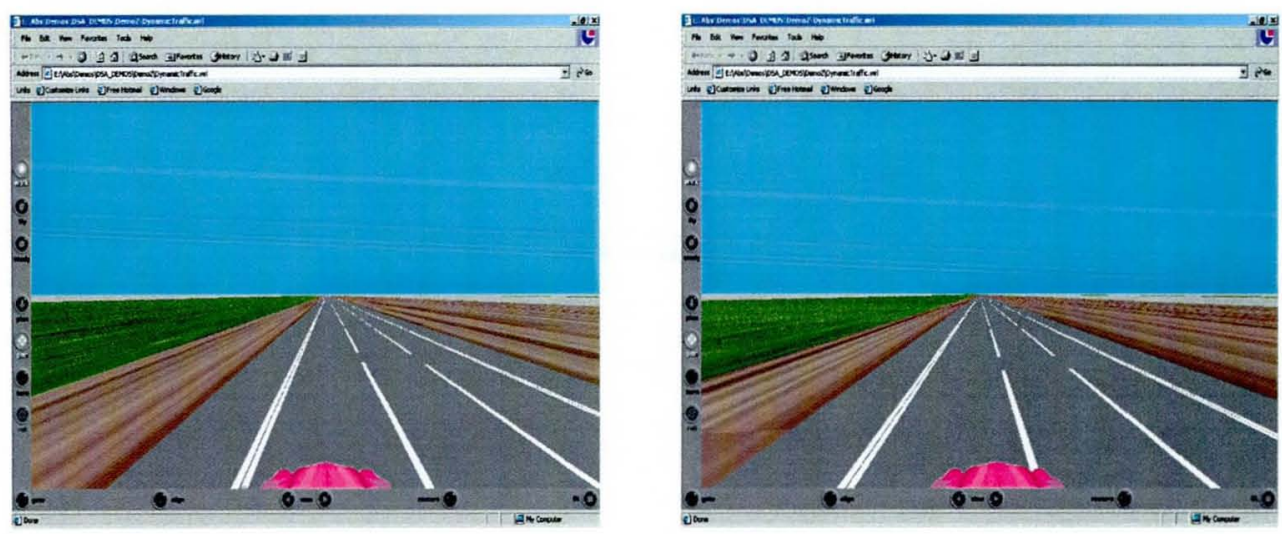

Figure 5.17: Over and under steer 


\subsection{Implementation Tools}

To end this chapter, it is worth discussing some of the most relevant tools and techniques that have influenced the development of SD-SIM. In this respect, hardware, software considerations and general system performance are also discussed.

There is a diversity of implementation tools and techniques that are useful in the modelling, simulation, animation and user interface development. SD-SIM is implemented using a suite of $\mathrm{C} / \mathrm{C}$ development tools. The decision making was initially developed using a $\mathrm{C}++$ procedural approach (Arnold 1997), whilst the vision model was implemented in C (Dumbuya 1999). To allow compatibility between these different modules, it was decided to merge these modules within an integrated framework now known as SD-SIM. The earlier stages of development of SD-SIM were considered in Microsoft ${ }^{\circledR}$ Visual $\mathrm{C}++$ development environment. This environment includes a source code editor, compiler and debugger. However, Microsoft Visual C++ did not provide the flexibility needed to develop SD-SIM's overall graphical user interface and therefore the final stages of SD-SIM's development especially its user interface were achieved in the Borland $\circledast \mathrm{C}++$ Builder development platform. This was found to be a more intuitive development environment with significantly better features, facilities and robustness in user interface development.

Before implementing SD-SIM, the need to integrate the different modules required a unified object oriented software engineering effort. This was achieved using the standard Rational Rose ${ }^{\circledR}$ tool - the Unified Modelling Language (UML). UML allowed different classes of SD-SIM to be packaged into a class structure (see chapter 4). This class structure is extensible, meaning more classes can be added to build more complexity in the framework in the future.

To enable integration of different modules in SD-SIM, a number of software development technologies were considered. Some of these included Application Programming Interface (API), Dynamic Link Libraries (DLLs) and Component Object Model (COM). In addition, visualisation technologies such as Virtual Reality Modelling Language and OpenGL also influenced the choice of implementation. API 
provides the facility to exchange messages or data between different software applications typically through simple subroutine calls. Since, the decision making model was developed in $\mathrm{C}++$ and the vision model in $\mathrm{C}$, an ideal approach would have been to link the two applications using an API, e.g. through data sharing by formatted file transfer. However, the API approach was found not to be suitable in this case because it required a large investment of time in analyses, software engineering and re-write of individual application features. The author believed this was an unnecessary diversion of the integration process since individual applications had already been tested. Therefore, time spent in further analysis and software engineering would not have contributed much to the overall design and implementation of the unified framework.

The use of DLL technology for such a large integration project offered many advantages over API. Dynamic linked libraries are much simpler to implement and save memory and disk space since functions can simultaneously share a single instance of a DLL in memory. Furthermore, DLLs support programs developed in different languages to share the same DLL through a defined function calling convention. However, the use of DLLs only offers the means of structuring the functionality into a set of API functions. Often, especially in simulation development, information also has to be structured, for example to distinguish a driver from a car. Also maintaining the decision making and vision model as two separate applications without a proper 'linking' mechanism will cause synchronisation problems. Therefore, DLL technology needed to be supported by Component Object Model (COM) technology.

A more advanced and robust implementation technology suitable for the integration of SD-SIM's different modules is COM. The creation of software components is a very efficient way to integrate different pieces of software while maintaining a distinct separation between functional modules. In this case, the software components are reusable, self-contained code modules that can be easily 'wired' together with other applications. Component Object Model is an object oriented windows based programming technology and contains two types of applications, servers and clients. A server is essentially an executable DLL or EXE application which supplies data, whilst a client application requires data. The communication between a client and 
server is achieved through the COM interfaces. A COM interface supports "wraps" of different pieces of code (developed in different language, style and platform), exposing only those interfaces required by the client and hiding the details of the implementation of the server. SD-SIM is implemented as COM with wrapper classes allowing only essential interfaces to be exposed to support the integration between the various modules (e.g. vision model, driver decision model, execute action) with the graphical user interface and underlying program functionality. This ensures that the different modules of SD-SIM are seamlessly integrated, with minimal modifications and maximum compatibility with existing code.

\subsubsection{Hardware and Software Considerations}

SD-SIM has been implemented to run on a desktop personal computer (PC) with considerations to minimum system requirements including:

- PC Hardware/Win 32 Platform, with Microsoft® Windows@ 98/2000/NT

- Pentium ${ }^{\circledR} 166 \mathrm{MHz}$ or higher is recommended

- Display monitor $-800 \times 600$ high colour mode is recommended

- OpenGL graphics accelerator

- Interface - Keyboard and mouse

- Random Access Memory (RAM) of 128 MB minimum

- Microsoft Internet Explorer 4 or higher

\subsubsection{System Performance}

Although, section 5.4.1 provides minimum system requirements, the selection of hardware resources and switching on certain simulation features has a great impact on the performance of SD-SIM. For example, running SD-SIM for 60 time steps, on a Pentium, P133, with 128MB of RAM, and three drivers each given an eye resolution $500 \times 500$ took $3.87 \mathrm{hrs}$ calculation time to step through 60 seconds. There are a number of ways of improving the performance of SD-SIM for example using parallel computing so that different components of the software (e.g. vision calculations, decision making calculations, visualisation, etc.) can run asynchronous processes on different processors. Another possible alternative approach is to allow different components of the program to be switched on or off depending on the user's interest. 
This is what has been implemented in SD-SIM, for example turning off the vision model from time to time to speed up simulation execution. Instead of switching off visual capabilities, a lower eye resolution can alternatively be defined for individual virtual driver agents.

\subsection{Chapter Summary}

This chapter has presented the implementation of SD-SIM, demonstrating key features and functionality. Visualisation is an important issue to the user and typically forms part of the initial validation of complex systems such as driving simulation. However, there are few tools available that provide all the facilities required to debug, analyse and understand the results of a driving simulation. The implementation of SDSIM in terms of its user interface and its suite of features allows qualitative and quantitative visualisation of traffic flow interactions. Qualitative visualisation in SDSIM is achieved by graphical and animation techniques, whilst quantitative visualisation is provided for by displaying calculations in information boxes or writing results to $\log$ files. This enables driver profiles to be created using Excel spreadsheets.

This chapter has discussed some specific driver behaviour such as object detection, car following, lane changing, gap acceptance, overtaking. Demonstrated simulation results are obtained from suitably designed scenarios to demonstrate the capabilities of some of the algorithms implemented within SD-SIM. Certain behaviour, such as car following and lane changing, is not easily quantified, since access to internal parameters during simulation is usually a difficult process. Furthermore, SD-SIM doesn't specifically model composite behaviour such as car following, overtaking, but instead enable drivers to perceive their environment, make decisions based on what they see and take appropriate actions. This leads to composite behaviour emerging as a consequence of driver interactions. This aspect of autonomous behaviour and emergent traffic flow forms the major contribution of the development of SD-SIM. Having discussed implementation issues here, chapter 6 discusses the quantitative performance of SD-SM. 


\section{CHAPTER 6}

\section{ALGORITHM PERFORMANCE MEASURES}

\subsection{Introduction}

This chapter assesses the quantitative performance of the Scene En-capturing and Evaluation (SEE) and the Intelligent Virtual Driver (IVD) modules within SD-SIM. Particular emphasis is placed on assessment of object detection, distance and speed perception, lane changing and collision avoidance. This assessment has been carried out in two ways:

- Comparison with previously published results for the modelling of visual perception. This is concerned with relating the performance of the SEE and IVD modules to studies involving specific decision-making based on optical flow information, such as time to collision and driving headways.

- Comparison with measured data. Experiments have been conducted, using accurate Home Office equipment for distance and speed measurement provided by Leicestershire Constabulary. With the help of Police drivers and other volunteers, various driving scenarios were defined and enacted within these experiments. These scenarios were primarily intended to generate accurate measurements in known circumstances. Of secondary importance, the opportunity was also taken to obtain data on the performance of the Police drivers and volunteers within these scenarios. Whilst these secondary results are useful and relevant, it should be noted that the experiments were not intended to be statistically significant and their discussion here should not be interpreted as a statistically sound analysis of driver perception.

Section 6.4 provides a simple case study to validate the contribution of SD-SIM to real-life scenario modelling. 


\subsection{Comparative Studies of Perceptual Factors}

In providing a basis for comparative studies, a brief review of the relevant literature is required to extract useful data to configure and assess SD-SIM's performance in terms of measures for optical flow information, driving headways and time to collision (TTC). To retain comparability between data, some assumptions e.g. simulation time steps and simulation durations have been made in calibrating SD-SIM. This comparative evaluation of SD-SIM's perceptual factors with related research is demonstrated in some cases for sighted and unsighted driver agents.

\subsubsection{Optical Flow Information}

In driving, human drivers are capable of tracking objects within their field of view and range to extract useful information about object motion relative to their own motion (optical flow information). There are two important measures associated with this, (1) focus of expansion (looming effect due to approaching an object) and (2) focus of contraction (receding effect). Each of these variables can be used to compute the distribution of the velocity field in the image and provide means of evaluating object motion e.g. time to collision or gap acceptance. Lee (1976), established a relationship between the visual angle $\alpha$ (in radians), subtended by an object and angular velocity of the object $\dot{\alpha}$ (radians per second), given in equations 6.1 and 6.2 (for very small $\dot{\alpha}$ ). These equations take into account the longitudinal (separation) distance $Z$ (in meters), velocity of locomotion (closing velocity) $V$ (in meters per second), and width of the lead vehicle, $W$ (in meters) as given by the expression in equation 6.1 .

$$
\alpha(t)=\frac{W}{Z(t)}
$$

The rate of change of visual angle is given as:

$$
\dot{\alpha}(t)=\frac{W V}{[Z(t)]^{2}}
$$


Several other studies (e.g. Schiff and Detwiler 1979, McLeod and Ross 1983, Groeger and Cavallo 1991) have performed experiments to analyse the use of optic flow information in making judgements e.g. during vehicle operation. However, these studies are limited to observers making judgments of time to collision whilst approaching a stationary lead vehicle. These experiments are performed in the laboratory with the subjects shown a film from the driver's view as the driver approached the stationary vehicle. The subjects then had to respond, usually by pressing a button when they thought collision with the stationary target vehicle will occur. Most of the studies reported that, drivers tend to use optic flow information in these situations with a general underestimation of time to collision.

The evaluation of optic flow information for sighted driver agents in SD-SIM is intended to support the use of such information to guide autonomous behaviour through visual perception of motion. Section 5.3.3.3 presented motion and looming detection example based on the relation $A \propto 1 / d$. This concept has been expanded to enable the assessment of SEE's performance against related research in optic flow field calculations during vehicle operation. Equation 6.3 is based on figure 6.1 and has been derived to account for the visual angle subtended by an object as a function of image flow. Similar assumptions to (Lee 1976) are adopted in figure 6.1, such that the driver agent and his eyes are moving in a linear motion (along the z-axis) through the traffic environment and images of the detected vehicle are captured in the horizontal field of view as shown in the figure $6.1 \mathrm{~b}$.

Further assumptions are, the eyes which are located on the head cannot move independently of the head. Images are captured on two $n \times m$ eye matrices, such that as the driver moves closer to the vehicle in front, the image area registered on the retina also increases, figure 6.1c. Also, the horizontal field of view given in chapter 4 is $\theta=120^{\circ}$ i.e. 2.09436 radians. From figure 6.1c, the visual angle as a function of image flow is given in equation 6.3.

$$
\frac{\alpha}{\theta}=\frac{\text { The longest run of } 1 \mathrm{~s}}{\text { Number of columns }}
$$




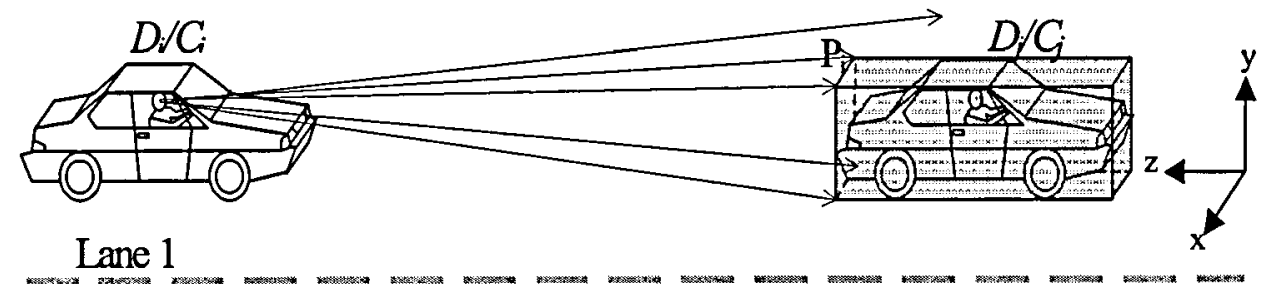

Lane 1

(a)
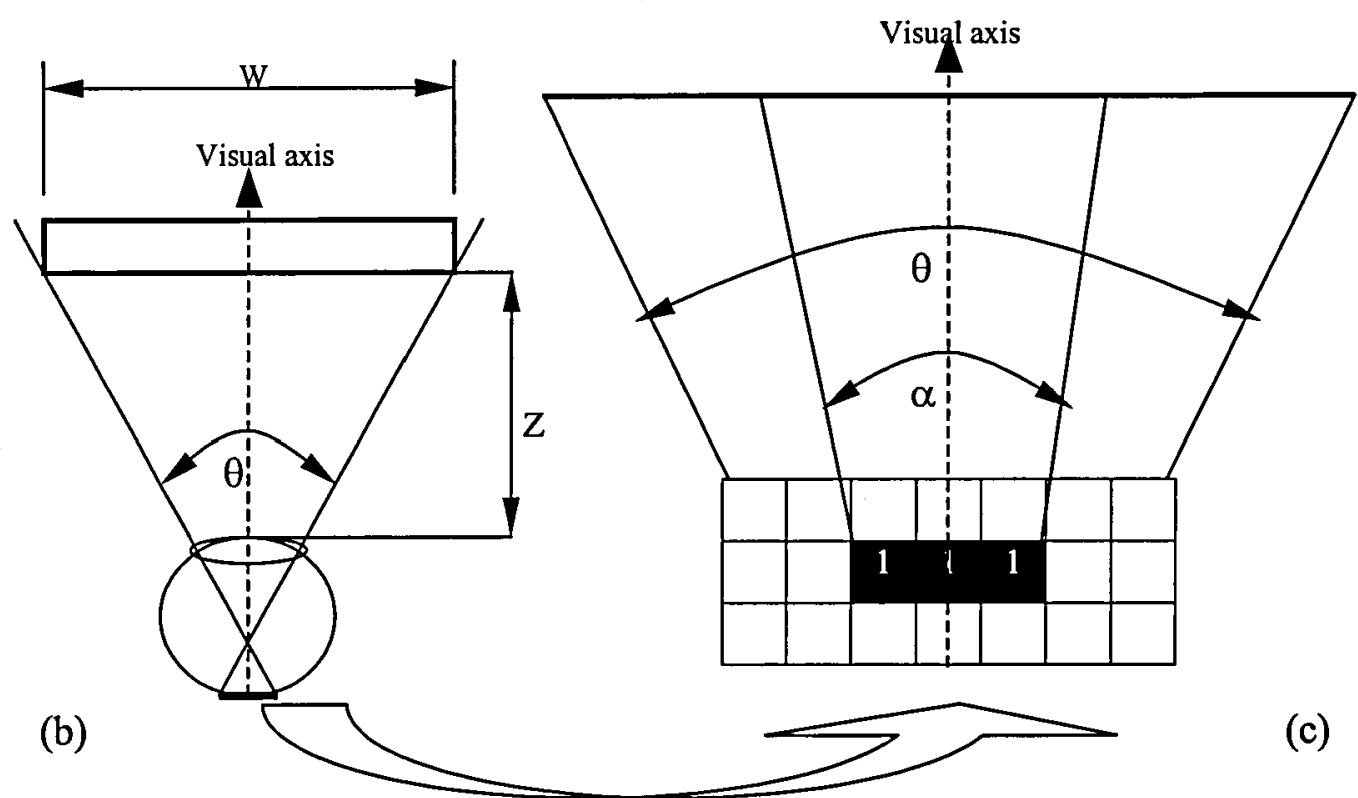

(c)

Figure 6.1 Optic flow for sighted driver agent (a) image capture from driver $D_{i}$ in car, $C_{i}(b)$ plan view from one eye (right eye) and (c) optic image formed in the $m \times n$ eye matrix

To determine the angular velocity $\varpi$ (in radians per second) of the retinal image, two consecutive frames are considered at a time such that the previous frame, $n-1$ is subtracted from the current frame, $n$ and divided by the difference between the previous time, $t_{n-1}$ and current time $t_{n}$, equation 6.4 .

$$
\varpi=\frac{\alpha_{n}-\alpha_{n-1}}{t_{n}-t_{n-1}}
$$

Applying these relationships to describe optic flow information due to driver agent's movements within the traffic environment, a scenario involving the 'stop and go' experiment described in sections 6.3.1.1 (experiment 1) has been configured within 
SD-SIM. This scenario was intended to compare results using equations 6.3 and 6.4 with equations 6.1 and 6.2. In this scenario the target vehicle was directly in front of a stationary observing virtual driver agent at an initial mean distance of $175.53 \mathrm{~m}$ (refer to table 6.6, column 2). The target object has a width of $1.8 \mathrm{~m}$. The eye resolution for the virtual driver agent was defined as 100 columns $x 100$ rows and 300 columns $x$ 100 rows in each run. The choice of eye resolution was based on improvements observed using higher eye resolution discussed in section 6.3.2.4.

The following visual variables; the visual angle $\alpha$ and angular velocity $\varpi$ were calibrated from the simulation data using the above equations. The comparison of outputs between (Lee 1976) and SEE is illustrated table 6.1. The time $0 \mathrm{~s}, 5 \mathrm{~s} 10 \mathrm{~s} 15 \mathrm{~s}$ and 20 s correspond to the intervals between image capture. It is obvious from the table that SEE is capable of registering visual variables such as visual angle and visual angular velocity. It is also noticeable that as the target object approaches the stationary observer, the visual angle subtended by the target object in the eyes increases. The angular velocity refers to the average velocity travelled by the target object between its discrete positions. The $20 \mathrm{~s}$ column in the table has no angular velocity value since speed was calculated between any two consecutive frames. The angular velocity is also shown to be increasing. There is no significant difference between 100 and 300 columns for both visual angle and angular velocity using Lee's equations. This is because there is only a small change in $Z$ calculated for 100 and 300 columns, see figure 6.10. However, there is some improvement in the comparison of visual angle and angular velocity for higher eye resolutions using SEE.

Considering table 6.1, the main source of errors in the calculation of optical flow information using Lee's equations 6.1, 6.2 and SEE's equations 6.3 and 6.4 is a result of the constancy of object width assumed in Lee's formulation. Although, SEE considers the physical object width to be constant, the image of the object registered on the eyes is not (see section chapter 5 - looming section). As the object approaches the observer, more rays are registered on the eyes, creating a larger image. In general, SEE's calculation of optical flow information using equations 6.3 and 6.4 assumes that the observer is detecting the object in stereo vision. When the object falls outside the stereo field e.g. in overtaking manoeuvres, the accuracy of SEE in calculating 
optical flow information based on the above equations is limited. This is because images are not properly connected since SEE currently relies on a 4-connnectivity algorithm (section 4.4.3.1) rather than a more robust 8-connectivity. 


\begin{tabular}{|c|c|c|c|c|c|c|c|c|c|c|c|c|c|c|c|c|c|c|c|c|}
\hline \multirow{4}{*}{$\begin{array}{l}\text { Number of } \\
\text { columns }\end{array}$} & \multicolumn{10}{|c|}{ Lee 1976} & \multicolumn{10}{|c|}{ SEE } \\
\hline & \multicolumn{5}{|c|}{$\left(\frac{W}{Z(t)}\right)$, radians } & \multicolumn{5}{|c|}{$\left(\frac{W V}{[Z(t)]^{2}}\right)$, radians per second } & \multicolumn{5}{|c|}{$(\alpha)$, radians } & \multicolumn{5}{|c|}{$(\varpi)$, radians per second } \\
\hline & \multicolumn{5}{|c|}{ Time (s) } & \multicolumn{5}{|c|}{ Time (s) } & \multicolumn{5}{|c|}{ Time (s) } & \multicolumn{5}{|c|}{ Time (s) } \\
\hline & 0 & 5 & 10 & 15 & 20 & 0 & 5 & 10 & 15 & 20 & 0 & 5 & 10 & 15 & 20 & 0 & 5 & 10 & 15 & 20 \\
\hline 100 & 0.010 & 0.012 & 0.015 & 0.021 & 0.034 & 0.000 & 0.001 & 0.002 & 0.004 & -- & 0.021 & 0.021 & 0.042 & 0.084 & 0.105 & 0.000 & 0.000 & 0.004 & 0.008 & -- \\
\hline 300 & 0.010 & 0.012 & 0.015 & 0.021 & 0.034 & 0.000 & 0.001 & 0.002 & 0.004 & - & 0.007 & 0.014 & 0.028 & 0.049 & 0.063 & 0.000 & 0.001 & 0.002 & 0.004 & -- \\
\hline
\end{tabular}

Table 6.1 Driver optic flow variables 


\subsubsection{Time To Collision}

Time to collision, TTC information plays a significant role in vision mediated behaviour e.g. collision avoidance and control of vehicle braking. TTC information can be derived from two sources (1) global information relating to distance and velocity, commonly referred to as cognitive or low order information and (2) local information also known as optic flow or high order information (McLeod and Ross 1983, Cavallo et al 1995). The definition of TTC relating to global information can be expressed by equation 6.5 and involves the driver estimating the distance to the lead vehicle with respect to his travelling velocity.

$$
T T C=\frac{d x}{d v}
$$

In this expression TTC, is the time (in seconds) taken for an observer (driver) travelling at constant speed to reach a specified point, $d x$ is the distance from the bumper of the following car to the rear bumper of the lead car (meters), and $d v$ is the difference between the speed of the following and the lead car (meters per second).

However, collision avoidance with stationary or moving vehicle requires the driver to anticipate danger and start slowing down (braking) to avoid impending collision. The information required for this kind of anticipation can be derived from optic flow information discussed in section 6.2.1. According to (Lee 1976), the relationship between the TTC and the expansion of the retinal image can be defined as the visual angle subtended by an object image over the rate of change of the visual angle at time $t$. This ratio is expressed in equation 6.6 and relates to local information derived from the environment.

$$
T T C=\frac{\alpha(t)}{\dot{\alpha}(t)}
$$

Where $\alpha(t)$ and $\dot{\alpha}(t)$ are the same as in equations 6.1 and 6.2. Extending equation 6.6 to include visual information or an optic variable that determines when a driver starts 
braking, Lee has defined a 'threshold time to collision variable' $\tau_{t h}$ (measured in seconds), given in equation 6.7 .

$$
\tau_{t h}=\left(\frac{W}{V \dot{\alpha}_{t h}}\right)^{1 / 2}
$$

Where $W$ is width of lead vehicle, $V$ closing (or relative) velocity and $\dot{\alpha}_{t h}$ threshold visual angular velocity which is assumed to be independent of the visual angle. This implies that drivers will start to break when they are at their threshold time to collision. Also, as noted in Lee's paper and obvious from the equation, faster closing speeds means shorter $\tau_{t h}$ and larger lead vehicle widths will result in longer $\tau_{t h}$.

Van Winsum and Heino (1996) provide a succinct review of studies that have shown consistent underestimation of TTC. In their review several papers (Schiff and Detwiler 1979, McLeod and Ross 1983, Groeger and Cavallo 1991) were identified. In general, the methodology adopted by the cited authors (except Schiff and Detwiler 1979) involved presenting subjects with video recordings (from the approaching vehicle's driver view) of an approach to a stationary target vehicle. The approach was filmed at various speeds and the subjects had to make estimates of when they thought collision with the target will occur. Schiff and Detwiler (1979) used animated table top photography as stimulus to the subjects. The authors observed an average underestimation of TTC of 42\% (McLeod and Ross 1983), 39\% (Schiff and Detwiler 1979 ) and 35\% (Cavallo et al 1986). Furthermore, similar experiments, but involving subjects actually driving the approach vehicle have been reported in (Hoffmann and Mortimer 1994). The authors reported underestimation of TTC by $20 \%$.

To compare TTC in SD-SIM with the previous results, SD-SIM was set up with two drivers for scenario 1 described in section 5.3.3.1. The simulation was run for 60 seconds in $1 \mathrm{~s}$ time steps and time to collision was calculated using equation 6.6. Table 6.2 shows comparable average underestimation of time to collision data calculated from this scenario using equation 6.5 . The value of $33.4 \%$ is within the 
literature bound and demonstrates that sighted driver agents in SD-SIM tended to underestimate time to collision information.

\begin{tabular}{|c|c|c|c|c|c|}
\cline { 2 - 6 } \multicolumn{1}{c|}{} & \multicolumn{5}{c|}{ Time to Collision (TTC) Studies } \\
\cline { 2 - 6 } & $\begin{array}{c}\text { Schiff and } \\
\text { Detwiler (1979) }\end{array}$ & $\begin{array}{c}\text { McLeod and } \\
\text { Ross (1983) }\end{array}$ & $\begin{array}{c}\text { Cavallo et al } \\
(1986)\end{array}$ & $\begin{array}{c}\text { Hoffmann and } \\
\text { Mortimer } \\
(1994)\end{array}$ & $\begin{array}{c}\text { Sighted virtual } \\
\text { drivers agents } \\
\text { [SD-SIM] }\end{array}$ \\
\hline Average TTC & $39 \%$ & $42 \%$ & $35 \%$ & $20 \%$ & $33.4 \%$ \\
\hline
\end{tabular}

Table 6.2 Comparison of TTC during car following

\subsubsection{Driving Headways}

Drivers' perception of headway in car following studies is usually expressed as time headway or distance headway (Fuller 1981). Time headway is defined as the time interval between two vehicles, equation 6.8 .

$$
t_{\text {headway }}=\frac{d x}{v}
$$

Where, $t_{\text {headway }}=$ is time headway (measured in seconds), $d x=$ distance from the bumper of the following vehicle to the rear bumper of the lead car in $\mathrm{m}$, and $v=$ the speed of the following vehicle measured in meters per second, $\mathrm{m} / \mathrm{s}$.

Distance headway, $d x$ can be derived from equation 6.8 as the bumper to bumper distance between the lead vehicle and following vehicle, expressed in meters. Alternatively, in (McDonald et al 1997), a more suitable parameter to describe the variability in driver headways is provided. This is expressed in the paper according to equation 6.9 .

$$
D S S D=\frac{D S}{S d}
$$

Where, DSSD is the Distance or Separation Divergence, $D S$ is the vehicle separation in meters, and $S d$ is the driver's desired or preferred following distance, meters. 
Time headway studies (e.g. van Winsum and Heino 1996, Fuller 1981, Taieb-Maimon and Shinar 2001) tend to measure time headways as the important variable in car following behaviour. In (van Winsum and Heino 1996), the authors measured time headways adopted by drivers in a driving simulator performing car following tasks and found that these headways are constant over different speeds, meaning the headways are independent of the vehicle speeds but vary between different drivers. An average preferred headway of $1.0 \mathrm{~s}$ was obtained in this experiment. The implication of time headway being independent of speed, seems to support the commonly recommended time headway given in driving manuals, for example, "keep at least two seconds behind the vehicle in front" (Lynch and Wood 1992).

Fuller (1981) studied the performance of truck drivers based on measurement of their time headways in a two vehicle convoy configuration ('continuous following'). The author observed that time headways adopted in this configuration are lower than in normal car following situation e.g. steady state following. Fuller reported mean time headways for different measures such as late shift drivers tended to have $1.93 \mathrm{~s}$ longer than early shift drivers $1.62 \mathrm{~s}$. However when the results were compared to previous experiments cited in the paper, the author noted that mean time headway for early shift drivers in the previous normal following experiment was $3.01 \mathrm{~s}$ compared to the new convoy experiment which was $1.62 \mathrm{~s}$. The general observation of Fuller's convoy experiments is that there was no general decline in performance due to prolonged driving. However, it should be noted that these experiments were conducted with military personnel who are used to such driving conditions and the results can therefore be questioned if they are representative of other drivers especially with contradiction with previous observations which reported 3.01s. Also, time headway can be associated with risky driving behaviour, keeping other factors constant. In other words, as noted in the paper, shorter time headways imply a generally higher level of risk taking. In fact the time headways adopted in the convoy experiments are lower than the recommended general $2 \mathrm{~s}$ rule. This could also imply, as some evidence has shown that the adoption of lower headways by drivers in the convoy experiments was because they were in fact compensating for performance abilities. Van Winsum and Heino (1996) provide a discussion of operational skills related to task and state factors which help to explain Fuller's findings. 
Taieb-Maimon and Shinar (2001) conducted real driving experiments to measure drivers' ability to maintain 'minimum safe distance' and estimate headways whilst involved in a car following situation. The results showed that the mean headway adopted by drivers was $0.66 \mathrm{~s}$ and that $90 \%$ of the subjects maintained a minimum safe headway of less than $1.0 \mathrm{~s}$. Comfortable headways were more than minimum headway, although less than the $2 \mathrm{~s}$ recommended headway. The discussion in the paper also confirmed previous findings of (van Winsum and Heino 1996) that drivers' adoption of time headways is independent of speed and consistent within individual drivers. In addition the authors noted the implication of the observation to drivers' ability to estimate time headway which is based on optic flow field rather than distance estimations.

As discussed above distance headway studies (e.g. Ohta 1993, McDonald et al 1997, Wu et al 2000) evaluate drivers' ability to maintain appropriate distance separations between themselves and the lead vehicle. Ohta conducted real traffic experiments to evaluate individual driver's differences in maintaining distance headways in car following situations using different speed ranges. The author classified general driving into three types based on his findings. Type 1 drivers tend to maintain a 'constant time headway', type 2 driver maintain 'a constant distance headway' and finally type 3 driver's distance headway maintenance is independent of the speed. Among these different types of drivers, time headway groupings were observed by the author; group 1 adopts less than $1.0 \mathrm{~s}$, group 2 ranged between $1.0 \mathrm{~s}$ and $1.6 \mathrm{~s}$ and for group 3 it was more than $1.6 \mathrm{~s}$. These findings are explained by the fact that some groups' maintenance of headway is influenced by social norms, (Ohta 1993) so that some drivers keep short headways as a way of conforming to the norm e.g. tailgating.

McDonald et al (1997) conducted experiments in an instrumented vehicle involved in car following and lane changing conditions on three lane British motorways. Subjects drove an instrumented vehicle and their perception of following distance and speed data was collected using verbalisation methods. The authors reported the desired distance headways obtained for the four driver subjects $(1,2,3$, and 4) as $0.61 \mathrm{~s}, 0.85$ $\mathrm{s}, 1.83 \mathrm{~s}$ and $0.86 \mathrm{~s}$. The variables, relative speed and closing distance (referred to in the paper as separation divergence, DSSD equation 6.9) were used to configure and calibrate rules in a fuzzy logic based driving simulation model. The same group, (Wu 
et al 2000) reported the mean preferred distance headway for six subjects ranging from minimum of $0.61 \mathrm{~s}$ and maximum of $1.92 \mathrm{~s}$. This disparity was suggested as a strong indication of the variation in drivers preferred headways for which the DSSD measure provides a better agreement between subjects and the empirical results $(\mathrm{Wu}$ et al 2000).

Given these findings SD-SIM was set up to evaluate virtual driver agent's estimate of time and distance headways using data from (Colbourn et al 1978). The choice of data from this paper was influenced by the fact that the paper addressed both distance and time headways using an experimental set up. The paper described an experiment conducted on a race track, involving subjects driving a test vehicle whilst following a lead vehicle driven by the experimenter. The study divided the subjects into three categories of inexperienced drivers, low experience drivers and experienced drivers. The experiments were conducted under these categories to measure subjects' headways in a car following situation involving a low probability that the lead car will slow down or stop and there was no possibility of overtaking the lead vehicle i.e. steady state following. This is termed in the paper as low $P$ condition 1 . Condition 2 with 'high probability' of the lead vehicle decelerating was termed high P condition 2. This section evaluates SD-SIM's driving headways based on low $P$ condition 1 for the different categories of drivers. This enables direct comparison of the results with those reported in the other distance headway literature discussed above.

In the configuration of SD-SIM the following variables and conditions have been considered:

- The lead vehicle speeds in SD-SIM were programmed with speed profiles of the lead vehicle described in (Colbourn et al 1978) i.e. 48.3, 66.0, 80.5, $\mathrm{km} / \mathrm{h}$ (approx. $13.42,18.33,22.36 \mathrm{~m} / \mathrm{s}$ respectively).

- The initial speeds of the following driver agent were also defined as $48.3,66.0$, $80.5, \mathrm{~km} / \mathrm{h}$ (approx. 13.42, 18.33, $22.36 \mathrm{~m} / \mathrm{s}$ ).

- The lead vehicle was placed in front of the following vehicle at an initial position corresponding to the mean separation distance in each speed range.

- The following drivers in SD-SIM were classified into inexperienced drivers, low experience drivers and experienced drivers. 
- The preferred following distance headway for each driver agent in each speed range was obtained by multiplying the mean time headway by that speed.

- The rules to maintain minimum headway, maintain position within lane were weighted for the following driver as 0.9 and 0.9 respectively i.e. higher values imply higher significance of the rule.

- Each driver agent was run as either sighted or unsighted. Sighted driver agents were given eye resolution of 100 columns by 50 rows. The choice of eye resolution was based on the need to balance out computational efficiency and adequate accuracy.

- The simulation was run for 80 time steps each of $1 \mathrm{~s}$ duration.

Applying equation 6.8 , table $6.3 \mathrm{a}, \mathrm{b}$ presents the mean distance in meters and time headways (time headways shown in parentheses in seconds) comparison between Colbourn's (Colbourn et al 1978) low $P$ condition 1 and unsighted and sighted driver agents in SD-SIM.

\begin{tabular}{lcccccc}
\hline & \multicolumn{3}{c}{$\begin{array}{c}\text { Mean distance and time headways } \\
\text { Colbourn et al study }\end{array}$} & \multicolumn{3}{c}{$\begin{array}{c}\text { Mean distance and time headways for } \\
\text { unsighted virtual driver agents }\end{array}$} \\
\hline Type of driver & $48.3 \mathrm{~km} / \mathrm{h}$ & $66.0 \mathrm{~km} / \mathrm{h}$ & $80.5 \mathrm{~km} / \mathrm{h}$ & $48.3 \mathrm{~km} / \mathrm{h}$ & $66.0 \mathrm{~km} / \mathrm{h}$ & $80.5 \mathrm{~km} / \mathrm{h}$ \\
\hline $\begin{array}{l}\text { Inexperienced } \\
\text { Low }\end{array}$ & $23.3(1.739)$ & $34.0(1.860)$ & $46.8(2.142)$ & $27.7(2.069)$ & $38.3(2.096)$ & $46.8(2.085)$ \\
experience & $23.9(1.780)$ & $32.8(1.822)$ & $44.7(2.038)$ & $28.3(2.114)$ & $41.4(2.264)$ & $44.7(1.996)$ \\
Experienced & $24.1(1.792)$ & $32.6(1.749)$ & $41.3(1.867)$ & $28.5(2.129)$ & $36.9(2.019)$ & $41.3(1.844)$ \\
\hline
\end{tabular}

(a)

\begin{tabular}{lcccccc}
\hline & \multicolumn{3}{c}{$\begin{array}{c}\text { Mean distance and time headways } \\
\text { Colbourn et al study }\end{array}$} & \multicolumn{2}{c}{$\begin{array}{c}\text { Mean distance and time headways for } \\
\text { sighted virtual driver agents }\end{array}$} \\
\hline Type of driver & $48.3 \mathrm{~km} / \mathrm{h}$ & $66.0 \mathrm{~km} / \mathrm{h}$ & $80.5 \mathrm{~km} / \mathrm{h}$ & $48.3 \mathrm{~km} / \mathrm{h}$ & $66.0 \mathrm{~km} / \mathrm{h}$ & $80.5 \mathrm{~km} / \mathrm{h}$ \\
\hline $\begin{array}{l}\text { Inexperienced } \\
\text { Low }\end{array}$ & $23.3(1.739)$ & $34.0(1.860)$ & $46.8(2.142)$ & $26.6(1.985)$ & $36.9(2.015)$ & $46.2(2.063)$ \\
experience & $23.9(1.780)$ & $32.8(1.822)$ & $44.7(2.038)$ & $27.3(2.041)$ & $40.0(2.184)$ & $44.1(1.969)$ \\
Experienced & $24.1(1.792)$ & $32.6(1.749)$ & $41.3(1.867)$ & $27.5(2.054)$ & $35.5(1.938)$ & $40.7(1.817)$ \\
\hline
\end{tabular}

(b)

Table 6.3 Comparing mean driving headways during low $P$ car following condition, [Colbourn et al 1978], (a) unsighted (b) sighted virtual driver agents 
Table $6.3 \mathrm{a}$ and $6.3 \mathrm{~b}$ used the same data (for speeds $48.3 \mathrm{~km} / \mathrm{h}, 66.0 \mathrm{~km} / \mathrm{h}$ and 80.5 $\mathrm{km} / \mathrm{h}$ ) from Colbourn's study to assess unsighted and sighted estimation of distance and time headways. Table 6.3 shows a general overestimation by both sighted and unsighted driver agents of Colbourn's distance and time headways calculation for all speeds, except in the $80.5 \mathrm{~km} / \mathrm{h}$ speed range. One possible reason for the overestimation is explained by the fact that at lower speeds, the preferred following distance of the driver agents is calculated to be higher than their initial headway. This caused drivers to try to adjust to their preferred front distance, leading to slowing down and increasing their time headway. However, in the higher speeds drivers have a 'comfortable' headway compared to their initial headway. Sighted driver agents performed slightly better than unsighted drivers.

\subsubsection{Discussion of Comparative Studies}

The mathematical theory of driver behaviour formulated by Lee (Lee 1976) has formed a central part in the evaluation of SD-SIM's perceptual factors. In many driving behaviour studies, subjective interpretation of driver behaviour is often enough to understand the factors and characteristics of the behaviour. However, the ability to quantify these factors provides additional means of understanding.

The simple evaluation of SD-SIM's performance based on previously published data has shown that SD-SIM perceptual capability compares favourably. Driver agents in SD-SM are capable of processing optical flow information through the functionality of SEE.

However, it should be noted that the evaluation method based on previously published data is prone to errors due mainly to the inherent assumptions made in defining scenarios in SD-SIM. Data reported in the literature are based on different assumptions, different experimental set ups, etc. Transforming these data to perform calibration equally requires assumptions to be made. Although care has been taken to establish parity in assumption and calibration, a further and more robust evaluation is required. This is discussed is section 6.3 . 


\subsection{Comparison with Real World Scenarios}

In addition to the evaluation of SD-SIM based on data obtained from the literature, this section goes further in conducting driving experiments to generate accurate measurements in known circumstances to assess the quantitative performance of the SEE and IVD modules within SD-SIM. Furthermore, although the use Police drivers and subjects provided an opportunity to obtain data on their performance, this was a secondary objective and as such these experiments were not intended to be statistically significant.

\subsubsection{Data Collection}

Distance and speed data have been collected using Home Office equipment together with subjects' estimates, to calibrate and validate SEE and IVD. The data collected from the equipment reading and subjects will be compared with those produced by the SEE and IVD modules.

\subsubsection{General Method}

Three separate types of experiments were conducted over a two-day period. In the first day, measurements of distances and speeds while driving on the M1 motorway (experiment 1 - motorway experiment) were collected using Home Office equipment. This experiment did not include measurement of human subjects. In the same day, estimates of distances and speeds using the same equipment were made, together with human subjects' estimates in (experiment 2 - 'stop and go' experiment) conducted at Donnington Park Race Track in Leicestershire.

Tasks carried out on the second day at Donnington Park Race Track centred on dynamic speed estimation (experiment 3 - dynamic situation experiment). This involved using in-car detectors, located in a vehicle travelling at a fixed speed, to measure the speed of a vehicle travelling at different speeds. Several human subjects were also included in this experiment. 


\section{Equipment}

Two Police vehicles were used, each equipped with forward facing camera and video recording system to monitor and record the scene. The camera-video equipment was fitted in the vehicle using an 'internal universal vehicle interface' system. Recorded information included time data, speed, and distance travelled by the vehicle are stamped on the video. A hand held radar rangefinder was mounted for forward looking since this was not part of the instrumentation of the vehicles. The radar gun can be adjusted to measure and display distance and speed to the lead vehicle. The radar rangefinder has an operational speed range of 5 to $299 \mathrm{mph}$ with a speed display accuracy of $\pm 1 \mathrm{mph}$ and target range of 1.52 meters to 1,372 meters with a range display accuracy of $\pm 0.3 \mathrm{~m}$. Finally, a two-way radio for communicating with other drivers was also available and used to give instructions to the lead vehicle driver.

\section{Subjects}

A total of 17 subjects, 13 males and 4 females, took part in the two-day experiments. The first day involved 10 subjects with 7 subjects taking part on the second day. The mean age and driving experience of the subjects were 24.8 years and 6.4 years respectively. All the subjects were holders of a current 'full' British driving license.

\section{Procedure}

In experiment 1 (motorway experiment), two vehicles, trail vehicle and lead vehicle drove on a British motorway (M1-North), between junction 20 and junction 24 . The equipment in the two vehicles were synchronised so that the vehicle's video outputs have a single base line measurement of distance and speed. The motorway experiment was intended to collect relative distance and speed measurements between the vehicles. The Police driver of the trail vehicle was instructed to follow the lead vehicle for a period of time and to change lane to pass the lead vehicle when prompted. This allows vehicle trajectory data on lane changing behaviour to be collected using the onboard vehicle equipment.

The video images from the two cameras were processed with image processing software to extract useful image frames at 5 seconds interval. The relative distances and speeds between the vehicles were obtained by subtracting the absolute distance 
and speed of two consecutive frames of the trail vehicle and the lead vehicle. This process is illustrated in figure 6.2.

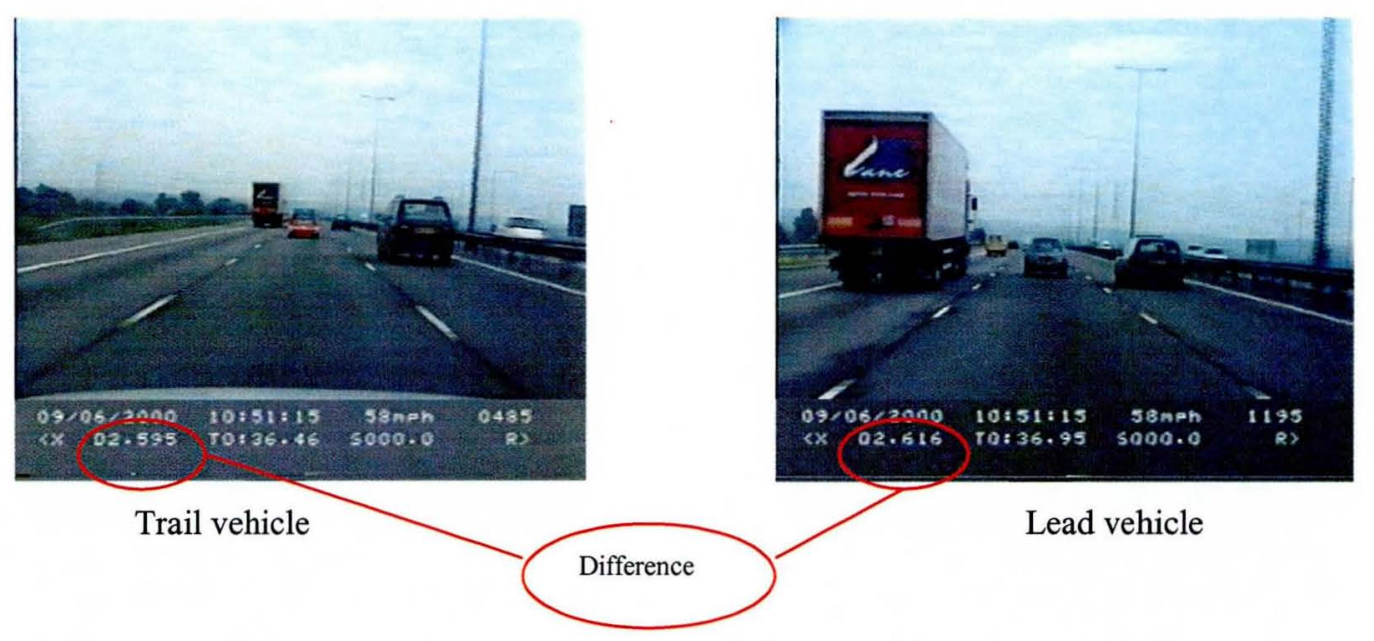

Figure 6.2 Extracting relative distances from synchronised video images

In experiment 2 ('stop and go' experiment), an approaching target vehicle was stationary at measured distances but travelled at various speeds over each time interval. Figure 6.3 shows five images of the approaching vehicle as viewed from the stationary vehicle. The distances to the vehicle positions were obtained from the equipment. At the same time, each subject and an experimenter sat in a stationary vehicle. The subject sat in the front passenger seat and also wore a pair of goggles to record the scene from the subject's eye level.

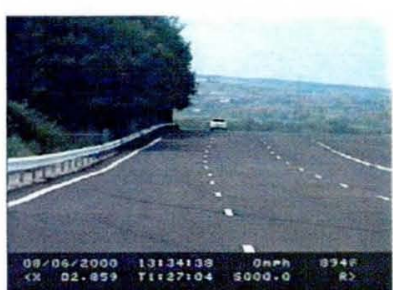

Frame 1, Time 0 seconds

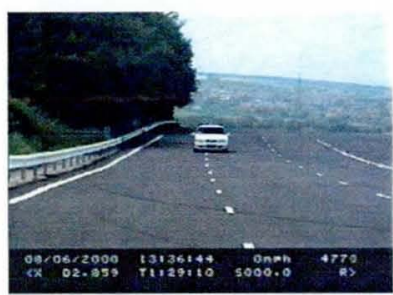

Frame 4, Time 15 seconds

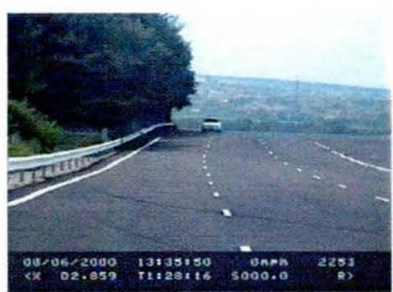

Frame 2, Time 5 seconds

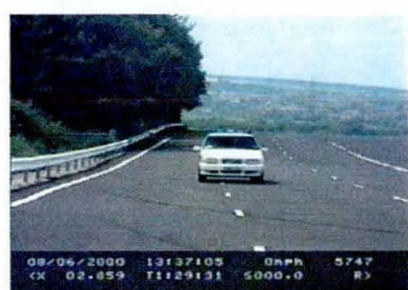

Frame 5, Time 20 seconds

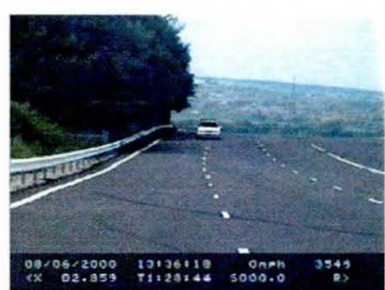

Frame 3, Time 10 seconds

Figure 6.3 Scene captured by police cameras as viewed from stationary vehicle 
The procedural sequence for data collection in experiment 2 involved:

1. Each subject was handed a clipboard with data recording sheets. Instructions of the experiments and their implication to safe driving were explained to the subject.

2. A test run was performed to familiarise the subject with the general procedures. Each subject was allowed to make some estimate of distance and speed. These estimates were compared to actual readings obtained from the equipments to calibrate the instruments and establish baseline measurements but these readings were not used in the final analysis.

3. The target vehicle moved from rest for a period of 5 seconds then stopped.

4. The experimenter prompted the subject at every 5 seconds to indicate on their recording sheet their perception of the experimental tasks. The procedure for all the experimental tasks was identical for all subjects and included:-

a) To indicate by marking on the scale and adding a value (given in any units) of what their estimate of the position (distance) to the target vehicle at this time period was.

b) To indicate by marking on the scale and adding a value (given in any units) of what their estimate of the speed of the target vehicle at this time period was.

5. The procedure for each subject was repeated twice. The duration of each run of the experiment was 20 seconds (affected by the length of the section of the race track used). The tools for the data collection process are given in Appendix C.3.

6. The actual positions of the target vehicle were recorded using the radar gun equipment. Images of the approaching target vehicle were captured using the head mounted camera and the forward facing police camera. 
Experiment 3 (dynamic situation) was conducted in day two and similarly to experiment 2, with the intention of collecting data from the equipment in a dynamic situation. However, the dynamic situation experiment also involved 7 subjects, sitting in the trail vehicle as passengers whilst following a target lead vehicle. Both vehicles were driven by Police officers. Nominal target vehicle speeds of 20, 40 and $60 \mathrm{mph}$ were used. The subjects did not know the speed at which they were travelling. The procedural sequence for data collection in this experiment involved:

1. Each subject was handed a clipboard with data recording sheets. Instructions of the experiments and their implication to safe driving were explained to the subject.

2. The experimenter prompted the subject at every 5 seconds to indicate on their recording sheet by marking on the scale and adding a value (given in any units) of what their estimate of the speed of the lead vehicle at this time period was.

3. The procedure for each subject was repeated twice. The duration of each run of the experiment was 20 seconds (affected by the length of the section of the race track used). The tools for the data collection process are given in Appendix C.3.

4. As in the previous experiments, the actual speed values were recorded using the radar gun equipment.

In the collection of data for these experiments, it should be noted that the use of the scales was intended to remove possible errors that individuals may make in converting their perception to numerical values at the time of perception. This relates to problems usually associated with verbalisation techniques. Leibowitz et al (1993) provides a good discussion on the accuracy of verbal and nonverbal methods. A more formal description of the tools used in these experiments is given in Appendix C. 


\subsubsection{Data Sets}

The data recorded from the instruments and subjects' estimates in the experiments constituted the data sets for the validation of SEE and IVD modules. The instrumented vehicle data included processed video images from experiment 1 , to obtain vehicle accelerations and trajectories in terms of relative distances and speeds between the vehicles. In addition, the equipment data also included radar gun readings of distances and speeds from experiments 2 and 3. The data from the subjects mainly included estimates of distances and speeds recorded alongside the scales.

Table 6.4 shows the relative distances and speeds obtained from the video recordings in experiment 1 . The video data was processed for 70 seconds. Table 6.5 shows Mean Actual Distance (MAD) obtained from the laser gun reading, compared to subject estimates of Mean Estimated Distance (MED) in the 'stop and go' experiment (experiment 2). The data was recorded for the 10 subjects.

\begin{tabular}{|c|c|c|}
\hline Time $[\mathrm{s}]$ & Relative distance $[\mathrm{m}]$ & Relative speed $[\mathrm{m} / \mathrm{s}]$ \\
\hline 5 & 33.796 & 0.000 \\
\hline 10 & 38.624 & 0.447 \\
\hline 15 & 32.187 & -0.447 \\
\hline 20 & 32.187 & 0.447 \\
\hline 25 & 28.968 & 0.000 \\
\hline 30 & 32.187 & -0.894 \\
\hline 35 & 27.359 & 0.447 \\
\hline 40 & 41.843 & 0.894 \\
\hline 45 & 41.843 & 1.341 \\
\hline 50 & 53.108 & 2.235 \\
\hline 55 & 62.764 & 1.788 \\
\hline 60 & 67.592 & 1.341 \\
\hline 65 & 74.030 & 0.000 \\
\hline 70 & 67.592 & 0.000 \\
\hline
\end{tabular}




\begin{tabular}{|c|c|c|c|c|c|c|c|c|c|c|c|}
\cline { 2 - 11 } \multicolumn{2}{|c|}{} & Subj1 & Sub2 & Subj3 & Subj4 & Subj5 & Subj6 & Subj7 & Subj8 & Subj9 & Subj10 \\
\hline $\begin{array}{c}\text { Time } \\
{[\mathrm{s}]}\end{array}$ & $\begin{array}{c}\text { MAD* } \\
{[\mathrm{m}]}\end{array}$ & $\begin{array}{c}\text { MED* } \\
{[\mathrm{m}]}\end{array}$ & $\begin{array}{c}\text { MED } \\
{[\mathrm{m}]}\end{array}$ & $\begin{array}{c}\text { MED } \\
{[\mathrm{m}]}\end{array}$ & $\begin{array}{c}\text { MED } \\
{[\mathrm{m}]}\end{array}$ & $\begin{array}{c}\text { MED } \\
{[\mathrm{m}]}\end{array}$ & $\begin{array}{c}\text { MED } \\
{[\mathrm{m}]}\end{array}$ & $\begin{array}{c}\text { MED } \\
{[\mathrm{m}]}\end{array}$ & $\begin{array}{c}\text { MED } \\
{[\mathrm{m}]}\end{array}$ & $\begin{array}{c}\text { MED } \\
{[\mathrm{m}]}\end{array}$ & $\begin{array}{c}\text { MED } \\
{[\mathrm{m}]}\end{array}$ \\
\hline 0.00 & 175.53 & 200.00 & 150.00 & 274.20 & 157.00 & 300.00 & 150.00 & 250.00 & 191.94 & 228.50 & 200.00 \\
\hline 5.00 & 153.25 & 170.00 & 100.00 & 228.50 & 155.00 & 250.00 & 130.00 & 200.00 & 182.80 & 182.80 & 190.00 \\
\hline 10.00 & 122.16 & 150.00 & 100.00 & 137.10 & 130.00 & 220.00 & 100.00 & 180.00 & 109.68 & 137.10 & 170.00 \\
\hline 15.00 & 88.88 & 110.00 & 50.00 & 73.12 & 100.00 & 200.00 & 80.00 & 100.00 & 91.40 & 82.26 & 100.00 \\
\hline 20.00 & 54.40 & 25.00 & 20.00 & 45.70 & 25.00 & 150.00 & 60.00 & 50.00 & 45.70 & 18.28 & 40.00 \\
\hline
\end{tabular}

Note: $\mathbf{M A D}^{*}-$ Mean Actual Distance

MED $^{*}$ - Mean Estimated Distance

Subj. - Subject

Table 6.5 Actual measured distance compared to subjects estimates (experiment 2)

\subsubsection{Validation of Scene En-capturing Evaluation (SEE)}

The validation of SEE is based on the quantitative assessment of SEE's performance in terms of virtual driver estimates of distance and speed compared with measured data along with subjects estimates of distance and speed. It should be emphasised here that the main purpose of performing the experiments was to generate quantitative test data from real-life instruments to assess the current performance of the SEE against these equipment. The benefit of using human subjects in the experiments was that an initial crude comparison could be made between them and SEE, allowing the inherent fuzziness in human perception to be considered within future development of the SEE's parameters.

\subsubsection{Calibration of Scene En-capturing Evaluation (SEE)}

In calibrating SEE, the following vision model parameters were used (refer to chapter 4 for more detail description of these parameters):

Eye dimensions: $n=50$ rows and $m=50$ columns in both left and right eye matrices.

Eye separation: $0.06 \mathrm{~m}$.

Horizontal field of view: 120 degrees.

Vertical field of view: 50 degrees upwards and 100 degrees downwards from horizontal 
To calibrate the accuracy of SEE in terms of measured data and subjects estimates, two methods have been employed to measure the goodness of fit of the simulated results:

$$
M P E=\frac{1}{T} \sum_{t=1}^{t=T} P_{t} \quad \text { RMS Percent Error }=\sqrt{\frac{1}{T} \sum_{i=1}^{i=T}\left(P_{t}^{2}\right)}
$$

Where,

$$
\begin{aligned}
M P E= & \text { mean percent error } \\
T= & \text { product of the number of time steps and the number of driver } \\
& \text { subjects in each experiment. }
\end{aligned}
$$

The percent error, $P_{t}$, corresponds to the difference in mean estimated value and mean actual value and is given by

$$
P_{t}=\left(\frac{e_{t}-a_{t}}{a_{t}}\right) \times 100
$$

Where,

$$
\begin{aligned}
& e_{t}=\text { mean value estimated by a driver subject or the vision model at a } \\
& \quad \text { particular time. } \\
& a_{t} \quad \text { mean actual value at the same time. }
\end{aligned}
$$

\subsubsection{Distance Estimation}

The validity of distance estimation by SEE is based on comparing virtual drivers' estimates with measured data and subjects estimates obtained from experiments 1 and 2. The instantaneous geometric distance $D$ perceived by the driver agent is given by

$$
\begin{aligned}
& D=\sqrt{\left.\left(n_{1} t\right)^{2}+\left(n_{2} t\right)^{2}+\left(n_{3} t\right)^{2}\right)} \\
& =|t| \sqrt{n_{1}{ }^{2}+n_{2}{ }^{2}+n_{3}{ }^{2}}
\end{aligned}
$$


Where,

$n_{1}, n_{2}, n_{3}=$ are components of the outward normal vector for an object face.

$t=$ is the intersection parameter of each ray on the object face.

The distance data obtained from the equipment in the motorway and 'stop and go' experiments were used to configure the parameters of SEE within SD-SIM. The simulation to replicate the observed distance measured in the motorway experiment (experiment 1) was run for 70 seconds. The errors in the distance estimates between measured data and SEE are shown in figure 6.4. Although the agent's estimates are close to the measured value, there is underestimation and overestimation of distance. The comparison of SEE's results with the measured data demonstrates the mathematical accuracy of SEE's algorithms.

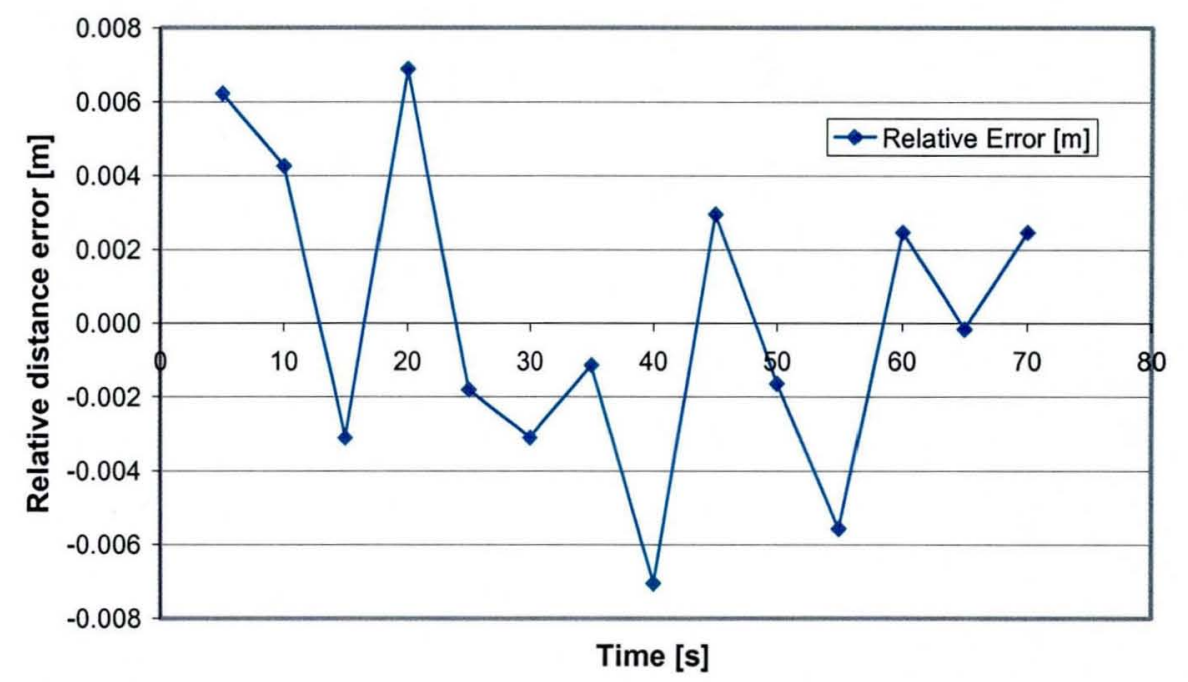

Figure 6.4 Distance estimation errors by virtual driver agents

In the 'stop and go' experiment (experiment 2), the dimensions of the target vehicle and its measured discrete position was assembled into a vehicle object and presented to an observing virtual driver agent with SEE capability. The simulation was run for 20 seconds. Table 6.6 and figure 6.5 compare measured data (i.e. mean actual distance of the laser gun readings), and mean estimates of distance made by the driver subjects with virtual driver agents estimates using SEE's capability. At time 0 seconds the virtual driver agents slightly underestimate the mean actual distance of $175.53 \mathrm{~m}$ by $0.99 \%$. In comparison, the subjects' overestimation is relatively high at $19.73 \%$. At 20 
seconds virtual driver agents and subjects underestimate the mean actual distance of $54.40 \mathrm{~m}$ by $-3.11 \%$ and $-11.82 \%$, respectively. It is clear from figure 6.5 and the measures in table 6.6 that the vision model consistently provides a better comparison with measured data than that obtained from the subjects. Further discussion of these results is given in the discussion of results section.

\begin{tabular}{cccc}
\hline \multicolumn{4}{c}{ Distance estimation } \\
\hline $\begin{array}{c}\text { Time [s] } \\
{[\mathrm{T}]}\end{array}$ & $\begin{array}{c}\text { Mean Actual } \\
\text { Distance [m] } \\
{[\mathrm{MAD}]}\end{array}$ & $\begin{array}{c}\text { Mean Estimated } \\
\text { Distance [m] } \\
\text { [Driver subjects] }\end{array}$ & $\begin{array}{c}\text { Eye resolution: } 50 \text { by } 50 \\
\text { Mean Estimated } \\
\text { Distance [m] } \\
\text { [Vision model] }\end{array}$ \\
\hline 0 & 175.53 & 210.16 & 173.79 \\
5 & 153.25 & 178.91 & 151.52 \\
10 & 122.16 & 143.39 & 120.44 \\
15 & 88.88 & 98.68 & 87.18 \\
20 & 54.40 & 47.97 & 52.71 \\
Statistical measure & & 9.49 & -1.76 \\
Mean percent error (\%) & 36.80 & 2.00 \\
RMS percent error (\%) & &
\end{tabular}

Table 6.6 Comparison of distance estimation in experiment 2

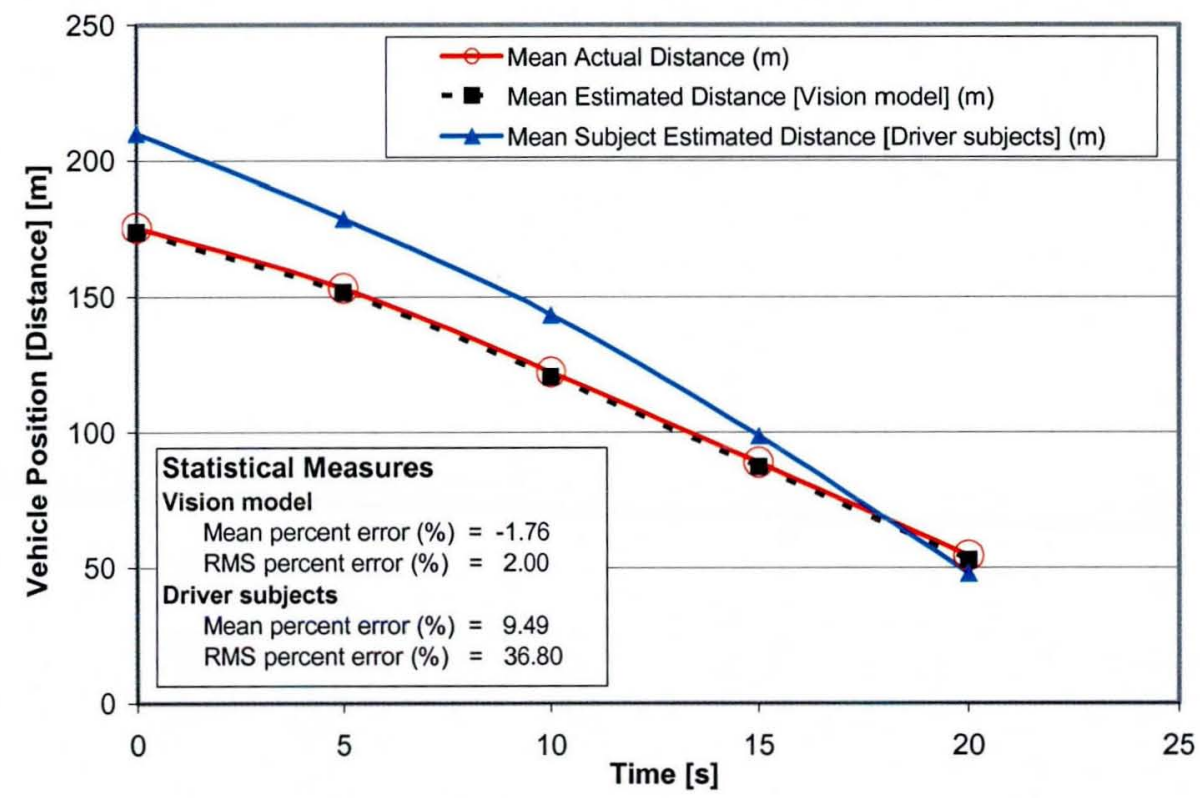

Figure 6.5 Estimation of distance from discrete position measurements of target vehicle in experiment 2 


\subsubsection{Speed Estimation}

The validity of speed estimation by SEE is based on comparing virtual drivers' estimates with measured speed data and subjects estimates obtained from experiments 1,2 and 3 . In experiment 2, the average speed between vehicle positions, perceived by the vision model due to a change in image patterns between consecutive time steps can be simply obtained using the relation in equation 6.16 .

$$
S\left(t^{\prime}\right)=\frac{\Delta x}{\Delta t}
$$

Where:

$$
\begin{aligned}
\Delta x= & \text { estimate of relative or apparent distance change due to change in } \\
& \text { image patterns between consecutive frames (meters). Same as the } \\
& \text { difference between } D \text { for the object in each frame. } \\
\Delta t \quad= & \text { time step between two consecutive frames (seconds). } \\
S\left(t^{\prime}\right)= & \text { average speed between vehicle positions (meters per seconds) where } \\
& \mathrm{t}^{\prime} \text { is the mid time step time. }
\end{aligned}
$$

The same configuration of SD-SIM for distance estimation was performed for the comparison of speed estimation by virtual driver agents with measured speed data in experiment 1 . The errors in the speed estimation by virtual driver agents for this experiment are shown in figure 6.6. As with the distance estimation, the virtual driver agent's estimates are close to the measured value. The graph also shows under-and overestimation of speed.

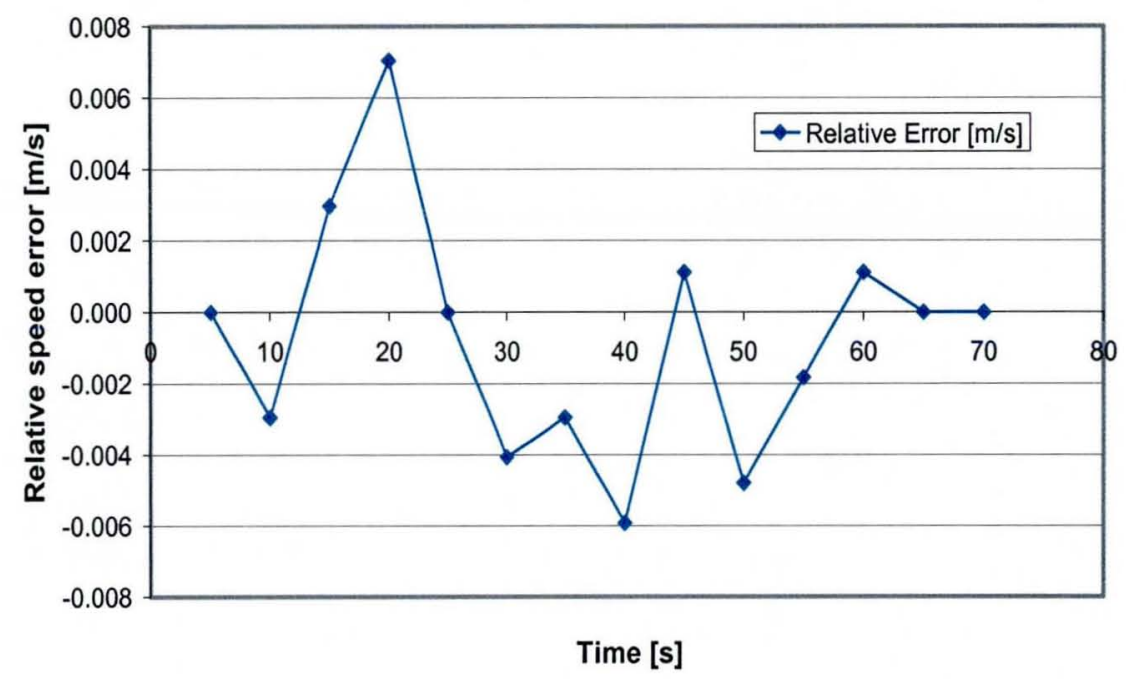

Figure 6.6 Speed estimation errors by virtual driver agents 
The estimation of speed by virtual driver agents in experiment 2 was compared with measured data and subjects estimates of speed. It should be noted that the speeds in this experiment are calculated based on averages due to the stop and go nature of the experiment, rather than the relative speeds. Table 6.7 and figure 6.7 compare average speeds, estimated by the vision model, calculated from measured data and derived from driver subjects' estimates. The results obtained show a good agreement between the estimates made by SEE and actual mean speed value but human subject estimates are poor in comparison.

\begin{tabular}{cccc}
\hline \multicolumn{4}{c}{ Speed estimation } \\
\hline $\begin{array}{c}\text { Time }[\mathrm{s}] \\
{[\mathrm{T}]}\end{array}$ & $\begin{array}{c}\text { Mean Actual } \\
\text { Speed [m/s] } \\
{[\mathrm{MAS}]}\end{array}$ & $\begin{array}{c}\text { Mean Estimated } \\
\text { Speed [m/s] } \\
\text { [Driver subjects] }\end{array}$ & $\begin{array}{c}\text { Eye resolution: } 50 \text { by } 50 \\
\text { Mean Estimated } \\
\text { Speed [m/s] } \\
\text { [Vision model] }\end{array}$ \\
\hline 2.5 & 4.46 & 6.25 & 4.45 \\
7.5 & 6.22 & 7.10 & 6.22 \\
12.5 & 6.66 & 8.94 & 6.65 \\
17.5 & 6.90 & 10.14 & 6.89 \\
Statistical measure & & & \\
Mean percent error (\%) & 32.85 & -0.03 \\
RMS percent error (\%) & 87.43 & 0.04 \\
\hline \multicolumn{2}{l}{}
\end{tabular}

Table 6.7 Comparison of speed estimation in experiment 2

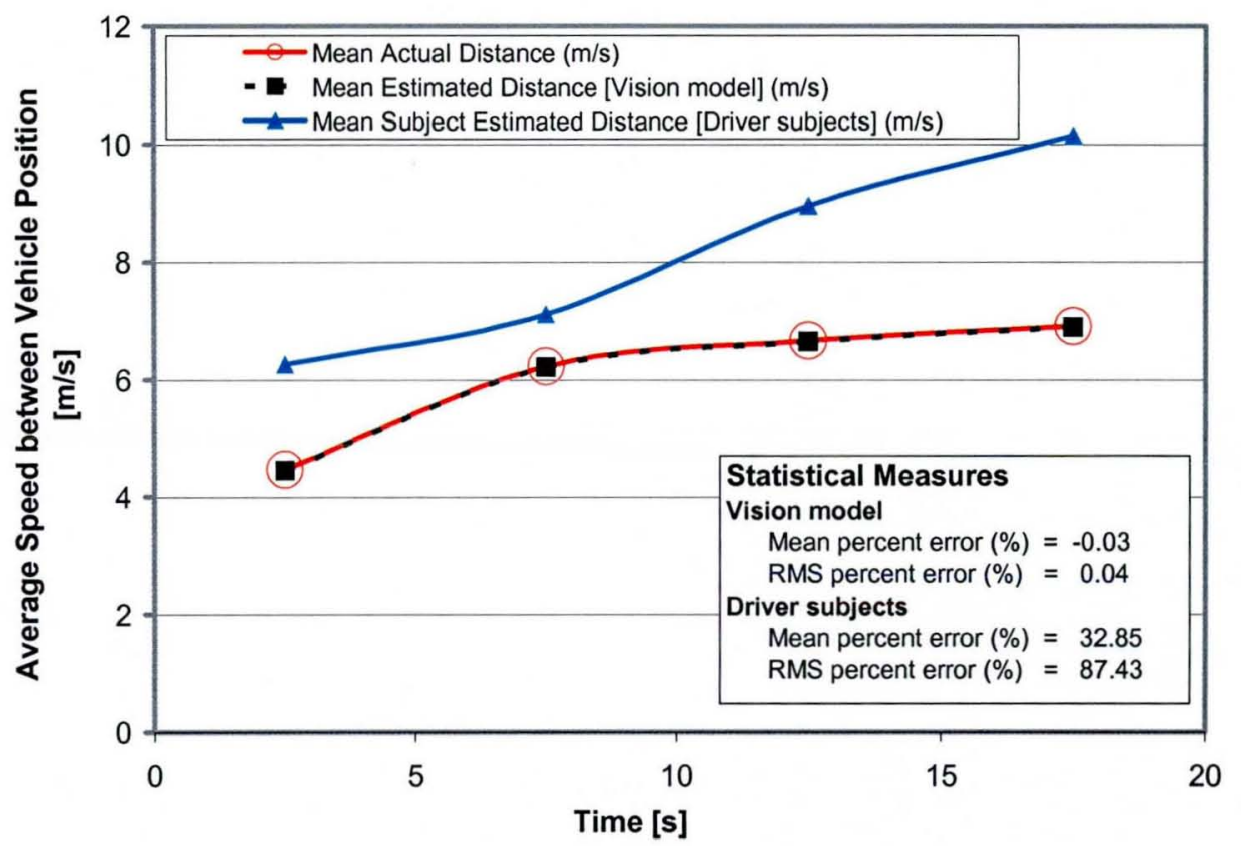

Figure 6.7 Estimation of speed from discrete position measurements of target vehicle in experiment 2 
In experiment 3, the dynamic speed estimation was replicated by configuring SD-SIM with two vehicles. Nominal target vehicle speeds in the simulation was set at 20, 40 and $60 \mathrm{mph}$. Table 6.8 and figure 6.8 compare mean measured speeds of the target vehicle with mean values estimated by the vision model and the subject drivers. As in the previous experiment, there is a general trend for the vision model to underestimate speeds, whilst the subject drivers tended to overestimate. The mean percent error for the vision model reduced from $-8.18 \%$ at $20 \mathrm{mph},-4.15 \%$ at $40 \mathrm{mph}$ and $-2.83 \%$ at $60 \mathrm{mph}$. This is in respect to the RMS values of $8.19 \%$ at $20 \mathrm{mph}, 4.15$ at $40 \mathrm{mph}$ and $2.84 \%$ at $60 \mathrm{mph}$. While driver subjects' overestimation at $20 \mathrm{mph}$ is relatively higher at $14.19 \%$, there was an improved estimation of speed at $40 \mathrm{mph}$ with a percent error of $4.64 \%$. Further discussion of the results is given in the discussion of results section.

\begin{tabular}{|c|c|c|c|c|c|c|c|c|c|}
\hline \multicolumn{10}{|c|}{ Speed estimation } \\
\hline $\begin{array}{c}\text { Time } \\
{[\mathrm{T}]}\end{array}$ & \multicolumn{3}{|c|}{$\begin{array}{c}\text { Mean Actual } \\
\text { Speed [mph] } \\
\text { [MAS] }\end{array}$} & \multicolumn{3}{|c|}{$\begin{array}{l}\text { Mean Estimated } \\
\text { Speed [mph] } \\
\text { [Driver subjects] }\end{array}$} & \multicolumn{3}{|c|}{$\begin{array}{c}\text { Eye resolution: } 50 \text { by } 50 \\
\text { Mean Estimated } \\
\text { Speed [mph] } \\
\text { [Vision model] }\end{array}$} \\
\hline$[\mathrm{s}]$ & @ 20 & @ 40 & @ 60 & @ 20 & @ 40 & @ 60 & @ 20 & @ 40 & $@ 60$ \\
\hline 0 & 20.75 & 40.75 & 59.38 & 21.25 & 41.25 & 63.13 & 19.07 & 39.07 & 57.68 \\
\hline 5 & 20.25 & 40.75 & 60.00 & 23.38 & 43.13 & 66.88 & 18.57 & 39.07 & 58.31 \\
\hline 10 & 21.13 & 40.50 & & 24.63 & 43.13 & & 19.45 & 38.82 & \\
\hline 15 & 20.25 & & & 24.63 & & & 18.57 & & \\
\hline 20 & 20.25 & & & 26.13 & & & 18.57 & & \\
\hline \multicolumn{10}{|c|}{ Statistical measure } \\
\hline \multicolumn{4}{|c|}{ Mean percent error $(\%)$} & 14.19 & 4.64 & 8.99 & -8.18 & -4.15 & -2.83 \\
\hline \multicolumn{4}{|c|}{ RMS percent error (\%) } & 29.67 & 20.68 & 17.71 & 8.18 & 4.15 & 2.84 \\
\hline
\end{tabular}

Table 6.8 Comparison of speed estimation in experiment 3 


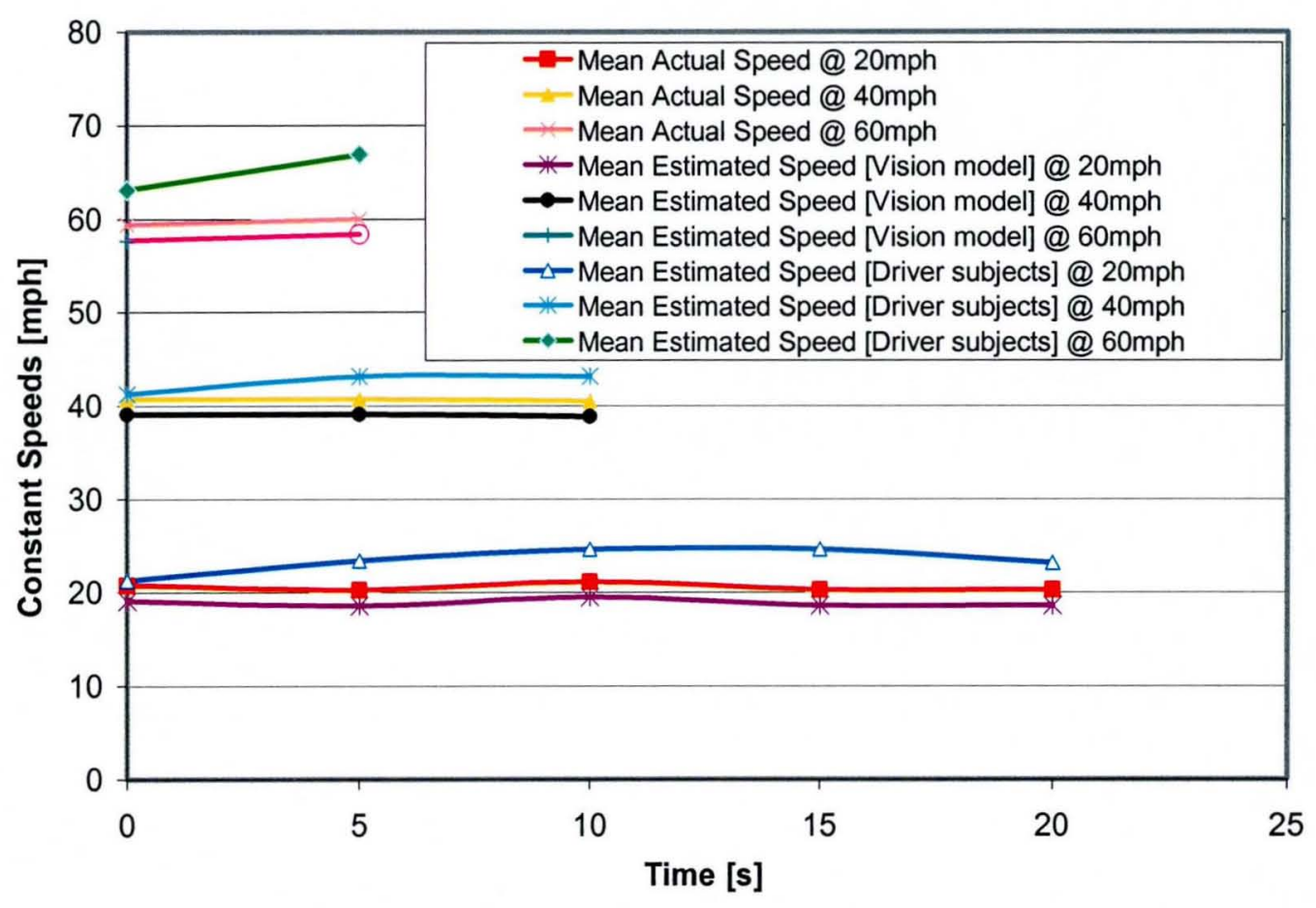

Figure 6.8 Estimation of target vehicle speed in experiment 3

\subsubsection{Eye Resolution and Computational Considerations}

The computation of SD-SIM is affected by many factors but one of the main factors is the computation performed by the vision model. At the same time, the ability for SEE to discriminate spatial properties depends on the eye resolution and frame rate. The problem of balancing eye resolution and computational efficiency is an optimisation problem. The implications of varying eye resolution and frame rate for the distance and speed estimation discussed above are discussed here. An optimum solution for configuring SD-SIM is also suggested. The following sections discuss the computational considerations in running SD-SIM.

\section{Influence of Eye Resolution on Computational Efficiency}

SD-SIM provides facilities to display images using the Log Images (L.I.) or Not to Log Images (N.L.G.). When the checkbox is not activated, SEE runs in the background performing all the necessary vision calculations for each driver agent. Table 6.9 illustrates typical mean times taken to perform the distance and speed calculations discussed in section 6.3.2.2 and 6.3.2.3. The simulation was run a Pentium II, 128MB RAM for duration of 20 seconds. Each simulation execution was 
timed before and after the simulation run. Considering combined human reaction and clock errors of \pm 3 seconds, it is obvious from the table that in general computational efficiency in SD-SIM decreases when the vision facilities are active. This is also the trend when different eye resolutions are defined for SEE i.e. the higher the eye resolution matrix, the slower the computation.

The computational overhead in SEE is an inherent problem of ray tracing techniques since calculations have to be carried out for each ray projected into the scene. However, there are also other factors that affect computational efficiency such as time steps e.g. shorter time step with longer end time imposes increasing computational expense on the system. At the same time, being able to identify sections of the simulation where decisions were made is dependent on defining appropriate time steps. The problem of computational efficiency currently limits the number of driver agents that can be simulated.

\begin{tabular}{|c|c|c|c|c|c|c|c|c|c|c|}
\hline \multirow{3}{*}{$\begin{array}{c}\text { SD- } \\
\text { SIM } \\
\text { without } \\
\text { SEE }\end{array}$} & \multicolumn{10}{|c|}{ SD-SIM with SEE Active and Different Eye Resolutions } \\
\hline & \multicolumn{2}{|c|}{$\begin{array}{c}\text { col } \times \text { row } \\
20 \times 25\end{array}$} & \multicolumn{2}{|c|}{$\begin{array}{c}\text { col } x \text { row } \\
50 \times 50\end{array}$} & \multicolumn{2}{|c|}{$\begin{array}{c}\text { col } x \text { row } \\
100 \times 50\end{array}$} & \multicolumn{2}{|c|}{$\begin{array}{l}\text { col } \times \text { row } \\
100 \times 100\end{array}$} & \multicolumn{2}{|c|}{$\begin{array}{l}\text { col } \times \text { row } \\
300 \times 100\end{array}$} \\
\hline & L.I & N.L.G & L.I & N.L.G & L.I & N.L.G & L.I & N.L.G & L.I & N.L.G \\
\hline $0.03 \mathrm{~s}$ & $0.10 \mathrm{~s}$ & $0.09 \mathrm{~s}$ & $5.04 \mathrm{~s}$ & $4.31 \mathrm{~s}$ & $9.09 \mathrm{~s}$ & $9.04 \mathrm{~s}$ & $19.73 \mathrm{~s}$ & $18.09 \mathrm{~s}$ & $57.67 \mathrm{~s}$ & $54.08 \mathrm{~s}$ \\
\hline
\end{tabular}

Table 6.9 Computational efficiency for different eye resolution

\section{Optimisation of Eye Resolutions}

Since the most computationally costly component of SD-SIM is dependent on eye resolutions, it is important to assess how this process can be optimised. The current process of defining different eye resolutions is ad hoc. Therefore, to optimise this process, the requirement to determine the number of rays required for detecting an object at a maximum distance $\mathrm{S}_{\mathrm{x}}$, with a vehicle width $\mathrm{w}$ is illustrated in figure 6.9. For the purpose of deriving the optimisation relation, this figure assumes three rays subtending a small angle $\theta$, with all rays hitting the vehicle in front. Considering only the horizontal field of view, (ignoring the vertical field of view since driver vision is restricted mainly due to the front windscreen), the relationship between the visual angle $\theta, w$ and $S_{x}$ is given by the equation 6.17 . 


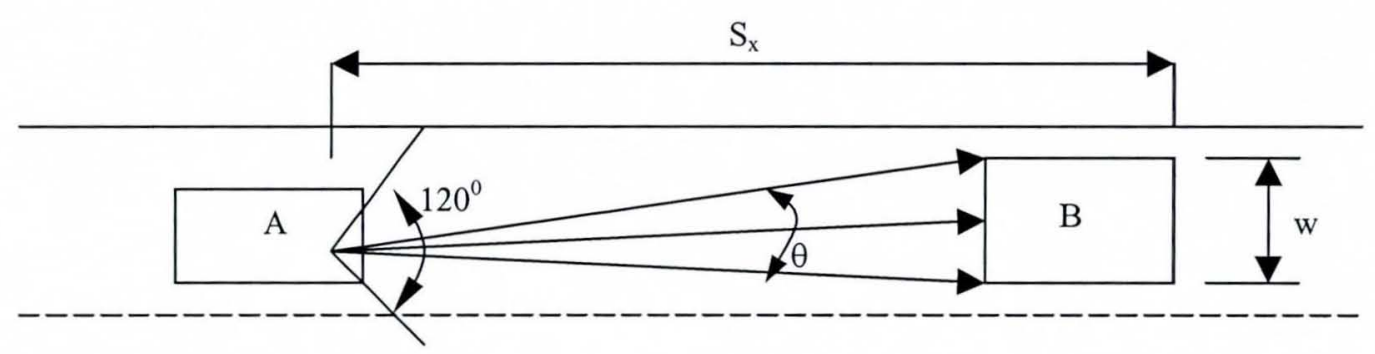

Figure 6.9 Determine the eye resolution required to detect an object at maximum distance

By design, the maximum separation distance, $S_{x}$ between vehicle $A$ and $B$, is the largest separation value searching through all the simulation time steps and $\mathrm{w}$ is obtained from the vehicle dimensions. Equation 6.18 is derived to describe the smallest inter-ray spacing that determines the maximum number of rays, $\mathrm{N}_{\max }$ required to detect vehicle, $B$.

$$
\begin{aligned}
& \theta=\frac{w}{\mathrm{~S}_{\mathrm{x}}} \quad \theta \rightarrow 0 \\
& \mathrm{~N}_{\max }=\frac{\left(120 \times \frac{2 \pi}{360}\right)}{\frac{\theta}{2}}
\end{aligned}
$$

Therefore, the maximum number of columns within an eye required to detect vehicle $B$ at the separation distance $S_{x}$ is given as:

$$
N_{\max }=\frac{4 \pi}{3 \theta}
$$

To determine the maximum number of columns within an eye required to detect an object, SD-SIM is first run without the vision model, to calculate $\mathrm{S}_{\mathrm{x}}$. $\theta$ is then calculated using the value of $\mathrm{S}_{\mathrm{x}}$. From this $N_{\max }$ is calculated and SD-SIM is again run with the optimised eye resolution defined. 


\section{Influence of Eye Resolution on Estimation and Perception Errors}

Using the optimisation approach, four example eye resolutions have been generated for the distance and speed estimations in sections 6.3.2.2 and 6.3.2.3. Tables 6.10 and 6.11 present the results for higher eye resolutions. Figures 6.10 and 6.11 present the errors as a function of eye resolutions in the estimation of distance and speeds. The tables and figures show clear improvements in distance and speed estimations, i.e. smaller mean errors using higher eye resolutions. However comparing these figures with table 6.9, it is obvious this improvement has been achieved at higher computational cost. Therefore, the choice of eye resolution is compromised with the need to manage resources and achieve computational efficiency. Some of these factors have led to the choice of an eye resolution of 50 by 50 as an initial starting point in the configuration of SD-SIM.

\begin{tabular}{|cccccccc|}
\hline \multicolumn{7}{|c|}{ Mean estimated distance [SEE], with different eye resolution } \\
\hline $\begin{array}{l}\text { Time } \\
\text { step (s) } \\
{[\mathrm{T}]}\end{array}$ & $\begin{array}{l}\text { Mean Actual } \\
\text { Distance }(\mathrm{m})\end{array}$ & $\begin{array}{l}\text { Mean Estimated } \\
\text { Distance }(\mathrm{m})\end{array}$ & $\begin{array}{l}\text { col.row } \\
\text { [Driver subject] }\end{array}$ & $\begin{array}{l}50 \times 25 \\
\text { col.row }\end{array}$ & $\begin{array}{l}\text { col.row } \\
100 \times 50\end{array}$ & $\begin{array}{l}\text { col.row } \\
100 \times 100\end{array}$ & $\begin{array}{l}\text { col.row } \\
\text { 300x100 }\end{array}$ \\
\hline 0 & 175.53 & 210.10 & 173.72 & 173.79 & 173.82 & 173.74 & 173.85 \\
5 & 153.25 & 178.91 & 151.46 & 151.52 & 151.54 & 151.57 & 151.57 \\
10 & 122.16 & 143.39 & 120.39 & 120.44 & 120.46 & 120.48 & 120.48 \\
15 & 88.88 & 98.68 & 87.14 & 87.18 & 87.19 & 87.20 & 87.21 \\
20 & 54.40 & 47.97 & 52.64 & 52.71 & 52.72 & 52.73 & 52.73 \\
\hline
\end{tabular}

Table 6.10 Different eye resolutions in distance estimation

\begin{tabular}{|cccllllll|}
\hline \multicolumn{7}{|c|}{ Mean estimated speed [SEE], with different eye resolution } \\
\hline $\begin{array}{l}\text { Time } \\
\text { step(s) } \\
{[\mathrm{T}]}\end{array}$ & $\begin{array}{l}\text { Mean Actual } \\
\text { Speed (m/s) } \\
{[\mathrm{MAS}]}\end{array}$ & $\begin{array}{l}\text { Mean Estimated } \\
\text { Speed (m/s) } \\
\text { [Driver subject] }\end{array}$ & $\begin{array}{l}\text { col.row } \\
50 \times 25\end{array}$ & $\begin{array}{l}\text { col.row } \\
50 \times 50\end{array}$ & $\begin{array}{l}\text { col.row } \\
100 \times 50\end{array}$ & $\begin{array}{l}\text { col.row } \\
100 \times 100\end{array}$ & $\begin{array}{l}\text { col.row } \\
\text { 300x100 }\end{array}$ \\
\hline 2.5 & 4.46 & 6.25 & 4.4563 & 4.4561 & 4.4561 & 4.4559 & 4.4559 \\
7.5 & 6.22 & 7.10 & 6.2193 & 6.2191 & 6.2191 & 6.2189 & 6.2189 \\
12.5 & 6.66 & 8.94 & 6.6559 & 6.6557 & 6.6557 & 6.6555 & 6.6553 \\
17.5 & 6.90 & 10.14 & 6.8967 & 6.8964 & 6.8964 & 6.8962 & 6.8962 \\
\hline
\end{tabular}




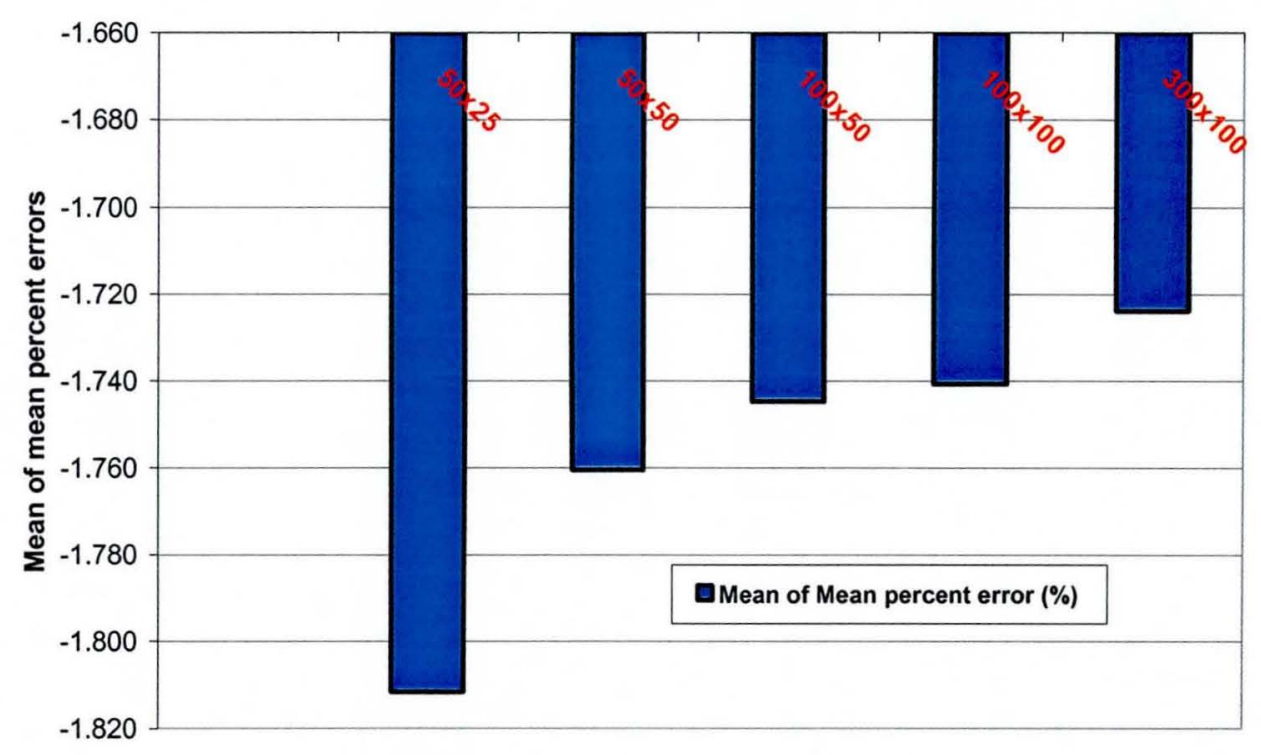

Figure 6.10 Eye resolution and accuracy error for speed perception

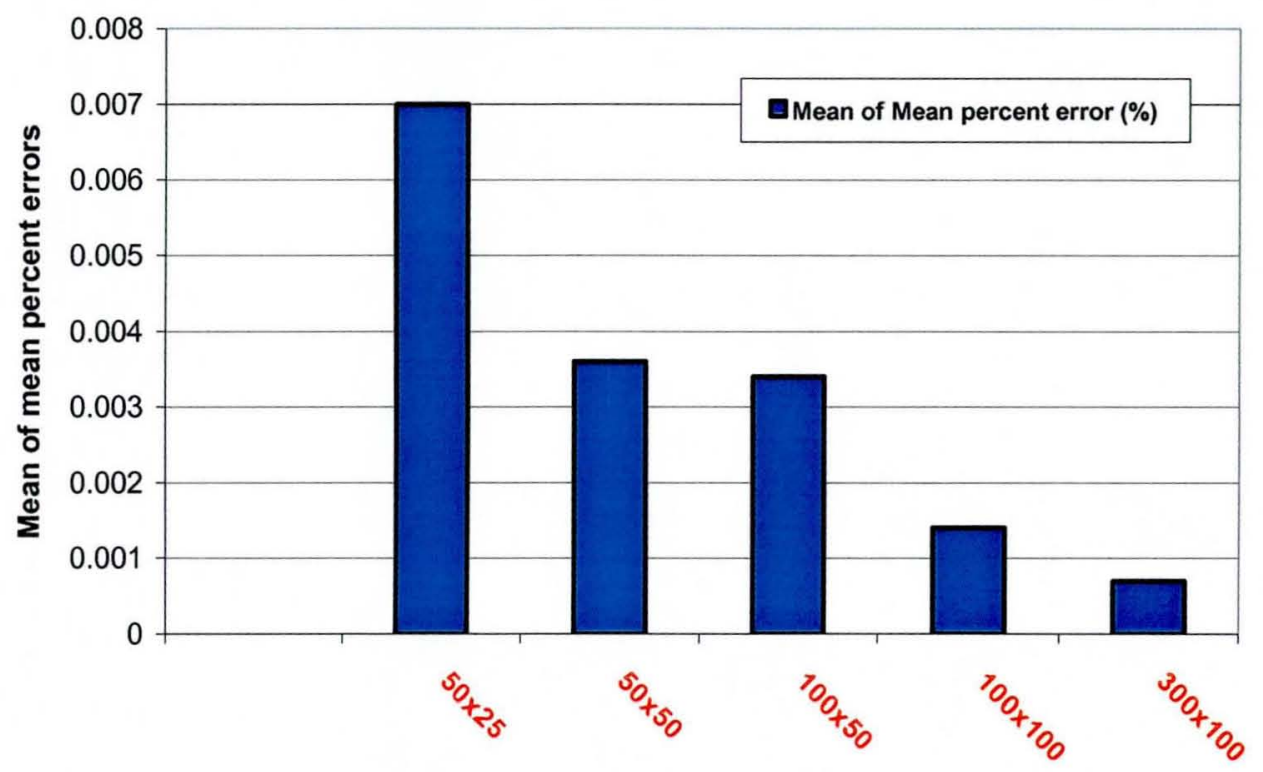

Figure 6.11 Eye resolution and accuracy error for speed perception

\subsubsection{Validation of Intelligent Virtual Driver Behaviour}

Driver behaviour models are difficult to evaluate due to a number of reasons. Firstly, there is usually a lack of microscopic data to calibrate the many different model parameters. Secondly, as a result of the variability of decision making between individuals, it is difficult to identify and evaluate common behaviour since, for example, different drivers encountering the same situation will tend to make different decisions. Thirdly, the evaluation of driver behaviour models usually requires a large number of model parameters. Considering these issues, evaluation of how well SD- 
SIM produces intelligent driver behaviour has been limited to the basic model of lane changing. It is important to note that, although the validation exercise of intelligent driver behaviour is focussed on this behaviour, in fact this in turn is enabled by a range of lower level behaviour such as distance and speed perception, collision avoidance, overtaking, slowing down, etc.

\subsubsection{Lane Changing}

The evaluation of SD-SIM's lane changing behaviour involved an assessment of virtual driver lateral displacement compared to real vehicle trajectory. From experiment 1 (motorway experiment), a section of the videos (1440 frames) involving lane changing were analysed to obtain the trajectories of a lead and following vehicles. The variables obtained from these data included headways between the two vehicles, acceleration, speeds and lane changes. The average values (over a period of time) of these variables were calculated. In addition, the desired or preferred following distances for both Police drivers were obtained from short interviews. They indicated their preferred time headways were according to the stipulated 2 seconds rule of thumb. This was then used to calculate their preferred distance headways. To obtain the preferred distance headway for each virtual driver agent in SD-SIM, the mean speed calculated from the vehicle trajectory was multiplied with the average time headway of 2 s. In summary, lane changing was implemented using the following:

- Two virtual driver agents i.e. lead and following were programmed in SD-SIM.

- The lead vehicle was programmed with an initial speed value of $28.16 \mathrm{~m} / \mathrm{s}$ with initial acceleration of $0.45 \mathrm{~m} / \mathrm{s}^{2}$.

- The initial speed of the following driver agent was also defined as $28.16 \mathrm{~m} / \mathrm{s}$ with initial acceleration of $0.89 \mathrm{~m} / \mathrm{s}^{2}$.

- Both vehicles initially occupied lane 2 with initial separation between the lead and following vehicle at a position corresponding to the mean separation distance obtained from the video data of $16.09 \mathrm{~m}$.

- Preferred following distance headways for each driver agent were calculated as described above.

- $\quad$ The rule to change to preferred lane was weighted for driver 2 as 0.9 . 
Table 6.14 shows the simulation results with driver 2 changing to lane 3 at a speed of $28.61 \mathrm{~m} / \mathrm{s}$. In addition, figure 6.13 compares lateral displacement of real video data and simulated driver lane changing behaviour. The results show a good match of lane changing profiles between simulated and real driving.

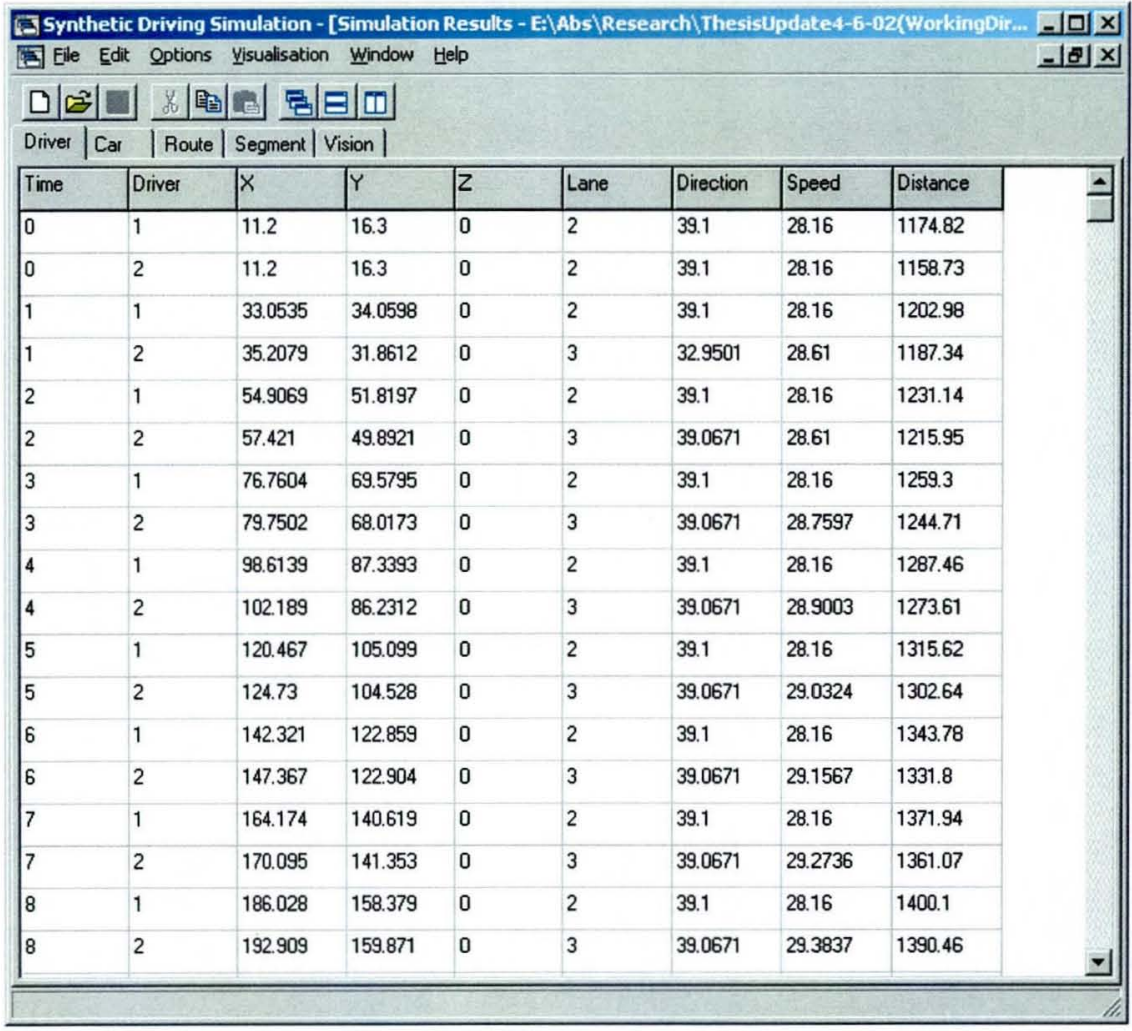

Table 6.14 Results of lane changing behaviour

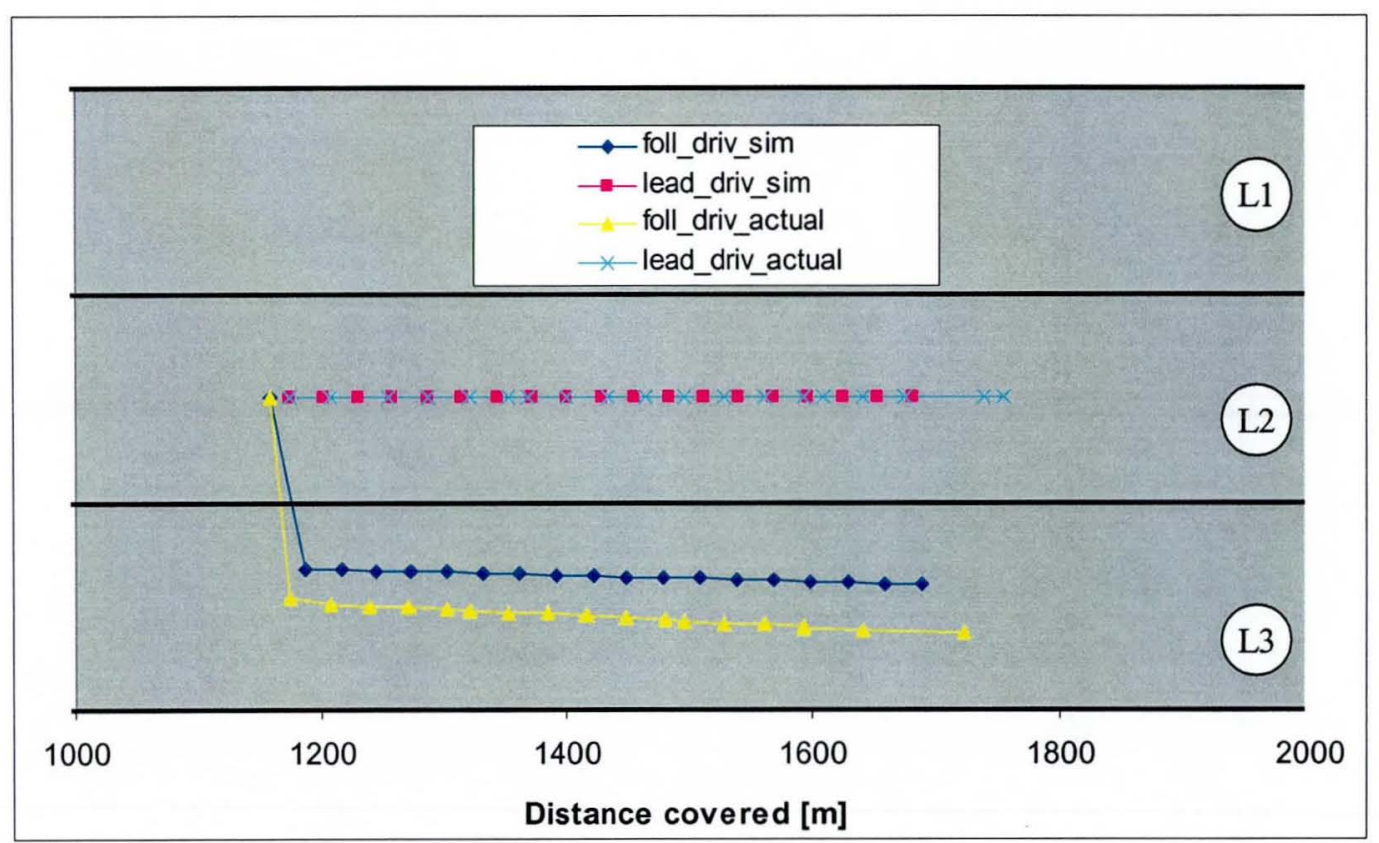

Figure 6.13 Comparison of vehicle trajectories 


\subsubsection{Discussion of Real World Scenarios}

The validation results presented in section 6.3.2 clearly demonstrate the vision model's capability to detect moving objects (vehicles) and estimate their positions and speeds. This allows sighted driver agents in the simulation framework to use perceived uncertain information rather than rely on accurate global knowledge to make intelligent decisions. This enhances the realism of SD-SIM over other traffic and driver simulations developed elsewhere. The accuracy calibration offers the opportunity to assess the degree of fit between SD-SIM's estimates and the measured data. The mean percent errors shown in figures 6.4 and 6.6 give an indication of performance that includes the effects of under- and overestimation of distances and speeds, but does not measure total error. In contrast, the RMS measure provides a cumulative total of the estimation errors, but does not reflect the difference between under- and overestimation. Hence both measures are of value here.

From the validation results it is apparent that the current version of the vision model is accurate in comparison to measured data, however it does consistently underestimate both distance and speed. The reason for this is the difference between actual distance and apparent depth, discussed in section 4.4.3.1. Also, instrument errors could have contributed to the consistent underestimation by the vision model. It should also be noted that the vision model performs significantly better than the driver subjects used in the experiments. This is plausible because human vision is incredibly poor at quantifying absolute values such as distance and speed, a widely recognised factor that contributes to many rear-end collisions. Regarding the static or dynamic nature of the experiments, it is also observable from figures 6.15 and 6.16 that human estimates improved in the dynamic estimate of speed compared to the static, 'stop and go' experiments. This is consistent with the fact that human perception in many situations is more sensitive to moving objects than stationary ones, since moving objects pose a greater danger to the observer.

Although the significant differences between the human and vision model estimates seem to suggest that the vision model is not a good representation of human driver vision, it is important to recognise that the model also facilitates future developments. For example, the model already provides a sound basis for filtering out vehicle and 
other objects in a simulation that are of little significance to a driver agent. The model also provides a framework for introducing other sources of uncertainty into visual perception and decision making, which are characteristic (models) of different types of human drivers. Furthermore, it should be noted that the main purpose of performing the experiments was to generate quantitative test data from real-life instruments and to assess the current performance of the vision model against these measuring devices. The benefit of additionally using human drivers in the experiments was that an initial crude comparison could be made between them and the vision model, allowing the inherent fuzziness in human perception to be considered within the further development of the vision model parameters.

The results in section 6.3.3.1 described lane changing behaviour. The driving data extracted from the motorway videos in experiment 1 has shown good agreement with simulated driver behaviour in lane changing. However, the lane changing evaluation has also proven difficult. Data was not available to calibrate gap acceptance due to the interaction of three or more vehicles. 


\subsection{Case Study: Real-Life Scenario Modelling}

SD-SIM is intended to be used as a tool to model microscopic interactions of the driver, vehicle and road network. As part of a current feasibility study, SD-SIM has been used to investigate a road traffic accident. In this project road accident data collected from crash investigations were defined as accident parameters within SDSIM's interface. Since SD-SIM provides an integrated framework with virtual drivers capable of perception-decision-action, parametric modifications to driver, vehicle and road environment can be made to quantify crash outcomes and produce animation visualisation. Although this is currently a Highway Agency research project, the current version of SD-SIM was used within the UK OTS (On-The-Spot) Accident study to analyse an accident situation through accident reconstruction. The typical steps in configuring SD-SIM for this kind of analysis involve:

- The crash investigator collected accident data for people, vehicles and the road network. This data included, travelling speeds obtained from skid marks, posted speed, separation distance after collision, driver behaviour obtained from witness statements, etc.

- The data was used to configure driver, vehicles and road network parameters using SD-SIM's interface.

- The crash investigator defined a time duration for which the simulation should be run.

- The simulation was run and it performed all calculations for the given situation. The data was displayed as quantified results.

- The investigator can choose to analyse this on a post process basis or produce a detailed animation visualisation.

\subsubsection{The Accident Scenario}

The scenario was obtained from a crash investigator's report. A Ford Sierra and Land Rover Freelander collided after travelling towards each other on a near-straight section of a single carriage way. Intending to overtake other traffic, the Sierra driver moved out into the path of the Freelander, and the vehicles collided. A scene plan is 
shown in figure 6.14. A number of crude estimates and other assumptions have been made for the road, along with the Sierra's and Freelander's approach.

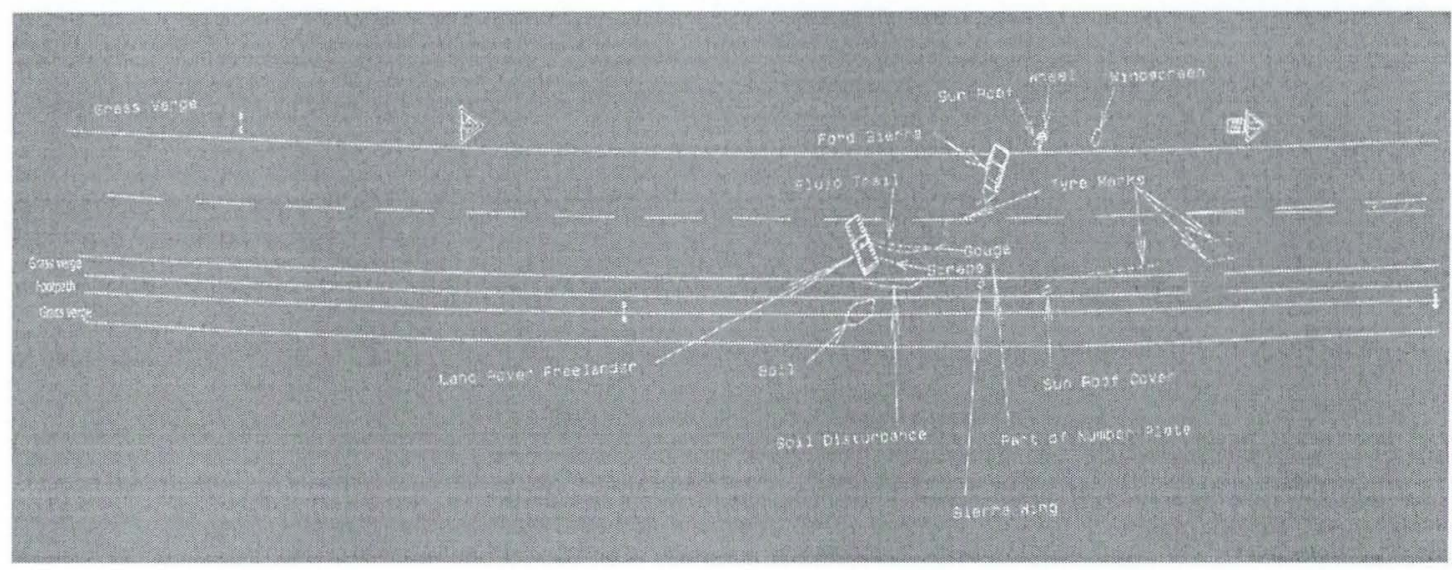

Figure 6.14: Accident scene plan

\section{The Road}

The road is a single carriageway, main road with the $60 \mathrm{mph}$ National speed restriction in place. The impact occurred on a Winter's evening, in the dark. There is street lighting which was illuminated. Dimensions for the highway, vehicle trace marks and vehicle final rest positions have been measured from the scale scene plan and are presented in table 6.15 .

\begin{tabular}{|c|c|c|c|}
\hline Description & $\begin{array}{l}\text { Plan reference } \\
\text { (see scene_plan_with_tets.doc) }\end{array}$ & $\begin{array}{l}\text { Distance taken } \\
\text { irom plan }(\mathrm{mm})\end{array}$ & $\begin{array}{r}\text { Estimated distance } \\
\text { on road }(m)\end{array}$ \\
\hline Length of road shown on plan & $\mathrm{H}$ to I & 338 & 135.2 \\
\hline Lane width (Sierra approach) & at $A$ & 11.4 & 4.6 \\
\hline Lane width (Freelander approach) & at $B$ & 10.5 & 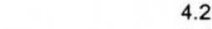 \\
\hline Verge width & at $\mathrm{C}$ & 3.4 & 1. \\
\hline Path width & at $\mathrm{D}$ & 3 & 1.2 \\
\hline Verge width & at $\mathrm{E}$ & 5 & 2.0 \\
\hline Length of white line & at $\mathrm{J}$ & 15 & 6.0 \\
\hline Distance between white lines & at $\mathrm{K}$ & 7.4 & 3.0 \\
\hline $\begin{array}{l}\text { Datum to point of impact as indicated by } \\
\text { "Gauge" }\end{array}$ & I to $M$ & 126 & 50.4 \\
\hline $\begin{array}{l}\text { Freelander's lateral position on approach, } \\
\text { as indicated by front off-side tyre mark } \\
\text { measured from median line on road }\end{array}$ & at $\mathrm{L}$ & 4.75 & 1. \\
\hline $\begin{array}{l}\text { Freelander displacement from first wheel } \\
\text { lock-up to impact }\end{array}$ & $L$ to $M$ & 76.5 & 30 \\
\hline $\begin{array}{l}\text { Freelander displacement from first wheel } \\
\text { lock-up to rest }\end{array}$ & $L$ to $F$ & 93 & 37 \\
\hline Rest position of Freelander from datum & I to $F$ & 142 & 56.8 \\
\hline Freelander length & published vehicle standard measures & & 4.3 \\
\hline Freelander width & published vehicle standard measures & & 1.80 \\
\hline Freelander height & published vehicle standard measures & & 1.7 \\
\hline Rest position of Sierra from datum & I to $M$ & 110 & 44 \\
\hline Sierra length & published vehicle standard measures & & 4.4 \\
\hline Sierra width & published vehicle standard measures & & 1.73 \\
\hline Sierra height & published vehicle standard measures & & 1.40 \\
\hline Vehicle separation at rest & $\mathrm{F}$ to $\mathrm{G}$ & 34 & 13 \\
\hline
\end{tabular}

Notes: 1 Datum point is lamppost shown at point I to far right of plan

2 All distances from the datum are measured parallel to the median line on the road

3 Vehicle locations are measured from their estimated centres of gravity

4 Distances are estimated to be accurate to $+/ .0 .15 \mathrm{~m}$, at best!?

5 Photo4.jpg is taken from approximately plan ref. $\mathrm{H}$

Table 6.15 Measurement of roadway, vehicle trace marks and vehicle rest positions 


\section{Sierra's Approach}

This is shown from left to right on the scene plan. Other road users reported that this driver had been driving erratically for some distance prior to the impact. He had steered the vehicle from kerb to median line on several occasions and made a series of erratic overtakes.

The approach can be seen over approximately $1.7 \mathrm{~km}$ as subsequently recorded on video. The driver will have negotiated a very gradual right hand curve for $350 \mathrm{~m}$, followed by a straight section for $700 \mathrm{~m}$, the collision then took place on a section that very slightly curves to his left for some $650 \mathrm{~m}$ to the point of impact. The speed of approach obtained from witness and damage evidence suggest 60 to $75 \mathrm{mph}(96-120$ $\mathrm{km} / \mathrm{h})$.

\section{Freelander's Approach}

This is shown from right to left on the scene plan. Little is known about the driving style of the Freelander upon approach. The driver claims not to have seen the Sierra move out into his path, and subsequently took late avoiding action that included heavy braking. Tyre marks were caused by wheel lock up on braking, and these allow us to place the vehicle accurately on the road at that point. The approach speed obtained from witness and damage evidence suggests 50 to $65 \mathrm{mph}(80-104 \mathrm{~km} / \mathrm{h})$.

\subsubsection{Parametric Modelling Procedure}

Due to the current limitations of SD-SIM in terms of modelling roads with curves the scenario was simulated for a segment of the road that was $1350 \mathrm{~m}$. The overall road width extracted from table 6.14 was set in SD-SIM at $8.8 \mathrm{~m}$, giving a lane width of 4.4 $\mathrm{m}$. Modelling vehicle dynamics in SD-SIM is also currently limited therefore, skid marks produced due to wheel locks by the freelander have not been considered. The dimensions of the Sierra and Freelander were obtained from published vehicle standard measures i.e. Parker's Guide.

To capture the reported erratic driving of the Sierra driver, the author has assumed low weights for maintaining position within lane and maintaining short headways, e.g. 0.1 and 0.2 respectively. This process was achieved by trial and error. Since very little 
is known of the driver of the Freelander, various assumptions relating to lane position maintenance, and conservative choice of parameter values such as travelling and preferred speeds have been made. The accident report also claims that the Freelander driver did not see the Sierra driver changing lanes. There could be speculative reasons for example, lack of driving attention due to some kind of distraction or possible poor vision. To demonstrate the vision effects, a very crude assumption was made giving the driver's low visual resolution. This assumes that poor vision correlates to low resolution or less rays emanating from the eyes. To demonstrate the interactions of other drivers as confirmed by the investigator's report, an extra driver travelling in the same direction as the Sierra has been introduced. This driver has been defined with default parameter values.

Figure 6.15 illustrates parametric configuration of the scenario in SD-SIM. Person 1 and 2 refer to the drivers of the Sierra and Freelander respectively. The figure shows the initial potions of the vehicles.

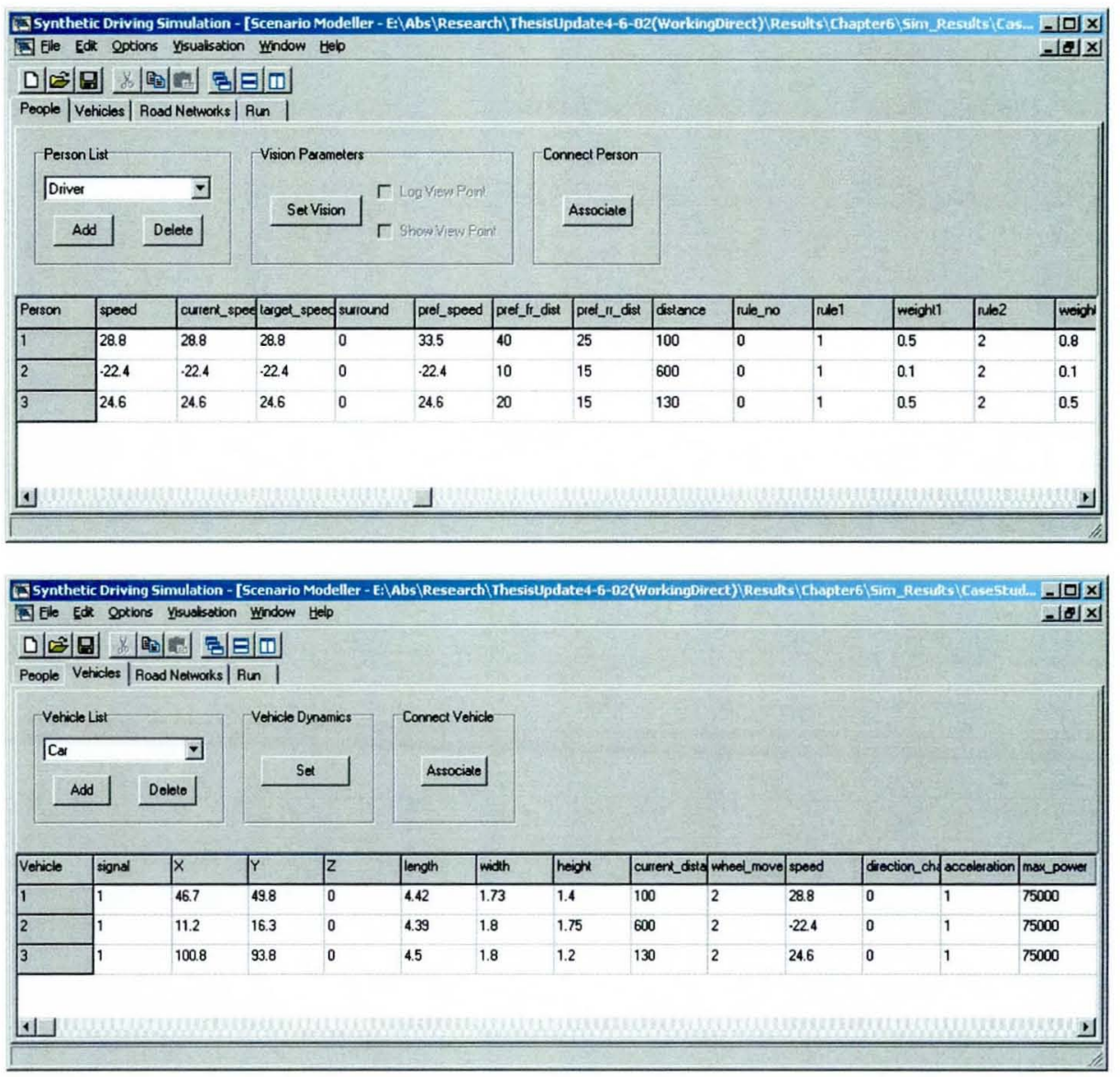

(a)

Figure 6.15 Defining accident variables in SD-SIM (a) Driver parameters (b) vehicle 


\subsubsection{Analysis of Road Accident Data}

Figure 6.16 shows the trajectories of the vehicles during the incident between the Sierra and Freelander. The figure shows the Sierra driver overtaking and moving into the path of the Freelander. This resulted into a collision deflecting the Freelander's trajectory.

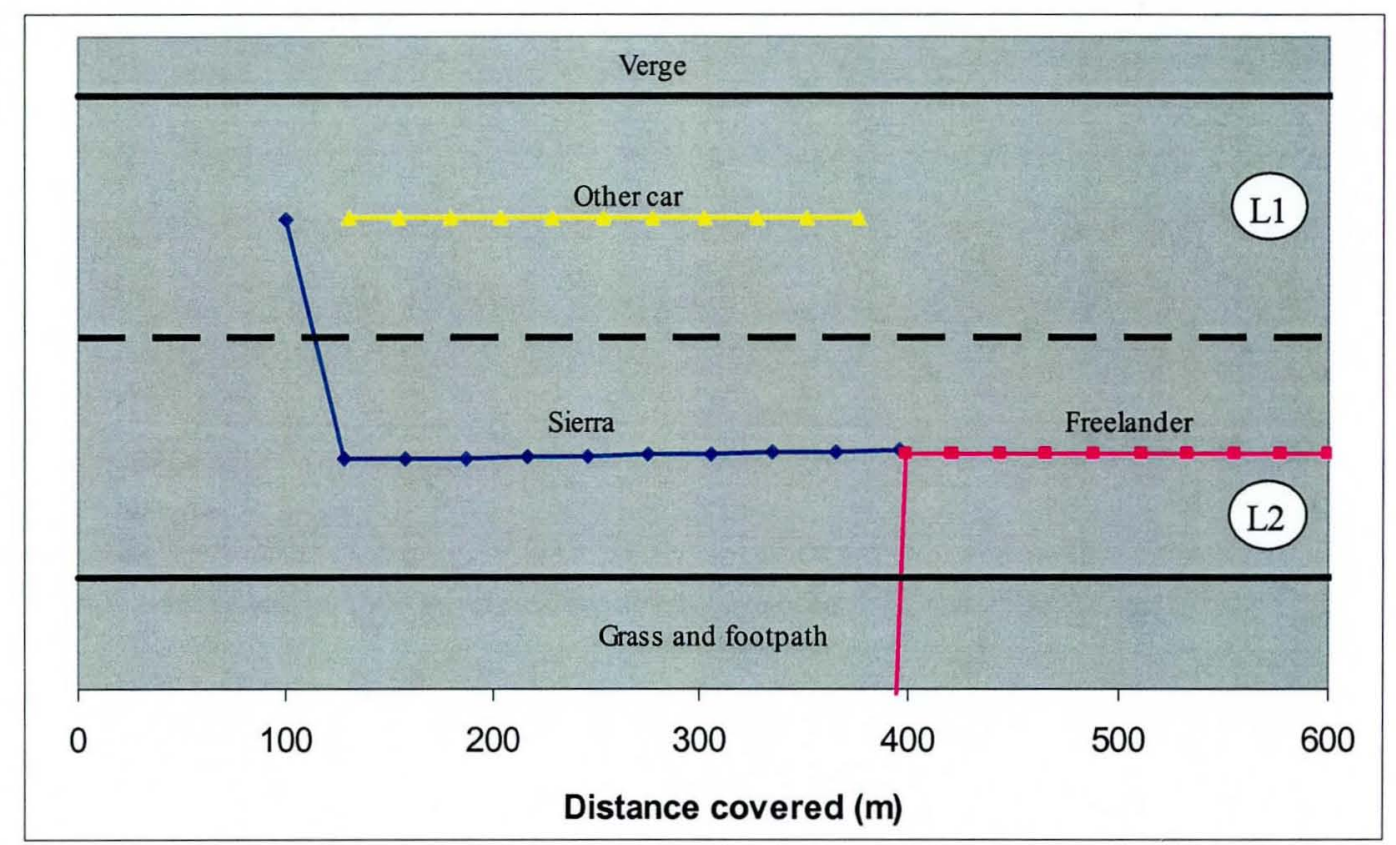

Figure 6.16 Vehicles trajectories during the incident

Applying equation 6.5, the time-to-collision information can be calculated. This is presented in table 6.16, along with the relative distance and speeds between the Sierra and Freelander.

\begin{tabular}{ccc}
\hline Relative distance $[\mathrm{dx}][\mathrm{m}]$ & Relative speed $[\mathrm{dv}][\mathrm{m} / \mathrm{s}]$ & Time-to-collision[TTC][s] \\
\hline 500.000 & 51.200 & 9.77 \\
448.800 & 51.200 & 8.77 \\
397.600 & 51.200 & 7.77 \\
346.156 & 51.444 & 6.73 \\
294.484 & 51.673 & 5.70 \\
242.596 & 51.888 & 4.68 \\
190.505 & 52.091 & 3.66 \\
138.222 & 52.283 & 2.64 \\
85.759 & 52.463 & 1.63 \\
33.125 & 52.634 & 0.63 \\
\hline
\end{tabular}


The TTC information has practical implications to the traffic investigator who would otherwise have to derive this information from skid marks. Figure 6.17 compares different view points of the accident scenario. The first picture shows a video re-run of the accident from the Ford Sierra's approach. A similar view is shown in the picture on the top right. The bottom left and right show views from another approaching car and aerial view. One of the main benefits of SD-SIM and its visualisation capability is that accident data can be evaluated safely and visualisation provided without the risky situation shown in the top left picture - the reconstruction of the accident during traffic.

However, the current version of SD-SIM has several limitations regarding the reconstruction of accidents. Firstly, parametric modelling involves manually tuning the rules and weights to achieve the desired behaviour. As mentioned earlier this is achieved mainly by trial and error and can become very time consuming. Secondly, SD-SIM cannot handle vehicle deformation after crash. This is because the current version of the software lacks a realistic vehicle dynamics model.
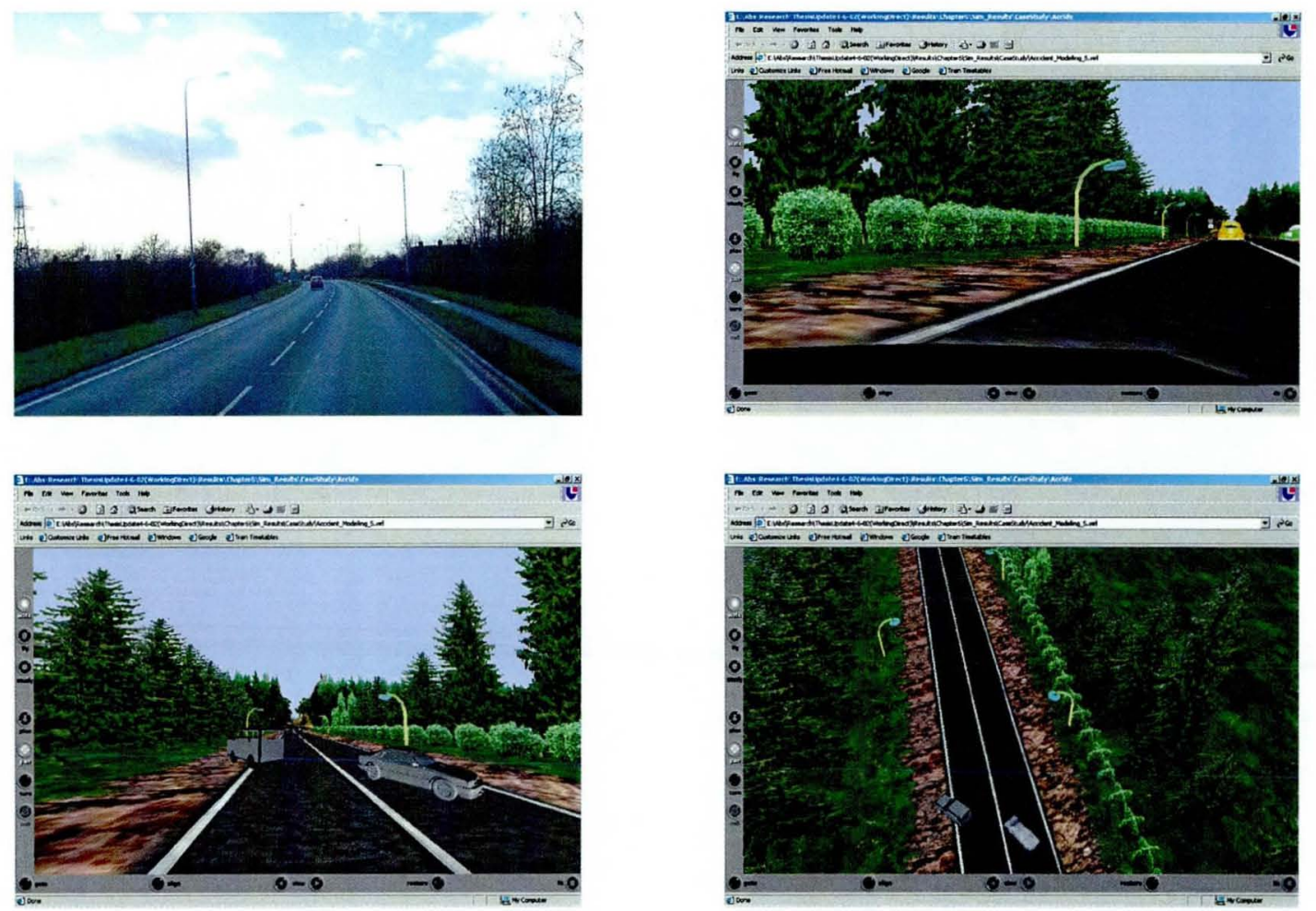

Figure 6.17: Multiple viewpoints of accident scenario 


\subsection{Chapter Summary}

This chapter has described the evaluation of SD-SIM from two perspectives. The first involved assessing the functional capabilities of SD-SIM in terms of its design requirements. This demonstrates performance measure of SD-SIM. The second perspective involves describing the potential application of SD-SIM, demonstrated by a case study of accident data modelling.

Although it is clear that SD-SIM compares favourably with published data, there are several critical points worth mentioning. Firstly the use of comparative studies is often dependent on understanding the underlying assumptions of the related work that is being compared. In some cases, this is not always possible since data or experimental procedures are not fully described or available. Therefore the author has had to make various assumptions based on information gleaned from the literature to provide parity in comparison. Secondly, comparative studies tend to employ different techniques and methods of data analysis. The variability in these methods could also be sources of errors. However, comparative studies provide the opportunity to benchmark/calibrate the work reported in this thesis against similar research work and help identify areas of further research. At this stage of evaluation of SD-SIM using comparative studies has been termed 'low-level'. The 'high level' evaluation has been achieved through the designed and conduction of real-life experiments to calibrate SD-SIM's algorithms and functionality. This has also showed favourable comparisons of results produced from both in the literature and experimental data. The evaluation of SD-SIM based on real-life accident data has provided opportunity to assess scenario modelling and quantitative estimates of driver behaviour in these situations. 


\section{CHAPTER 7}

\section{CONCLUSIONS AND FUTURE RESEARCH}

\subsection{Introduction}

This section presents the conclusions drawn from the work undertaken in this research. The primary contributions of this thesis are the design, development and validation of driver vision and visual perception as well as the development of SDSIM framework to demonstrate intelligent virtual driver interactions and traffic flow. The SD-SIM modelling framework and the incorporated visual perception model are both outputs from this research. SD-SIM also incorporates a previously developed driver decision making model (Wood and Arnold 1997). Sections 7.2 and 7.3 present the primary contributions of the research, whilst section 7.4 summarises the conclusions from the research. Recommendations for further work are discussed section in 7.5 .

\subsection{Driver Vision and Visual Perception}

Explicit access to the calculated positions and velocities of other driver agents in a simulation is the most unrealistic approximation of driver vision in many contemporary driving simulation frameworks. To remove this 'perfect global vision', this work has introduced a new vision model, based on computer graphics concepts and simple biological assumptions, into a microscopic traffic simulation tool. This has also established a framework for introducing various cognitive models. The implementation of driver vision has enhanced the realism of simulated driver decision-making and behaviour, which leads to improved simulation of autonomous driver interactions and traffic flow.

\subsection{Autonomous Driver Agents and Emergent Traffic Flow}

In demonstrating autonomous driver agent interactions, the author has developed SDSIM to allow the user to configure different scenario parameters for individual driver agents as well as vehicle and road network components and run the simulation to 
observe the behaviour of the driver agents as a result of these parameters. The approaches used to support the framework for autonomous virtual driver agents and emergent traffic flow are derived from Artificial Life (ALife), Artificial Intelligence (AI), Agent Based Simulation and Driver Psychology. Each driver agent in SD-SIM contains knowledge and a decision-making mechanism, both of which are based on heuristics. Emergent traffic flow occurs due to interactions between individual agents in the simulation. These interactions are achieved by endowing virtual driver agents with the capabilities to perceive their environment, to support their decision making and actions.

\subsection{Conclusions from the Research}

A summary of the conclusions derived from the research are as follows:

(i) A new vision model, SEE, has been developed and successfully integrated to augment a previously developed model of driver decision making enabling visual detection of other vehicles and perception of their behaviour. This vision model has removed the unrealistic availability of 'perfect knowledge' concerning the positions and velocities of vehicles and has contributed to the realism of simulated driver decision making and behaviour.

(ii) A novel microscopic traffic simulation SD-SIM tool, based on object oriented design and $\mathrm{C} / \mathrm{C}++$ implementation paradigms have been developed. This tool is unusual in that it makes a clear distinction between the three main components of driving i.e. the driver, the vehicle and the road network. In this framework, the characteristics of human drivers are abstracted and packaged within intelligent virtual driver agents capable of perceiving their environment, making decisions based on what they 'see' and taking appropriate driving actions. SDSIM provides facilities to allow the user to configure different driving scenarios and observe the interactions between virtual driver agents in their traffic environment.

(iii) In order to evaluate SD-SIM's algorithms and functionality, driving experiments have been designed and conducted with the support of 
Leicestershire Constabulary, Traffic Division. Measurement data relating to instruments, human subjects' perception of distance and speed was collected and formed the main basis for an initial calibration and validation of SEE. SEE showed a consistent performance with instrument data.

(iv) In addition to assessing the validity of SD-SIM, its potential application to understanding accident causation factors through the modelling and analysis of accident data has been demonstrated through a case study.

(v) A thorough and critical review of the literature on vision, driver behaviour models and traffic simulation has been carried out. This has highlighted the current gaps in which driver vision capability in contemporary driving simulation frameworks is either completely ignored or abstracted in an unrealistic manner. Furthermore, knowledge gained from the review has proven useful in formulating fundamental concept of this thesis as well as the subsequent computational framework that has been developed.

(vi) Related fields of Computer Graphics, ALife, Agent technology and AI along with some of their allied techniques have been exploited to support efficient model abstraction whilst aiming to minimise software complexity.

\subsection{Recommendations for Further Work}

The work presented in this thesis is by no means exhaustive and recommendations for future work are necessary. These recommendations are intended to enhance and extend the overall future functionality and realism of SD-SIM.

\subsubsection{Advanced Vehicle Dynamics Model and Driver Actions}

To improve realism in terms of the forces experienced by driver agents whilst driving their vehicles, a more advanced vehicle dynamics model is required to replace the current deterministic rule based model. The intention is to incorporate a physically based vehicle model of the form commonly used in vehicle dynamics simulation (Sayers 1998) and is described by Gordon in (Dumbuya et al 2002b): Such a model 
places limitations on vehicle manoeuvrability due to factors such as vehicle inertia, limited engine power, limited friction between the tyre and road, dynamic changes in vertical tyre loads, etc. Limitations also arise from driver skills, which are sometimes crucial in accident causation; a skilled driver can recover from a skid or spin, regaining control of the vehicle, whilst a novice is more easily confused by unexpected vehicle behaviour.

Introducing more realistic vehicle behaviour implies a need for a more sophisticated approach to the decision-action modelling within the Intelligent Virtual Driver. Currently the IVD operates at a high 'conscious' level based on the logical processing of traffic and driver intentions. Translating such desired actions into vehicle responses requires both open-loop and closed-loop activity at several levels. For example, once a decision has been made to attempt an overtaking manoeuvre, feedback from the traffic environment can countermand this decision, e.g. when new visual input indicates the danger of an accident. If the initial decision stands, the driver plans a new speed and path for the vehicle. An incredibly skilled driver could perform this 'with his eyes shut' (open-loop control) but in reality corrections (closed-loop control) are needed - extra throttle or slight changes in the steering for example. The more familiar the driver is with the car, the more smoothly he or she will drive, and the less feedback is required. At a lower level still, some control actions are very much subconscious; reactively, the driver will 'hit the brakes' if a child suddenly emerges from behind a parked car, or pro-actively a skilled driver will use opposite lock steering to correct incipient loss of control in spin. These actions are generally learned through driver training, and represent low level conditioned reflexes. These various levels of behaviour have been described in various papers (see for example (Weir and McRuer 1970)) and a flexible and generic driver model that incorporates path planning and feedback control (at the conscious level) has been described in (Gordon et al 2002). It should be clear from the above that the use of physically based vehicle modelling is a challenging development that is fundamental to the integration of an agent-based traffic model with real-life accident causation factors. Some of these issues are being addressed in a recently started research project (Wood et al 2003). 


\subsubsection{Realistic Road Networks and Traffic Environment}

The current SD-SIM road network structure is a very simple representation of 3 lane motorway, although it is possible to configure it (with difficulty) for 2 lane traffic. To improve this, a means of providing road geometry and definition of logical information is needed. In developing new road geometry, attention should be given to the different types of junctions or roundabouts e.g. merging and diverging junctions, roads with curves, elevation, crossroads as well as street furniture such as traffic lights. This will provide a more rich and realistic environment and extend the application of SD-SIM to a broad range of traffic scenarios. Although the current version of SD-SIM provides extensibility for road networks due to the advantages of the adopted object oriented approach, use could be made of existing specialist road network software like, AutoRoad ${ }^{\circledR}$ (Sabeur and Rommelfanger 2000), EVARISTE RESEAU $^{\circledR}$ (Charles and Espié 2000).

\subsubsection{Enhancement of Vision Model using Animat Vision}

The current vision model can be enhanced by supplementing binary (grey) images with colour images as well as incorporating foveal and saccadic abilities using animat vision algorithms. Colour perception will enhance image tracking and occlusion information processing. In addition, perception capability based on more robust biological systems rather than simple assumptions and heuristics should provide a more powerful vision paradigm useful for photorealistic rendering and analysing of synthetic environment. An example of this approach is demonstrated in (Rabie and Terzopoulos 2000).

Another challenge of enhancing the driver vision model is the development of algorithms for rear vision allowing virtual driver agents to look at side and rear view mirrors. At the moment only forward looking vision has been implemented and although a wider field of view can be defined to compensate for vehicles within the peripheral view, in order to be able to study for example drivers' visual scanning pattern while driving using SD-SIM, this capability would have to be added.

The current vision model is computationally intensive due to the ray tracing technique employed. Although it has been shown that improvement in object detection is a 
direct consequence of higher eye resolution, this impinges greatly on computational cost and time. Furthermore, since each driver can have vision, increasing computational time currently limits the number of driver agents that can populate the simulation environment. One way to improve the computational efficiency of the vision model and the overall performance of SD-SIM is to use a computer with a much more powerful processor. This is not a very big problem in the current state of hardware technology where the processors have become more powerful compared to the fall in their price.

\subsubsection{Improving Driver Decision Making}

Driver decision making relies on rules which are assigned weights. This process is limited in two ways; it is a manual and laborious task of configuring rule parameters for particular traffic investigations. In addition, these rules do not produce learning capabilities. Therefore, further work will be required in two directions. Firstly, automating the tuning of decision parameters and secondly, incorporating learning functionality. Both tasks can be achieved by evolutionary or fuzzy-neural techniques. Evolutionary techniques are useful optimisation methods used to evolve solution to complex search problems. For example using GA (Genetic Algorithm - a common evolutionary technique), optimal decisions can be reached automatically and easily by the algorithm selecting 'fitter' rule(s) in a population of competing rule candidates. The fitter rule candidates are then evaluated and used in the next generation and the whole process continues.

Furthermore, the incorporation of learning in the IVD could also greatly improve or remove completely some of the prescriptive nature of driver decision and actions. Using fuzzy neural techniques, the description of rules can take a very vague format, whilst neural learning strategies ensure that training data sets are accommodated in a general way to enable 'unsupervised' and 'adaptive' learning through tuning fuzzy rules and parameter optimisation. 


\subsubsection{A Brain Model for Virtual Driver Agents}

Section 7.5.4 discussed possibilities of introducing learning abilities for the IVD. However, to approximate driver agents' capability to their human counterparts would require modelling of low-level brain function. The ability to model selected brain function within virtual driver agents is a novel approach and one that the author is not currently aware exists. Although this is a challenge, endowing driver agents with low level brain function may allow representation of more subtle effects of brain behaviour in different driving related circumstances e.g. substance abuse and tiredness. For example, modelling brain function could supplement the current methods implemented for the driver vision and decision making. For example, visual information processing could be extended to a model of the visual cortex enabling realistic representation and processing of visual information.

Pursuing the idea of a brain model has already being attempted. Bond (1999) describes a brain model of primate brain architecture. This model is developed for intelligent social agents and is based on an extensive study and review of neuroanatomical connections and functional involvements of cortical regions. Virtual driver agents in SD-SIM could benefit from a similar model. One possible approach is to study human brain function in the laboratory, whilst executing driving tasks under different driving conditions. Drivers' brain function can be mapped using Functional Magnetic Resonance Imaging technique (FMRI), which allows parts of the subject's brain, activated by physical sensation or activity e.g. sight of simulated traffic flow, sound, etc. to be scanned. The MRI images are analysed and the understanding gained from this can be used to build a realistic brain model for virtual driver agents.

\subsubsection{Further Validation Using Motorway Driving Experiments}

The validation work carried out in chapter 6 was aimed at evaluating key features of SD-SIM. However, as indicated in the discussion of results in section 6.3.4, the validation and analysis process was limited by the number of the subjects and the fact that the experiments were conducted in a racetrack. In addition, data collected to evaluate SD-SIM's lane changing model were also limited. It is therefore important to extend this validation exercise using data collected from real life motorway driving. 
This work will follow from previous work done by (Brackstone et al 1999) and will involve assessing a large numbers of subjects' ability to perceive headways in car following and lane changing in a motorway situation.

\subsection{Potential Applications of SD-SIM}

This thesis has shown that the objectives and requirements for developing SD-SIM have successfully been achieved. In so doing, several contributions have been made in the areas of visual perception modelling, driver behaviour models and overall understanding of traffic flow. In concluding this chapter it is useful to explore potential applications of SD-SIM.

- SD-SIM can be used for more detailed prediction and analysis of traffic/accident situations e.g. replication of accident scenarios. As the simple case study shows (section 6.4), the traffic investigator can define accident data within SD-SIM to recreate the same scenario that led to an accident and replay the scenario from different multiple views. In the same way, traffic prediction of the different variables is also possible by combining different parameters to observe their impact to traffic causation.

- Applying the agent framework to autonomous driving vehicles. In this respect, agents will receive active vision information from the environment and make decisions based on this information in terms of the objects detected and other environmental properties extracted. The agent will then execute control actions to steer the vehicle allowing the vehicle to drive on the road autonomously.

- In addition to traffic/accident analysis, psychological factors that influence general driving and traffic flow can also be evaluated. For example, perception of headways can be used to investigate rear end collisions.

- SD-SIM can also make a significant contribution to driver training and testing. For example studying hazard perception by recreating different scenarios and observing the responses of driver subjects. 
- With a well developed brain model, potential use to investigate issues such as tiredness, substance abuse and defective vision in the context of driving can be possible. For example, the performance of virtual driver agents under the influence of simulated chemicals can be assessed safely.

- SD-SIM can be used with virtual reality so that human can interact with agents. In this application, SD-SIM would become part of the artificially intelligent traffic generation component of a fixed or motion based driving simulator. This will allow different experiments to be designed involving for example, driver subjects seating in the simulator and interacting with the virtual driver behaviour.

- Road and junction design and analysis. The integration of the driver, the vehicle and road network within a single framework provides the opportunity to analyse in detail any one of these components. For example, the behaviour of drivers to junction modification or the effect of road geometry on driving can be simulated to provide engineers with useful information.

- Modelling of vision disorder and their influence on driver behaviour. SD-SIM vision perception model can be extended to include more realistic biological representation so that modelling at the cellular and neural transmission level of vision can provide insight into vision disorders. 


\section{REFERENCES}

Ahmed, K. I., Ben-Akiva, M. E., Koutsopoulos, H. N., Mishalani, R. G., 1996, Models of freeway lane changing and gap Acceptance. In J.-B Lesort (eds) Transportation and Traffic Theory (New York: Elsevier Publishing), pp. 501-515.

Albus, J. S., 1991, Outline for a theory of intelligence. IEEE Transactions on Systems, Man and Cybernetics, 21 (3): 473-509.

Allen, R. W., Rosenthal, T. J., Aponso, B. L., Klyde, D. H., Anderson, F. G., and Chrstos, J. P., 1998, A low cost PC based driving simulator for prototyping and hardware-in-the-loop applications. In Society of Automotive Engineers, SAE Paper No. 98-0222, Special Pub. 1361, Detroit, MI, pp. 35-47.

Allen, R. W., Rosenthal, T. J., Klyde, D. H., and Chrstos, J. P., 2000, Vehicle and tire modelling for dynamics analysis and real-time simulation. In Society of Automotive Engineers, SAE Paper 2000-01-1620, Warrendale, PA, pp. 1-22.

Allen, W. R., Rosenthal, T. J., and Hogue, J. R., 1996, Modelling and simulation of driver/vehicle interaction. In Society of Automotive Engineers, SAE Paper 960177, Detroit, MI, pp. 26-29.

Anderson, J.R., 1996, ACT: A simple theory of complex cognition. American Psychologist, 51(4): 355-365.

Arnold, J., 1997, An automata based simulation of co-operative decision making, MPhil. Transfer Report, Department of Manufacturing Engineering, Loughborough University, Loughborough, September 1997.

Artz, B. E., 1995, An analytical road segment terrain database for driving simulation, In Proceedings of Driving Simulation Conference (DSC'95), Sophia Antipolis, France, pp. 274-184, September 12-13, 1995. 
Bailey, A. C., 2000, Advancements in logical road network design for the Leeds Driving Simulator, In Proceedings of Driving Simulation Conference (DSC 2000), Paris, France, pp. 257-265, September 6-8, 2000.

Barlovic, R., Esser, J., Froese, K., Knospe, W., Neubert, L., Schreckenberg, M., and Wahle, J., 1999, Online traffic simulation with cellular automata, In K. Beckmann, W. Brilon, H. Wallentowitz and M. Schreckenberg (eds), Proceedings of the FVU Workshop (Aachen Germany: Springer Heidelberg), pp. 117-134.

Bayarri, S., Fernandez, M., and Perez, M., 1996, Virtual reality for driving simulation. Communication of the $A C M, 39$ (5): 72-76.

Ben-Akiva, M., Bierlaire, M., Koutsopoulos, H. N., and Mishalani, R., 1998, DynaMIT: a simulation-based system for traffic predication and guidance generation, In Proceedings, TRISTAN III, San Juan, Porto Rico, pp 1-14, June 17-23, 1998.

Bergenti, F., and Poggi, A., 2000, Exploiting UML in the design of multi-agent systems, In A. Omicidi, R. Tolksdorf, F. Zambonelli (eds) Engineering Societies in Agents World: Lecture Notes on Artificial Intelligence (Berlin: Springer-Verlag), pp. 106-113.

Blumberg, B. M., 1997, Go with the flow: synthetic vision for autonomous animated creatures, In Proceeding of the First International Conference on Autonomous Agents, New York, ACM, pp. 538-539, February 5-8, 1997.

Boer, E. R., 1999, A statistical model of looming detection, In A.G. Gale, I.D. Brown., C.M. Haslegrave, and S.P. Taylor (eds.) Vision in Vehicles VII (Amsterdam Netherlands: Elsevier Science Publisher B.V.). pp. 319-327.

Bond, A. H., 1999, Describing behavioral states using a system model of the primate brain. American Journal of Primatology, 49: 315-388. 
Booth, M., Cremer, J. and Kearney, J, 1993, Scenario control for real-time driving simulation. In Proceedings of the fourth Eurographics Animation and Simulation Workshop, Barcelona, Spain, pp. 103-120, September 4-5, 1993.

Bordeux, C, Boulic, R. Thalmann, D. 1999, An efficient and flexible perception pipeline for autonomous agents. EUOGRAPHICS, 18 (3): 23-30.

Brackstone, M., and McDonald, M., 2000, Car following: an historical review. Transportation Research Part F, 2 (4): 181-196.

Brackstone, M., McDonald, M., and Sultan, B., 1999, Dynamic behavioural data collection using an instrumented vehicle, In Transportation Research Record 1689, TRB National Research Council, Washington DC, pp. 9-17.

Brilon, W., and Wu, N., 1998, Evaluation of cellular automata for traffic flow simulation on freeways and urban streets. Stadt Region Land, Institut für Stadtbauwesen der RWTH Aachen, Heft 66, S. pp. 111-117.

Brown, R. H., 1955, Velocity discrimination and the intensity-time relation. Journal of the Optical Society of America. 43 (3): 189-192.

Cameron, G., Wylie, B. J. N., and McArthur, D., 1994, Paramics - moving vehicles on the connection machine, In Proceeding, Supercomputing, IEEE Computer Society $A C M$, Washington DC, USA, pp. 291-300, September 13, 1994.

Cantoni, V., Levialdi, L., and Roberto, V., 1997, Artificial vision: image description, recognition and communication (San Diego CA: Academic Press), pp 3-191

Case, K., Porter, J. M., and Bonney, M. C., 1990, SAMMIE: a man and workplace modelling system, In W. Karwowski, A. Genaidy and S. Asfour (eds), Computer Aided Ergonomics: A Researchers Guide (London: Taylor and Francis Ltd), pp. 3156. 
Cavallo, V., Berthelon, C., Mestre, D., and Pottier, A., 1995, Visual information and perceptual style in time-to-collision estimation, In A.G. Gale., I.D. Brown., C.M. Haslegrave and S.P. Taylor (eds) Vision in Vehicles VI (Amsterdam Netherlands: Elsevier Science Publisher B.V.), pp. 81- 89.

Cavallo, V., Laya, O., Laurent, M., 1986, The estimation of time-to-collision as a function of visual stimulation, In A.G. Gale, M.H., Freeman, C.M. Haslegrave, P. Smith, and S.P. Taylor (eds) Vision in Vehicles (Amsterdam Netherlands: Elsevier Science Publisher B.V.). pp. 179-183.

Champion, A., Mandiau, R., Kolski, C., Heidet, A., and Kemeny, A., 1999, Traffic generation with SCANeR II Simulator: towards a multi-agent architecture, In Proceedings of Driving Simulation Conference (DSC'99), Paris, France, pp. 311-324, July 7-8, 1999.

Charles, O., and Espié, S., 2000, Multi-level environments modelling for road simulation, In Proceedings of Driving Simulation Conference (DSC'00), Paris, France, pp. $99-113$, September 6-7, 2000.

Chatterjee, K., McDonald, M., Paulley, N., and Taylor, N. B., 1999, Modelling the impacts of telematics: current limitations and future Developments. Transport Reviews. 19 (1): 57-80.

Chrstos, J. P., and Heydinger, G. J., 1997, Evaluation of VDANL and VDM RoAD for predicting the vehicle dynamics of a 1994 Ford Taurus, In Research into Vehicle Dynamics and Simulation, SAE Paper 970566, Warrendale, PA, pp. 119 - 133.

Churchland, P., Ramachandran, V. C., and Sejnowski, P., 1994, A critique of pure vision, In C. Koch and J. Davis, (eds) Large-Scale Neuronal Theories of the Brain (Cambridge, MA: MIT Press), pp. 23-60. 
Cliff, D., Husbands, P., and Harvey, I., 1993, Evolving visually guided robots, In J.A. Meyer, H. Roitblat and S. Wilson (eds.) From Animals to Animats 2: Proceedings, of the Second International Conference on Simulation of Adaptive Behaviour (Cambridge MA: MIT Press Bradford Books), pp. 374--383.

Colbourn, C., Brown, I. D., and Copeman, A. K., 1978, Drivers' judgements of safe distances in vehicle following. Human Factors. 20 (1): 1-11.

Cremer, J and Kearney, J 1994, Scenario control for virtual environments, In Proceedings of the IMAGE VII Conference, Tucson, AZ, pp. 141-149, June 12-17, 1994.

Cremer, J., Kearney, J., and Willemsen, J., 1996, A directable vehicle behaviour model for virtual driving environments, In Proceedings of the1996 Conference of AI, Simulation, and Planning Autonomy Systems, La Jolla CA, pp. 18-25, March 23-27 1996.

Cremer, J., Kearney, J., Papelis, Y., and Romano, R, 1994, The software architecture for scenario control in the Iowa driving simulator, In Proceedings of the fourth Computer Generated Forces and Behavioural Representation Conference, Orlando, FL, pp. 1-9, May 4-6, 1994.

Crowley, J. L., 1987, Coordination of action and perception in a surveillance robot. IEEE Expert, 2 (4): 32-43.

Dia, H., and Purchase, H., 1999, Modelling the impacts of advanced traveller information systems using intelligent agents. Road and Transport Research Journal, 8 (3): 68-73.

Drew, D.R., 1968, Traffic flow theory and control. (New York: McGraw-Hill).

Druitt, S., 1998, An Introduction to microsimulation. Traffic Engineering \& Control, 39 (11): 480-483. 
Dumbuya, A.D., and Wood, R.L., (in press), Visual perception modelling for virtual driver agents in a synthetic driving simulator, In A.G. Gale et al. (eds.), Vision in Vehicles IX (Amsterdam Netherlands: Elsevier Science Publisher B.V.).

Dumbuya, A.D. 1999, Artificial vision for autonomous software agents. Internal MPhil. Transfer Report, Department of Manufacturing Engineering, Loughborough University, Loughborough, September 1999.

Dumbuya, A.D., and Wood, R.L. 2001, Measuring drivers ability to estimate distance and speed, Association of Industrial Road Safety Officers Meeting, Hertfordshire, UK, pp. 1-5, March 21, 2001.

Dumbuya, A.D., Wood, R.L., and Thomas, P., 2002a, A computational model of visual information processing: mechanisms underlying intelligent driver behaviour, In $33^{\text {rd }}$ European Conference on Mathematical Psychology, Bremen, Germany, pp.20, August 21-24, 2002.

Dumbuya, A.D., Wood, R.L., Gordon, T.J., and Thomas, P., 2002b, An agent-based traffic simulation framework to model intelligent virtual driver behaviour, In Proceedings, Driving Simulation Conference (DSC'02), Paris, France, pp.363-373, September 11-13, 2002.

Dumbuya, A. D., and Wood, R. L., 2002c, A computational model of visual information processing: mechanisms underlying intelligent driver behaviour, Talk given at the Multisensory Research Group Meeting, University Laboratory of Physiology, University of Oxford, UK, February 26, 2002.

Dumbuya, A. D., and Wood, R.L., 2003, Visual perception modelling for intelligent virtual driver agents in synthetic driving simulation. Journal of Experimental and Theoretical Artificial Intelligence (JETAI), 15 (1): 73-102.

Erol, K., Levy, R., and Wentworth, J., 1998, Application of agent technology to traffic simulation, In Proceedings, Complex Systems, Intelligent Systems, and Interfaces, Nimes, France, pp. 1-6, May 26-27, 1998. 
Fernandes, J. M., and Oliveira, E., 1999, TraMas: traffic control through behaviourbased multi-agent system, In Proceedings of the Fourth International Conference on The Practical Applications of Intelligent Agents and Multi-Agent Technology (PAAM99), London, UK, pp. 457-458, April 19-21, 1999.

Franklin, S., and Graesser, A. C., 1997, Is it an agent, or just a program?: a taxonomy for autonomous agents, In J. Mueller, M., Wooldridge, and N., Jennings, (eds) Intelligent Agents III (Berlin:Springer Verlag), pp. 21-35.

Fuller, R. G. C., 1981, Determinants of time headway adopted by truck drivers. Ergonomics, 24 (6): 463-474.

Funge, J. D., 1999, AI for games and animation: a cognitive modelling approach (Natick Massachusetts: A. K. Peters), pp 29-39.

Gibson, J. J., 1950, The perception of the visual world. (New York: Houghton Mifflin), pp 12-43.

Gibson, J. J., 1966, The senses considered as perceptual systems. (New York: Houghton Mifflin), pp 47-184.

Gibson, J. J., 1979, The ecological approach to visual perception. (New York: Houghton Mifflin), pp 147-202.

Gordon, T. J., Best, M.C., and Dixon, P.J. 2002, An automated driver based on convergent vector fields, In Proceedings of the Institution of Mechanical Engineers: Part D, Automobile Engineering. 216, D4, 329-347.

Grand, S., Cliff, D., and Malhotra, A., 1997, Creatures: artificial life autonomous software agents for home entertainment, In M. Del Rey (ed) Autonomous Agents, (California: ACM), pp 22-29. 
Groeger, J. A. and Cavallo, V., 1991, Judgments of time-to-collision and time-tocollision coincidence, In A.G. Gale, I.D. Brown, C.M. Haslegrave, I. Moorhead and S.P. Taylor (eds.) Vision in Vehicles III (Amsterdam Netherlands: Elsevier Science Publisher B.V.), pp. 27-34.

Groeger, J. A., Carsten, O. M. J., Blana, E., and Jamson, H., 1999, Speed and distance estimation under simulated conditions, In A.G. Gale, I.D. Brown., C.M. Haslegrave and S.P. Taylor (eds.) Vision in Vehicles VII (Amsterdam Netherlands: Elsevier Science Publisher B.V.), pp. 291- 299.

Haglund, M., and Åberg, L., 2000, Speed choice in relation to speed limit and influences from other drivers. Transportation Research Part F: Traffic Psychology and Behaviour, 3F (1): 39-51.

Hall, F.L., 1997, Traffic stream characteristics. Traffic Flow Theory: A State-of-theArt Report, Transport Research Board Special Report 165, (Virginia: TurnerFairbank Highway Research Centre), pp 2-36.

Hayes-Roth, B., 1995, An architecture for adaptive intelligent system, Artificial Intelligence: Special Issues on Agents and Interactivity, 72: 329-365.

Hill, F. S., 1990, Computer graphics, (New York: Macmillan Publishing Company), pp. 642-660.

Hill, J., Byard, N., and Thomas, P., 2001, On the spot accident research in the UK: a new approach to in-depth investigations, In Proceedings, Traffic Safety on Three Continents Part 3, Moscow, pp. $96-107$, September 20-22, 2001.

Hoffman, E. R., and Mortimer, R. G., 1994, Drivers' estimates of time to collision. Accident Analysis \& Prevention, 26 (4): 511-520.

Horridge, G. A., 1975, The compound eye and vision of insects. In G.A. Horridge (ed) International Congress of Entomology (Oxford: Clarendon Press), pp. 115-116. 
Hubel, D, H., and Wiesel, T. N., 1962, Receptive fields, binocular interaction and functional architecture in the cat's visual cortex. Journal of Physiology, 160: pp. 106154.

Jain, R., Kasturi, R., and Schunck B. G., 1995, Binary image processing, In Machine Vision (New York: McGraw-Hill, Inc.), pp. 25-70.

Kidd, E.A., and Laughery, K.R., 1964, A computer model of driver behaviour: The highway intersection situation, Cornell Aeronautical Laboratories, Report, no. VJ1843-V-1, Buffalo, New York, June 1964.

Land, M. F., 2000, Does steering a car involve perception of the velocity flow field? In J. M., Zanker and J., Zeil (eds) Motion Vision: Computational, Neural and Ecological Constraints (Berlin: Springer-Verlag), pp. 227-235.

Langer, D., Rosenblatt, J. K., and Hebert, M., 1994, A reactive system for off-road navigation. In Proceedings of IEEE Conference on Robotics and Automation, San Diego, CA, pp. 1-29, May 8-13, 1994.

Langton, C. G., 1989, Artificial life. In C. G. Langton, (ed) Artificial Life VI, SFI Studies in the Sciences of Complexity, (Redwood City, CA: Addison-Wesley), pp. 147.

Lee, D. N., 1976, A theory of visual control of braking based on information about time-to-collision. Perception, 5: 437-459.

Lehmuskoski, V., and Niittymäki, L. T. J., 1999, Development of microscopic simulation model on ring road III near Helsinki-Vantaa international airport, In Proceedings of Urban Transport Systems Conference, Sweden Lund, pp. 1-13, June 7-8, 1999.

Leibowitz, H. W., Guzy, L. T., Peterson, E., and Blake, P. T., 1993, Quantitative perceptual estimates: verbal versus nonverbal retrieval techniques. Perception, 22: 1051-1060. 
Lieberman, E., and Rathi, A.K, 1997, Traffic simulation, In Traffic Flow Theory: $A$ State-of-the-Art Report, Transport Research Board Special Report 165, (Virginia: Turner-Fairbank Highway Research Centre), pp 10-23.

Ludmamn, J., Neunzig, D., and Weilkes, M., 1997, Traffic simulation with consideration of driver models, theory and examples, In P. Lugner and H. K. Karl (eds) Vehicle System Dynamics, 27: pp. 491-516.

Lynch, N., and Wood, A., 1992, The driving manual, Driving Standard Agency, (London: HMSO).

Maher, M., 1998, Stochastic assignment, elastic demand and matrix estimation. In Seminar on Modelling Traffic Networks: Current Research and Future Directions, University of Leeds, U.K, pp 1-4, November 26, 1998.

Manikonda, V., Teittinen, A., and Lovell, D., 2000, Decentralised adaptive agents for control of traffic signals, In Proceedings, Intelligent Systems and Application (ISA'2000), Wollongong, Australia, pp. 1-7, December 12-15, 2000.

Marr, D., 1982, Vision: A Computational Investigation into the Human Representation and Processing of Visual Information, (San Francisco CA: W.H. Freeman and Company), pp 19-34; 99-215.

Masroor, H., Cuneo, D., and Chachich, A., 1997, Analysis of traffic video to develop driver behaviour models for microscopic traffic simulation, In Proceeding of IEEE Conference on Intelligent Transportation Systems, Boston, Massachusetts, pp 747753, November 9-12, 1997.

Mather, G., 2001, Visual physiology

http://www.biols.susx.ac.uk/home/George_Mather/Linked\%20Pages/Physiol/index.ht $m l$ (15 December 2002). 
Maurer, P., 1999, The use of a video traffic analysis system to collect traffic data and the visualisation of the traffic flow with a microscopic simulation model, In Proceeding of $8^{\text {th }}$ International Symposium on Electronics in Traffic ISEP'99, Ljubljana, pp 1-6, October 7-8, 1999.

McDonald, M., Wu, J., and Brackstone, M., 1997, Development of a fuzzy logic based microscopic motorway simulation model, In Proceedings of IEEE Conference on Intelligent Transportation Systems (ITSC'97) ITSC'97, Boston, USA, pp 1-6 November 9-12, 1997.

McLeod, R. W., and Ross, H. E., 1983 Optic-flow and cognitive factors in time-tocollision estimates. Perception, 12: 417-423.

McRuer, D.T., Allen, R.W., Weir, D.H., and Klein, R.H., 1977, New results in driver steering control models. Human Factors, 19 (4): 381-397.

Michon, J. A., 1985, A critical view of driver behaviour models: what do we know, what should we do? In L. Evans and R. Schwing (eds) Human Behavior and Traffic Safety (London: Plenum Press), pp. 485-524.

Moray, N., 1986, Monitoring behaviour and supervisory control. In K. Boff, L. Kaufmann and J. Beatty, (eds.) Handbook of Perception and Human Performance (New York: John Wiley and Sons), pp. 33-42.

Musse, S. R., and Thalmann 1997, A model of human crowd behavior: group interrelation and collision analysis, In Proceeding of Computer Animation and Simulation (CAS'97), EUROGRAPHICS Workshop, Budapest, Hungary, pp. 39-51, September 2-3, 1997.

Neubert, L., Lee, H. Y., and Schreckenberg, M., 1999, Density waves and jamming transition in cellular automata models for traffic flow. J. of Phys. A: Math. Gen. 32: 6517-6525. 
Newell, A., and Simon, H.A., 1972, Human problem solving, Englewood Cliffs, (New Jersey: Prentice Hall), pp 87-150.

Niehaus, A. and Stengel, R. F., 1994, Probability-based decision making for automated highway driving. IEEE Transactions on Vehicular Technology, 43 (3): 626-634.

Noser, H., Renault, O., Thalmann, D., Thalmann, N. M., 1995, Navigation for digital actors based on synthetic vision, memory and learning. Computers and Graphics, 19 (1): 7-19.

Object Management Group: http://www.omg.org/ $\left(10^{\text {th }}\right.$ June 2002).

Ohta, H., 1993, Individual differences in driving distance headway, In A.G. Gale, M.H. Freeman, C.M. Haslegrave, P. Smith and S.P Taylor (eds), Vision in Vehicles (Amsterdam Netherlands: Elsevier Science Publisher B.V.), pp. 91-100.

Paniati, J. F., and True, J., 1996, Interactive Highway Safety Design Model (IHSDM): designing highways with safety in mind, In Transportation Research Circular No. 453, National Research Council, Washington, D.C., pp. 55-60.

Papelis, Y. E., and Bahauddin, S., 1995, Logical modelling of roadway environment to support real-time simulation of autonomous traffic. In Proceedings of the First Workshop on Simulation and Interaction in Virtual Environments (SIVE95), (University of Iowa), Iowa U.S.A, pp. 62-71, July 13-15, 1995.

Papelis, Y., 1996, Graphical authoring of complex scenarios using high level coordinators, In Workshop on Scenario and Traffic Generation in Driving Simulation, Orlando, USA, pp. 3-10, December 6-7, 1996.

ParallelGraphics: http://www.parallelgraphics.com/ (15 May 2002) 
Pierzchala, M., and Rodanski, B., 1993, A new method of semi-symbolic network analysis, IEEE International Symposium on Circuits and Systems, ISCAS, Chicago, Illinois, pp. 2240-2243, May 3-6, 1993.

Plastock, R. A., and Kalley, G., 1986, Schaum's outline series theory and problems of computer graphics (New York: McGraw-Hill Cook Company), pp. 1-309

Pomerleau, D. A., 1993, Neural network perception for mobile robot guidance. (Boston: Kluwer Academic Publishers), pp. 1-159

Pursula, M., 1999, Simulation of traffic systems - an overview. Journal of Geographical Information and Decision Analysis, 3 (1): 1-8

Rabie, T. F., and Terzopoulos, D., 2000, Active perception in virtual humans, In Proceedings of the $13^{\text {th }}$ Canadian Vision Interface Conference (VI 2000), Montreal, Quebec, pp 1-7, May 14-17, 2000.

Recarte, M. A., and Nunes, L. M, 1998, Effects of distance and speed on time to arrival in an automobile: two classes of time? In A.G. Gale., I.D. Brown, C.M. Haslegrave and S.P. Taylor (eds.), Vision in Vehicles VI (Amsterdam Netherlands: Elsevier Science Publisher B.V.). pp. 63-71.

Renault, O., Magnenat-Thalmann, N., Thalmann, D., 1990, A vision-based approach to behavioural animation. The Journal of Visualisation and Computer Animation, 1: 18-21

Rensink, R., and Boer, E., 1996. The big picture. In Cambridge Basic Research Annual Report, pp. 1-9

Reynolds, C. W., 1987, Flocks, herds and schools: A distributed behavioral model. Computer Graphics, 21(4): 25-34

Richard, C., 1999, Human decision making, Recovery, A Quarterly Journal on Roadway Crashes, Injuries, and Healing, 10 (4): 1-4. 
Rothery, R. W., 1997, Car following models, In Traffic Flow Theory, Transportation Research Board Special Report 165, (Virginia: Turner-Fairbank Highway Research Centre), pp 4-42.

Russell, R. A., 1990, Robot tactile sensing (London: Prentice Hall).

Sabeur, M., and Rommelfanger, R., 2000, A new software tool for generating Roads networks for driving simulation, In Proceedings of the Driving Simulation Conference (DSC' 00), Paris, France, pp. 149-162, September 6-8, 2000.

Salgian, G., and Ballard, D., H., 1998a, Visual routines for vehicle control, In D. Kriegman, G. Hager, S. Morse (eds), The Confluence of Vision and Control (Berlin: Springer Verlag), pp 1-13.

Salgian, G., and Ballard, D., H., 1998b, Visual routines for autonomous driving, In Proceedings of the $6^{\text {th }}$ International Conference on Computer Vision (ICCV), Bombay, India, pp. 876-882, January 4-7, 1998.

Salomon, R., 1996, Neural networks in the context of autonomous agents: important concepts revisited, In Proceedings of the Artificial Neural Networks In Engineering (ANNIE'96), New York, USA, pp. 109-116, November 10-13, 1996.

Santos, J. A., 1998, Vehicle-motion detection: inhibitory effects of self-motion and optical flow structure, In A.G. Gale., I.D. Brown, C.M. Haslegrave and S.P. Taylor (eds.), Vision in Vehicles VI (Amsterdam Netherlands: Elsevier Science Publisher B.V.). pp. 289-295.

Sayers, M.W., 1998, Vehicle models for RTS applications, In Proceeding of the International Symposium on Advanced Vehicle Control, AVEC'98, Nagoya, Japan, pp 1-6, September 14-18, 1998.

Schiff, W., and Detwiler, M. L., 1979, Information used in judging impending collision. Perception, 9: 647-658. 
Seif El-Nasr, M and Skubic, M, 1998, A fuzzy emotional agent for decision making in mobile robot. In Proceedings of 1998 International Conference On Fuzzy Systems (FUZZ-IEEE'1998), Anchorage, Alaska, pp.135-140, May 4-9, 1998.

Shoham, Y., 1993, Agent-oriented programming. Artificial Intelligence, 60 (1): pp. $51-92$.

Smith, M. J., Xiang, Y., Yarrow, R.A., and Ghali, M., 1996, Bilevel and other modelling approaches to urban traffic management and control, In Proceedings, Equilibrium and Advanced Transportation Modelling, Montreal, Canada, Spring '96, pp. 283-325.

Stark, L. W., Privitera, C. M., Yang, H., Azzariti, M., Ho, Y. F., Blackmon, T., and Chernyak, C., 2001. Representation of human vision in the Brain: how does human perception recognize images? Journal of Electronic Imaging Special Issue on Human Vision 10 (1): 123-151.

Sukthankaar, R., 1997, Situation awareness in tactical driving, $\mathrm{PhD}$ Thesis, Robotics Institute, Carnegie-Melon University.

Sukthankar, R., 1996, Teaching intelligent vehicles to drive in traffic: tactical-level scenarios, In Workshop on Scenario and Traffic Generation for Driving, (University of Iowa), Iowa City, U.S.A, pp. 1-2, July 13-15, 1995.

Taieb-Maimon, M. and Shinar, 2001, Minimum and comfortable driving headways: Reality versus perception. Human Factors, 43 (1): 159-172.

Taylor, M.A.P., Young, M. and Bonsall, P.W., 1996, Basic traffic flow theory. In Understanding Traffic Systems: Data, Analysis and Presentation (Hants, England: Ashgate Publishing Limited), pp. 37-63.

Terzopoulos, D., and Rabie, T. F., 1997, Animat vision: active vision in artificial animals. Journal of Computer Vision Research, 1 (1): 2-19 
Terzopoulos, D., Rabie, T. F., and Grzeszczuk, R., 1996, Perception and learning in artificial animals, In Proceedings of Fifth International Conference on the Synthesis and Simulation of Living Systems, Nara, Japan, pp. 1-8, May 16-18, 1996.

Thalmann, D., 1995, Virtual sensors: a key tool for the artificial life of virtual actors, In Sung Yong Shin, Tosiyasu, L. Kun (eds) Computer Graphics and Applications (New Jersey: River Edge World Scientific), pp. 22-40.

Thalmann-Magnenat, N., and Thalmann, D., 1994, Introduction: creating artificial life in virtual reality. In M. N. Thalmann and D. Thalmann (eds) Artificial Life and Virtual Reality (John Wiley and Sons Ltd), pp 1-11.

Tran, J. M., 1995, Pedagogical environment for driving simulators: traffic generation and intelligent aids to scenario control, In Proceedings, Driving Simulation Conference (DSC'95), Sophia-Antipolis, France, pp 246-259, September 12-13, 1995.

Tu, X., 1999, Artificial animals for computer animation: biomechanics, locomotion, perception and behaviour. In G. Goos, J. Hartmanis and J. van Leeuwen (eds) Lecture Notes in Computer Science (Berlin: Springer-Verlag), pp. 65-83.

Tu, X., and Terzopoulos, D., 1994, Perceptual modelling for behavioral animation of fishes. In Proceedings Second Pacific Conference on Computer Graphics and Applications, Beijing, China, pp. 185-200, August 26-29, 1994.

van Droogenbroeck, M, 2002, Algorithms for openings of binary and label images with rectangular structuring elements. In H. Talbot and R. Beare, (eds) Mathematical morphology (Sydney Australia: CSIRO Publishing), pp. 197-207.

van Winsum W., and Heino, A., 1996, Choice of time-headway in car-following and the role of time-to-collision information in braking. Ergonomics, 39 (4): 579-592. 
van Wolffelaar, P. C., and van Winsum, W., 1995, Traffic modelling and driving simulator - an integrated approach, In Proceedings of the Driving Simulation Conference (DSC'95), Sophia-Antipolis, France, pp. 236-245, September 12-13, 1995.

van Wolffelaar, P., Bayarri, S., and Coma, I., 1999, Script-based definition of complex scenarios, In Proceedings of Driving Simulation Conference (DSC'99), Paris, France, pp. 353-365, July 7-8, 1999.

VRlab, EPFL, Lausanne, http://vrlab.epfl.ch (22 December 2002)

Wallis, G., Tresilian, J., Chatziastros, A, 2000, The influence of non-visual cues on steering behaviour. Perception, 29: No. ECVP Supplement, p. 121-122.

Wandell, B. A., 1995, Foundations of vision, (Massachusetts: Sinauer Associates, Inc.), pp 1-402.

Weir, D. H., and McRuer, D. T., 1970, Dynamics of driver vehicle steering control, Automatica, 6: 87-98.

Wong, J. Y., 2001, Theory of Ground Vehicles (New York: John Wiley \& Sons, Inc.)

Wood, R. L., and Arnold, J.E. 1997. An automata based simulation of co-operative decision making. In R. A. Adey, G. Rzevski and R. Teti (eds) Applications of Artificial Intelligence in Engineering, (Southampton, UK: Computational Mechanics Publications), pp. 105-109.

Wood, R.L., Dumbuya, A.D., Hill, J. R., Thomas, P. and Zhao, Q., 2003, Simulation of driver, vehicle and environmental aspects of crash initiation - a new method to improve integrated safety effectiveness, Paper Accepted for the 18th International Conference on the Enhanced Safety of Vehicles, (ESV), Nagoya, Japan, May 19-22.

Wooldridge, M., and Jennings, N. R., 1995, Intelligent agents: theory and practice. The Knowledge Engineering Review, 10 (2): 115-152. 
Wright, C., and Roberg-Orenstein, P., 1998, Congestion control: a qualitative approach based on simple traffic jam models. In Seminar on Modelling Traffic Networks: Current Research and Future Directions, University of Leeds, U.K, pp 119, November 26, 1998.

Wright, S., 1998, A framework for supporting intelligent traffic within the Leeds Driving Simulator. In Proceedings, Workshop on Intelligent Virtual Environments, $13^{\text {th }}$ European Conference on Artificial Intelligence (ECAI'98), Brighton, UK, pp 1-6 August 23-28, 1998.

Wright, S., 2000a, DRIVERSIM: A probabilistic approach to realistic traffic Generation, In Proceedings of Driving Simulation Conference (DSC' 00), Paris, France, pp. $267-274$, September 6-8, 2000.

Wright, S., 2000b, Supporting intelligent traffic in the Leeds driving simulator. $\mathrm{PhD}$ thesis, The University of Leeds, UK.

Wu, J., Brackstone, M, and McDonlad, M., 2000, Fuzzy sets and systems for a motorway microscopic simulation model. International Journal of Fuzzy Sets and Systems, $116(1)$ : 65-76.

Wu, J., McDonlad, M., and Brackstone, M., 1998, A fuzzy logic microscopic simulation model for interurban ATT assessment. In Proceedings of $10^{\text {th }}$ European simulation symposium (ESS'98), Nottingham, pp. 347-354, October 26-28, 1998.

Yaeger L., 1994, Computational genetics, physiology, metabolism, neural systems, learning, vision, and behaviour or PolyWorld: Life in a New Context. In C. Langton (ed), Artificial Life III, SFI Studies in the Sciences of Complexity, (Reading: AddisonWesley), pp. 263-297. 


\section{APPENDIX A}

\section{LIST OF PUBLICATIONS}

\section{Journal Papers}

Dumbuya, A. D., and Wood, R. L., 2003, Visual perception modelling for intelligent virtual driver agents in synthetic driving simulation. Journal of Experimental and Theoretical Artificial Intelligence (JETAI), 15 (1): 73-102.

\section{Referred Conference Papers}

Dumbuya, A. D., and Wood, R. L., (in press), Visual perception modelling for virtual driver agents in a synthetic driving Simulator, In A.G. Gale et al. (eds.), Vision in Vehicles IX (Amsterdam Netherlands: Elsevier Science Publisher B.V.).

Dumbuya, A. D., Wood, R. L. and Thomas, P., 2002a, A computational model of visual information processing: mechanisms underlying intelligent driver behaviour. In $33^{\text {rd }}$ European Conference on Mathematical Psychology, International University of Bremen, Bremen, Germany, pp.20.

Dumbuya, A. D., Wood, R. L., Gordon, T. J., and Thomas, P., 2002b, An agent-based traffic simulation framework to model intelligent virtual driver behaviour, In Proceedings, Driving Simulation Conference (DSC'02), Paris, France, pp.363-373.

Wood, R. L., Dumbuya, A. D., Hill, J. R., Thomas, P. and Zhao, Q., 2003, Simulation of driver, vehicle and environmental aspects of crash initiation - a new method to improve integrated safety effectiveness, Paper Accepted for the 18th International Conference on the Enhanced Safety of Vehicles, (ESV), Nagoya, Japan.

\section{Other Conference Papers or Poster Presentations}

Dumbuya, A. D., and Wood, R .L, 2001, Measuring drivers ability to estimate distance and speed, In Association of Industrial Road Safety Officers Meeting, Hertfordshire, March $21^{\text {st }} 2001$. 
Dumbuya, A. D., and Wood, R. L., 2001a, A software model of visual perception as part of emergent behaviour in a synthetic driving simulator, Poster Presentation at the International Conference of Vision In Vehicle IX, Brisbane, Australia.

Dumbuya, A. D., and Wood, R. L., 2002c, A computational model of visual information processing: mechanisms underlying intelligent driver behaviour, Talk given at the Multisensory Research Group Meeting, University Laboratory of Physiology, University of Oxford, UK, February $26^{\text {th }} 2002$.

\section{Internal Reports}

Dumbuya, A. D., 1999, Artificial vision for autonomous software agents, Internal MPhil. Transfer Report, Department of Manufacturing Engineering, Loughborough University, Loughborough, September 1999.

Dumbuya, A. D., and Wood, R. L., 2001b, Framework for realistic traffic simulation, Department of Manufacturing Engineering, Loughborough University. Presentation and Report Prepared for Institute of Consumer Ergonomics (ICE), Loughborough, February 14, 2001. 


\section{APPENDIX B}

\section{SELECTED UNIFIED MODELLING LANGUAGE (UML) DIAGRAMS}

B.1 Attributes and Methods of Key Classes of $\underline{S} y n t h e t i c$ Driving $\underline{\text { Simulation (SD-SIM) }}$

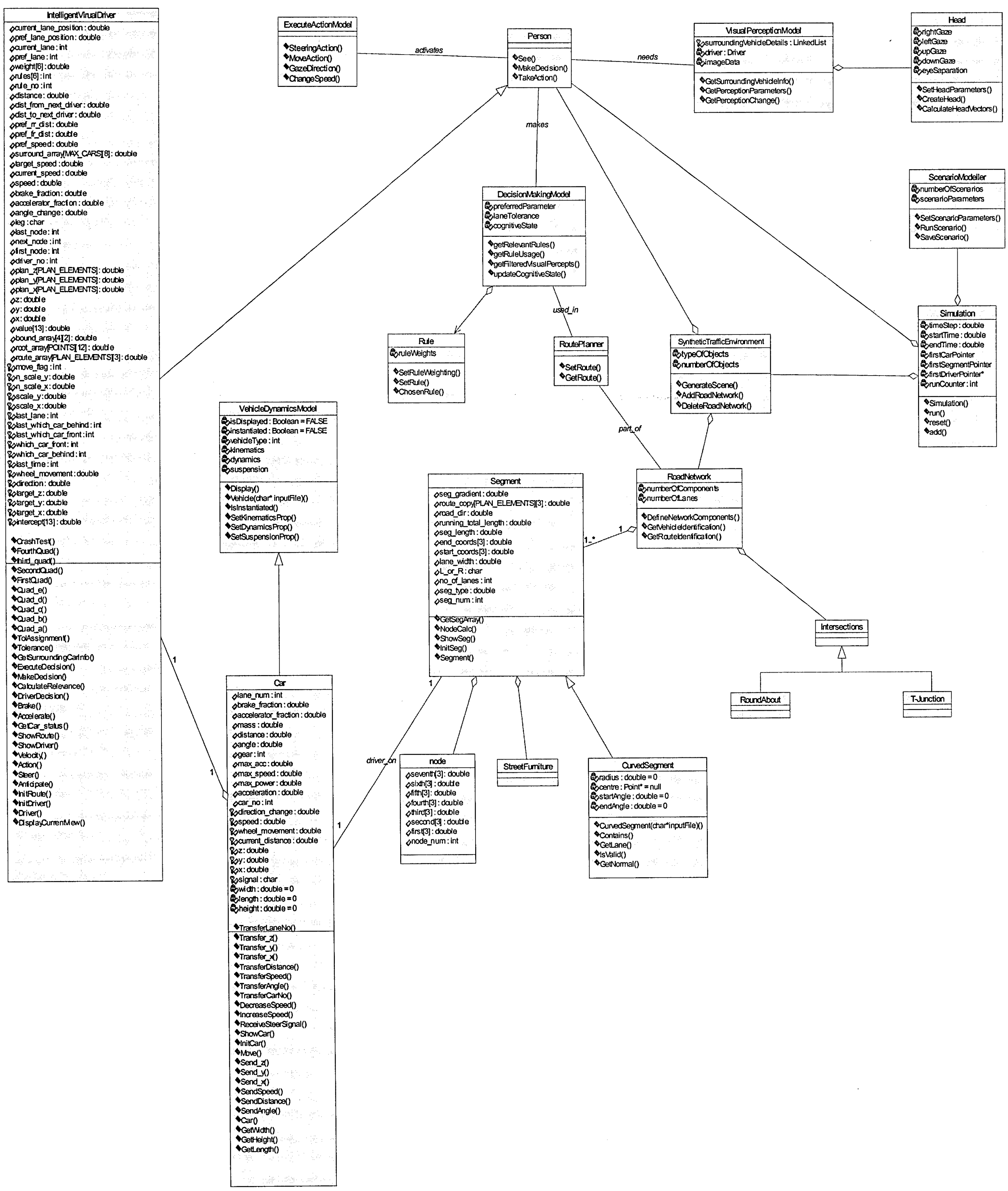




\section{Notes on Appendix B.1}

Appendix B.1 provides a detailed diagram showing the attributes and methods used in SD-SIM. Explanation of the main classes and attributes of this diagram is given in chapter 4, section 4.5.1. The implementation of SD-SIM based on instances of these classes supports the creation and manipulation of traffic simulation entities.

Driver and Vehicle classes used in figure 4.15 in section 4.5 .1 are the same as IntelligentVirtualDriver and VehicleDynamicsModel used in appendix B.1. The difference in naming convention is simply to reflect the detailed conveyed in appendix B.1. The other classes that were not discussed in section 4.5 .1 are Head, CurvedSegment. Head in appendix B.1 diagram is part of the VisualPerceptionModel class which contain the attributes and methods used to create an instance of a Head and calculate the necessary head parameters. A more detailed diagram of the Head class is given appendix B.2. A CurvedSegment is inherited from Segment class. The functionality of a CurvedSegment has not been implemented because of the current minimal functionality of the synthetic traffic environment. 


\section{B.2 Attributes and Methods of Key Classes of Scene En-capturing and Evaluation (SEE)}

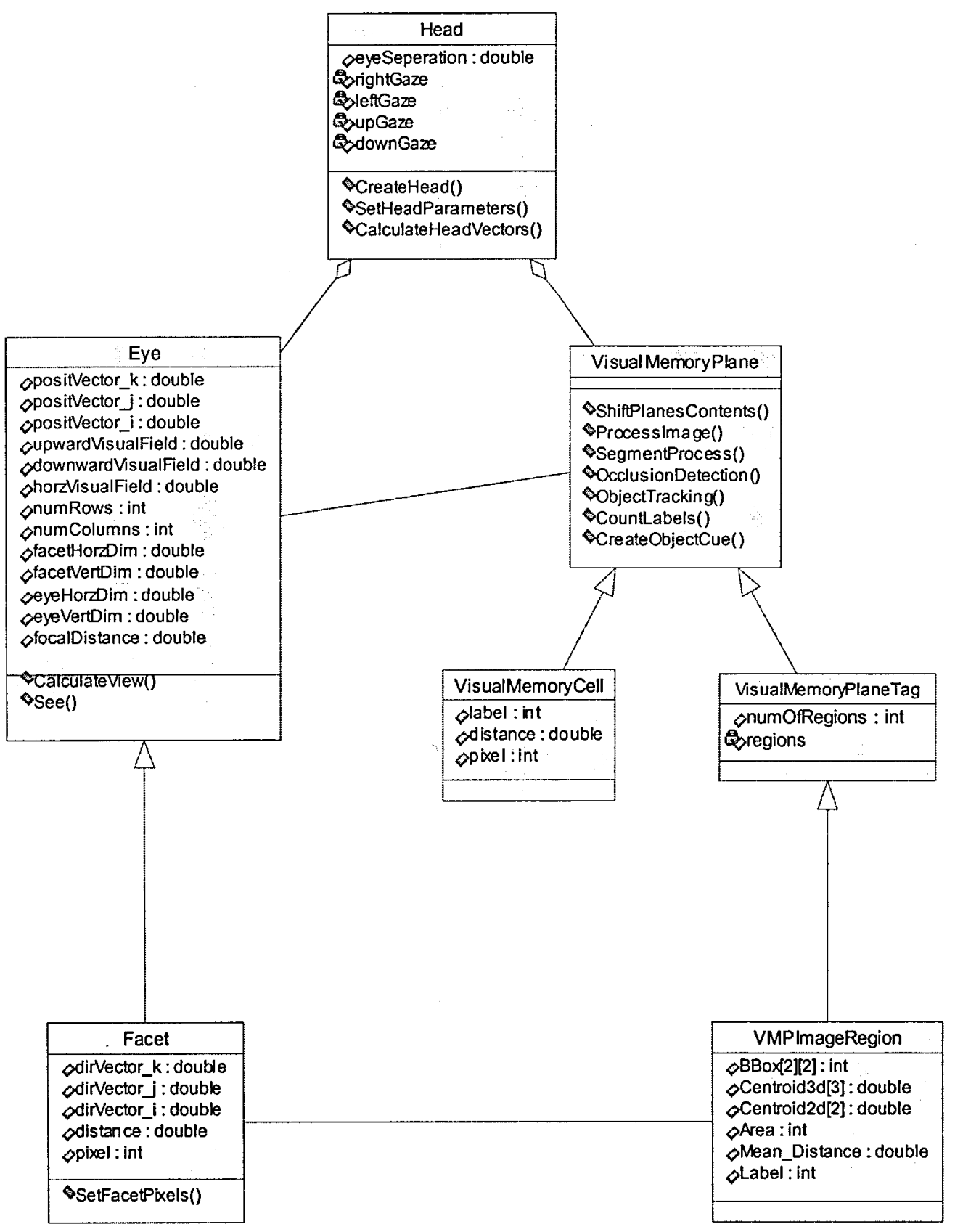

\section{Notes on Appendix B.2}

Appendix B.2 summarises the structure of SEE. A thorough description of SEE is given in sections 4.4.1, 4.4.2 and 4.4.3. In the diagram of appendix B.2, Head is a super class and is made up of Eye and VisualMemoryPlane. Eye contain a number of 
variables such as the position vectors of the eyes, the field of view defined for each eye, the number of columns, focal distance and eye dimensions. The eyes performed 'seeing' function. A Facet is inherited from each eye which calls on a method to set the pixel of that facet. Eye has a simple relationship with VisualMemoryPlane. The VisualMemoryPlane contain several methods to process the images registered on the eyes. Each memory plane has a VisualMemoryCell and VisualMemoryPlaneTag. Each VisualMemoryPlaneTag has an inheritance association with VMPImageRegion which is a class that is responsible for calculating bounding box properties, the centroids of $2 D$ image regions and $3 D$ object centroids. 


\section{APPENDIX C}

\section{DATA COLLECTION TOOLS}

\section{C.1 A Questionnaire to Asses Subjects Driving Behaviour}

The design of the questionnaire was intended to solicit relevant information pertaining to the subjects' gender, age, driving experience, profession etc. The author felt this information was relevant to the research activity based on initially identifying those concepts or variables that needed to be measured. Although the questionnaire instrument provided a structured way of obtaining this information, it's designed and development was time consuming, mainly due to the choice of questions. Below were the questions the subjects had to answer prior to the commencement of the experiments.

(All details given in this questionnaire will be kept in strictest confidence)

Subject Number:

1. Name:

2. Contact details:

Address:

Day Telephone Number:

3. How would you describe your vision? Good

Poor Average

4. Do you need glasses to drive? Yes No

5. $\operatorname{Sex} \mathrm{M} \square$<smiles>F[C]1CCC1</smiles>

6. Age: 
7. Please state your height:

8. a. How long have you been driving?

b. How frequently do you drive

i. How many miles a year?

ii. How many times a week?

iii. How long are your typical journey?

9. Please tick if you have a clean driving license: $\square$

10. Please state you profession

\section{C.2 General Instructions}

The general instructions tool was used to provide the subjects with a clear and brief description of the experiments in terms of the purpose of the experiments, how the experiments will be conducted and a reminder of the safety precaution that will be taken. In addition, this activity was also intended to solicit the subjects' consent, with the option of opting out of the experiments. The comments below were explained to each subject and only when they had understood the nature of the experiments, did they signed on the table, shown below.

These experiments are aimed at collecting data to validate a computer simulation of driving behaviour.

You will be asked a series of questions or to perform some tasks in each experiment. The experiments are conducted in a control environment and we believe that you will not be placed at any risk during these experiments. However, please feel free to ignore any instructions if you feel you are at risk. Also you may withdraw from these experiments at any stage without giving a reason. If you understand the nature of the experiments and would like to participate please sign below. 


\begin{tabular}{|c|l|l|c|l|l|}
\hline $\begin{array}{l}\text { Subject } \\
\text { Number }\end{array}$ & YES/NO & Signature & $\begin{array}{l}\text { Subject } \\
\text { Number }\end{array}$ & YES/NO & Signature \\
\hline 1 & & & 19 & & \\
\hline 2 & & & 20 & & \\
\hline 3 & & & 21 & & \\
\hline 4 & & & 22 & & \\
\hline 5 & & & 23 & & \\
\hline 6 & & & 24 & & \\
\hline 7 & & & 25 & & \\
\hline 8 & & & 26 & & \\
\hline 9 & & & 27 & & \\
\hline 10 & & & 28 & & \\
\hline 10 & & & 29 & & \\
\hline 11 & & & 30 & & \\
\hline 12 & & & 31 & & \\
\hline 13 & & & 32 & & \\
\hline 14 & & & 33 & & \\
\hline 15 & & & 34 & & \\
\hline 16 & & & 35 & & \\
\hline 17 & & & 36 & & \\
\hline 18 & & & 37 & & \\
\hline
\end{tabular}

\section{C.3 Graphical Rating Scales}

A graphical rating scale has been used to assess subjects' performance in the estimation of distance and speeds. The choice of the rating scale tool for these experiments was mainly due to the fact that humans are very poor at quantifying distances and speeds. Therefore the scales will help remove possible errors that individuals make in converting their perception to numerical values at the time of perception. Leibowitz et al (1993) provides a thorough discussion of the problems associated with verbalisation techniques, including the accuracy of verbal and nonverbal methods.

The rating scales in figure C.3a were used in experiments 2 (stop and go experiment) and 3 (dynamic situation). The scales were used to assess subjects' estimation of distances and speeds of the approaching target vehicle at each measured distance and speed and at each time step in experiment 1 (see 6.3.1.1 for detailed description of the 
procedures). The range of distance positions of the approaching vehicle was classified into 'Near' and 'Far'. If the subject perceived the vehicle to be far, they will indicate this closer to the 'Far' point of the scale and simultaneously add on the speed scale whether the speed to this point was 'Fast' or 'Slow'. As the vehicle approached closer, it is expected that there will be a cluster of points closer to 'Near'. As well as marking on the scale their sense of 'Near' or 'Far' and 'Slow' or 'Fast', the subjects also added a value corresponding to this mark. The comparison of the subjects' non-numerical and numerical perception provided a qualitative assessment of their meaning of these variables.

The procedure for the dynamic speed experiment (experiment 3) was similar to experiment 2 (see section 6.3.1.1 for more detailed description of the procedure). However, for experiment 3 , the subject was only provided with the speed scale. All the scales with the subjects' perception were processed.

Please indicate by marking on the scale and adding a value (given in any units) of what your estimate of the position (distance) to the target vehicle at this time period was.

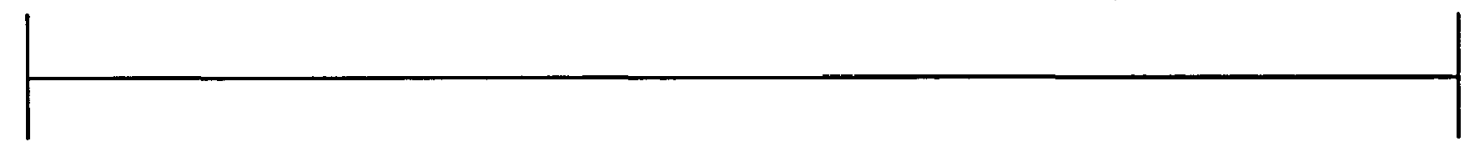

Near

Please indicate by marking on the scale and adding a value (given in any units) of what your estimate of the speed of the target vehicle at this time period was.

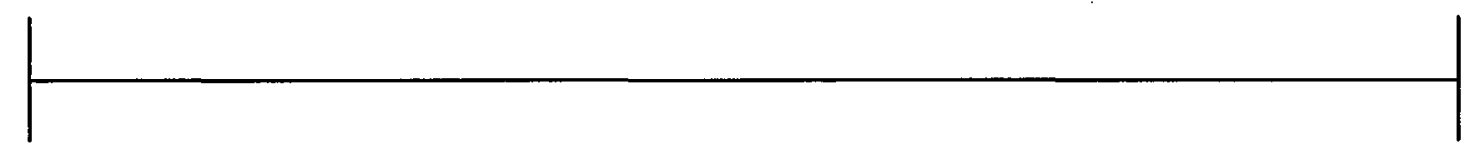

Slow

Figure C.3a: Rating scales for experiment 2 and 3. 
